Neutron and Charged-Particle Induced Cross Sections for Radiochemistry for Isotopes of Scandium, Titanium, Vanadium, Chromium, Manganese, and Iron

K. Kelley, R. D. Hoffman, F. S. Dietrich, R. Bauer, M. Mustafa

April 25, 2005 
This document was prepared as an account of work sponsored by an agency of the United States Government. Neither the United States Government nor the University of California nor any of their employees, makes any warranty, express or implied, or assumes any legal liability or responsibility for the accuracy, completeness, or usefulness of any information, apparatus, product, or process disclosed, or represents that its use would not infringe privately owned rights. Reference herein to any specific commercial product, process, or service by trade name, trademark, manufacturer, or otherwise, does not necessarily constitute or imply its endorsement, recommendation, or favoring by the United States Government or the University of California. The views and opinions of authors expressed herein do not necessarily state or reflect those of the United States Government or the University of California, and shall not be used for advertising or product endorsement purposes.

This work was performed under the auspices of the U.S. Department of Energy by University of California, Lawrence Livermore National Laboratory under Contract W-7405-Eng-48. 
UCRL-TR-211668

\title{
Neutron and Charged-Particle Induced Cross Sections for Radiochemistry for Isotopes of Scandium, Titanium, Vanadium, Chromium, Manganese, and Iron
}

\author{
K. Kelley, R. D. Hoffman, F. S. Dietrich and R. Bauer \\ Nuclear Theory and Modeling Group \\ Physics and Advanced Technologies, N-Division \\ Lawrence Livermore National Laboratory \\ Livermore, CA 94550 \\ kelley24@llnl.gov \\ M. Mustafa \\ Nuclear and Defense Technologies, AX-Division \\ Lawrence Livermore National Laboratory \\ Livermore, CA 94550
}

\begin{abstract}
We have developed a set of modeled nuclear reaction cross sections for use in radiochemical diagnostics. Local systematics for the input parameters required by the Hauser-Feshbach statistical model were developed and used to calculate neutron, proton, and deuteron induced nuclear reaction cross sections in the mass region of scandium, titanium, vanadium, chromium, manganese, and iron $(21 \leq \mathrm{Z} \leq 26,20 \leq \mathrm{N} \leq 32)$.
\end{abstract}

Subject headings: Nuclear cross sections, Radiochemistry, Nuclear Physics

\section{Introduction}

\subsection{Radiochemistry}

Various aspects of nuclear explosive device performance can be determined through the use of radiochemistry. During the UGT (Under Ground Test) Program, select naturally occurring elements were loaded into a device prior to a test and their activation products subsequently retrieved for counting, typically with gamma-ray detectors. The products are measured as isotopic ratios (such as ${ }^{87} \mathrm{Y} /{ }^{88} \mathrm{Y}$ produced from the stable isotope of the naturally occurring element). From the measured activity and prior knowledge of the amount of loaded detector material, performance aspects could be inferred by comparing the measured iso-

\footnotetext{
${ }^{1}$ Department of Physics, University of California, Davis Davis, CA 95616
}

tope ratios with those calculated using neutron and charged-particle fluences from one of the design codes and group-averaged cross section sets prepared for this purpose.

This paper develops new neutron and charged particle detector sets for loaded detector elements titanium, chromium, and iron. We restrict our discussion to unclassified data related to the modeling effort. Previous efforts have performed a similar analysis for three other RADCHEM charged particle detector sets, ${ }^{79} \mathrm{Br}$ producing ${ }^{79} \mathrm{Kr}$ (Hoffman et al. 2004), ${ }^{127} \mathrm{I}$ producing ${ }^{127} \mathrm{Xe}$ (Hoffman et al. 2004), and stable Europium producing ${ }^{147-150,152,154} \mathrm{Eu}$ and ${ }^{151,153} \mathrm{Gd}$ (Hoffman et al. 2004). A separate classified document will discuss Stockpile Stewardship applications. 


\section{Contents}

1 Introduction $\quad 1$

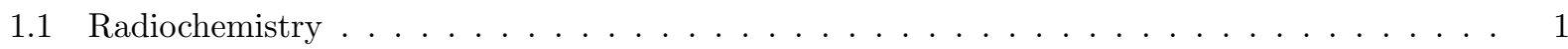

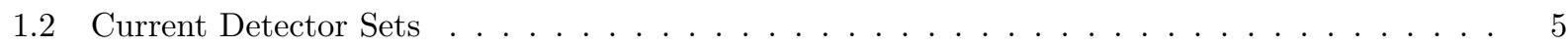

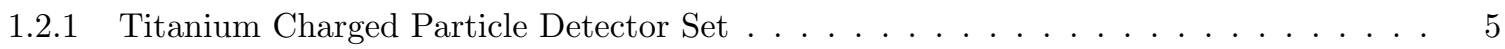

1.2.2 Chromium and Iron Charged Particle Detector Sets . . . . . . . . . . . . . . . . 5

1.3 The Need for New Detector Sets $\ldots \ldots \ldots \ldots \ldots \ldots$

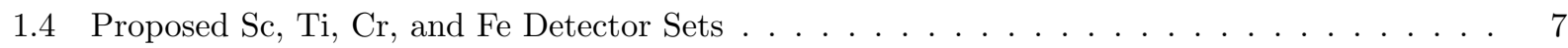

2 Nuclear Reaction Theory $\quad 7$

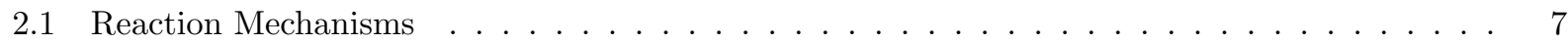

2.2 Hauser-Feshbach Statistical Model . . . . . . . . . . . . . . . . . . 7

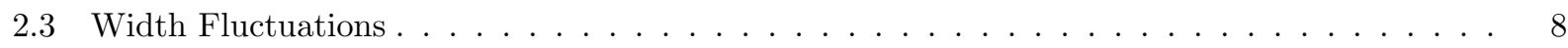

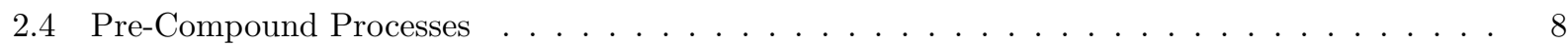

2.5 The STAPRE Hauser-Feshbach Reaction Code $\ldots \ldots \ldots \ldots \ldots \ldots$

3 Inputs Required for the Hauser-Feshbach Model $\quad 9$

3.1 Nuclear Structure Data . . . . . . . . . . . . . . . . . . . . . . . . . . . . 9

3.1 .1 Nuclear Masses and $J^{\pi}$ Assignments . . . . . . . . . . . . . . . . . . . . 9

3.1 .2 Nuclear Level Schemes . . . . . . . . . . . . . . . . . . . . . . . . . 9

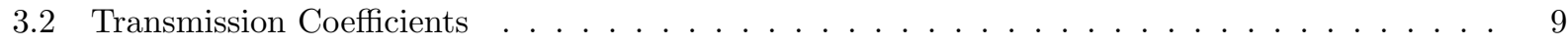

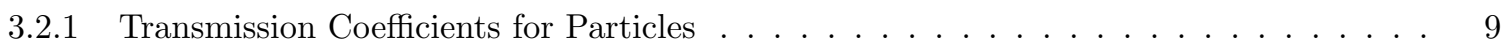

3.2 .2 The Optical Potential of Koning and Delaroche . . . . . . . . . . . . . . . . . . 9

3.2.3 Evaluation of the Neutron and Proton Optical Potential . . . . . . . . . . . . . . . 10

3.2 .4 The Alpha and Deuteron Optical Potentials . . . . . . . . . . . . . . . . . . 12

3.2.5 Transmission Coefficients for Photons . . . . . . . . . . . . . . . . . . 12

3.3 Nuclear Level Densities . . . . . . . . . . . . . . . . . . . . . . . . 13

3.3 .1 Level Density Models . . . . . . . . . . . . . . . . . . . . . . . 13

3.3.2 Level Densities Above the Neutron Binding Energy . . . . . . . . . . . . . . . . . . . . 13

3.3.3 Systematic Behavior of Fermi Gas Level Density Parameters . . . . . . . . . . . . . 14

3.3.4 Level Densities Below the Neutron Binding Energy . . . . . . . . . . . . . . . . 15

3.4 Considerations Regarding the Exciton Model _ . . . . . . . . . . . . . . . . . 17

4 Calculated Cross Sections $\quad 17$

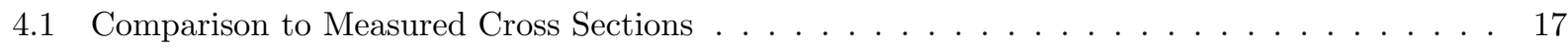

4.1 .1 Neutron capture cross sections . . . . . . . . . . . . . . . . . . 17

4.1 .2 Maxwellian averaged neutron capture cross sections . . . . . . . . . . . . . 19

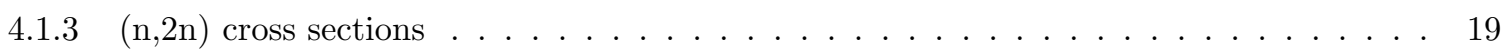

4.1.4 Neutron induced charged particle exit channel cross sections . . . . . . . . . . . . . 21

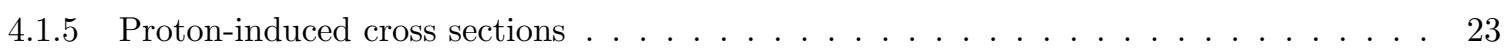

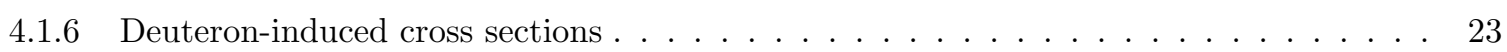

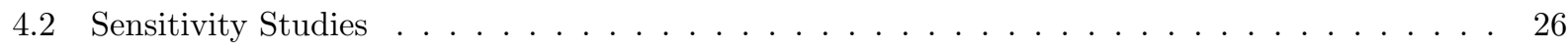

4.2 .1 Sensitivity to the Pre-Equilibrium Cross Section $\ldots \ldots \ldots \ldots \ldots \ldots$

4.2 .2 Sensitivity to the choice of Level Density Prescription . . . . . . . . . . . . . . . 26

4.2.3 Sensitivity to the Normalization of the $\gamma$-ray Transmission Coefficient . . . . . . . . . 28

4.2.4 Sensitivity to the Inclusion of Width Fluctuation Corrections . . . . . . . . . . . . 30 
4.3 Production and Destruction Cross Sections $\ldots \ldots \ldots \ldots \ldots \ldots$

5 Conclusions $\quad 32$

6 Acknowledgments 33

A Basic Nuclear Structure Data 36

A.1 New Scandium, Titanium-Vanadium, Chromium-Manganese, and Iron Detector Sets . . . . . 36

A.2 Binding and Separation Energies . . . . . . . . . . . . . . . . . . . . . 39

A.3 Q-values for Select Reactions . . . . . . . . . . . . . . . . . . . . . . . . . . . 43

A.4 Adopted Level Scheme Diagrams . . . . . . . . . . . . . . . . . . . . . . . . . . . . . . . 45

A.5 Nuclear Level Density Parameters . . . . . . . . . . . . . . . . . . . . . . . 60

B Modeled Cross Sections vs. Experiment 64

B.1 Modeled vs. Measured Cross Sections - $(n, \gamma)$. . . . . . . . . . . . . . . . . . . . . 64

B.2 Maxwellian averaged $(\mathrm{n}, \gamma)$ capture cross sections . . . . . . . . . . . . . . . . 68

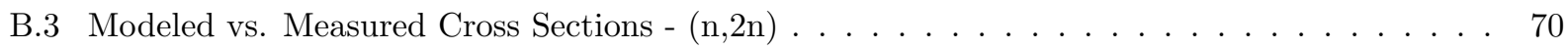

B.4 Modeled vs. Measured Cross Sections - $(\mathrm{n}, \mathrm{p})$. . . . . . . . . . . . . . . . . . . . . 72

B.5 Modeled vs. Measured Cross Sections - $(\mathrm{n}, \mathrm{np})+(\mathrm{n}, \mathrm{pn})$. . . . . . . . . . . . . . . . . . 76

B.6 Modeled vs. Measured Cross Sections - $(n, \alpha)$. . . . . . . . . . . . . . . . . . . . . 78

B.7 Modeled vs. Measured Cross Sections - $(\mathrm{n}, \mathrm{d})$. . . . . . . . . . . . . . . . . . . . . . . . . 81

B.8 Modeled vs. Measured Cross Sections - $\left(n, n^{\prime}\right)$. . . . . . . . . . . . . . . . . . . . . 83

B.9 Modeled vs. Measured Cross Sections - $(\mathrm{p}, \mathrm{n})$. . . . . . . . . . . . . . . . . . . . . . 85

B.10 Modeled vs. Measured Cross Sections - $(\mathrm{p}, 2 \mathrm{n})$. . . . . . . . . . . . . . . . . . . . . 88

B.11 Modeled vs. Measured Cross Sections - $(\mathrm{d}, \mathrm{n})$. . . . . . . . . . . . . . . . . . . . . . . 89

B.12 Modeled vs. Measured Cross Sections $-(\mathrm{d}, 2 \mathrm{n})$. . . . . . . . . . . . . . . . . . . . 90

C Modeled Cross Sections: Production and Destruction Channels 91

\section{List of Figures}

1 Total measured neutron cross sections vs Koning-Delaroche for select targets . . . . . . . . . 10

2 Measured $(\mathrm{p}, \mathrm{n})$ cross sections vs Koning-Delaroche for select targets . . . . . . . . . . . . . 11

3 Systematics for average total s-wave radiation width . . . . . . . . . . . . . . . . . 13

$4 \quad \chi^{2}$ linear fit to derived asymptotic level density parameters . . . . . . . . . . . . . . . 15

$5 \quad$ Constant temperature level density fits to the low lying spectroscopic levels of select nuclei . 16

6 Calculated vs. measured $(\mathrm{n}, \gamma)$ cross sections on ${ }^{45} \mathrm{Sc}{ }^{48} \mathrm{Ti},{ }^{52} \mathrm{Cr} \&{ }^{54} \mathrm{Fe}$. . . . . . . . . . . . 18

7 Calculated vs. recommended Maxwellian averaged (n, $\gamma)$ cross sections on ${ }^{45} \mathrm{Sc},{ }^{48} \mathrm{Ti},{ }^{52} \mathrm{Cr}, \&$

${ }^{54} \mathrm{Fe} \ldots \ldots \ldots \ldots \ldots \ldots \ldots \ldots$

8 Calculated vs. measured $(\mathrm{n}, 2 \mathrm{n})$ cross sections on ${ }^{45} \mathrm{Sc},{ }^{48} \mathrm{Ti},{ }^{52} \mathrm{Cr}, \&{ }^{54} \mathrm{Fe}$. . . . . . . . . 21

10 Calculated vs. measured (n,p) cross sections on ${ }^{45} \mathrm{Sc},{ }^{48} \mathrm{Ti},{ }^{52} \mathrm{Cr}, \&{ }^{54} \mathrm{Fe}$. . . . . . . . . . . 22

$9 \quad$ Observed and corrected ${ }^{48} \mathrm{Ti}(\mathrm{n}, 2 \mathrm{n})^{47} \mathrm{Ti}$ cross sections from (Dugersuren et al. 2005) . . . . . 22

11 Calculated vs. measured $(\mathrm{n}, \mathrm{np})+(\mathrm{n}, \mathrm{pn})$ cross sections on ${ }^{47-49} \mathrm{Ti}$. . . . . . . . . . . . . . . 23

12 Calculated vs. measured $(\mathrm{p}, \mathrm{n})$ cross sections on ${ }^{45} \mathrm{Sc},{ }^{48} \mathrm{Ti}, \&{ }^{52} \mathrm{Cr}$. . . . . . . . . . . . . . . . 24

13 Sample deuteron breakup fractions . . . . . . . . . . . . . . . . . . . . . 24

14 Calculated vs. measured $(\mathrm{d}, \mathrm{n})$ and $(\mathrm{d}, 2 \mathrm{n})$ cross sections . . . . . . . . . . . . . . . 25

15 Sensitivity of $(\mathrm{n}, 2 \mathrm{n})$ cross sections to pre-equilibrium matrix element . . . . . . . . . . . . 27

16 Sensitivity of $(n, p)$ and $(p, n)$ cross sections to pre-equilibrium matrix element . . . . . . . 28 
17 Sensitivity of $(\mathrm{n}, \gamma)$ and $(\mathrm{n}, 2 \mathrm{n})$ cross sections to the level density prescription $\ldots \ldots \ldots$

18 Sensitivity of $(\mathrm{n}, \mathrm{p})$ and $(\mathrm{p}, \mathrm{n})$ cross sections to the level density prescription $\ldots \ldots \ldots$

19 Sensitivity to a $\pm 30 \%$ adjustment of the experimental s-wave average photon width . . . . . . 31

20 Sensitivity to inclusion or exclusion of width fluctuation corrections . . . . . . . . . . . 32

21 Calculated cross sections directly affecting production and destruction of ${ }^{48} \mathrm{~V}$ and ${ }^{52} \mathrm{Mn} \ldots 33$

22 Measured vs. calculated cross sections for $(\mathrm{n}, \gamma)$ reactions $\ldots \ldots \ldots \ldots$. . . . . . . . 64

23 Measured vs. calculated maxwellian averaged $(\mathrm{n}, \gamma)$ cross sections . . . . . . . . . . . . 68

24 Measured vs. calculated cross sections for $(\mathrm{n}, 2 \mathrm{n})$ reactions $\ldots \ldots \ldots \ldots \ldots$

25 Measured vs. calculated cross sections for $(\mathrm{n}, \mathrm{p})$ reactions $\ldots \ldots \ldots \ldots \ldots$. . . . . . . . 72

26 Measured vs. calculated cross sections for $(\mathrm{n}, \mathrm{np})+(\mathrm{n}, \mathrm{pn})$ reactions $\ldots \ldots \ldots \ldots$

27 Measured vs. calculated cross sections for $(\mathrm{n}, \alpha)$ reactions $\ldots \ldots \ldots \ldots \ldots$

28 Measured vs. calculated cross sections for $(\mathrm{n}, \mathrm{d})$ reactions $\ldots \ldots \ldots \ldots$. . . . . . . . 81

29 Measured vs. calculated cross sections for $(\mathrm{n}, \mathrm{n})$ reactions $\ldots \ldots \ldots \ldots$. . . . . . . 83

30 Measured vs. calculated cross sections for $(\mathrm{p}, \mathrm{n})$ reactions $\ldots \ldots \ldots \ldots$. . . . . . . 85

31 Measured vs. calculated cross sections for $(\mathrm{p}, \mathrm{n})$ reactions on ${ }^{58} \mathrm{Fe} \ldots \ldots \ldots$. . . . . . . 87

32 Measured vs. calculated cross sections for $(\mathrm{p}, 2 \mathrm{n})$ reactions $\ldots \ldots \ldots \ldots$

33 Measured vs. calculated cross sections for $(\mathrm{d}, \mathrm{n})$ reactions $\ldots \ldots \ldots \ldots$

34 Measured vs. calculated cross sections for $(\mathrm{d}, 2 \mathrm{n})$ reactions $\ldots \ldots \ldots \ldots$. . . . . . . . 90

35 Production and destruction cross sections for $\mathrm{Sc}, \mathrm{Ti}, \& \mathrm{~V} \ldots \ldots \ldots$. . . . . . . . . . . 91

36 Production and destruction cross sections for $\mathrm{Cr}, \mathrm{Mn}, \& \mathrm{Fe} \ldots \ldots \ldots 7$

\section{List of Tables}

1 Cross sections available in previous detector set Ti0887 affecting the production of ${ }^{48} \mathrm{~V} \ldots \ldots \quad 5$

2 Cross sections available in previous detector sets $\mathrm{Cr} 0386$ and $\mathrm{Fe} 0987$ affecting the production

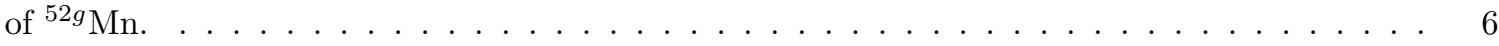

3 Comparison of our modeled (n, $\gamma)$ activation cross sections to measured values at $30 \pm 2 \mathrm{keV}$. 19

4 Comparison of modeled $(\mathrm{n}, 2 \mathrm{n})$ activation cross sections to measured values at $14.7 \pm 0.2 \mathrm{MeV} \quad 22$

$5 \quad$ Neutron induced reactions calculated for new scandium, titanium, chromium, and iron sets . 36

6 Charged-particle induced reactions calculated for new scandium, titanium, chromium, and

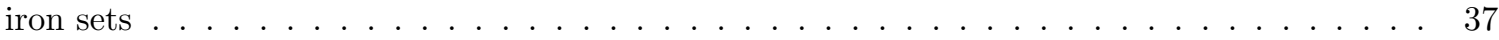

$7 \quad$ Spins, parities, binding energies, and separation energies $\ldots \ldots \ldots \ldots \ldots$

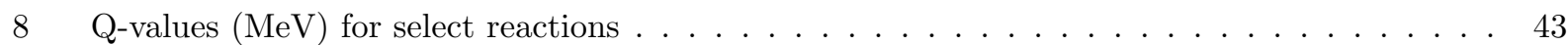

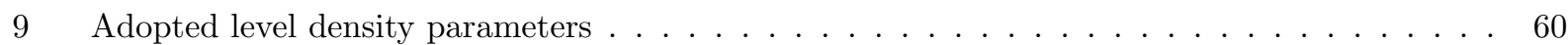




\subsection{Current Detector Sets}

\subsubsection{Titanium Charged Particle Detector Set}

Over the last 40 years a number of cross section detector sets have been developed at LLNL and LANL. Twenty-three neutron threshold detector sets and five charged particle sets are currently available.

The titanium set (Ti0887) is a neutron and charged particle activation cross section set used to calculate the activation of ${ }^{48} \mathrm{~V}\left(\tau_{1 / 2}=15.97\right.$ d) from stable titanium $\left({ }^{46-50} \mathrm{Ti}\right)$. It can also be used to calculate the production of ${ }^{46,47,48} \mathrm{Sc}$ from natural $\mathrm{Ti}$. It consists of 45 neutron- and 5 charged particle-induced cross sections. Ten of these are based on experimental measurements. The remainder are based on the ACTL Library (Gardner \& Howerton 1978). The set is summarized in (Nethaway 1998) and in Table 1. The two cross sections with asterisks in Table 1 are based on an experiment that measured ${ }^{\mathrm{Nat}} \mathrm{Ti}(\mathrm{t}, \mathrm{xn})^{48} \mathrm{~V}$, and was split into two individual cross sections based on calculations using the STAPRE HauserFeshbach code (Uhl \& Strohmaier 1976). The $(\mathrm{n}, \mathrm{X})$ reactions (or so-called "destruction" reactions) were estimated by subtracting the sum of the listed neutron induced reactions (i.e. (n, $\gamma)$, $(n, 2 n),(n, p)$, etc.) for a given target from a calculated total neutron reaction cross section and assigning the remainder to represent the $(n, X)$ reaction. The reactions listed as $(n, n p)$ are a sum of (n,np) and (n,pn).

\subsubsection{Chromium and Iron Charged Particle De- tector Sets}

The chromium set (Cr0386) is a neutron and charged particle set used to calculate activation of ${ }^{52 g} \mathrm{Mn}\left(\tau_{1 / 2}=5.59 \mathrm{~d}\right)$ from stable ${ }^{52} \mathrm{Cr}$, and consists of 31 neutron and seven charged particle cross sections. Of these five are from measurements, two were calculated, two were estimated, and the remaining 29 are from the ACTL Library (Gardner \& Howerton 1978). The set is summarized in (Nethaway 1998) and in Table 2. Again, the $(n, X)$ reactions are formed from a difference of a calculated reaction cross section and the sum of the neutron induced reactions on a given target isotope explicitly listed in the table.

A third detector set, Fe0987, was also developed to calculate the production of ${ }^{52 g} \mathrm{Mn}$ from stable ${ }^{54} \mathrm{Fe}$. It contains 11 neutron-induced cross sections, all but one from the ACTL library (Gardner \& Howerton 1978). The ${ }^{54} \mathrm{Fe}(\mathrm{n}, \mathrm{t}){ }^{52} \mathrm{Mn}$
Table 1: Cross sections available in previous detector set Ti0887 affecting the production of ${ }^{48} \mathrm{~V}$

\begin{tabular}{|c|c|c|}
\hline Reaction & Measured & ACTL \\
\hline${ }^{45} \mathrm{Sc}(\mathrm{n}, \gamma){ }^{46} \mathrm{Sc}$ & & $\bullet$ \\
\hline${ }^{45} \mathrm{Sc}(\mathrm{n}, \mathrm{X})$ & & $\bullet$ \\
\hline${ }^{46} \mathrm{Sc}(\mathrm{n}, 2 \mathrm{n}){ }^{45} \mathrm{Sc}$ & & $\bullet$ \\
\hline${ }^{46} \mathrm{Sc}(\mathrm{n}, \gamma){ }^{47} \mathrm{Sc}$ & & $\bullet$ \\
\hline${ }^{46} \mathrm{Sc}(\mathrm{n}, \mathrm{X})$ & & $\bullet$ \\
\hline${ }^{47} \mathrm{Sc}(\mathrm{n}, 2 \mathrm{n}){ }^{46} \mathrm{Sc}$ & & $\bullet$ \\
\hline${ }^{47} \mathrm{Sc}(\mathrm{n}, \gamma){ }^{48} \mathrm{Sc}$ & & $\bullet$ \\
\hline${ }^{47} \mathrm{Sc}(\mathrm{n}, \mathrm{X})$ & & $\bullet$ \\
\hline${ }^{48} \mathrm{Sc}(\mathrm{n}, 2 \mathrm{n}){ }^{47} \mathrm{Sc}$ & & $\bullet$ \\
\hline${ }^{48} \mathrm{Sc}(\mathrm{n}, \gamma){ }^{49} \mathrm{Sc}$ & & $\bullet$ \\
\hline${ }^{48} \mathrm{Sc}(\mathrm{n}, \mathrm{X})$ & & $\bullet$ \\
\hline${ }^{49} \mathrm{Sc}(\mathrm{n}, 2 \mathrm{n}){ }^{48} \mathrm{Sc}$ & & $\bullet$ \\
\hline${ }^{49} \mathrm{Sc}(\mathrm{n}, \mathrm{X})$ & & $\bullet$ \\
\hline${ }^{45} \mathrm{Ti}(\mathrm{n}, \gamma){ }^{46} \mathrm{Ti}$ & & $\bullet$ \\
\hline${ }^{45} \mathrm{Ti}(\mathrm{n}, \mathrm{X})$ & & $\bullet$ \\
\hline${ }^{46} \mathrm{Ti}(\mathrm{t}, \mathrm{n}){ }^{48} \mathrm{~V}\left({ }^{*}\right)$ & - & \\
\hline${ }^{46} \mathrm{Ti}(\mathrm{n}, 2 \mathrm{n}){ }^{45} \mathrm{Ti}$ & & $\bullet$ \\
\hline${ }^{46} \mathrm{Ti}(\mathrm{n}, \gamma){ }^{47} \mathrm{Ti}$ & & $\bullet$ \\
\hline${ }^{46} \mathrm{Ti}(\mathrm{n}, \mathrm{p}){ }^{46} \mathrm{Sc}$ & - & \\
\hline${ }^{46} \mathrm{Ti}(\mathrm{n}, \mathrm{np}){ }^{45} \mathrm{Sc}$ & & $\bullet$ \\
\hline${ }^{46} \mathrm{Ti}(\mathrm{n}, \mathrm{X})$ & & - \\
\hline${ }^{47} \mathrm{Ti}(\mathrm{d}, \mathrm{n}){ }^{48} \mathrm{~V}$ & $\bullet$ & \\
\hline${ }^{47} \mathrm{Ti}(\mathrm{t}, 2 \mathrm{n}){ }^{48} \mathrm{~V}(*)$ & • & \\
\hline${ }^{47} \mathrm{Ti}(\mathrm{n}, 2 \mathrm{n}){ }^{46} \mathrm{Ti}$ & & $\bullet$ \\
\hline${ }^{47} \mathrm{Ti}(\mathrm{n}, \gamma){ }^{48} \mathrm{Ti}$ & & $\bullet$ \\
\hline${ }^{47} \mathrm{Ti}(\mathrm{n}, \mathrm{p}){ }^{47} \mathrm{Sc}$ & - & \\
\hline${ }^{47} \mathrm{Ti}(\mathrm{n}, \mathrm{np}){ }^{46} \mathrm{Sc}$ & $\bullet$ & \\
\hline${ }^{47} \mathrm{Ti}(\mathrm{n}, \mathrm{X})$ & & - \\
\hline${ }^{48} \mathrm{Ti}(\mathrm{p}, \mathrm{n}){ }^{48} \mathrm{~V}$ & $\bullet$ & \\
\hline${ }^{48} \mathrm{Ti}(\mathrm{d}, 2 \mathrm{n}){ }^{48} \mathrm{~V}$ & $\bullet$ & \\
\hline${ }^{48} \mathrm{Ti}(\mathrm{n}, 2 \mathrm{n}){ }^{47} \mathrm{Ti}$ & & - \\
\hline${ }^{48} \mathrm{Ti}(\mathrm{n}, \gamma){ }^{49} \mathrm{Ti}$ & & - \\
\hline${ }^{48} \mathrm{Ti}(\mathrm{n}, \mathrm{p}){ }^{48} \mathrm{Sc}$ & - & \\
\hline${ }^{48} \mathrm{Ti}(\mathrm{n}, \mathrm{np}){ }^{47} \mathrm{Sc}$ & $\bullet$ & \\
\hline${ }^{48} \mathrm{Ti}(\mathrm{n}, \mathrm{X})$ & & - \\
\hline${ }^{49} \mathrm{Ti}(\mathrm{n}, 2 \mathrm{n}){ }^{48} \mathrm{Ti}$ & & • \\
\hline${ }^{49} \mathrm{Ti}(\mathrm{n}, \gamma){ }^{50} \mathrm{Ti}$ & & - \\
\hline${ }^{49} \mathrm{Ti}(\mathrm{n}, \mathrm{p}){ }^{49} \mathrm{Sc}$ & & $\bullet$ \\
\hline${ }^{49} \mathrm{Ti}(\mathrm{n}, \mathrm{np}){ }^{48} \mathrm{Sc}$ & & • \\
\hline${ }^{49} \mathrm{Ti}(\mathrm{n}, \mathrm{X})$ & & $\bullet$ \\
\hline${ }^{50} \mathrm{Ti}(\mathrm{n}, 2 \mathrm{n}){ }^{49} \mathrm{Ti}$ & & $\bullet$ \\
\hline${ }^{50} \mathrm{Ti}(\mathrm{n}, \mathrm{np}){ }^{49} \mathrm{Sc}$ & & • \\
\hline${ }^{50} \mathrm{Ti}(\mathrm{n}, \mathrm{X})$ & & - \\
\hline${ }^{47} \mathrm{~V}(\mathrm{n}, \gamma){ }^{48} \mathrm{~V}$ & & $\bullet$ \\
\hline${ }^{47} \mathrm{~V}(\mathrm{n}, \mathrm{X})$ & & $\bullet$ \\
\hline${ }^{48} \mathrm{~V}(\mathrm{n}, 2 \mathrm{n}){ }^{47} \mathrm{~V}$ & & • \\
\hline${ }^{48} \mathrm{~V}(\mathrm{n}, \gamma){ }^{49} \mathrm{~V}$ & & $\bullet$ \\
\hline${ }^{48} \mathrm{~V}(\mathrm{n}, \mathrm{X})$ & & - \\
\hline${ }^{49} \mathrm{~V}(\mathrm{n}, 2 \mathrm{n}){ }^{48} \mathrm{~V}$ & & $\bullet$ \\
\hline${ }^{49} \mathrm{~V}(\mathrm{n}, \mathrm{X})$ & & $\bullet$ \\
\hline
\end{tabular}


cross section is derived from a combination of experiment, calculations, and estimation. It is an average of two measurements made at 14 $\mathrm{MeV}$, the average being $0.0135 \mathrm{mb}$, and extrapolated linearly down to the threshold energy (Nethaway 1998).

All three sets are available on the world wide web at http://nuclear.llnl.gov/CNP/nads/ main.html.

\subsection{The Need for New Detector Sets}

The motivations for revisiting the detector sets for radiochemistry are many. The current sets were often developed based on "best guess" and "experience" from a limited amount of experimental data over a 40 year period. Often a single measurement at $14 \mathrm{MeV}$ guided the evaluation of a critical $(n, 2 n)$ cross section, with an assumed shape that would rise from a calculated threshold, and then adjusted to match the experimental point at $14 \mathrm{MeV}$.

Of the 92 cross sections in the three sets, only 16 are based on experimental measurements. Of the remaining cross sections, most were taken from a cross section library developed 25 years ago. A few cross sections were estimated based on systematics.

Several of the isotopes included as targets in these cross section sets have long-lived isomers, but the sets only include activation cross sections. In some cases, the cross section for a given reaction for an isomer target can differ substantially from the cross section for the ground state target, particularly when the spin of the isomer is much larger or smaller than the spin of the ground state nucleus.

Most importantly, the adoption of $(\mathrm{n}, \mathrm{X})$ "destruction cross sections" overlooks an important question: what is the largest of these cross sections and to which final state is it assigned? Our modelling effort has determined that the $(n, p)$ cross section for three long lived radioactivities ${ }^{44} \mathrm{Sc}$, ${ }^{48} \mathrm{~V}$, and ${ }^{52 g} \mathrm{Mn}$, is the largest destruction channel. This should have important consequences on reaction networks using these cross sections. In the previous sets, these cross sections were not explicitly calculated or assigned.

In the nearly two decades since these cross section sets were last evaluated, many new cross section measurements have been performed, and several efforts have been made to develop consistent approaches to modeling nuclear reaction cross sections (RIPL 1998). The basic nuclear structure
Table 2: Cross sections available in previous detector sets Cr0386 and Fe0987 affecting the production of ${ }^{52 g} \mathrm{Mn}$.

\begin{tabular}{|c|c|c|c|c|}
\hline Reaction & Meas. & Calc. & Est. & 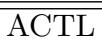 \\
\hline${ }^{48} \mathrm{Cr}(\mathrm{n}, \mathrm{X})$ & & & & $\bullet$ \\
\hline${ }^{49} \mathrm{Cr}(\mathrm{n}, 2 \mathrm{n}){ }^{48} \mathrm{Cr}$ & & & & $\bullet$ \\
\hline${ }^{49} \mathrm{Cr}(\mathrm{n}, \gamma){ }^{50} \mathrm{Cr}$ & & & & $\bullet$ \\
\hline${ }^{49} \mathrm{Cr}(\mathrm{n}, \mathrm{p}){ }^{49} \mathrm{~V}$ & & & & $\bullet$ \\
\hline${ }^{49} \mathrm{Cr}(\mathrm{n}, \mathrm{np}){ }^{48} \mathrm{~V}$ & & & & $\bullet$ \\
\hline${ }^{49} \mathrm{Cr}(\mathrm{n}, \mathrm{X})$ & & & & $\bullet$ \\
\hline${ }^{50} \mathrm{Cr}(\mathrm{d}, \mathrm{n}){ }^{51} \mathrm{Mn}$ & & & • & \\
\hline${ }^{50} \mathrm{Cr}(\mathrm{d}, \mathrm{a}){ }^{48} \mathrm{~V}$ & • & & & \\
\hline${ }^{50} \mathrm{Cr}(\mathrm{n}, 2 \mathrm{n}){ }^{49} \mathrm{Cr}$ & & & & $\bullet$ \\
\hline${ }^{50} \mathrm{Cr}(\mathrm{n}, \gamma){ }^{51} \mathrm{Cr}$ & & & & $\bullet$ \\
\hline${ }^{50} \mathrm{Cr}(\mathrm{n}, \mathrm{np}){ }^{49} \mathrm{~V}$ & $\bullet$ & & & \\
\hline${ }^{50} \mathrm{Cr}(\mathrm{n}, \mathrm{t}){ }^{48} \mathrm{~V}$ & $\bullet$ & & & \\
\hline${ }^{50} \mathrm{Cr}(\mathrm{n}, \mathrm{X})$ & & & & $\bullet$ \\
\hline${ }^{51} \mathrm{Cr}(\mathrm{d}, \mathrm{n}){ }^{52} \mathrm{Mn}$ & & & $\bullet$ & \\
\hline${ }^{51} \mathrm{Cr}(\mathrm{n}, 2 \mathrm{n}){ }^{50} \mathrm{Cr}$ & & & & $\bullet$ \\
\hline${ }^{51} \mathrm{Cr}(\mathrm{n}, \gamma){ }^{52} \mathrm{Cr}$ & & & & $\bullet$ \\
\hline${ }^{51} \mathrm{Cr}(\mathrm{n}, \mathrm{X})$ & & & & $\bullet$ \\
\hline${ }^{52} \mathrm{Cr}(\mathrm{p}, \mathrm{n}){ }^{52} \mathrm{Mn}$ & $\bullet$ & & & \\
\hline${ }^{52} \mathrm{Cr}(\mathrm{d}, \mathrm{n}){ }^{53} \mathrm{Mn}$ & & $\bullet$ & & \\
\hline${ }^{52} \mathrm{Cr}(\mathrm{d}, 2 \mathrm{n}){ }^{52} \mathrm{Mn}$ & $\bullet$ & & & \\
\hline${ }^{52} \mathrm{Cr}(\mathrm{n}, 2 \mathrm{n}){ }^{51} \mathrm{Cr}$ & & & & $\bullet$ \\
\hline${ }^{52} \mathrm{Cr}(\mathrm{n}, \gamma){ }^{53} \mathrm{Cr}$ & & & & $\bullet$ \\
\hline${ }^{52} \mathrm{Cr}(\mathrm{n}, \mathrm{X})$ & & & & $\bullet$ \\
\hline${ }^{53} \mathrm{Cr}(\mathrm{p}, 2 \mathrm{n}){ }^{52} \mathrm{Mn}$ & & $\bullet$ & & \\
\hline${ }^{51} \mathrm{Mn}(\mathrm{n}, \gamma){ }^{52} \mathrm{Mn}$ & & & & $\bullet$ \\
\hline${ }^{51} \mathrm{Mn}(\mathrm{n}, \mathrm{X})$ & & & & $\bullet$ \\
\hline${ }^{52} \mathrm{Mn}(\mathrm{n}, 2 \mathrm{n}){ }^{51} \mathrm{Mn}$ & & & & $\bullet$ \\
\hline${ }^{52} \mathrm{Mn}(\mathrm{n}, \gamma){ }^{53} \mathrm{Mn}$ & & & & $\bullet$ \\
\hline${ }^{52} \mathrm{Mn}(\mathrm{n}, \mathrm{X})$ & & & & • \\
\hline${ }^{53} \mathrm{Mn}(\mathrm{n}, 2 \mathrm{n}){ }^{52} \mathrm{Mn}$ & & & & $\bullet$ \\
\hline${ }^{53} \mathrm{Mn}(\mathrm{n}, \mathrm{X})$ & & & & $\bullet$ \\
\hline${ }^{53} \mathrm{Fe}(\mathrm{n}, 2 \mathrm{n}){ }^{52} \mathrm{Fe}$ & & & & $\bullet$ \\
\hline${ }^{53} \mathrm{Fe}(\mathrm{n}, \gamma){ }^{54} \mathrm{Fe}$ & & & & $\bullet$ \\
\hline${ }^{53} \mathrm{Fe}(\mathrm{n}, \mathrm{p}){ }^{53} \mathrm{Mn}$ & & & & $\bullet$ \\
\hline${ }^{53} \mathrm{Fe}(\mathrm{n}, \mathrm{np}){ }^{52} \mathrm{Mn}$ & & & & $\bullet$ \\
\hline${ }^{53} \mathrm{Fe}(\mathrm{n}, \mathrm{X})$ & & & & $\bullet$ \\
\hline${ }^{54} \mathrm{Fe}(\mathrm{n}, \mathrm{t}){ }^{52} \mathrm{Mn}$ & $\bullet$ & $\bullet$ & $\bullet$ & \\
\hline${ }^{54} \mathrm{Fe}(\mathrm{n}, 2 \mathrm{n}){ }^{53} \mathrm{Fe}$ & & & & $\bullet$ \\
\hline${ }^{54} \mathrm{Fe}(\mathrm{n}, \mathrm{p}){ }^{54} \mathrm{Mn}$ & & & & $\bullet$ \\
\hline${ }^{54} \mathrm{Fe}(\mathrm{n}, \mathrm{np}){ }^{53} \mathrm{Mn}$ & & & & $\bullet$ \\
\hline${ }^{54} \mathrm{Fe}(\mathrm{n}, \mathrm{X})$ & & & & $\bullet$ \\
\hline
\end{tabular}


data has been greatly improved. Finally, there are more accurate methods of calculating and estimating cross sections for which we have no data.

\subsection{Proposed Sc, Ti, Cr, and Fe Detector Sets}

We consider as targets isotopes of the elements $\mathrm{Sc}, \mathrm{Ti}, \mathrm{V}, \mathrm{Cr}, \mathrm{Mn}$, and $\mathrm{Fe}(21 \leq \mathrm{Z} \leq 26)$ with neutron numbers in the range $20 \leq \mathrm{N} \leq 38$. This includes any long-lived isomers with half-lives greater than $1 \mu \mathrm{s}$, which were not included in the original sets. We have calculated nuclear reaction cross sections for incident neutrons, protons, and deuterons on these targets with laboratory incident particle energies ranging from $0.01 \mathrm{keV}$ to 20 $\mathrm{MeV}$. These compound systems are then allowed to decay through the reaction channels shown in Appendix 5.

The reason for including many more isotopes and reactions than the original sets is to account for the various possible destruction reactions that are significant in this mass range. In general, the current RADCHEM detector sets, and especially the charged-particle sets, were developed with special attention paid to the production reactions.

In addition to these reactions, we have calculated several neutron and proton induced cross sections on stable calcium and iron targets in an effort to produce a larger set of calculated cross sections in the local region of interest that can be compared against experimental cross section data.

Our goal is to develop a consistent set that reproduces, as closely as possible, measured cross sections on targets in the local region of interest. To do this we develop local systematics for the many input quantities used in the theoretical reaction modeling calculations. These systematics are based on experimental data that are often only available for compound nuclear systems formed from a stable target plus a neutron. Of course, we use experimental data whenever it is available, but reactions proceeding through unstable systems are unavoidable in radiochemistry. Short of developing new experimental techniques to measure cross sections on unstable targets, our only hope of reproducing measured activity from UGT shots, and addressing the uncertainty associated with the nuclear cross sections, is to develop cross section sets that reproduce well the measured cross sections in the region of interest.

In $\S 2$ we describe the theoretical techniques used in the modeling effort. $\S 3$ describes the input parameters. $\S 4$ gives results. We conclude with $\S 5$.

\section{Nuclear Reaction Theory}

\subsection{Reaction Mechanisms}

Conceptually, we consider nuclear reaction mechanisms to be of two general types, direct processes and compound processes. Direct processes can be pictured as simple interactions of the incident particle with the nuclear potential of the target nucleus. They proceed on a rapid time scale (of order $\sim 10^{-22} \mathrm{~s}$ ), and the reaction products are often highly peaked in the incident particle direction. Compound processes are pictured as complicated interactions proceeding over a much longer timescale $\left(10^{-15}-10^{-18} \mathrm{~s}\right)$ in which the reaction is mediated by the formation of a "compound nucleus", with the excitation energy of the incident particle being statistically "shared" with the ensemble of nucleons in the target over all energetically allowed degrees of freedom. The reaction products are largely isotropic. Compound nuclear reactions proceed through resonances, which correspond to nuclear states above the bound region, while direct reactions proceed through smooth potential terms. Other intermediate reaction mechanisms may exist between these two extremes. We refer to these as "precompound" nuclear processes.

Over the energy range of interest to this project, a few $\mathrm{keV}$ to $20 \mathrm{MeV}$, we consider pre-compound and compound nuclear processes, with the precompound reactions operating principally above $10 \mathrm{MeV}$ of incident particle excitation energy.

\subsection{Hauser-Feshbach Statistical Model}

A traditional theoretical approach to compound nuclear reactions is the statistical or HauserFeshbach model. This model is valid only for high level densities in the compound nucleus, allowing one to use energy averaged transmission coefficients $T$, which describe absorption via an imaginary part in the (optical) nucleon-nucleus potential. For the reaction I (in state $\mu)+j \rightarrow k+\mathrm{L}$ (in state $\nu$ ), with $\mathrm{I}^{\mu}+j$ interacting with centerof-mass energy $\mathrm{E}_{j}^{\mu}$ (in $\mathrm{MeV}$ ), the average cross section is given by

$$
\sigma_{j k}^{\mu \nu}\left(E_{j}^{\mu}\right)=\frac{\pi \lambda_{j}^{2}}{g_{I}^{\mu} g_{j}} \sum_{J, \pi} g_{J} \frac{T_{j}^{\mu}\left(J^{\pi}\right) T_{k}^{\nu}\left(J^{\pi}\right)}{T_{t o t}\left(J^{\pi}\right)} W\left(J^{\pi}\right)
$$

where the summation extends over all compound nuclear spins and parities $J^{\pi}, \mu$ and $\nu$ are states in the target and product $(=0$ for the ground 
state, 1 for the $1^{\text {st }}$ excited state, etc.). The cross section has units of area, described by $\pi \lambda_{j}^{2}=$ $0.6566\left(\hat{A}_{j} E_{j}^{\mu}\right)^{-1}$ barns, with $\hat{A}_{j}=\left(A_{I} A_{j}\right) /\left(A_{I}+\right.$ $A_{j}$ ) being the reduced mass in atomic mass units and $E_{j}^{\mu}$ is the center of mass energy in units of $\mathrm{MeV} . \lambda_{j}$ is the wavelength related to the wave number $k_{j}$ in the target plus incident particle channel by $\lambda_{j}=1 / k_{j}$ The statistical weights are given by $g_{y}^{x}=\left(2 J_{y}^{x}+1\right)$. Items without superscripts refer to the compound nucleus.

The transmission coefficients in the numerator are the total transmission coefficient for forming the state $J^{\pi}$ in the compound nucleus $I^{\mu}+j$ at energy $E_{j}^{\mu}$, given by $T_{j}^{\mu}\left(J^{\pi}\right) . T_{k}^{\nu}\left(J^{\pi}\right)$ is the same as $T_{j}^{\mu}\left(J^{\pi}\right)$ but for the pair $L^{\nu}+k$ at energy $E_{k}^{\nu}$. Implicit in these definitions is a sum over all possible $l$-waves and channel spins, i.e.

$$
T_{j}^{\mu}\left(J^{\pi}\right)=\sum_{l, s} T_{j}^{\mu}\left(J^{\pi}, l, s\right)
$$

where $l$ is any partial wave number (orbital angular momentum) that can couple the state $\mu$ to the compound nuclear state having spin and parity $J^{\pi}$ subject to quantum mechanical selection rules and $s$ is the vector sum of the spins $J_{I}^{\mu}$ and $J_{j}$. Hence $s$ takes on all integer (or half-integer) numbers from $\left|J_{I}^{\mu}-J_{j}\right|$ to $J_{I}^{\mu}+J_{j}$.

$T_{t o t}$ represents the sum of transmission coefficients over all possible decay channels (i.e. for all particles and photons). The cross section for the formation of species $L$, regardless of its state $\nu$, is obtained by summing Eq. [1] over all bound states $\nu$ of $L$ for which the reaction is energetically allowed.

When evaluating these sums, if energies become of interest which exceed the highest discrete excited state for which energy, spin, and parity are explicitly known, a nuclear level density formula must be employed. Specifically, the definitions for the transmission coefficients $T_{j}\left(J^{\pi}\right), T_{k}\left(J^{\pi}\right)$, and $T_{\text {tot }}\left(J^{\pi}\right)$ must be modified, for example:

$$
\begin{aligned}
& T_{k}\left(J^{\pi}\right)=\sum_{\nu=0}^{\omega} T_{k}^{\nu}\left(J^{\pi}\right)+ \\
& \int_{\xi_{L}^{\omega}}^{\xi_{L}^{\max }} \int_{J^{\nu} \pi^{\nu}} T_{k}^{\nu}\left(\xi_{L}^{\nu}, J^{\pi}\right) \rho\left(\xi_{L}^{\nu}, J^{\nu}, \pi^{\nu}\right) d \xi_{L}^{\nu} d \pi^{\nu} d J^{\nu}
\end{aligned}
$$

where for the nucleus $L, \xi_{L}^{\omega}$ is the energy of the highest excited state, $\omega$, of known energy, spin, and parity; $\xi_{L}^{\max }=E_{k}^{0}=E_{j}^{0}+Q_{j k}$ is the maximum excitation energy available, and $\rho\left(\xi_{L}^{\nu}, J^{\nu}, \pi^{\nu}\right)$ is the density of states per unit energy of spin and parity $J^{\nu}$ and $\pi^{\nu}$ at the excitation energy $\xi_{L}^{\nu}$. The above integral approximates a summation and is subject to the same quantum mechanical restrictions implied in the definition of the transmission function.

\subsection{Width Fluctuations}

In addition to the transmission coefficients for particles and photons and level densities required for Eq. [1], width fluctuation corrections (WFC, $W\left(J^{\pi}\right)$ in Eq. [1]) must also be employed. They define the correlation factors with which all partial channels of incoming particle $j$ and outgoing particle $k$, passing through excited state $(E, J, \pi)$, have to be multiplied. The major effect is to enhance the elastic channel and accordingly decrease the other open channels. These effects are most often observed at or near channel opening energies when i.e. a $(\mathrm{p}, \gamma)$ and a $(\mathrm{p}, \mathrm{n})$ channel compete and the weaker $(p, \gamma)$ channel is enhanced. Above a few $\mathrm{MeV}$ of excitation energy, when many competing channels are open, WFC's can be neglected.

The exact expression for the WFC, obtained with the Gaussian orthogonal ensemble (GOE) approach, requires the evaluation of a triple integral and to date has been considered much to costly to apply in nuclear cross section calculations. Several approximations have been developed, the most popular ones are the Moldauer model (Moldauer 1976), and the HRTW model (Hofmann et al. 1975). We use the Moldauer model approximation in this study. For a detailed description of the full (GOE) treatment and a comparison with the Moldauer and HRTW approximation models mentioned above, see (Hilaire Lagrange \& Koning 2003).

\subsection{Pre-Compound Processes}

For excitation energies starting around $10 \mathrm{MeV}$, pre-compound processes become important. We account for the pre-equilibrium emission of particles from the first compound nucleus using a simple exciton model. In the pre-equilibrium stage of the reaction, particle emission is assumed to be the only decay mode. Once the pre-equilibrium cross section leading to the appropriate final state has been calculated, the total pre-equilibrium cross section is subtracted from the reaction cross section. The reaction then proceeds through the usual statistical channels (Eq. [1]). Pre-compound processes are generally important only for the first compound nucleus. Subsequent (higher chance) processes are treated as sequential evaporation 
steps. After the Hauser-Feshbach portion of the calculation is complete, the pre-equilibrium cross section leading to the desired final state is added back to the statistical model cross section.

\subsection{The STAPRE Hauser-Feshbach Reac- tion Code}

We adopt the statistical model code STAPRE (STAatistical-PREequilibrium) to model our cross sections (Uhl \& Strohmaier 1976). It embodies all of the physical models discussed above. The version of the code we use is STAPRE-H95 (Avrigeanu \& Avrigeanu 1976), available from the NEA web site. We have made several modifications, primarily to the level density routines. Prior versions of the code were used to develop parts of the existing RADCHEM data sets (Vonach 1982).

In the following we discuss the important ingredients of statistical model calculations, and the methods used to estimate them. These are the requisite nuclear structure data (energies, spins, and parities of the ground states and all known excited states, as well as detailed branching ratios for the gamma-ray cascade from excited to lowlying states), the width fluctuation corrections, the pre-compound cross section, the particle and $\gamma$-transmission coefficients, and the nuclear level densities of all nuclei involved in the reaction. The reliability of such calculations is chiefly determined by the accuracy with which these components can be evaluated.

\section{Inputs Required for the Hauser-Feshbach Model}

\subsection{Nuclear Structure Data}

\subsubsection{Nuclear Masses and $J^{\pi}$ Assignments}

We adopt for nuclear masses the experimental mass excess values of (Möller et al. 1995). Spin and parity assignments are from the Evaluated Nuclear Structure Data File (ENSDF 2003). We present in Table 3 (Appendix 7) the binding energies (in $\mathrm{MeV}$ ) calculated from the adopted masses, the ground state spins and parities, and the separation energies for neutrons, protons, alphaparticles, and deuterons for the nuclei included in this study. In Table 4 (Appendix 8), we provide reaction $\mathrm{Q}$-values for the cross sections calculated in this study.

\subsubsection{Nuclear Level Schemes}

The nuclear structure data needed to model the gamma-ray cascade in this study was adopted from the file BUDAPEST.DAT (RIPL 1998). For some of the isotopes, additional evaluation was performed by R. Bauer (Bauer 2001). Shown in Appendix A.4 are a selection of the modified adopted nuclear levels, including level energy, spin and parity assignments, and their gamma-cascade branching ratios. The number of excited levels adopted for each nucleus is given as the quantity "N" in Table 5 (Appendix 9). For the unmodified isotopes, this was the number for which energy spin and parity were unambiguously assigned in the BUDAPEST file. Nuclei for which only a ground state was used are indicated by $\mathrm{N}=0$.

\subsection{Transmission Coefficients}

\subsubsection{Transmission Coefficients for Particles}

We restrict our attention in this modeling effort to reactions with incident neutrons, protons, and deuterons. For the charged particle cross sections to be included in the new sets, we adopt the 15 experimental results used in the previous detector sets (Nethaway 1998). We present modeled results for $(\mathrm{p}, \mathrm{n}),(\mathrm{p}, 2 \mathrm{n}),(\mathrm{d}, \mathrm{n})$, and $(\mathrm{d}, 2 \mathrm{n})$ reactions on all isotopes included as targets. We do not include any triton or alpha-induced reactions.

\subsubsection{The Optical Potential of Koning and De- laroche}

For the calculation of the neutron and proton particle transmission coefficients, we use the optical model developed by (Koning \& Delaroche 2001). Although they have tuned their parameters to fit data for many different species (see their Tables 6 and 7 ), we decided to use the global nucleonnucleon optical model potential (OMP), as it gives a very satisfactory fit to measured total cross section data for neutrons and protons in the range of interest to us (e.g. Sc-Mn). Specifically, we adopt the potential depth parameters and Fermi energies for the neutron and proton global OMP defined in their Section 5.2, tables 14 and 15. The particle transmission coefficients were generated by the optical model code ECIS-95 (Raynal 1996). Although designed for coupled channel calculations, we used the code in a spherical optical model mode. 

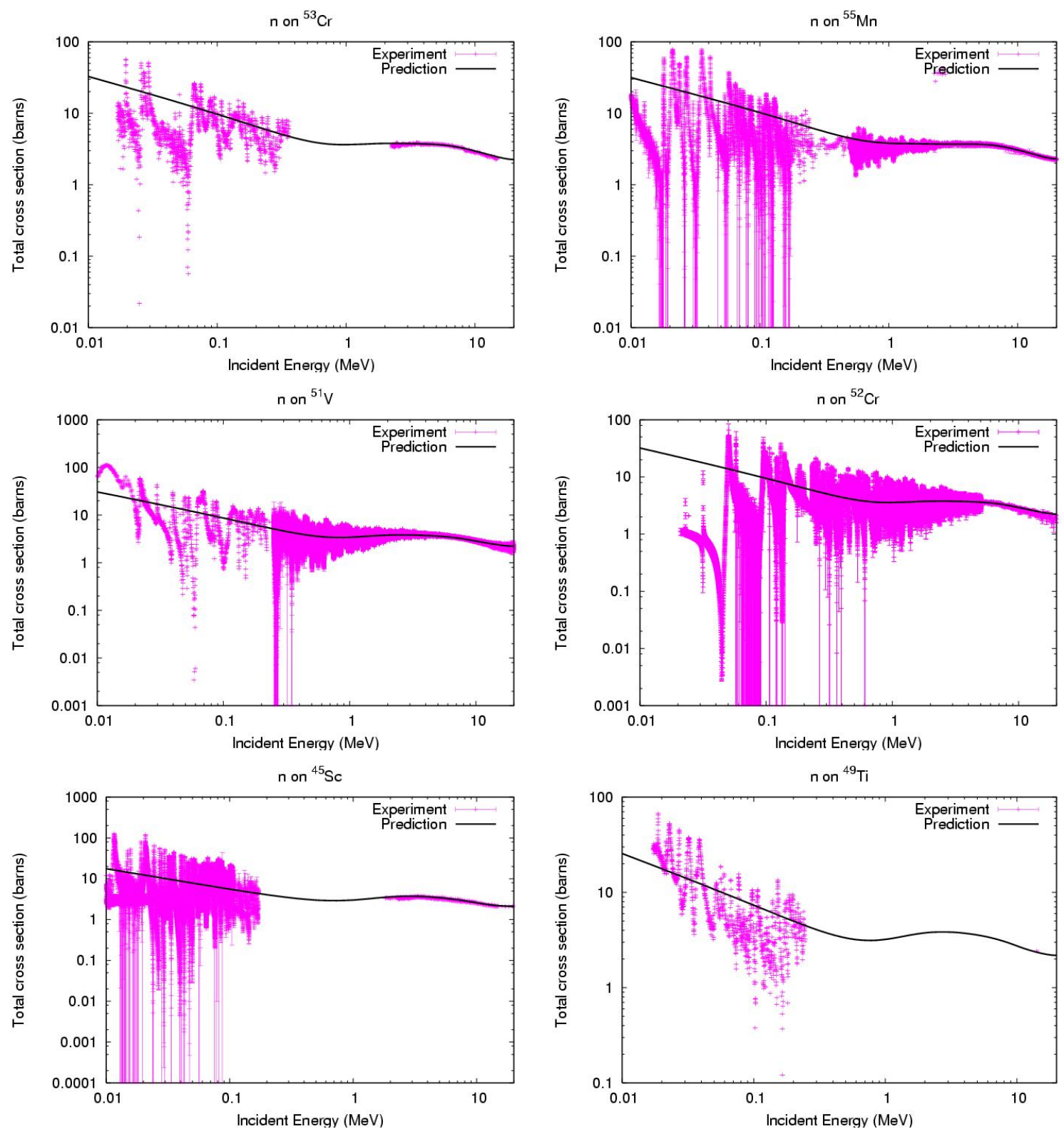

Fig. 1.- Total measured neutron cross sections vs Koning-Delaroche for select targets. Measured values were obtained from (CSISRS 2003). The optical model prediction is indicated by the solid black line.

\subsubsection{Evaluation of the Neutron and Proton Op- tical Potential}

We present in Figure (1) results of the Koning \& Delaroche optical model compared to measured total neutron cross sections for select targets in our range of interest. Additional total neutron cross section data sets are also available in this region, although they generally consist of fewer data points (in some cases a single point at 14 $\mathrm{MeV})$. There is a considerable amount of data available for iron targets. In each instance, the optical model closely replicates the experimental data. The optical model result predicts average cross sections, not the resonance structure evident in the figures at lower energies. For additional comparisons, see (Koning \& Delaroche 2001).

Figure (2) illustrates the Koning-Delaroche proton potential vs. experimental data for the same targets used for the total neutron cross section comparison. Plotted are $(\mathrm{p}, \mathrm{n})$ cross sections from experiment and calculated using the Koning \& Delaroche optical model $\left(\mathrm{KD} \sigma_{\mathrm{pn}}\right.$, the blue 

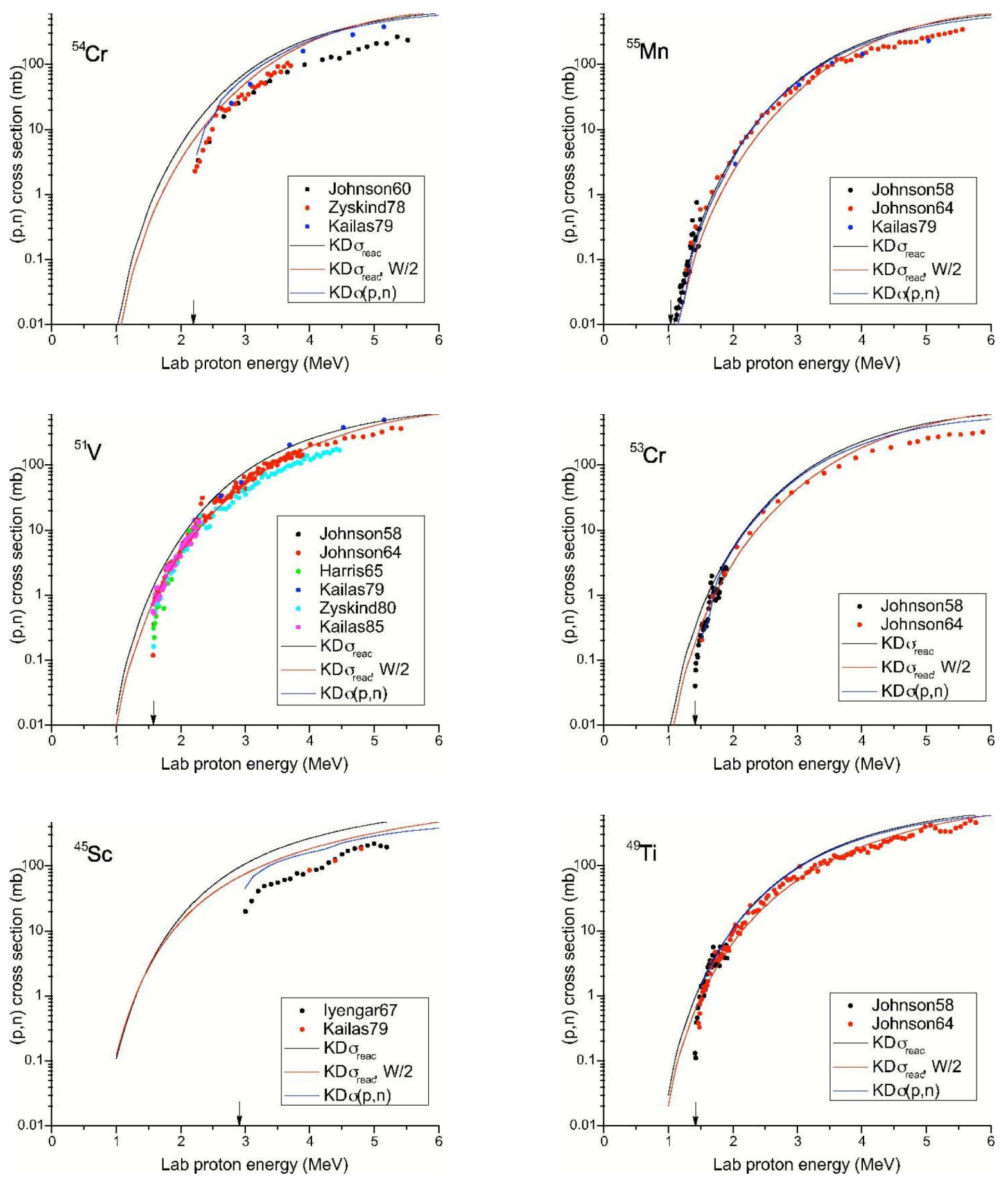

Fig. 2.- Measured (p,n) cross sections, approximately equal to the total proton cross section at low energies, vs Koning-Delaroche for select targets. Solid black lines represent the total reaction cross section for incident protons obtained from the optical potential. Red lines represent this same quantity, but with the imaginary potential depth reduced by a factor of two. Blue lines represent the (p,n) cross section.

line). These are not total proton cross sections, but they are closely related. In the energy region above the coulomb barrier near the proton reaction threshold (indicated by the black arrow on the incident proton energy axis) where the neutron channel is open (i.e. where the proton just gets absorbed by the target nucleus), the only particle emmission possible is a neutron, the proton 
(and heavier charged particles) being blocked by the coulomb barrier. In this energy range the $(\mathrm{p}, \mathrm{n})$ cross section is a good approximation to $\sigma_{\text {reac }}$, the proton reaction cross section also calculated with the KD OM (black solid line). We also plot the same quantity but with the imaginary part of the potential divided by two (red line). For half of the systems shown, KD $\sigma_{\text {reac }}$ lies above $\sigma_{\mathrm{pn}}$ by roughly a factor of two. Reducing the imaginary part of the potential does not necessarily cut the reaction cross section in half (there are instances in this region where it can actually increase it).

The inverse reaction, (n,p), populates the same compound nucleus as the $(\mathrm{p}, \mathrm{n})$ reaction and so utilizes the same optical potentials in the statistical model calculation (Eq. [1]), each enter as products and sums in the numerator and denomenator respectively. The experimental evidence for the $(\mathrm{p}, \mathrm{n})$ reactions suggest that our proton optical potential may be too high by as much as a factor of two in this region. Calculations of $(n, p)$ cross sections using these potentials will reflect the same behavior. Since these are the dominant destruction reactions for the radioisotopes of interest here, ${ }^{48} \mathrm{~V}$ and ${ }^{52 g} \mathrm{Mn}$, this could have important implications for reaction networks that use these cross sections.

\subsubsection{The Alpha and Deuteron Optical Poten- tials}

We have included possible alpha and deuteron exit channels in this modeling effort. For the alpha particles, we use the optical potential of (McFadden \& Satchler 1966), and for deuterons we use that of (Perey \& Perey 1963), as encoded in the SCAT2 subroutine of STAPRE-H95.

We do not include a quality analysis of these potentials in this report. The deuteron and alpha exit channels are small when compared to the dominant channel, accounting for less than $10 \%$ of the total reaction cross section in all cases, less than $5 \%$ in most. Therefore, any sensitivity to the alpha and deuteron potentials will only be apparent in these weak exit channels. Additionally, somewhat reasonable agreement with the experimental $(\mathrm{n}, \alpha)$ cross sections provides us with some degree of confidence in the alpha potential (see Appendix B.6). The results for deuterons in the exit channel are mixed, but many rely on a single data point at one energy that exhibits a large error bar. See Appendix B.7 for examples.

\subsubsection{Transmission Coefficients for Photons}

For the calculation of the gamma ray transmission coefficients, we use a simple model where the transmission coefficient depends only on the multipole type (XL) and the transition energy $(\epsilon)$, as encoded in STAPRE (Avrigeanu \& Avrigeanu 1976). They are related to the gamma ray strength function $f_{X L}^{\gamma}(\epsilon)$ by

$$
T_{X L}^{\gamma}(\epsilon)=2 \pi \epsilon^{2 L+1} f_{X L}^{\gamma}(\epsilon)
$$

The energy dependence of the strength function was determined using the GDR model with simple Lorentzian line shapes. In particular, the E1 strength function is given by

$f_{E 1}^{\gamma}(\epsilon)=\mathcal{N} \zeta \sigma_{G} \frac{\Gamma_{G}^{2} \epsilon}{\left(\epsilon^{2}-E_{G}^{2}\right)^{2}+\left(\Gamma_{G} \epsilon\right)^{2}}\left(\mathrm{MeV}^{-3}\right)$

where $E_{G}, \Gamma_{G}$, and $\sigma_{G}$ are the energy, width, and peak cross section of the Giant Dipole Resonance given in $\mathrm{MeV}$ and $\mathrm{mb}$ respectively. The constant $\zeta$ is $8.674 \times 10^{-8} \mathrm{mb}^{-1} \cdot \mathrm{MeV}^{-2}$. The GDR parameters are given by

$$
\begin{aligned}
E_{G} & =\frac{80 \mathrm{MeV}}{A_{C}^{1 / 3}} \\
\Gamma_{G} & =5 \mathrm{MeV} \\
\sigma_{G} & =A_{C} \frac{13 \mathrm{mb}}{5}
\end{aligned}
$$

where $A_{C}$ is the mass number of the compound nucleus. We also include M1, E2, and M2 transitions. Their transmission coefficients are simply proportional to $\epsilon^{2 L+1}$, and therefore their strength functions are constants. In particular,

$$
\begin{aligned}
f_{M 1}^{\gamma}(\epsilon) & =3.1 \times 10^{-1} A_{C}^{-2 / 3} f_{E 1}^{\gamma}\left(S_{n}\right) \\
f_{E 2}^{\gamma}(\epsilon) & =7.2 \times 10^{-7} A_{C}^{2 / 3} f_{E 1}^{\gamma}\left(S_{n}\right) \\
f_{M 2}^{\gamma}(\epsilon) & =2.2 \times 10^{-7} f_{E 1}^{\gamma}\left(S_{n}\right)
\end{aligned}
$$

where $S_{n}$ is the neutron separation energy. In all cases, $\mathrm{E} 1$ is the dominant multipole radiation.

The factor $\mathcal{N}$ appearing in Eq. 4 is a normalization constant, determined by fitting the average total $s$-wave radiation width at the neutron binding energy,

$$
\begin{gathered}
\left\langle\Gamma_{\gamma}\right\rangle_{0}=\frac{J+1}{2 J+1}\left\langle\Gamma_{\gamma}\left(B_{n}, J+\frac{1}{2}\right)\right\rangle \\
+\frac{J}{2 J+1}\left\langle\Gamma_{\gamma}\left(B_{n}, J-\frac{1}{2}\right)\right\rangle \\
\Gamma_{\gamma}(E, J)=\frac{T_{\gamma}(E, J)}{2 \pi \rho(E, J)}(\mathrm{meV})
\end{gathered}
$$




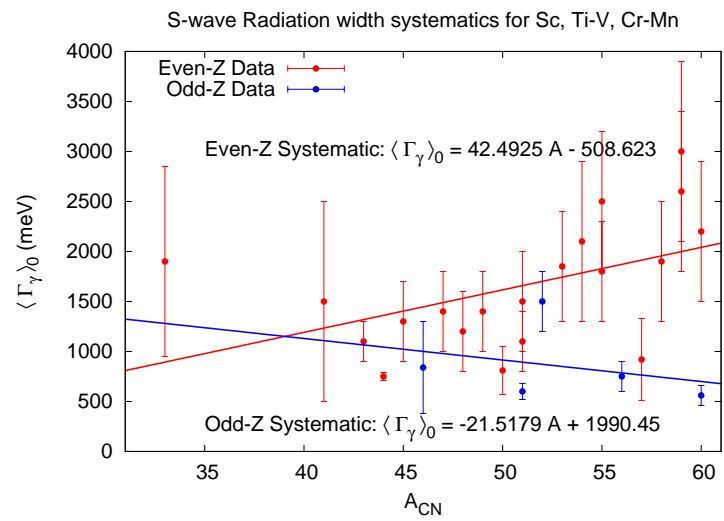

Fig. 3.- Systematics for average total s-wave radiation width. Measured values are from (RIPL 1998), with odd-Z nuclei shown in blue and even- $Z$ nuclei shown in red. The systematics for even- and odd- $Z$ nuclei are indicated by the solid lines, the colors being the same as for the measured data.

(Uhl \& Strohmaier 1976). Here, $J$ is the spin of the target nucleus. The gamma-ray transmission coefficient is evaluated as in Eq. 3. Since the total s-wave radiation width is generally measured only for stable isotopes plus a neutron, we have developed a systematic approach for estimating this value for the many unstable nuclei in our region of interest. The systematic values are determined by a least squares linear fit to experimental data, with a separate systematic for even-Z and odd-Z nuclei (Figure 3). We use the experimental values for all systems that have measured average s-wave radiation widths.

\subsection{Nuclear Level Densities}

\subsubsection{Level Density Models}

Another important input to the statistical model code is the nuclear level density. For this project, we have adopted a standardized, semiempirical approach which is numerically efficient, can be tied to experimental data, and is fairly accurate. The level density is described by two functions. Both are energy dependent, the second factor contains the spin dependence. This is the "Back-shifted Fermi Gas" formulation of the nuclear level density:

$$
\rho(U, J)=\rho(U) f(U, J)
$$

where $\rho(U)$ is the state density, with $U=E-\Delta$ the back-shifted energy. $\Delta$ is the so called "backshift", and $J$ is the spin of the compound nucleus.
We will further treat each component of Eq. 8 in two ways, depending on the excitation energy of interest. The demarcation point will be roughly between the energy range of the known excited levels of a given compound nucleus (the low energy domain), and near (and above) the neutron binding energy (the high energy domain).

For the high energy domain, we describe the level density assuming a Fermi gas formula,

$$
\begin{array}{r}
\rho(U)=\frac{\sqrt{\pi}}{12} \frac{\exp (2 \sqrt{a U})}{a^{1 / 4} U^{5 / 4}} \frac{1}{\sqrt{2 \pi} \sigma} \\
f(U, J)=\frac{2 J+1}{2 \sigma^{2}} \exp \left[\frac{-\left(J+\frac{1}{2}\right)^{2}}{2 \sigma^{2}}\right]
\end{array}
$$

where $a(E)$ is the level density parameter (in $\left.\mathrm{MeV}^{-1}\right)$. The spin cutoff parameter $\sigma^{2}$ is defined as

$$
\sigma^{2}=(0.1223)^{2} A^{5 / 3} \sqrt{\frac{U}{a}}
$$

The level density assumes an equal distribution of parity states. Note that at low excitation energy (for a positive back-shift), Eq. [9] diverges. At low energies, the nuclear level density is better described by a constant temperature formula:

$$
\rho(E) \propto \exp \frac{E-E_{0}}{T}
$$

The level density parameters can be calculated using experimental data. For the Fermi-gas state density (Eq. [9]), the level density parameter, $a(E)$, can be related to the average level spacing $\left(D_{0}\right)$ near the neutron binding energy. The pairing energies used in the calculation of the back shifted energy are calculated as differences of binding energies (Bohr \& Mottelson). The constant temperature parameters $E_{0}$ and $T$, can be chosen to provide a state density that goes through the low lying spectroscopic levels subject to the choice of a matching energy, $E_{x}$, chosen someplace between the high and low energy regions of interest, at which the two state densities match (point and slope). We describe below how we determine these parameters for all of the nuclei considered in this study.

\subsubsection{Level Densities Above the Neutron Bind- ing Energy}

Our goal is to fit the level density parameter $a$ in Eq. [9] to experimental data where available. We adopt an energy dependent form, $a(U, Z, N)$, (Iljinov et al. 1992), and begin by fixing the spin cutoff parameter and the pairing energies. 


\section{The Spin Cutoff Parameter}

The spin cutoff parameter $\sigma^{2}$, Eq. 11, characterizes the spin distribution of the Fermi gas level density. It depends on the level density parameter $a$ of the nucleus in question. In principle it could be determined by experiment, for example, by comparing ratios of cross sections leading to different isomers of the product nucleus (Keisch 1963). Because data like this is often sparse, especially in the limited regions of the periodic chart we are interested in, and because we are often interested in reactions that proceed on or through radioactive species where no such data exists, we must resort to models. In our analysis, we choose the parameterization used by (Rauscher et al. 1997). In previous cross section sets developed for UGT analysis, we used a different parameterization for the spin cutoff parameter for which $\sigma^{2} \propto \sqrt{a U}$. Our justification for using this new parameterization is two-fold. First, it has a sound physical basis and has proven sucessful in other modeling efforts (Rauscher et al. 1997; Woosley et al. 1978). Second, this parameterization helped to refine the systematic behavior of the level density parameter.

\section{Backshift and Shell Corrections}

In determining the back-shift $\Delta$ we adopt the method of (Rauscher et al. 1997). The total backshift is the average of the proton and neutron contributions

$$
\begin{aligned}
\Delta(Z, N) & =\frac{\Delta_{p}+\Delta_{n}}{2} \\
\Delta_{p}(Z, N) & =E^{G}(Z, N) \\
& -\frac{1}{2} E^{G}(Z-1, N) \\
& -\frac{1}{2} E^{G}(Z+1, N) \\
\Delta_{n}(Z, N) & =E^{G}(Z, N) \\
& -\frac{1}{2} E^{G}(Z, N-1) \\
& -\frac{1}{2} E^{G}(Z, N+1)
\end{aligned}
$$

where $E^{G}(Z, N)$ is the binding energy of the nucleus $(Z, N)$. In calculating the binding energies of the various nuclei, we used the experimental mass excesses provided by (Möller et al. 1995). We adopt the so-called "microscopic correction" from (Möller et al. 1995) as our shell corrections, similar to (Rauscher et al. 1997), i.e. $\delta W=E_{m i c .}$.

\section{The Level Density Parameter}

At high energies, the level density parameter $a$ behaves essentially as a function of mass number only. However, in cases where deformation and shell effects are important (often the case near closed neutron shells) it is more appropriate to use an energy dependent form of the level density parameter (Iljinov et al. 1992);

$$
a(U, Z, N)=\tilde{a}(A)\left[1+\delta W(Z, N) \frac{f(U)}{U}\right]
$$

with

$$
f(U)=1-\exp (-\gamma U)
$$

and as usual $U=E-\Delta$. In our analysis, we follow the convention of (Rauscher et al. 1997) in choosing the parameter $\gamma=0.04884$ that describes the fall off in energy of the shell correction.

Classically, the asymptotic level density parameter $\tilde{a}$ is globally fit to a value of $A / 8$. Other attempts at a global fit include (Woosley et al. 1978) which does not use an energy dependent a parameter, and (Rauscher et al. 1997) where $\tilde{a}$ is fit to experimentally determined level densities using a parameterization of $\tilde{a}=\alpha A+\beta A^{2 / 3}$. We continue to follow the methods of the latter.

\subsubsection{Systematic Behavior of Fermi Gas Level Density Parameters}

The asymptotic level density parameter can be determined for select nuclei from experimental values of the average level spacings $D_{0}$ as determined by neutron resonance analysis (RIPL 1998). For $s$-wave resonances (neutron angular momentum equal to zero), the calculated level spacing, $D_{\text {calc }}$, evaluated at the neutron binding energy $U=B_{n}$, is related to the nuclear level density (e.g. Eq.'s [8-10])

$$
D_{\text {calc }}=\frac{2}{\rho\left(U, J=\frac{1}{2}\right)}
$$

for nuclei with spin $s=0$ and

$$
D_{\text {calc }}=\frac{2}{\rho\left(U, J=s+\frac{1}{2}\right)+\rho\left(U, J=s-\frac{1}{2}\right)}
$$

for nuclei with $s \neq 0$. Given our choices for the other parameters affecting the level density $(\lambda, \Delta, \gamma, \delta W)$, we numerically solved for the value of $\tilde{a}$ that would minimize the quantity $\left|D_{\text {calc }}-D_{0}\right|$.

There are only a limited number of nuclei for which the average resonance spacing $D_{0}$ has been measured (i.e. for compound nuclei formed from a 
stable target plus a neutron). As a result, we are required to systematically predict the asymptotic level density parameter for the remaining nuclei in the range of interest for this project. After plotting the "experimental" $\tilde{a}$ in our region of interest and their associated errors, we made a $\chi^{2}$ fit to the data using a parameterization

$$
\tilde{a}=\alpha A+\beta A^{2 / 3}
$$

The fit resulted in the values $\alpha=0.0725442$ and $\beta=0.175418$. These values are markedly different from those of the global fit in (Rauscher et al. 1997). Within the local region of interest, our parameterization results in a smaller average deviation between the systematic and experimentally determined level density parameters, although it would not necessarily provide reasonable values for $\tilde{a}$ for other mass regions.

The derived level density parameters, along with their respective errors, can be seen in Figure 4. The black line represents our local systematic. The blue line represents the global systematic of (Rauscher et al. 1997), and the green line represents the classical $A / 8$ result. Of course, where available, we always use experimentally determined level density parameters over systematic ones.

\subsubsection{Level Densities Below the Neutron Bind- ing Energy}

For the lower energy regions below the neutron binding energy $B_{n}$, the nuclear level density has the same formulation as Eq. [8]. However, particularly at and below the pairing energy $\Delta$, the state density in Eq. [9] becomes imaginary. Unfortunately, experimental level schemes are rarely known above $2 \mathrm{MeV}$ of excitation energy. In practice we are forced again to assume a model and use all available experimental data to constrain its parameters. The two prescriptions for the level density must match tangentially at some energy intermediate to where they are constrained by experiment. Henceforth we will refer to the high energy level density as $\rho_{2}$, and the low energy density as $\rho_{1}$.

Gilbert and Cameron (1965) noticed that the cumulative number of observed levels (the socalled staircase plot, which increases exponentially), can be fit with straight lines in a semi-log plot. They adopted a constant temperature formula to fit these:

$$
N(E) \propto \exp \left[\frac{E-E_{0}}{T}\right]
$$

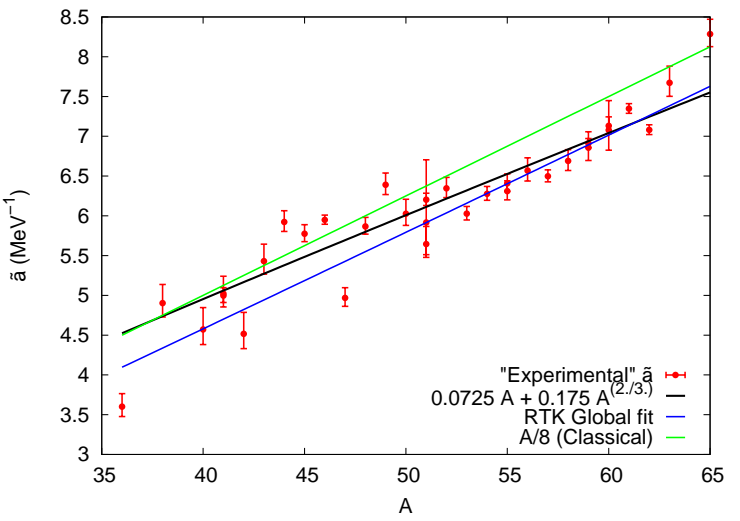

Fig. 4.- $\chi^{2}$ linear fit to derived "experimental" asymptotic level density parameters, used to systematically determine unknown $\tilde{a}$. The measured values, shown in red, are obtained from measured $s$-wave resonance spacings listed in (RIPL 1998), using our chosen parameterizations for the backshift and spin cutoff parameter. Our systematic, fit only to the data shown in this figure, is represented by the solid black line. For reference, the global prescription of (Rauscher et al. 1997) and the classic $\mathrm{A} / 8$ prescription are represented by the solid blue and green lines, respectively.

with $N(E)$ being the cumulative number of levels at excitation energy $E . \quad E_{0}$ and $T$ are two free parameters that may be adjusted to fit the observed level structure. The observable level density is given by

$$
\rho_{1}(E)=\frac{d N(E)}{d E}=\frac{1}{\sqrt{2 \pi} \sigma} \frac{1}{T} \exp \left[\frac{E-E_{0}}{T}\right]
$$

In this local region of interest, we have held the spin cutoff parameter constant below $E_{x}$.

From classical thermodynamics, we have a definition of the nuclear temperature

$$
\frac{d}{d E} \log \rho_{1}(E)=\frac{1}{T}
$$

where $T$ now takes on the meaning of a nuclear temperature which is constant in the region of the discrete levels. We assume that Eq. [19] can be extrapolated from the region of the known discrete levels to higher energies, where the Fermi-gas level density $\left(\rho_{1}\right)$ is valid. We then define the notion of achieving a "good" fit to the total level density over the entire energy range if (a) a good fit can be made to the low lying levels, (b) the observed level spacing at the neutron binding energy is exactly reproduced, (c) the energy of the matching point $E_{x}$ for the two prescriptions falls between $E=\Delta$ 
and $E=B_{n}$, and (d) that they match at this point with the same slope, i.e. for $E=E_{x}$ :

$$
\begin{aligned}
\rho_{1}\left(E_{x}\right) & =\rho_{2}\left(E_{x}\right) \\
\frac{d \log \rho_{1}\left(E_{x}\right)}{d E} & =\frac{d \log \rho_{2}\left(E_{x}\right)}{d E}
\end{aligned}
$$

From Eq. 22, we can determine $E_{0}$ :

$$
E_{0}=E_{x}-T \log T \rho_{2}\left(U_{x}\right)
$$

where $U_{x}=E_{x}-\Delta$. The second condition can be satisfied by assuming that at $E_{x}$ the constant nuclear temperature $T$ of the low lying states is equal to the energy dependent nuclear temperature $\tau\left(U_{x}\right)$ of the high excited states,

$$
\begin{aligned}
\frac{1}{T} & =\sqrt{\frac{a}{U_{x}}}-\frac{3}{2 U_{x}} \\
& +\frac{(\tilde{a}-a)\left(1+\gamma U_{x}\right)+\tilde{a} \gamma \delta W}{\sqrt{a U_{x}}}
\end{aligned}
$$

where $a$ is given by Eq. [14]. If there is no shell correction, the last term in Eq. 25 is zero. Typical values for the matching energy are $2 \leq E_{x} \leq 8$ $\mathrm{MeV}$, and are approximated by $E_{x}=2.5+\frac{150}{A}+\Delta$ (Gilbert \& Cameron 1965). We adjust $E_{x}$ individually for each nucleus to provide the best possible fit to the spectroscopic data. In the event that data is not available for levels above the ground state, the approximation is used. The constant temperature fits to the low lying levels of ${ }^{44} \mathrm{Sc}$, ${ }^{48} \mathrm{~V}$, and ${ }^{52} \mathrm{Mn}$ are presented in Figure 5.

In our attempts to match the the level density to the number of discrete levels, we generally try to ensure that the integrated level density at the energy of the last known level is equal to the cumulative number of known levels, as can be seen in each of the plots in Figure 5. This ensures that the effective level density will be continuous as the transmission coefficients shift between the discrete levels and the level density. However, there are cases where matching at the energy of the last discrete level is not possible (i.e. matching would require $E_{x}<\Delta$ ). Additional restrictions are placed on the matching energy if one requires that the nuclear temperature be positive. Occasionally the resulting lower limit on the matching energy precludes matching the last discrete level, and the integrated level density/cumulative number of levels suffers a discontinuity (recall that the HauserFeshbach formula only employs the level density above the energy of the last discrete level). Such discontinuities result in gross non-physical behavior for some cross sections, particularly $(\mathrm{p}, \mathrm{n})$ cross sections.
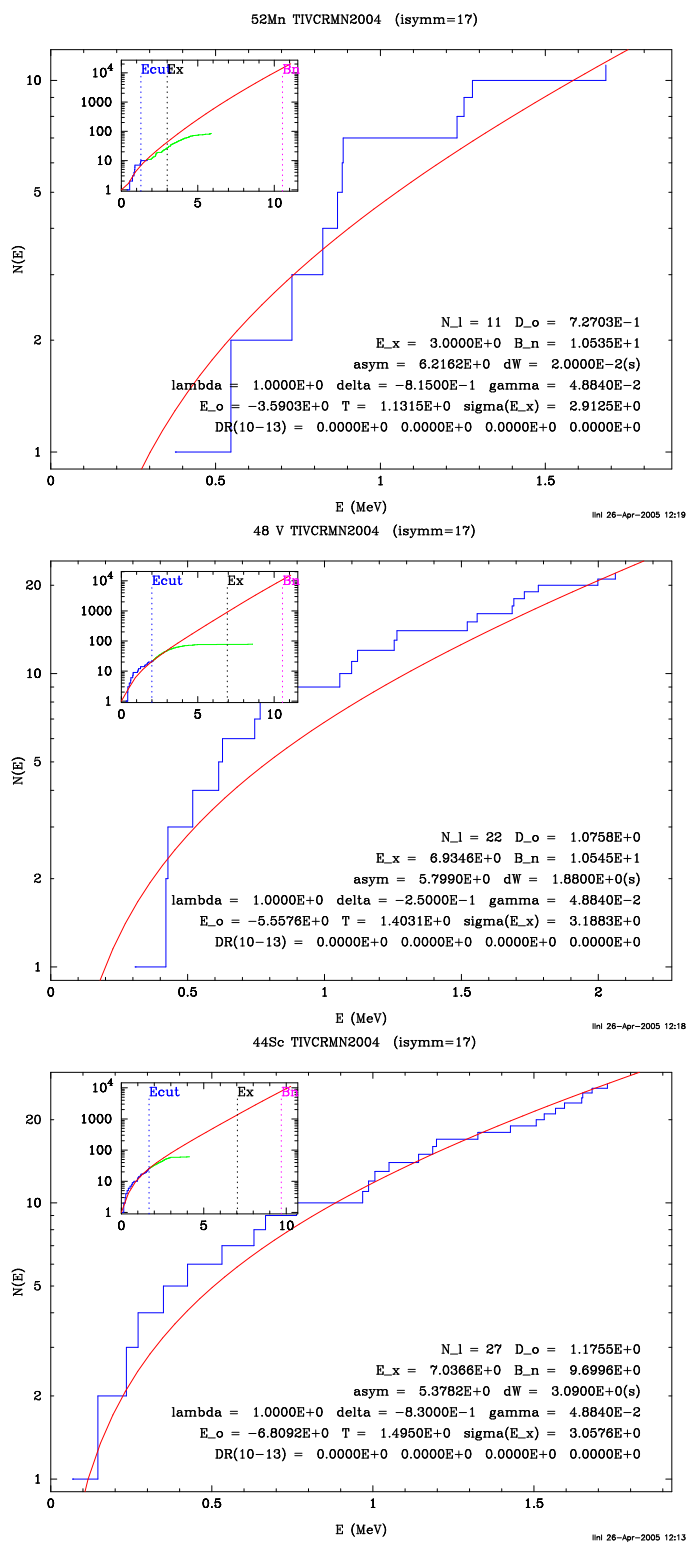

Fig. 5.- Constant temperature level density fits to the low lying spectroscopic levels of ${ }^{44} \mathrm{Sc},{ }^{48} \mathrm{~V}$, and ${ }^{52} \mathrm{Mn}$. The known cumulative number of levels is shown in blue. The green levels, seen in the smaller insets, represent other levels that lie above the energy at which the level scheme is considered complete. The red lines represent the integrated level density.

In most of these cases, one can match the integrated level density to the cumulative number of levels by reducing the number of discrete levels included in the calculation. This is the approach we take. In a few instances, even a reduction in the included discrete levels could not fully rectify the situation. For those nuclei, we use the smallest 
allowed matching energy to reduce the size of the discontinuity as much as possible.

The fitted parameters for the total level density are presented in Table 5 (Appendix 9). The symbols in the legend are the same as described above. In column five, an " $\mathrm{x}$ " indicates the level density parameter $\tilde{a}$ was derived from an experimentally known level spacing $\left(D_{0}\right)$, an "s" indicates that $\tilde{a}$ was derived from the systematic shown in Figure 4 .

\subsection{Considerations Regarding the Exci- ton Model}

When including alpha particles as a possible exit channel, one should account for them in the pre-equilibrium phase of the reaction. Generally, the description of alpha particle emission in the exciton model is a straightforward extension of the description of neutron or proton emission, given the tendency of nucleons to preform alpha clusters in the nucleus. In making such an extension, one introduces a parameter $\phi$ which represents the probability that the incoming particle will strike a preformed alpha cluster (Milazzo-Colli et al. 1973). It follows that the larger values of $\phi$ will result in a higher probability of subsequent alpha emission, thus enhancing the $(\mathrm{n}, \alpha)$ reactions.

In our calculations, we have chosen a value of $\phi=0.50$, although previous considerations of alpha emission suggest that this value may fall anywhere in the range of $0.1 \leq \phi \leq 0.8$ within the mass range of interest (Milazzo-Colli et al. 1973). We have used our chosen value primarily because it results in $(\mathrm{n}, \alpha)$ cross sections which best fit the available experimental cross section data.

Since the alpha particle emission accounts for a relatively small portion of the total reaction cross section (generally less than $20 \%$ ), variations in the $\phi$ parameter will only have minor effects on the other cross sections.

\section{Calculated Cross Sections}

\subsection{Comparison to Measured Cross Sec- tions}

Having developed the various input quantities based on available experimental data in the previous section, we compare cross section results of the STAPRE-H95 model to available experimentally measured cross sections in the region of interest. Only results for targets initially in their ground states are available.
In this section and the sensitivity analysis to follow, we focus on cross sections proceeding on four select target isotopes, ${ }^{45} \mathrm{Sc},{ }^{48} \mathrm{Ti},{ }^{52} \mathrm{Cr}$, and ${ }^{54} \mathrm{Fe}$. Over the last forty years these have been the subject of repeated measurement for all reaction channels relevant to radiochemistry. The latter three are the dominant loaded isotopes leading to production of ${ }^{48} \mathrm{~V}$ in the Ti0886 detector set and ${ }^{52 g} \mathrm{Mn}$ in the Cr0386, and Fe0987 detector sets. Where these four are not available for a given reaction channel we will present comparisons for as many of the loaded titanium detector isotopes as possible. Many other measured cross sections for a variety of reaction channels in this region exist. We provide comparisons to these in Appendix B.

\subsubsection{Neutron capture cross sections}

Figure 6 shows our calculated activation cross section (solid black lines in all plots that follow) defined as the sum of emission (both particle emission and gamma-ray cascade) from the compound nucleus that eventually leads to the ground state of the product (final) nucleus. We also provide (where appropriate) separate cross sections that represent decay to the ground state (red lines), and any long lived isomer (blue lines, see Table 5 in Appendix A for a list of the isomers and their respective half-lives). These cross sections are plotted against the available experimental data, taken from the Experimental Nuclear Reaction Data File (CSISRS 2003). Cross sections for the total, ground, and isomeric states are colored similar the modeled cross sections (grey is activation, magenta is to ground, and cyan to an isomer, respectively), with different symbols distinguishing results from various experiments.

No attempt has been made to evaluate the experimental data. We provide all that is available. This provides a sense for the agreement (or uncertainty) between individual experiments and serves to illustrate the likely error associated with a given cross section. All comparisons made here are repeated in Appendix B. For information on a particular measurement, consult (CSISRS 2003).

We also present in Table 3 a quantitative comparison of our calculated neutron capture activation cross sections to measured $(n, \gamma)$ cross section data at $30 \pm 2 \mathrm{keV}$ of incident energy. For each target listed in column (1), subsequent column entries identify: the number of measured cross section data points in the $30 \pm 2 \mathrm{keV}$ energy range from all available data sets (2), the weighted average of the measured data (in barns) 

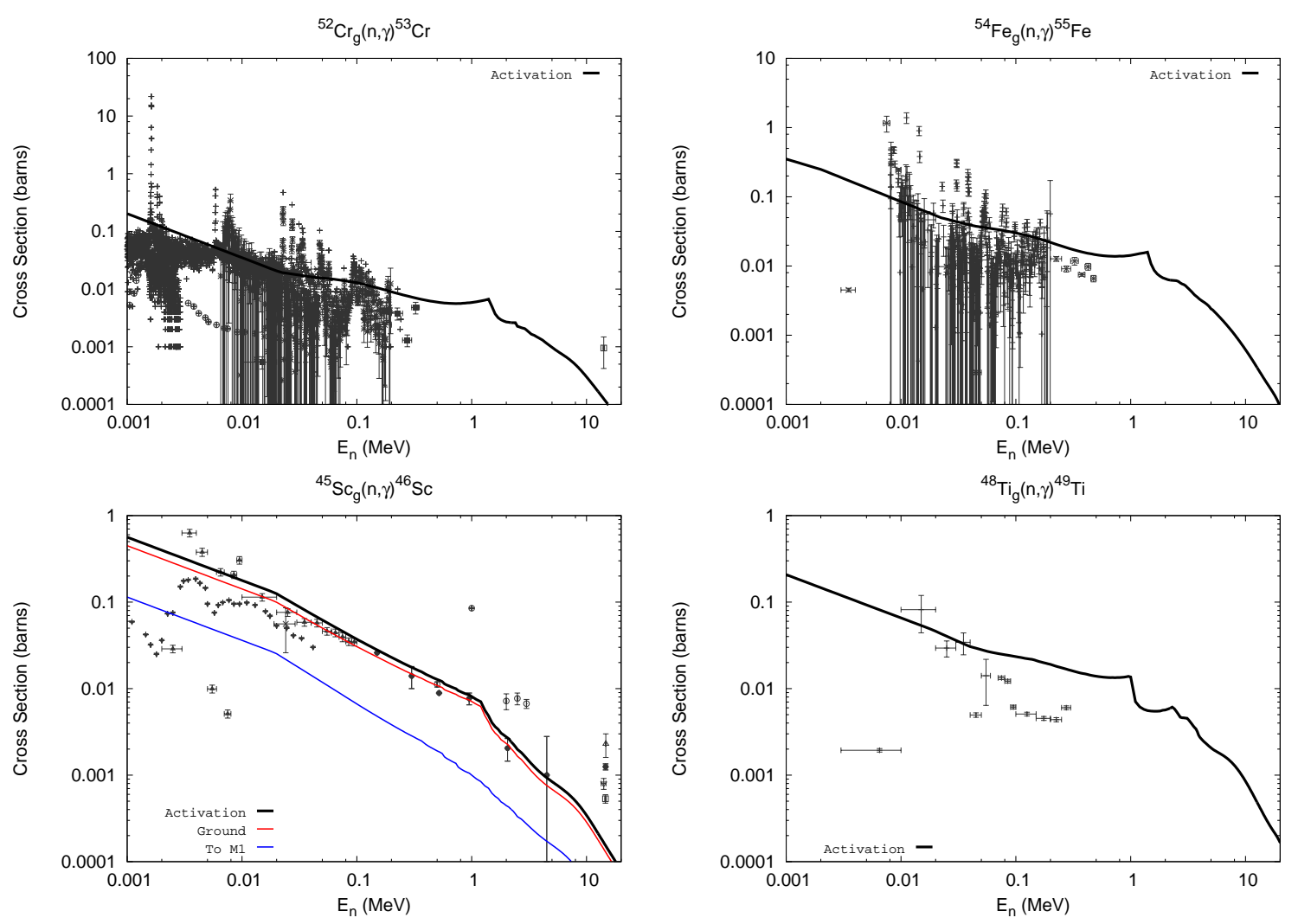

Fig. 6.- Calculated vs. measured (n, $\gamma$ ) cross sections on ${ }^{45} \mathrm{Sc}{ }^{48} \mathrm{Ti},{ }^{52} \mathrm{Cr} \&{ }^{54} \mathrm{Fe}$. Measured cross sections are obtained from (CSISRS 2003). The solid black lines and grey data represent activation cross sections. Solid red lines and magenta data represent the cross section going to the ground state of the residual (equal to the activation cross section when the residual nucleus does not have an isomer). Blue lines and light blue data represent the cross section going to the first isomer.

with weights corresponding to the inverse geometric mean of the errors in cross section and energy (i.e. $\left.w_{i}=\left(d E_{i}^{2}+d \sigma_{i}^{2}\right)^{-1 / 2}\right)(3)$, the standard deviation of the measured data from the weighted average which gives an indication in the spread of the measured data (4), our modeled cross section value at $30 \mathrm{keV}(5)$, and a multiplicative factor that could be applied to our cross section to bring it into conformity with its respective average experimental value. The average error at the bottom of the table is defined as the average percent difference between the weighted average cross sections and our modeled values. This value indicates that, on average, we are within somewhat more than a factor of two of the experimental data.

For the neutron capture cross sections, our result is generally in good agreement with the data between $20-200 \mathrm{keV}$. We do have a tendency to be high by a factor of two to five on even- $\mathrm{N}$ even- $\mathrm{Z}$ targets. Above $200 \mathrm{keV}$, our activation and ground state cross section still show good agreement with the data, with the exception of the data points around $14 \mathrm{MeV}$. It is possible that direct processes are contributing to the cross sections at these energies, and we have not included direct processes in our modeling effort. However, the value of the capture cross section at $14 \mathrm{MeV}$ is only on the order of 10 millibarns, and is insignificant in comparison to other reactions, such as $(n, 2 n)$ and $(n, p)$. Below $10 \mathrm{keV}$, our activation cross section is in good agreement with the data.

Using global systematics, $(n, \gamma)$ cross sections can typically be modeled to within a factor of two, often to within 30\% (Hoffman et al. 1999). In Figure 22 (Appendix B.1), our calculations generally do much better than a factor of two, although there are a few exceptions (particularly ${ }^{50} \mathrm{~V}$ and $\left.{ }^{58} \mathrm{Fe}\right)$. 
Table 3: Comparison of our modeled $(n, \gamma)$ activation cross sections to measured values at $30 \pm 2$ $\mathrm{keV}$. Column one lists the targets for which measured data is available. Column two lists the number of data points that fall in the bin of $30 \pm 2 \mathrm{keV}$. Column three is a weighted average of the data in the bin, in barns, as described in the text. Column four gives the standard deviation of the data points from the weigted average, in barns. Column five lists our calculated values for the neutron capture cross section at $30 \mathrm{keV}$. The last column gives the ratio of the weigted average to our calculated value. The average error shown at the bottom is the average absolute percent difference between our cross sections and the weigted averages.

\begin{tabular}{|c|c|c|c|c|c|}
\hline${ }^{A}{ }^{A} Z$ & $\mathrm{~N}$ & $\overline{\bar{\sigma}}$ & Dev. & $\sigma_{\text {mod }}$ & $\bar{\sigma} / \sigma_{\text {mod }}$ \\
\hline$\frac{{ }^{42} \mathrm{Ca}}{}$ & 1 & 0.018 & 0.000 & 0.034 & 0.513 \\
\hline${ }^{43} \mathrm{Ca}$ & 2 & 0.050 & 0.011 & 0.065 & 0.774 \\
\hline${ }^{44} \mathrm{Ca}$ & 2 & 0.007 & 0.008 & 0.032 & 0.221 \\
\hline${ }^{45} \mathrm{Sc}$ & 4 & 0.059 & 0.012 & 0.091 & 0.643 \\
\hline${ }^{46} \mathrm{Ti}$ & 2 & 0.017 & 0.004 & 0.030 & 0.571 \\
\hline${ }^{47} \mathrm{Ti}$ & 30 & 0.036 & 0.025 & 0.094 & 0.380 \\
\hline${ }^{48} \mathrm{Ti}$ & 2 & 0.031 & 0.002 & 0.036 & 0.871 \\
\hline${ }^{49} \mathrm{Ti}$ & 2 & 0.030 & 0.002 & 0.033 & 0.931 \\
\hline${ }^{50} \mathrm{~V}$ & 1 & 0.015 & 0.000 & 0.041 & 0.366 \\
\hline${ }^{51} \mathrm{~V}$ & 8 & 0.011 & 0.007 & 0.044 & 0.248 \\
\hline${ }^{50} \mathrm{Cr}$ & 54 & 0.014 & 0.024 & 0.050 & 0.279 \\
\hline${ }^{52} \mathrm{Cr}$ & 55 & 0.013 & 0.021 & 0.018 & 0.747 \\
\hline${ }^{53} \mathrm{Cr}$ & 55 & 0.047 & 0.067 & 0.036 & 1.305 \\
\hline${ }^{54} \mathrm{Cr}$ & 26 & 0.023 & 0.008 & 0.019 & 1.260 \\
\hline${ }^{55} \mathrm{Mn}$ & 28 & 0.019 & 0.017 & 0.052 & 0.359 \\
\hline${ }^{54} \mathrm{Fe}$ & 36 & 0.027 & 0.074 & 0.044 & 0.605 \\
\hline${ }^{56} \mathrm{Fe}$ & 63 & 0.019 & 0.019 & 0.018 & 1.007 \\
\hline${ }^{57} \mathrm{Fe}$ & 35 & 0.046 & 0.026 & 0.032 & 1.425 \\
\hline${ }^{58} \mathrm{Fe}$ & 2 & 0.007 & 0.003 & 0.050 & 0.143 \\
\hline \multicolumn{5}{|c|}{ Average error: } & $130.6 \%$ \\
\hline
\end{tabular}

\subsubsection{Maxwellian averaged neutron capture cross sections}

Yet another comparison to experimental data comes from the extensive efforts to evaluate Maxwellian averaged capture cross sections for astrophysical applications (Bao et al. 2000). The Maxwellian-averaged neutron capture cross section is defined as the reaction rate $\langle\sigma v\rangle$ divided by the mean velocity $v_{T}=\sqrt{2 k T / \mu}$ at a given temperature $T$. Here, $\mu$ is the reduced mass. For particle fluences and temperatures typical to stellar nucleosynthesis, the velocity distribution of the neutrons reduces to a Maxwell-Boltzmann distribution. In this case, the Maxwellian-averaged cross section reduces to (Beer et al. 1992)

$$
\begin{aligned}
\frac{\langle\sigma v\rangle}{v_{T}} & =\frac{\int_{0}^{\infty} \sigma_{n \gamma} v \Phi(v) d v}{v_{T}} \\
& =\frac{2}{\sqrt{\pi}(k T)^{2}} \int_{0}^{\infty} \sigma_{n \gamma}(E) W(E, k T) d E
\end{aligned}
$$

where $W(E, k T)=E \exp (-E / k T)$ and $E$ is the center of mass energy.

Figure 7 compares our calculated Maxwellianaveraged capture cross sections to their evaluated counterparts (Bao et al. 2000) for the loaded detector elements. Additional comparisons are given in Appendix B.2. The error bars on all points are identical and represent the measured error for a given cross section at $30 \mathrm{keV}$. We use spline interpolation to determine the value of the $(\mathrm{n}, \gamma)$ cross section between points on the energy grid. For energies below our lowest grid energy, we assume an $(\mathrm{n}, \gamma)$ cross section with an $E_{l a b}^{-1 / 2}$ dependence. For energies greater than our highest grid energy, we take the cross section to be zero.

Overall our calculated maxwellian averaged cross sections agree with those of (Bao et al. 2000). Our results for a few targets tend to be high, but in a manner consistent with our comparison to other $(\mathrm{n}, \gamma)$ cross sections. It should be kept in mind that for comparisons to neutron induced experimental capture cross sections we are considering compound nuclear systems for which the important input parameters to our reaction model (e.g. those that affect level densities and photontransmission coefficients) are often determined by normalization to experimental data (e.g. from resonance analysis), and so one would expect the comparisons to be good. Since these compound nuclei often bracket the systems of most interest to us, namely those which account for the dominant destruction reactions like $(\mathrm{n}, \gamma)$, our systematics should reasonably provide for similar agreement.

\subsection{3. (n,2n) cross sections}

Among the more important neutron induced reactions, particularly above $10 \mathrm{MeV}$, are the $(\mathrm{n}, 2 \mathrm{n})$ reactions. In Figure 8 we present our calculated $(\mathrm{n}, 2 \mathrm{n})$ cross sections on the loaded detector elements. For ${ }^{45} \mathrm{Sc}$ and ${ }^{54} \mathrm{Fe}$, the agreement with the measured activation cross section is very good. We note that the ${ }^{54} \mathrm{Fe}(\mathrm{n}, 2 \mathrm{n}){ }^{53} \mathrm{Fe}$ cross section includes measured values for the amount going to the ground state and isomer. Because the energy of the isomer in ${ }^{53} \mathrm{Fe}(3.04 \mathrm{MeV})$ lies well above the energy for which the discrete level scheme is 

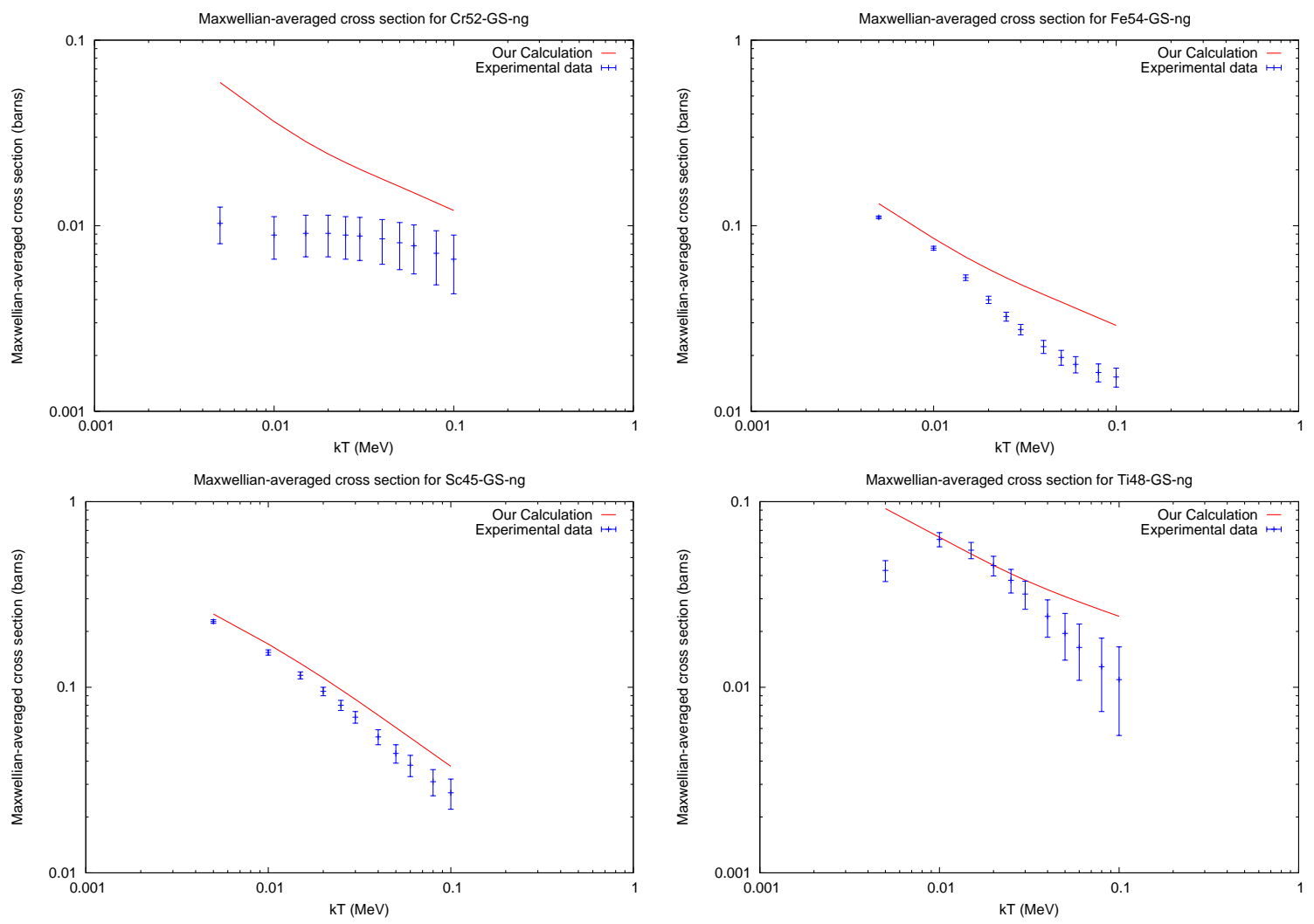

Fig. 7.- Calculated vs. recommended Maxwellian averaged (n, $\gamma)$ cross sections on ${ }^{45} \mathrm{Sc},{ }^{48} \mathrm{Ti},{ }^{52} \mathrm{Cr}, \&{ }^{54} \mathrm{Fe}$. The recommended values, shown in blue, are obtained from (Bao et al. 2000). Our calculated values are indicated by the solid red lines.

complete $(1.70 \mathrm{MeV})$, we were unable to calculate its value in our modeling effort. For ${ }^{52} \mathrm{Cr}$ we slightly overestimate the cross section at peak, although our calculation is easily within $10 \%$. For the ${ }^{48} \mathrm{Ti}(\mathrm{n}, 2 \mathrm{n})$ cross section, (CSISRS 2003) cites only a single measurement at $14.7 \mathrm{MeV}$ with a value of 0.039 barns. This is in major conflict with not only our calculation but also with nuclear theory. As a rule of thumb $(\mathrm{n}, 2 \mathrm{n})$ cross sections scale roughly as the size of the nucles and at $14 \mathrm{MeV}$ a value between $0.5-2$ barns is nearly universal in this mass range. Here we have three exceptions, ${ }^{54} \mathrm{Fe}$, a closed shell nucleus, and ${ }^{46} \mathrm{Ti}$ and ${ }^{50} \mathrm{Cr}$, both with $(\mathrm{n}, 2 \mathrm{n})$ thresholds near $13 \mathrm{MeV}$.

A recent experiment carried out under the Academic Alliance for Stockpile Stewardship Program (Dugersuren et al. 2005) studied spallation neutrons incident on isotopically pure ${ }^{48} \mathrm{Ti}$, measuring $\gamma$-ray excitation functions which were converted to partial $\gamma$-ray cross sections. Coupled with the statistical modeling results from this effort, total cross sections for various neutron induced reactions were derived. The ${ }^{48} \mathrm{Ti}(\mathrm{n}, 2 \mathrm{n})^{47} \mathrm{Ti}$ reaction is shown in Figure 9, giving the observed $(\mathrm{n}, 2 \mathrm{n})$ cross section from the gamma-ray spectra (in red), and the corrected (total) cross section (in black), where the amount of the cross section due to partial $\gamma$-rays that were not observed was provided by the STAPRE gamma-ray cascade calculation. The agreement is excellent.

Additional comparisons to experimental data may be found in Appendix B.3, where our results show similar agreement. Three exceptions worth noting are ${ }^{55} \mathrm{Mn}$ and ${ }^{56} \mathrm{Fe}$, both of which show conflicting data with our calculation in agreement with the more common data points, and ${ }^{50} \mathrm{Cr}$ which we are high by a factor of two.

A quantitative comparison between our modeling effort and measured $(\mathrm{n}, 2 \mathrm{n})$ cross section data is provided in Table 4 . The format of this table is identical to that of Table 3. The average error is highly affected by the suspect ${ }^{48} \mathrm{Ti}(\mathrm{n}, 2 \mathrm{n})$ measurement and those with thresholds near 14 $\mathrm{MeV}$. When these are excluded, the averge error is $12.8 \%$. 

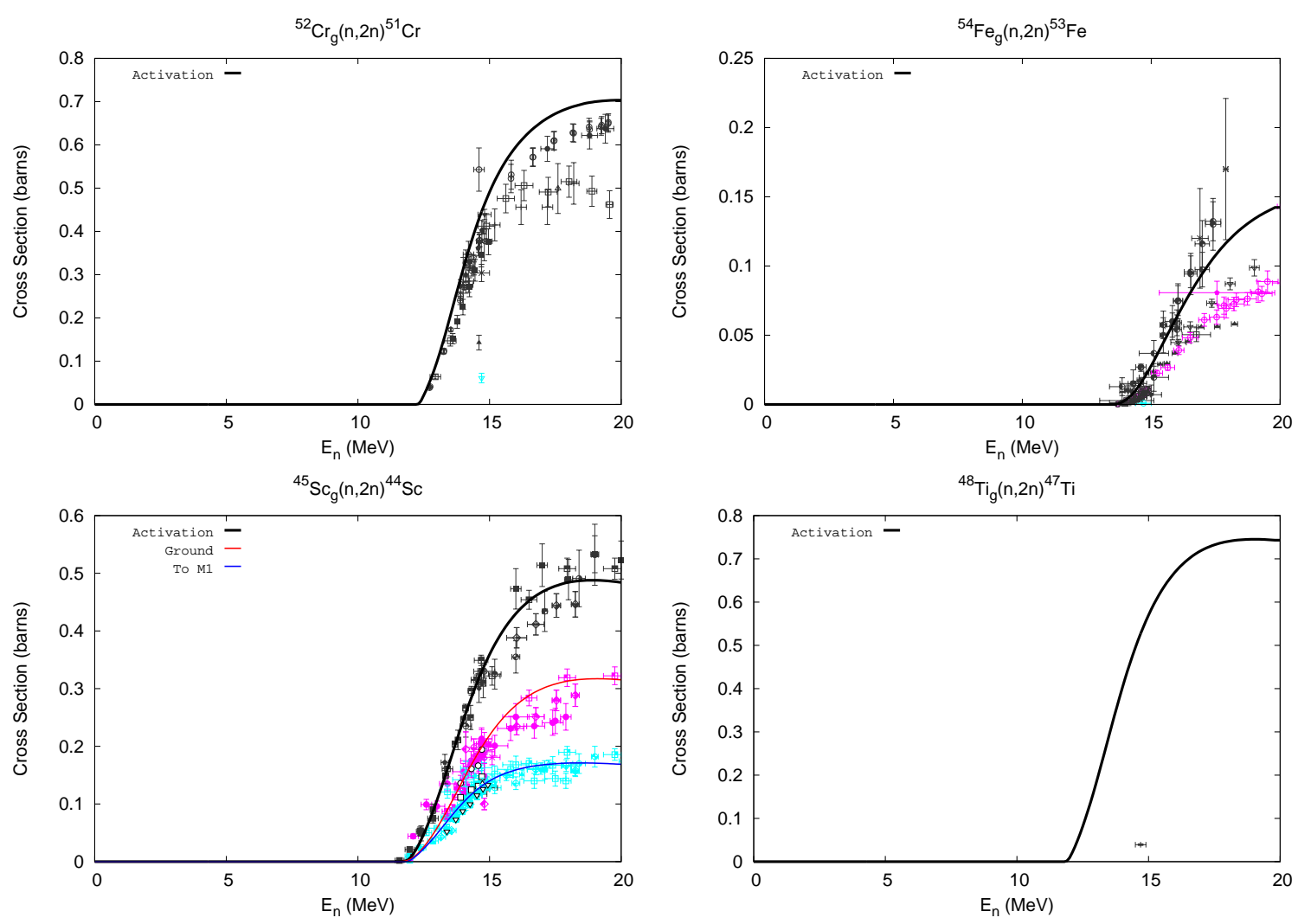

Fig. 8. - Calculated vs. measured (n,2n) cross sections on ${ }^{45} \mathrm{Sc},{ }^{48} \mathrm{Ti},{ }^{52} \mathrm{Cr}, \&{ }^{54} \mathrm{Fe}$. Measured cross sections are taken from (CSISRS 2003). The solid black lines and grey data represent activation cross sections. Solid red lines and magenta data represent the cross section going to the ground state of the residual (equal to the activation cross section when the residual nucleus does not have an isomer). Blue lines and light blue data represent the cross section going to the first isomer.

\subsubsection{Neutron induced charged particle exit channel cross sections}

The remaining neutron induced reactions which are important in the local region of interest include charged particles in the exit channels. In particular, the (n,p), (n,np), and (n,pn) reactions are somewhat significant, and in some cases may be larger than the $(\mathrm{n}, 2 \mathrm{n})$ cross section at 14 $\mathrm{MeV}$. This is particularly true for the lighter, more proton-rich species of each element.

Figure 10 compares our modeled (n,p) cross sections against experimental data for the select loaded detector isotopes, with additional comparisons in Figure 25 (Appendix B.4).

Our results for the $(\mathrm{n}, \mathrm{p})$ cross sections are mixed. There are cases where we are low (e.g. $\left.{ }^{46} \mathrm{Ti}\right)$, and cases where we are high (e.g. $\left.{ }^{45} \mathrm{Sc}\right)$. There are also cases where we our calculations are in good overall agreement with the data, such as ${ }^{48} \mathrm{Ti}$. The (n,p) cross sections presented in the Ap- pendix show a similar agreement with the data. Generally, our calculation is within $30 \%$ at the peak. In all cases, we are at least roughly within a factor of two.

There are only limited amounts of $(\mathrm{n}, \mathrm{np})$ and $(\mathrm{n}, \mathrm{pn})$ data available in this region. For the loaded detector elements, experimental data is only available for three of the titanium isotopes. We present in Figure 11 the combined (n,np) and (n,pn) cross sections on these isotopes. Additional comparisons may be found in Appendix B.5. Our overall agreement with the limited data available for these reactions is good.

Additionally, we have calculated $(\mathrm{n}, \alpha)$ and $(\mathrm{n}, \mathrm{d})$ reactions on all target nuclei. These cross sections tend to be significantly smaller than the dominant reaction channels, and so should not play as an important role in the radiochemical UGT analysis. Comparisons to available experimental data are provided in Appendix B.6 and B.7. 

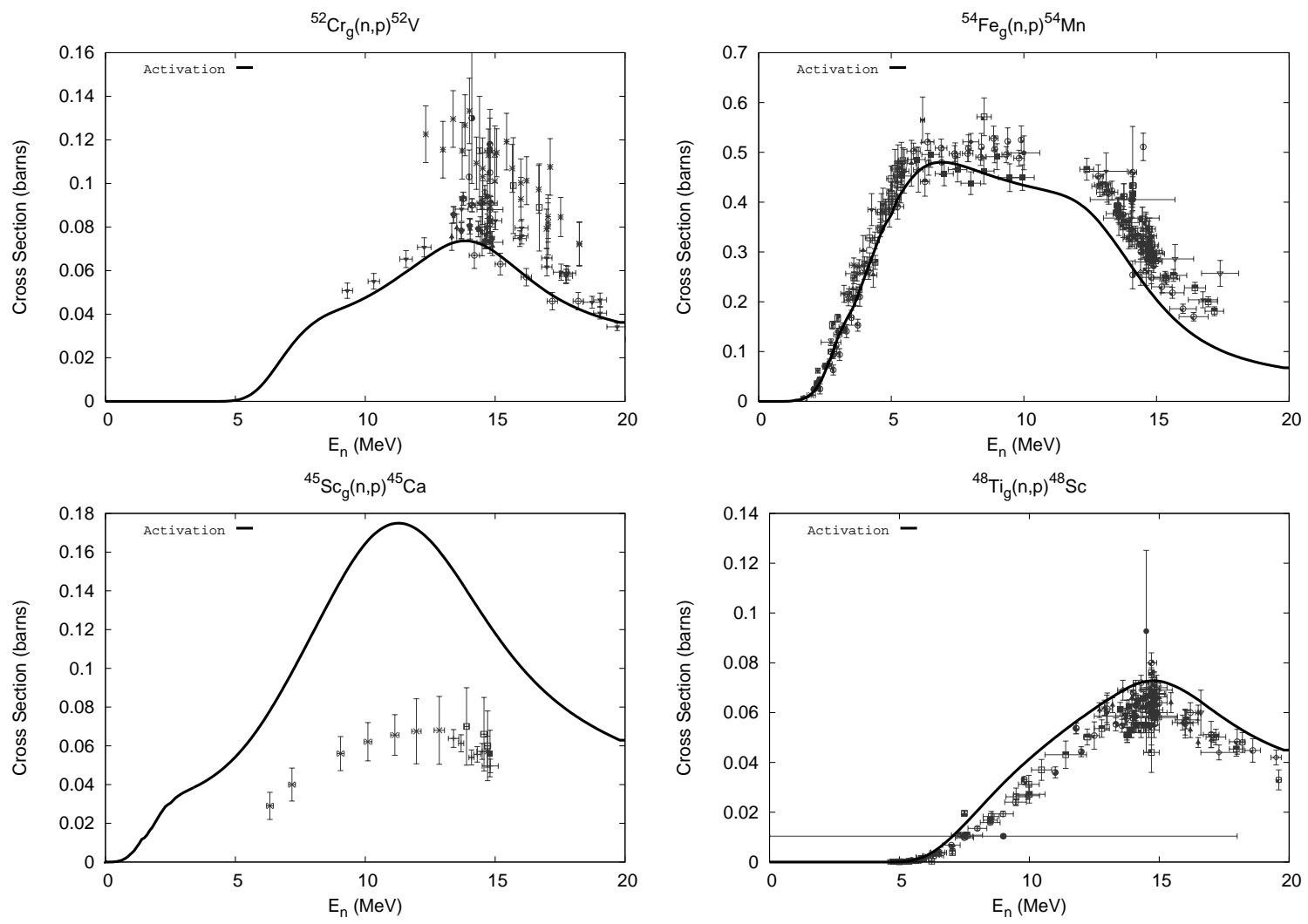

Fig. 10.- Calculated vs. measured (n,p) cross sections on ${ }^{45} \mathrm{Sc},{ }^{48} \mathrm{Ti},{ }^{52} \mathrm{Cr}, \&{ }^{54} \mathrm{Fe}$. Measured cross sections are obtained from (CSISRS 2003). The solid black lines and grey data represent activation cross sections.

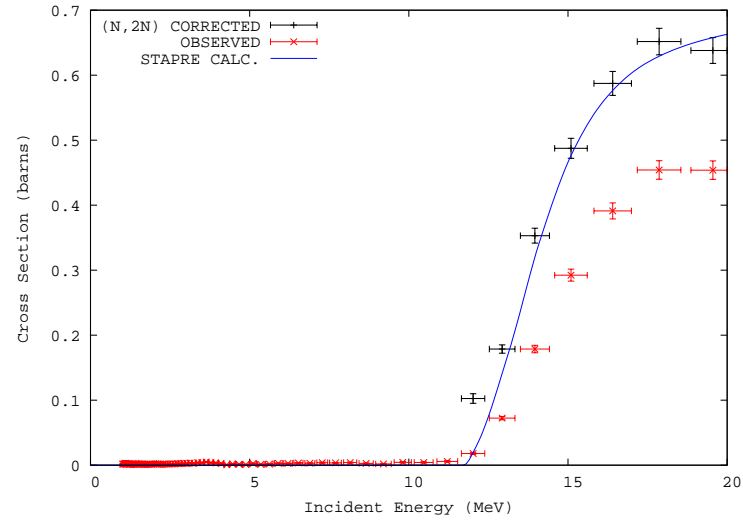

Fig. 9.- Observed and corrected ${ }^{48} \mathrm{Ti}(\mathrm{n}, 2 \mathrm{n})^{47} \mathrm{Ti}$ cross sections from (Dugersuren et al. 2005). The observed $(\mathrm{n}, 2 \mathrm{n})$ cross section is shown in red, and the corrected values (as described in the text) are shown in black. Our activation cross section is represented by the solid blue line. All further ${ }^{48} \mathrm{Ti}(\mathrm{n}, 2 \mathrm{n})$ comparisons will be to this result.

We have also calculated (n,n') reactions for all targets with long-lived isomers and compound
Table 4: Comparison of modeled $(\mathrm{n}, 2 \mathrm{n})$ activation cross sections to measured values at $14.7 \pm 0.2$ $\mathrm{MeV}$. Columns are the same as in Table 3. Two error estimates are given, the latter excludes three nuclei with thresholds near $14 \mathrm{MeV}$ and one whose measured value is unphysical. See text.

\begin{tabular}{clllll}
\hline \hline${ }^{A} Z$ & $\mathrm{~N}$ & $\bar{\sigma}$ & Dev. & $\sigma_{\text {mod. }}$ & $\bar{\sigma} / \sigma_{\text {mod. }}$ \\
\hline${ }^{48} \mathrm{Ca}$ & 5 & 0.675 & 0.152 & 0.771 & 0.875 \\
${ }^{45} \mathrm{Sc}$ & 10 & 0.299 & 0.082 & 0.329 & 0.908 \\
${ }^{46} \mathrm{Ti}$ & 19 & 0.043 & 0.011 & 0.067 & 0.651 \\
${ }^{48} \mathrm{Ti}$ & 1 & 0.039 & 0.000 & 0.527 & 0.074 \\
${ }^{51} \mathrm{~V}$ & 1 & 0.660 & 0.000 & 0.614 & 1.076 \\
${ }^{50} \mathrm{Cr}$ & 21 & 0.024 & 0.005 & 0.056 & 0.429 \\
${ }^{52} \mathrm{Cr}$ & 13 & 0.350 & 0.089 & 0.446 & 0.785 \\
${ }^{55} \mathrm{Mn}$ & 35 & 0.779 & 0.088 & 0.799 & 0.976 \\
${ }^{54} \mathrm{Fe}$ & 25 & 0.011 & 0.009 & 0.018 & 0.573 \\
${ }^{56} \mathrm{Fe}$ & 5 & 0.470 & 0.128 & 0.542 & 0.867 \\
\hline \multicolumn{5}{c}{ Average error: } & $158.3 \%$ \\
\multicolumn{5}{c}{ (excluding ${ }^{46,48} \mathrm{Ti},{ }^{50} \mathrm{Cr}, \&$ 年 $\left.\mathrm{Fe}\right):$} & $12.8 \%$ \\
\hline \multicolumn{5}{c}{}
\end{tabular}

elastic cross sections for all targets. Limited experimental data is available for comparison with the 

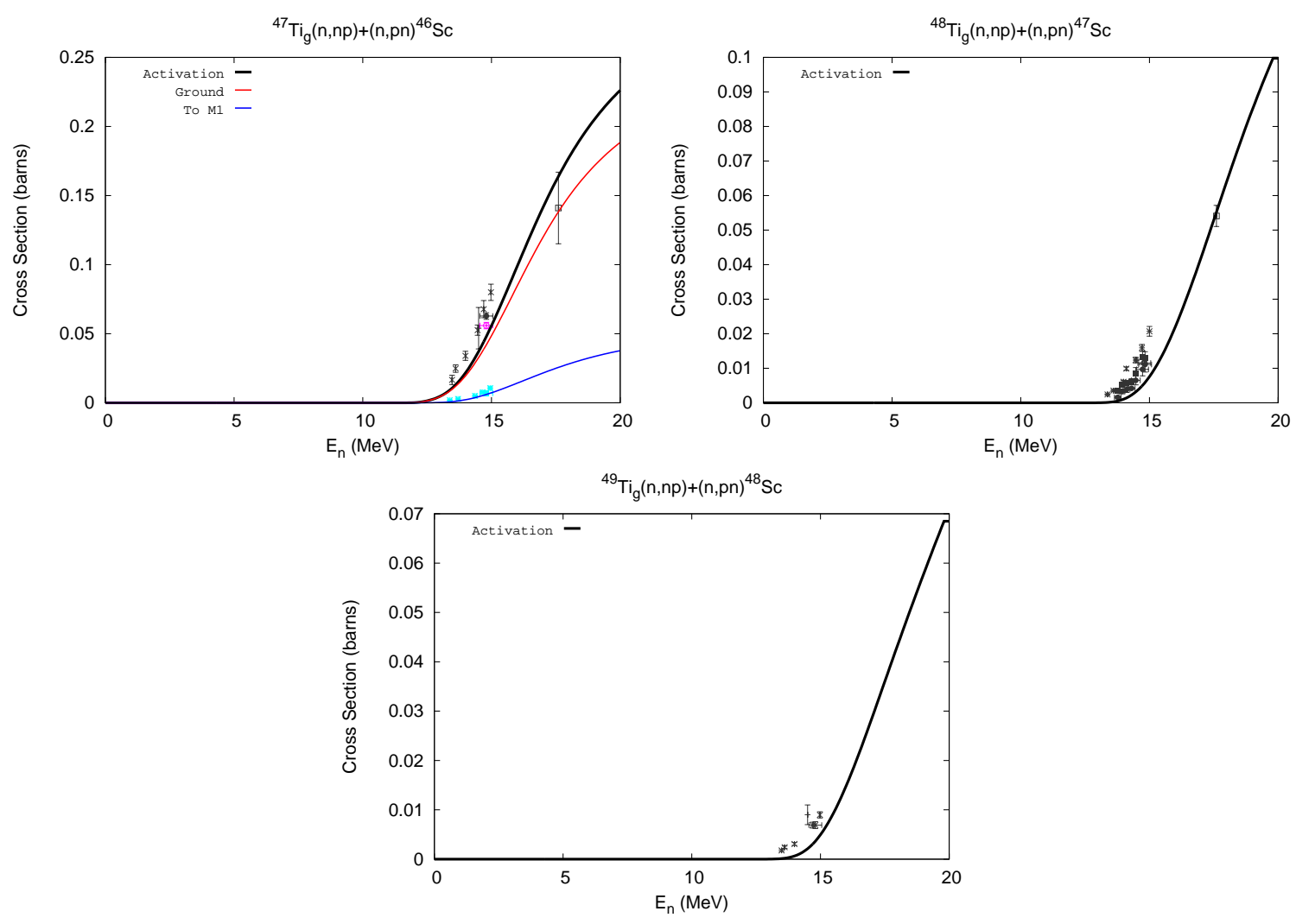

Fig. 11.- Calculated vs. measured $(\mathrm{n}, \mathrm{np})+(\mathrm{n}, \mathrm{pn})$ cross sections on ${ }^{47-49} \mathrm{Ti}$. Measured cross sections are obtained from (CSISRS 2003). The solid black lines and grey data represent activation cross sections. Solid red lines and magenta data represent the cross section going to the ground state of the residual (equal to the activation cross section when the residual nucleus does not have an isomer). Blue lines and light blue data represent the cross section going to the first isomer.

$(\mathrm{n}, \mathrm{n}$ ') calculations, with all available data corresponding to inelastic ground state to ground state reactions. Comparsions to the data may be seen in Appendix B.8.

\subsubsection{Proton-induced cross sections}

In Figure 12 we show our modeled cross section for ${ }^{47} \mathrm{Ti}(\mathrm{p}, \mathrm{n})^{47} \mathrm{~V},{ }^{48} \mathrm{Ti}(\mathrm{p}, \mathrm{n})^{48} \mathrm{~V},{ }^{49} \mathrm{Ti}(\mathrm{p}, \mathrm{n})^{49} \mathrm{~V}$, and ${ }^{52} \mathrm{Cr}(\mathrm{p}, \mathrm{n})^{52} \mathrm{Mn}$ compared to experimental data. Normally one would expect a $(\mathrm{p}, \mathrm{n})$ cross section to rise smoothly from threshold to the peak. Many of our (p,n) cross sections exhibit additional structure in the rise from threshold. This behavior is due primarily to the discrete level spectrum provided as input to the Hauser-Feshbach formula (Eq. 1). In particular, these effects arise from the spacing of the discrete levels, which can be relatively large (as much as a few $\mathrm{MeV}$ ) in the lower mass regions of the periodic chart, and particularly large for closed shell nuclei.

Appendix B.9 shows additional comparisons of $(\mathrm{p}, \mathrm{n})$ cross sections to experimental data. The overall behavior is similar to that seen in Figure 12. Our modeling effort also includes $(\mathrm{p}, 2 \mathrm{n})$ reactions for which only limited data is available.

\subsubsection{Deuteron-induced cross sections}

We have modeled deuteron induced cross sections, including both $(\mathrm{d}, \mathrm{n})$ and $(\mathrm{d}, 2 \mathrm{n})$ reactions. When modeling these cross sections, one must account for deuteron breakup in the presence of the Coulomb barrier (Udagawa \& Tamura 1986). Since STAPRE-H95 does not include such models, we operationally account for them by a suitable reduction of the deuteron total reaction cross section (Mustafa 1997). For each target we calculate the breakup and breakup-fusion cross sections for incident deuterons. Separate calculations are performed for the fusion of the neutron to the target and the fusion of the proton to the target. These cross sections are then summed to find the total deuteron breakup-fusion cross section, expressed 

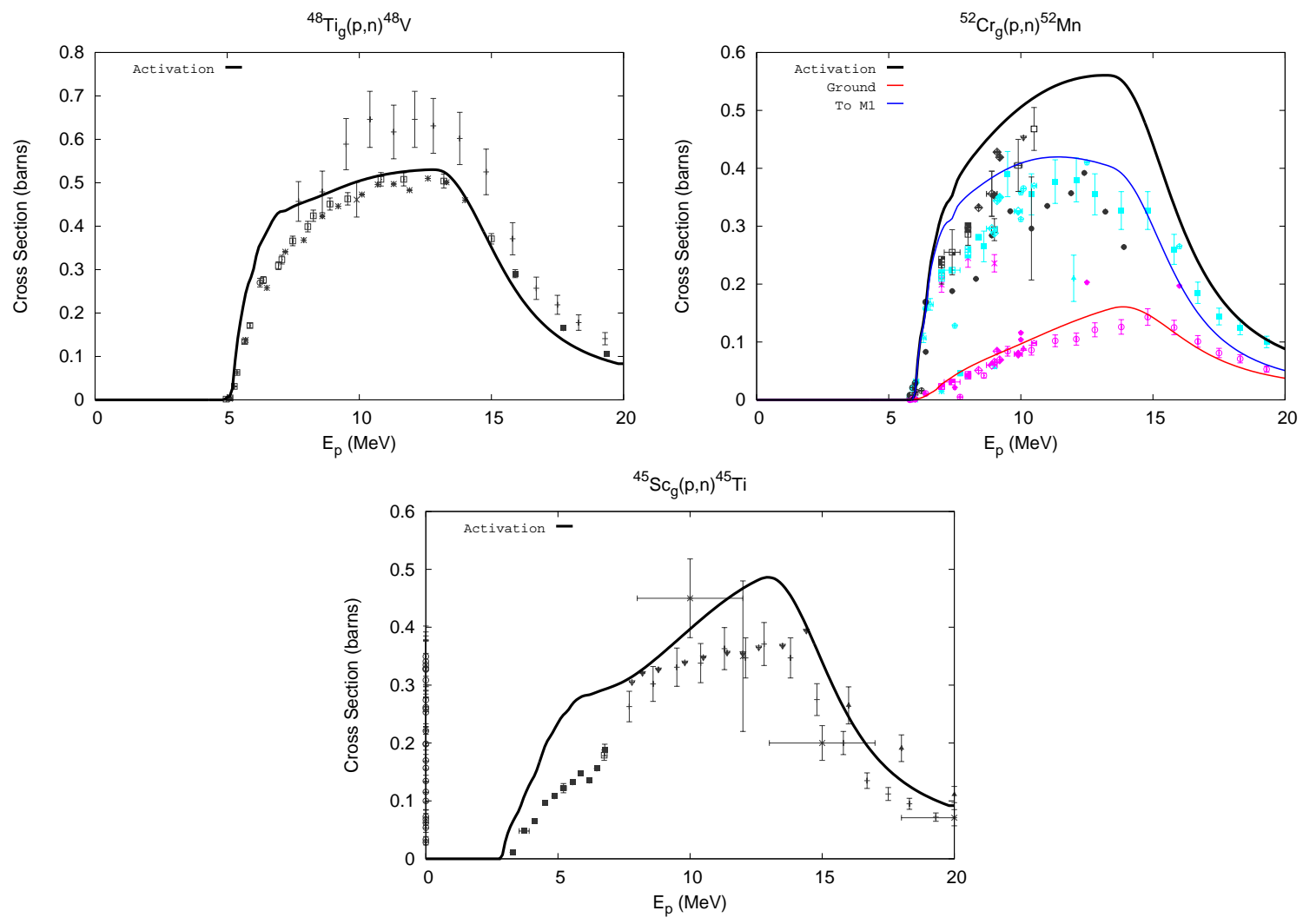

Fig. 12.- Calculated vs. measured (p,n) cross sections on ${ }^{45} \mathrm{Sc},{ }^{48} \mathrm{Ti}, \&{ }^{52} \mathrm{Cr}$. Measured cross sections are obtained from (CSISRS 2003). The solid black lines and grey data represent activation cross sections. Solid red lines and magenta data represent the cross section going to the ground state of the residual (equal to the activation cross section when the residual nucleus does not have an isomer). Blue lines and light blue data represent the cross section going to the first isomer.

as a fraction of the total reaction cross section at each incident energy. We then reduce the reaction cross section in the Hauser-Feshbach calculation accordingly. Select sample breakup fractions are shown in Figure 13.

The deuteron breakup calculations include the fusion of one of the incident nucleons to the target. This process is similar to pre-equilibrium emission of a nucleon. For example, the breakup-fusion of the proton to the target mimics the absorption of the deuteron followed by the pre-equilibrium emission of a neutron. To avoid "double counting" such processes, we do not include pre-equilibrium in our deuteron induced cross sections.

In Figure 14 we compare our modeled deuteron induced cross sections with available measured data. Additional comparisons are given in Appendices B.11 and B.12. Our modeled (d,n) cross sections generally compare favorably with measured data for the first few $\mathrm{MeV}$ above threshold. However, our cross sections are consistently

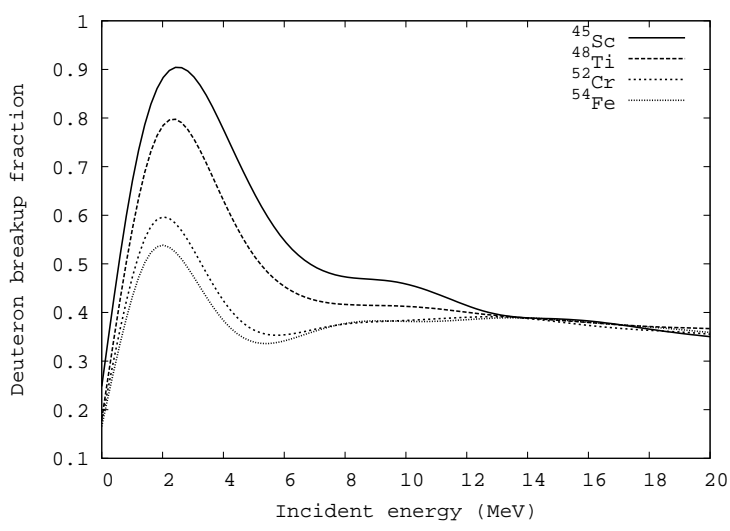

Fig. 13.- Sample deuteron breakup fractions for ${ }^{45} \mathrm{Sc},{ }^{48} \mathrm{Ti},{ }^{52} \mathrm{Cr}$, and ${ }^{54} \mathrm{Fe}$

low by $50-100 \mathrm{mb}$ beyond the peak. This is most likely due to an incomplete consideration of the deuteron breakup processes. In particular, the breakup-fusion process that results in the fusion 

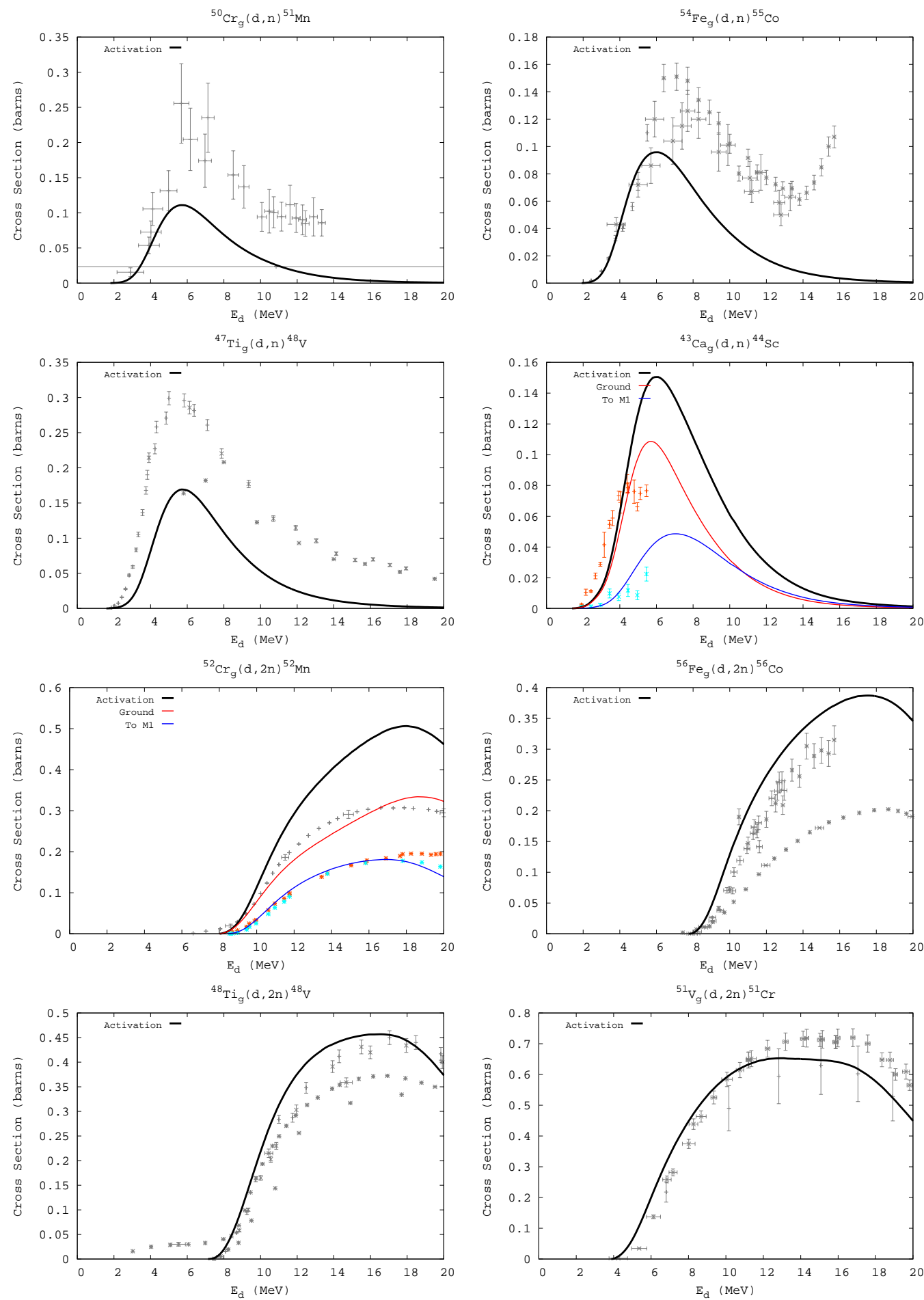

Fig. 14.- Calculated vs. measured $(\mathrm{d}, \mathrm{n})$ and $(\mathrm{d}, 2 \mathrm{n})$ cross sections. Measured cross sections are obtained from (CSISRS 2003). The solid black lines and grey data represent activation cross sections. In cases where the residual has an isomer, red lines and data represent the cross section going to the ground state and blue lines and data represent the cross section going to the first isomer.

of the incident proton to the target populates the excited states of the residual of the $(\mathrm{d}, \mathrm{n})$ reaction with a cross section of $\sim 250 \mathrm{mb}$ above $6 \mathrm{MeV}$ of incident energy. The resulting compound nucleus 
must then de-excite by the emission of radiation or particles. Hence, some of this breakup-fusion cross section should be added back to the modeled $(d, n)$ cross section, though it is not entirely clear how much. Initially one might assume that the decay of the compound nucleus after breakupfusion would be similar to the decay of the same compound nucleus formed by the absorption of the proton, although it is not clear whether the same excited states would be populated in each case. This issue remains the subject of ongoing study.

For the $(\mathrm{d}, 2 \mathrm{n})$ cross sections, the comparison with measured data is generally favorable. Th only notable exception is that we have overestimated the ${ }^{52} \mathrm{Cr}(\mathrm{d}, 2 \mathrm{n})^{52} \mathrm{Mn}$ cross section going to the ground state of ${ }^{52} \mathrm{Mn}$ by $\sim 60 \%$, though our agreement with the isomer cross section is very good. These two measurements are from (West et al. 1993). Three of the activation cross section measurements (specifically the lower cross sections on ${ }^{48} \mathrm{Ti}$ and ${ }^{56} \mathrm{Fe}$ and activation cross section for ${ }^{52} \mathrm{Cr}$ ) are from the same author (Burgus et al. 1954) and are consistently lower than recent measurements. Excluding these three measurements, our modeled $(\mathrm{d}, 2 \mathrm{n})$ activation cross sections fall within $15 \%$ of the measured data. Small amounts of measured cross section data were available for ${ }^{48} \mathrm{Ca}$ and ${ }^{50} \mathrm{Cr}$ targets. Comparisons for these reactions are found in Appendix B.12. We note that the higher of the measurements for ${ }^{48} \mathrm{Ti}$ is from (West et al. 1993).

The most important charged particle reactions relevent to UGT analysis have been experimentally measured, and will be used in lieu of modeled cross sections in the classified UGT analysis. Other charged particle reactions may play a role in the production of species of interest, although such production would necessarily proceed through a series of reactions. The total production of the radioisotopes of interest through these secondary channels will be small compared to the direct production via charged particle interactions on the loaded detector elements. Consequently, errors in the modeled charged particle cross sections should have minimal if not negligible effects.

\subsection{Sensitivity Studies}

We illustrate the sensitivity of our modeled results to variations in the input parameters developed in $\S 3$ against the measured activation cross sections for $(n, \gamma),(n, 2 n),(n, p)$ and $(p, n)$ on ${ }^{45} \mathrm{Sc}$, ${ }^{48} \mathrm{Ti}$, and ${ }^{52} \mathrm{Cr}$.

\subsubsection{Sensitivity to the Pre-Equilibrium Cross Section}

We adopt a simple exciton model with initial 2-particle 1-hole configuration. Average rates for internal transitions are related by the formulas of Williams (1970), corrected for the Pauli principle by Cline (1972), to the absolute square of the average effective matrix element $|M|$ of the residual interactions as per Eq. (7) of (Uhl \& Strohmaier 1976). The dependence of $|M|^{2}$ on mass number and excitation energy is

$$
|M|^{2}=\langle F M\rangle A^{-3} E^{-1}
$$

Figures (15-16) show the sensitivity of the (n,2n), $(\mathrm{n}, \mathrm{p})$, and $(\mathrm{p}, \mathrm{n})$ cross sections on ${ }^{45} \mathrm{Sc},{ }^{48} \mathrm{Ti}$, and ${ }^{52} \mathrm{Cr}$ targets for variations of $100 \leq\langle F M\rangle \leq 400$.

The effect on the $(\mathrm{n}, 2 \mathrm{n})$ cross sections in Figure 15 is that larger values of $\langle F M\rangle$ result in larger cross sections. An opposite effect appears in the (n,p) cross sections (16), with larger values of $\langle F M\rangle$ tending to result in smaller cross sections at higher energies. However, at energies below 14 $\mathrm{MeV}$ no consistent trends are apparent. For the $(\mathrm{p}, \mathrm{n})$ cross sections, larger values of $\langle F M\rangle$ tend to increase the cross section at peak and decrease the cross section in the tail. Pre-equilibrium plays a negligible role at low energies where neutron capture cross sections are large, and hence the neutron capture cross sections do not exhibit any sensitivity to the value of $\langle F M\rangle$.

We evaluated every cross section for which there is experimental data in the region of interest using values of $\langle F M\rangle=100,200$, and 400. Placing a greater weight on the cross sections known to be larger over the energy ranges of interest, we found that a value of $\langle F M\rangle=200$ provided the best overall agreement with experimental data. This value for $\langle F M\rangle$ was applied in calculating the preequilibrium contribution to all cross sections in this study.

\subsubsection{Sensitivity to the choice of Level Density Prescription}

The nuclear level density parameters developed for this study (Appendix 9) reflect best choices from the available experimental data in the region of interest. Instead of varying each of the many parameters $\left(a(U, Z, N), \sigma^{2}, \lambda, \Delta, \delta_{W}\right)$, we instead present results where only the overall treatment of the level density prescription was varied, keeping all other parameter input fixed.

Figures (17-18) show the experimental cross sections for $(n, \gamma),(n, 2 n),(n, p)$, and $(p, n)$ cross 

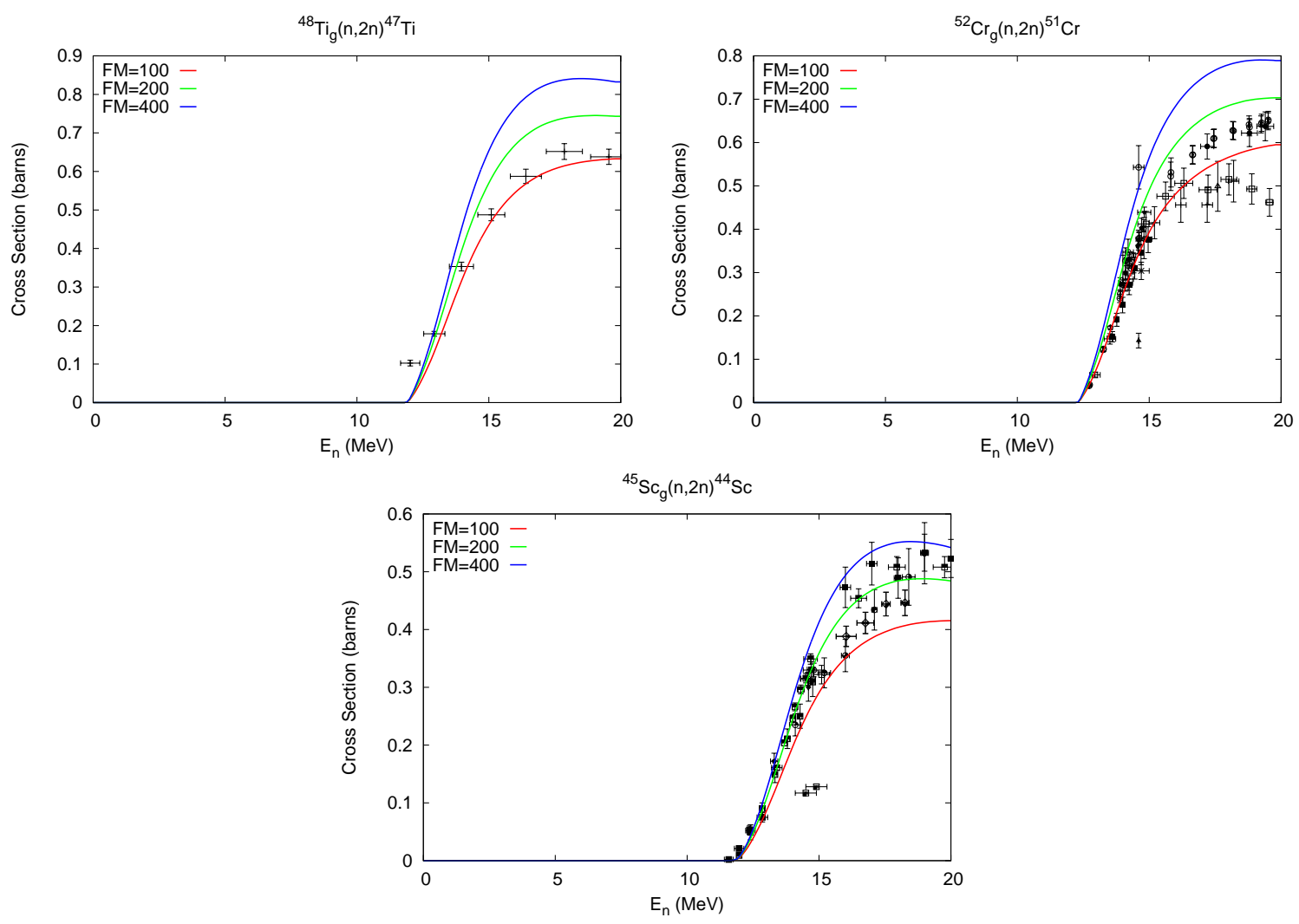

Fig. 15.- Sensitivity of $(\mathrm{n}, 2 \mathrm{n})$ cross sections to pre-equilibrium matrix element. The black data points are activation cross section data from (CSISRS 2003). The red, green, and blue lines correspond to our calculated activation cross section using $\langle F M\rangle$ of 100, 200, and 400, respectively.

sections on ${ }^{45} \mathrm{Sc},{ }^{48} \mathrm{Ti}$, and ${ }^{52} \mathrm{Cr}$. Only data for the activation cross section is shown. For comparison we show our local systematic level density (red-solid line, Appendix 9).

The second level density prescription (Rauscher et al. 1997) is plotted as the green solid line. This prescription is the current standard used in the calculation of cross sections for use in stellar nucleosynthesis calculations (Rauscher et al. 2002). This level density prescription is similar to ours, in that it also embodies an energy dependent $a(E)$ parameter (e.g. (Iljinov et al. 1992)) with shell corrections that damp out with increasing excitation energy (see our Eq. 14). It differs from ours in the parameterization of the asymptotic value of the $a$ parameter.

The last choice (blue-solid line) reflects a recent attempt to calculate level densities using microscopic nuclear structure models (Goriely 2002).

For the $(n, \gamma)$ capture reactions, we see that all three level density prescriptions result in a similar cross section below $5 \mathrm{MeV}$, although the overall scale varies. This is due primarily to the fact that the larger level densities will result in a larger calculated $s$-wave radiation width, and therefore require a smaller overall normalization to the gamma ray strength function. For this reason, we see that the shape of the cross section is the same at low energies (since the Hauser-Feshbach formula is using the discrete level data as opposed to the level density), but the size of the cross section varies. Each level density prescription does a reasonably good job in modeling the cross sections. Since our level density systematic is based on the local region of interest as opposed to a global prescription, we consider it to be preferable to the other two in this local region of the periodic chart.

For the $(\mathrm{n}, 2 \mathrm{n})$ reaction we see that the FermiGas level densities both predict roughly the same cross section over the entire range of excitation energy studied. Again, we consider our local systematic to be preferable to any of the other three.

The $(n, p)$ cross section simlilarly shows only minor sensitivites to the level density prescription, with variations only being on the order of $10-20 \%$ at the peak, usually much less. 

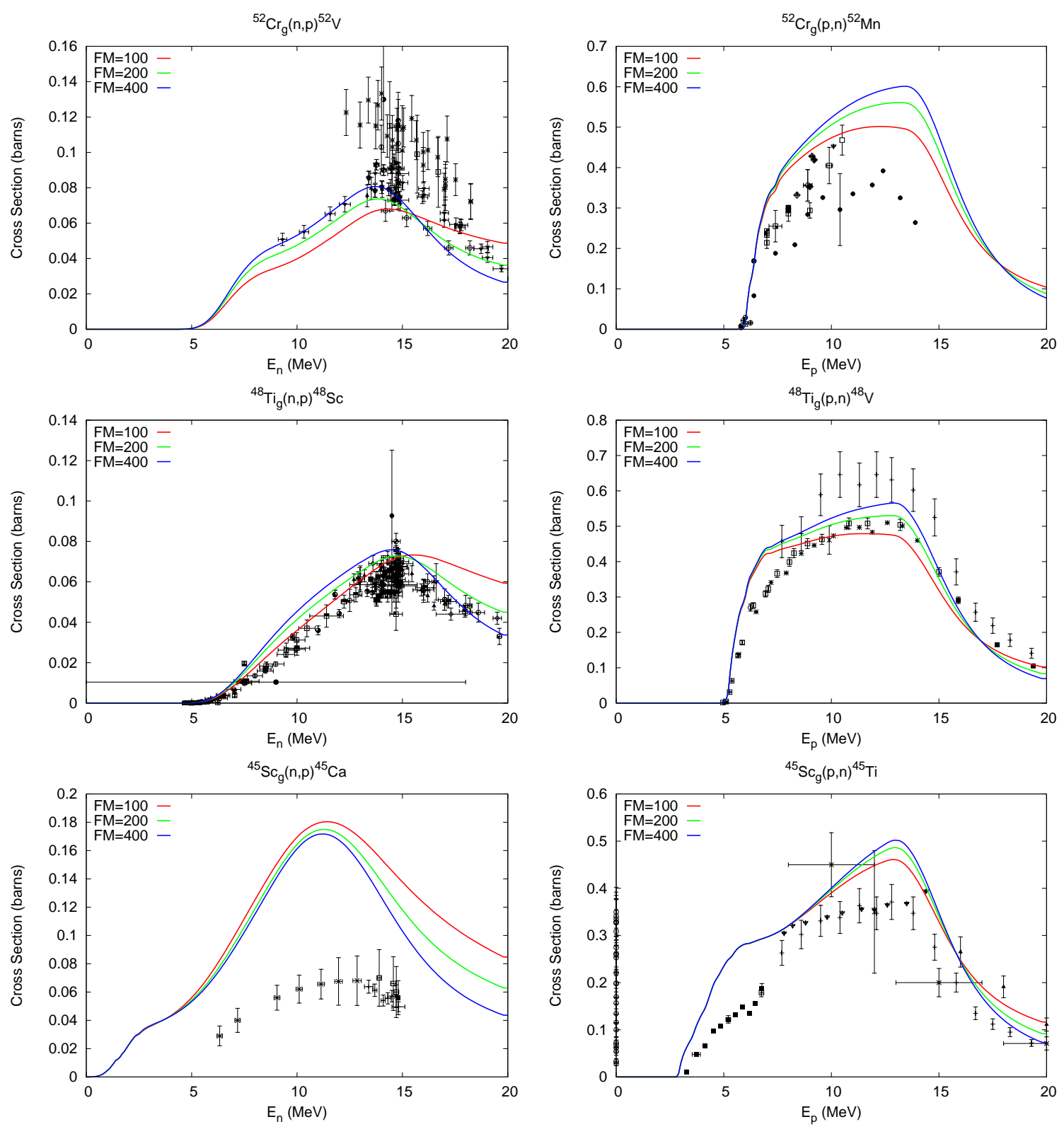

Fig. 16.- Sensitivity of $(\mathrm{n}, \mathrm{p})$ and $(\mathrm{p}, \mathrm{n})$ cross sections to pre-equilibrium matrix element. The black data points are activation cross section data from (CSISRS 2003). The red, green, and blue lines correspond to our calculated activation cross section using $\langle F M\rangle$ of 100,200 , and 400, respectively.

For the $(\mathrm{p}, \mathrm{n})$ cross section we see a more significant sensitivity to the level density prescription, especially around the peak. Our prescription replicates experimental data as well as the others in two of the three cases presented here, and better than the other two in the case of ${ }^{45} \mathrm{Sc}$. Even though our level density shows an improvement in the modeling of $(\mathrm{p}, \mathrm{n})$ cross sections in this region, we re-emphasize that the actual measured cross sections (West et al. 1993) will be used in the UGT analysis.

\subsubsection{Sensitivity to the Normalization of the $\gamma$ - ray Transmission Coefficient}

The $\gamma$-ray transmission coefficient is significantly smaller than the particle transmission coefficients that enter into the statistical model. Consequently, for neutron capture reactions, one finds that the cross section is roughly proportional to $T_{\gamma}$, multiplied by a relevent energy dependence. 

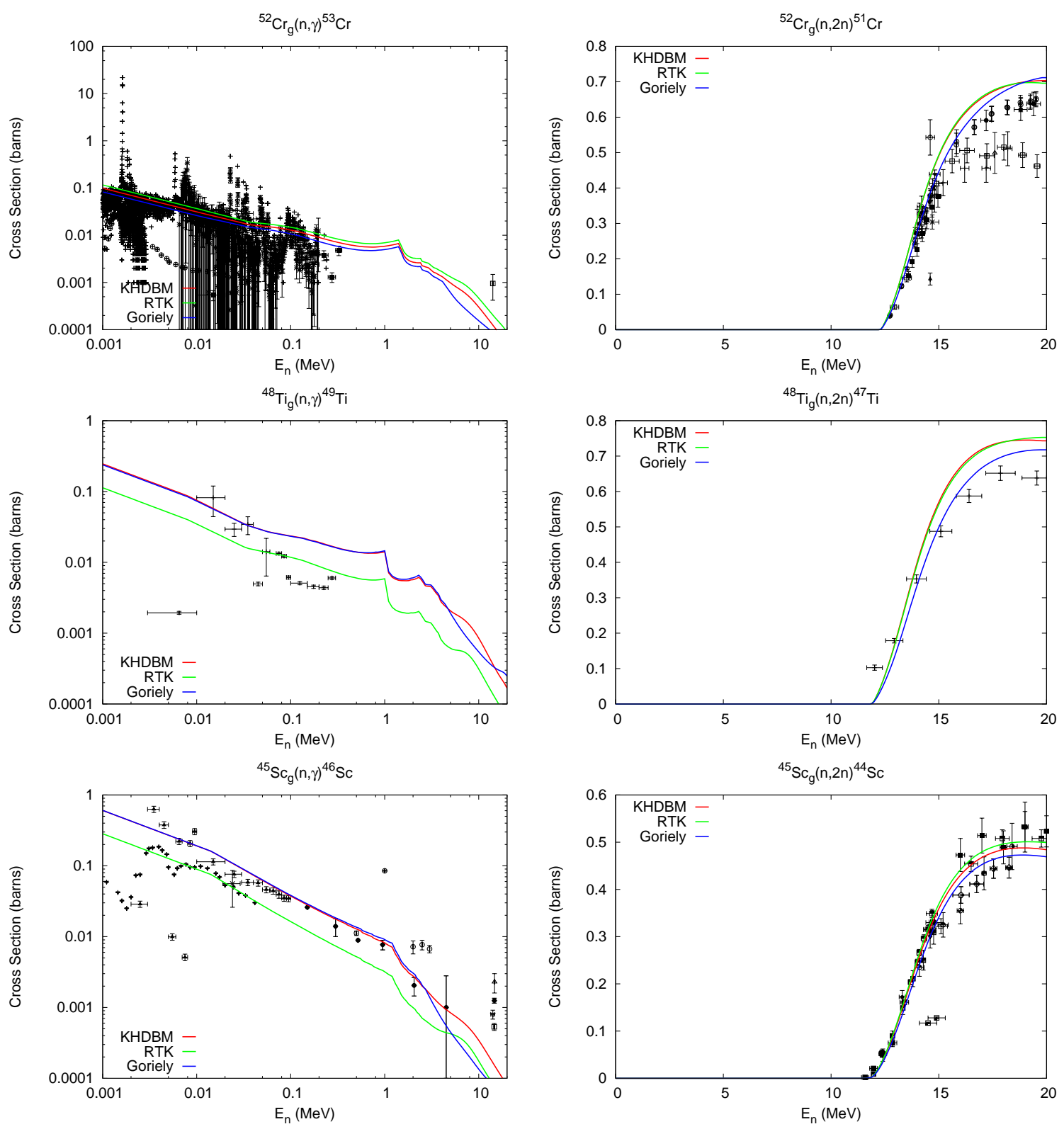

Fig. 17.- Sensitivity of $(n, \gamma)$ and $(n, 2 n)$ cross sections to the level density prescription. The black data corresponds to measured activation cross sections taken from (CSISRS 2003). The red solid line represents our calculation using our local level density systematic. The green and blue lines represent calculations using the level density prescriptions of (Rauscher et al. 1997) and (Goriely 2002), respectively.

Hence, one would expect that any adjustment to the normalization of the $\gamma$-ray transmission coefficient would have a corresponding effect on the capture cross sections. For instance, if the normalization constant is increased by a factor of two, the cross section should also increase by approximately a factor of two.

Figure 19 shows the sensitivity to a $\pm 30 \%$ change to the value of the average $s$-wave photon width used to normalize the gamma-ray transmission coefficients. This translates into a roughly $30 \%$ change in the cross section from $10 \mathrm{keV}$ to $1 \mathrm{MeV}$. The $(\mathrm{n}, 2 \mathrm{n})$ and $(\mathrm{p}, \mathrm{n})$ cross sections do not exhibit any sensitivity because $T_{\gamma}$, which is considerably smaller than the other transmission functions, only enters into Eq. [1] in the denominator. In general, the smaller of the two transmission coefficients in the HF numerator will be the 

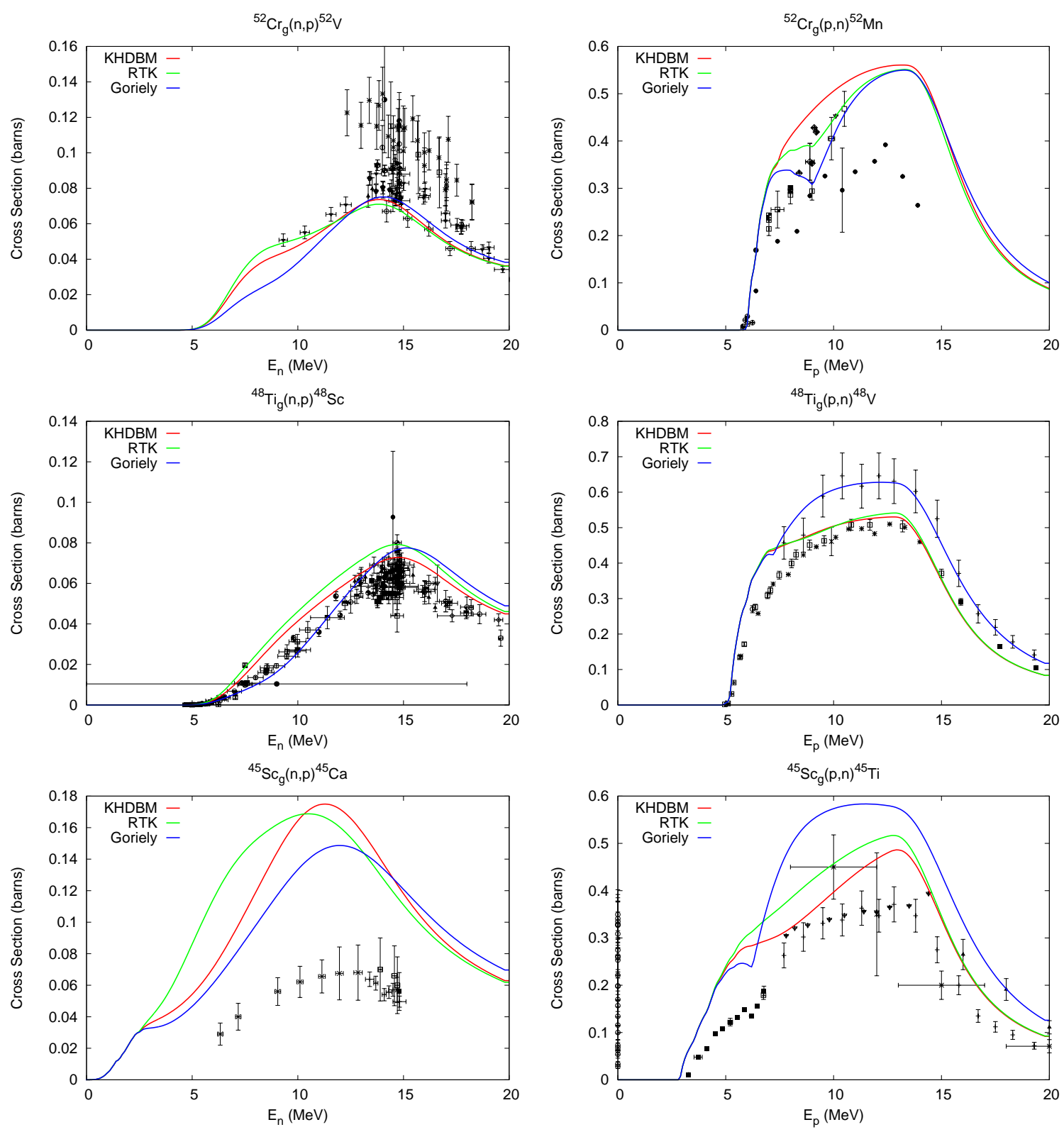

Fig. 18. - Sensitivity of $(\mathrm{n}, \mathrm{p})$ and $(\mathrm{p}, \mathrm{n})$ cross sections to the level density prescription. The black data corresponds to measured activation cross sections taken from (CSISRS 2003). The red solid line represents our calculation using our local level density systematic. The green and blue lines represent calculations using the global level density prescriptions of (Rauscher et al. 1997) and (Goriely 2002), respectively.

one that determines the cross section, especially if one is much smaller. This is always the case with photon vs. particle widths.

The mean error associated with the experimental values of the gamma ray strength function for isotopes ranging from calcium to cobalt is $31 \%$ with a standard deviation of $14 \%$. Hence, the uncertainties related to the gamma ray strength function in the capture cross sections will be gen- erally on the order of that shown in Figure 19.

\subsubsection{Sensitivity to the Inclusion of Width Fluc- tuation Corrections}

We adopt the Moldauer model of the WFC as embodied in the STAPRE code. For targets in the mass range of interest which have available experimental data, reaction thresholds are always greater than several $\mathrm{MeV}$, and width fluctuation 

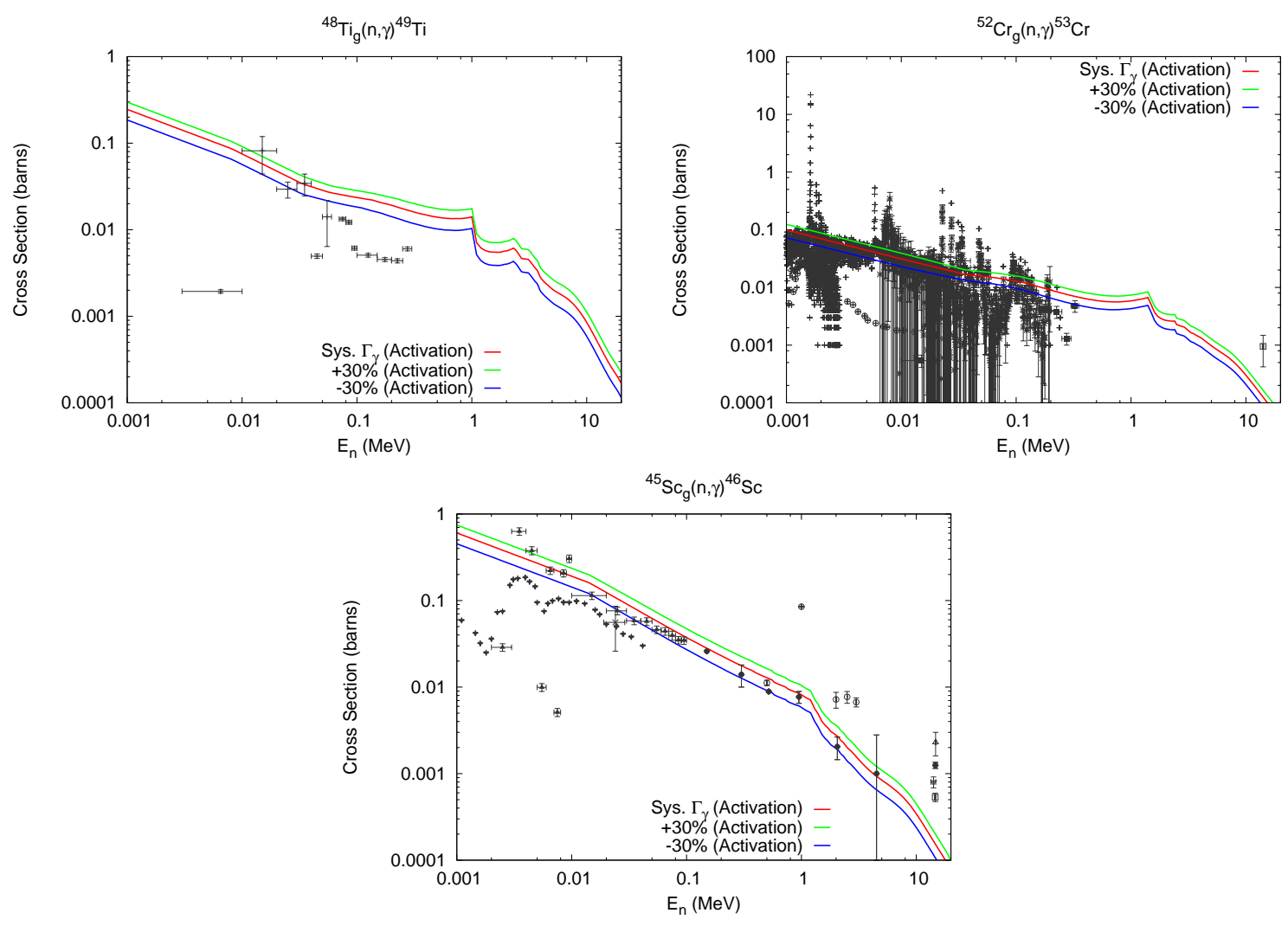

Fig. 19.- Sensitivity to a $\pm 30 \%$ adjustment of the experimental $s$-wave average photon width. The black data represents measured activation cross sections taken from (CSISRS 2003). The red line represents our calculation, and the green and blue lines represent our calculation with a $\pm 30 \%$ adjustment to the $s$-wave radiation width.

corrections will only be evident for capture reactions. Figure 20 shows the affect for the activation capture cross sections of ${ }^{45} \mathrm{Sc},{ }^{48} \mathrm{Ti}$, and ${ }^{52} \mathrm{Cr}$ both with (solid red line) and without (green dashed line) WFC. As expected a decrease in the capture cross section is noticeable, in both cases less than $20 \%$ below $100 \mathrm{keV}$. When the projectile energy increases, the capture cross section declines rapidly and the elastic enhancement vanishes. The $(n, p)$ and $(p, n)$ channels do not open until the incident neutron energy is well above $2 \mathrm{MeV}$, and so no other channels can compete with the elastic and capture cross sections before the WFC are negligible.

\subsection{Production and Destruction Cross Sections}

Figure 21 shows the modeled cross sections that directly affect the production and destruction of ${ }^{48} \mathrm{~V}$ and ${ }^{52} \mathrm{Mn}$. Identical plots for the other targets that span a slightly wider $(\mathrm{Z}, \mathrm{N})$ range of the original detector sets are presented in Appendix $\mathrm{C}$
(Figures 35 and 36).

The modeled $(\mathrm{p}, \mathrm{n})$ and $(\mathrm{d}, 2 \mathrm{n})$ cross sections are the production cross sections of primary importance, since ${ }^{48} \mathrm{Ti}$ and ${ }^{52} \mathrm{Cr}$ are the loaded isotopes. Secondary pathways exist that proceed through other isotopes, but these would require that the intermediate radioactive species be first populated though a series of $(\mathrm{p}, \mathrm{n})$ and/or $(\mathrm{n}, \mathrm{x})$ reactions from the loaded detector elements. Of course the particle fluences play a dominant role in determining the most important cross sections. More specific details will be disclosed in the classified analysis presented in a later paper.

Of the destruction cross sections, the largest are the $(n, p)$ and $(n, n p)+(n, p n)$. For many targets in this region, the $(\mathrm{n}, \mathrm{p})$ cross section does not have a threshold, and can be as much as an order of magnitude larger than the capture cross section. There are other nuclei for which the $(n, 2 n)$ and $(n, \gamma)$ reactions are the dominant destruction channels at energies above and below $\sim 5 \mathrm{MeV}$, respectively. Refer to Appendix $\mathrm{C}$ for the cross sections of great- 

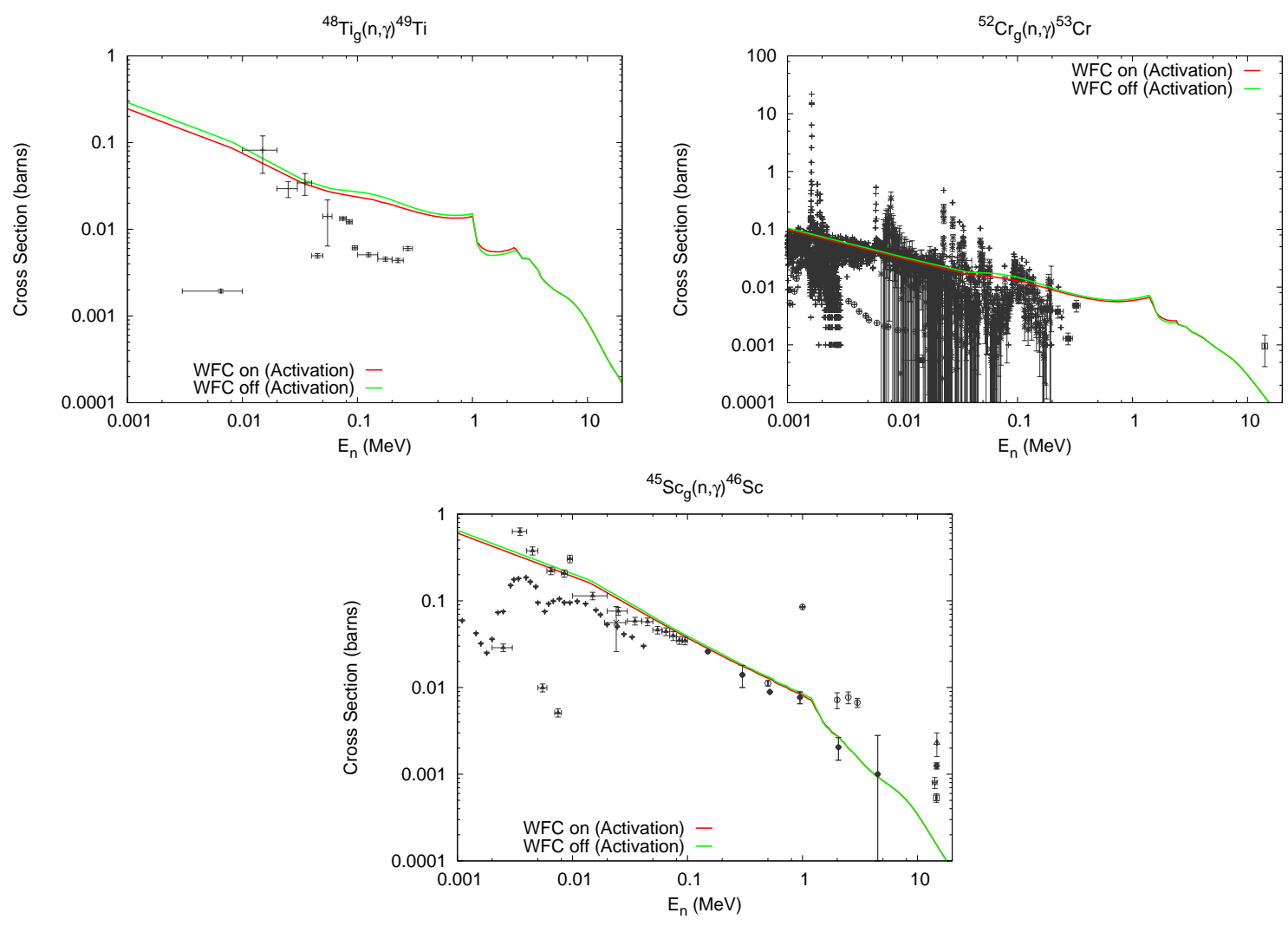

Fig. 20.- Sensitivity to inclusion or exclusion of width fluctuation corrections. The black data represents measured activation cross sections taken from (CSISRS 2003). The red line represents our calculation, and the green line represents our calculation excluding WFC.

est importance in the production and destruction of other isotopes.

\section{Conclusions}

We have developed a new charged particle cross section set for radiochemical diagnostics of ${ }^{48} \mathrm{~V}$ and ${ }^{52} \mathrm{Mn}$. The theory and implementation of the Hauser-Feshbach model were described (Section 2 ), along with the details of the local systematics used to create a set of input parameters that reflect the latest available experimental data in the region of interest (Section 3). Sensitivity of the modeled cross sections to the input models and their parameters has been explored (Section 4). The choice of our developed local systematics appears to do reasonably well in replicating measured cross sections over this restricted region of interest (Figures $6,7,8,10,11,12,14$, Appendix B). Previous efforts have performed a similar analysis for three other RADCHEM charged particle detector sets, ${ }^{79} \mathrm{Br}$ producing ${ }^{79} \mathrm{Kr}$ (Hoffman et al. 2004), ${ }^{127} \mathrm{I}$ producing ${ }^{127} \mathrm{Xe}$ (Hoffman et al. 2004), and stable Europium producing ${ }^{147-150,152,154} \mathrm{Eu}$ and
151,153 Gd (Hoffman et al. 2004).

Overall we consider the modeling effort to be quite successful, as our calculated cross sections do agree favorably with experimentally measured ones in this region of interest. In particular, we have demonstrated an ability to calculate $(\mathrm{n}, 2 \mathrm{n})$ cross sections to about 10-15\% accuracy (Table $4)$ and $(n, \gamma)$ reactions to within roughly a factor of two. Our results in previous modeling efforts proved to be somewhat more accurate (for capture cross sections). However, in this region of interest, we have to deal with the proximity of two closed neutron shells and one closed proton shell. Additionally, the measured resonance parameters, particularly the average $s$-wave radiation widths to which the photon transmission functions are normalized, have considerably larger errors.

Our modeled results are generally in good agreement with measured neutron induced cross sections with charged particles in the exit channel. Our (n,p) reactions are generally within $30 \%$ of measured values. We also find satisfactory comparisons between our calculations and measured 

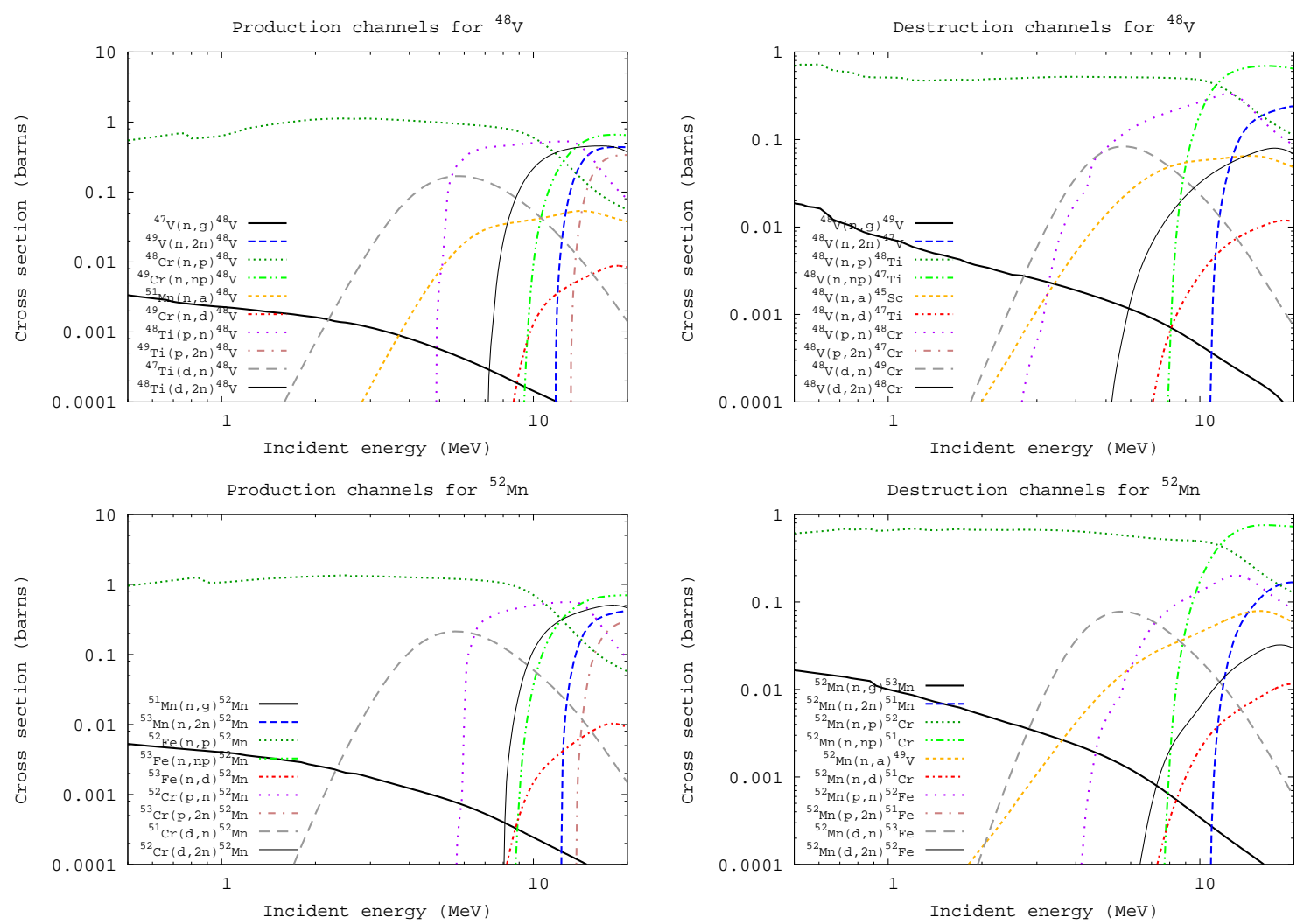

Fig. 21. - Calculated cross sections directly affecting production and destruction of ${ }^{48} \mathrm{~V}$ and ${ }^{52} \mathrm{Mn}$.

charged-particle induced reactions. In particular, the $(\mathrm{p}, \mathrm{n})$ reactions are generally within $15-20 \%$. We note that the charged particle reactions of greatest import to the UGT program have been measured, and will be used in preference to our modeled results in that analysis.

We have made a special effort to explicitly calculate all possible neutron induced destruction cross sections and note that $(n, p)$ cross sections are much larger than $(n, \gamma)$ for many species of interest in this mass range (Appendix $\mathrm{C}$ ). For the Ti0887 and Cr0386 sets, neither ${ }^{48} \mathrm{~V}(\mathrm{n}, \mathrm{p})^{48} \mathrm{Ti}$ nor ${ }^{52} \mathrm{Mn}(\mathrm{n}, \mathrm{p})^{52} \mathrm{Cr}$, here identified as the dominant destruction reactions on the radioactivities of specific interest to radchem, were explicitly calculated. For both radio-isotopes, the (n,p) destruction cross section is orders of magnitude larger at nearly all incident particle energies than that through neutron capture (Figure 21). Our analysis indicates that the largest uncertainty in our calculated $(n, p)$ cross sections lies in the prescription of the proton optical potential (§3.2.3, Figure 2 ). It was shown that this may be in error by as much as a factor of two (on the high side). Even with a $50 \%$ reduction, the $(\mathrm{n}, \mathrm{p})$ cross section is still larger than $(n, \gamma)$ by an order of magnitude, which could have important ramifications on radiochemical analysis.

Determining either one of these cross sections would provide for detector set(s) in which all the important production and destruction cross sections are measured. In 2005, a LLNL-LANL-RPI collaboration led by Prof. Yaron Danon proposed a direct measurement of the ${ }^{48} \mathrm{~V}(\mathrm{n}, \mathrm{p})^{48} \mathrm{Ti}$ cross section utilizing LANL's Isotope Production Facility for target fabrication and the Lead Slowing Down Spectrometer at LANCE. The experiment will take some time to perform, but we consider this to be of great importance to our radiochemistry effort.

\section{Acknowledgments}

This work was performed under the auspices of the U.S. Department of Energy by the University of California Lawrence Livermore National Laboratory under contract W-7405-ENG-48. 


\section{REFERENCES}

Avrigeanu, M. \& Avrigeanu, V. IPNE-Bucharest Report No. NP-86-1995 (September 1995) http://www.nea.fr/abs/html/iaea0971.html

Bao, Z. Y., Beer, H., Kappeler, F.,, Voss, F., \& Wisshak, K. 2000, Atomic Data \& Nuclear Data Tables, 76, 70

Bauer, R. 2001, private communication.

Beer, H., Voss, F., \& Winters, R. R. 1992, ApJS, 80, 403

Bohr, A., \& Mottelson, B. Nuclear Structure Vol. 1, Single-Particle Motion, World Scientific, 1998

Burgus, W.H. et al. , 1954, Phys. Rev. 95, 750

Experimental Nuclear Reaction Data File, Brookhaven National Laboratory, US Dept. of Energy, http://www.nndc.bnl.gov/nndc/exfor/

Chadwick, M. 1998, the GNASH statistical model code, http://www.nea.fr/abs/html/psr-0125.html

Cline, C. K. 1972, Nucl. Phys. A195, 353

Dietrich, F.S., 2001, private communication.

Dugersuren, D., Mitchell, G. E., Garrett, P., Agvaanluvsan, U., Becker, J., bernstein, L., Cooper, J., Hoffman, R., Macri, R.. Younes, W., Devlin, M., Fotiades, N., \& Nelson, R. 2005, " ${ }^{48} \mathrm{Ti}(n, x n p \alpha \gamma)$ reaction cross section using spallation neutrons for $\mathrm{E}_{n}=1-20 \mathrm{MeV}$ ". UCRL-TR-209474, LLNL.

ENSDF: Evaluated Nuclear Structure Data File, Brookhaven National Laboratory, US Dept. of Energy, http://www.nndc.bnl.gov/nndc/ensdf/

Gardner, M.A. \& Howerton, R.J., 1978, "ACTL: Evaluated Neutron Activation Cross-Section Library", UCRL-50400

Gilbert, A., \& Cameron, A.G.W. 1965, Can. J. Phys., 43, 1446

Demetriou, P., \& Goriely, S. 2001, Nucl. Phys. A695, 95

Hilaire, S., Lagrange, Ch., \& Koning, A. J. 2003, Ann. of Phys. 306, 209

Hofmann, H.M., Richert, J, Tepel, J. W. \& Weidenmuller, H.A. 1975, Ann. of Phys. 90, 403

Hoffman, R. D., Rauscher, T., Woosley, S. E. \& Thielemann, F.-K. 1999, ApJ, 521, 735

Hoffman, R.D., Dietrich, F.S., Bauer, R., Kelley, K., and Mustafa, M. 2004 UCRL-TR-205563 (Br-Kr)

Hoffman, R.D., Dietrich, F.S., Bauer, R., Kelley, K., and Mustafa, M. 2004 UCRL-TR-206721 (I-Xe)

Hoffman, R.D., Dietrich, F.S., Bauer, R., Kelley, K., and Mustafa, M. 2004 UCRL-TR-211558 (Sm-Eu-Gd)

Iljinov, A.S., Mebel, M.V., Bianchi, N., De Sanctis, E., Guaraldo, C., Lucherini, V., Muccifora, V., Polli, E., Reolon, A.R., \& Rossi, P. 1992, Nucl. Phys. A543, 517

Keisch, B. 1963, Phys. Rev. 129, 769

Koning, A.J., \& Delaroche, J.P. 2003, Nucl. Phys. A713, 231

McFadden, L., and Satchler, G.R., 1966, Nuc. Phys. 84, 177

Mclane, V., Dunford, C., \& Rose, P. Neutron Cross Sections, Vol. 2, Neutron Cross Section Curves, Academic Press, 1988

Milazzo-Colli, L., and Braga-Marcazzan, G.M., 1973, Nuc. Phys. A210, 297 
Moldauer, P. A. 1976 Phys. Rev. C14 764

Möller, P., Nix, J.R., Myers, W.D., \& Swiatecki, W.J. 1995, Atomic Data \& Nuclear Data Tables, 59, 185

Mughabghab, S. F. and Garber, D. I., Neutron Cross Sections, Brookhaven National Laboratory, 1973, BNL-325/ED3

Mughabghab, S. F., Divadeenam, M., and Holden, N.E., Neutron Cross Sections, 1982, Academic Press, New York

Mustafa, M., Nuclear Data for Science and Technology Proc. 59, p. 274, 1997, Trieste

Nethaway, D.R., Memo, "The Cross-Section Sets Used With the Watusi Program." LLNL A-Division memo, 5 Nov., 1998

Perey, C.M. and Perey, F.G., 1963, Phys. Rev. 132, 755

Rauscher, T., Thielemann, F.-K., \& Kratz, K.-L. 1997, Phys. Rev. C., 56, 1613

Rauscher, T., Thielemann, F.-K. 2000, Atomic Data \& Nuclear Data Tables, 79, 47

Rauscher, T., Heger, A. Hoffman, R. D. \& Woosley, S. E. 2002, ApJ, 576, 323

Reffo, G. 1978, ICTP Lecture Series, 17 Jan. - 10 Mar., Trieste

Raynal, J. "ECIS96", Proceedings of the Specialists' Meeting on the Nucleon Nucleus Optical Model up to $200 \mathrm{MeV}, 13-15$ November 1996, Bruyeres-le-Chatel, Franc (http://www.nea.fr/html/science/om200/raynal.pdf)

Handbook for calculations of nuclear reaction data, Reference input parameter library. 1998, IAEA-TECDOC1034

Woosley, S. E., Fowler, W. A., Holmes, J. A. \& Zimmerman, B. A. 1978, Atomic Data \& Nuclear Data Tables, 22, 371

Udagawa, T., \& Tamura, T., Phys. Rev. C 24, 1348 (1981); 33, 494(1986)

Uhl, M., \& Strohmaier, B. IRK-Vienna Report IRK-76/01 1976 (Upd. 1978)

Vonach, H. 1982, UCID-19549, LLNL

Walter, G. et. al. 1986, Nucl Sci. Eng., 93, 357

West, H., Lanier, R., Mustafa, M., Nuckolls, R., Nagle, R., O'Brien, H., Frehaut, J., Adam, A., and Philis, C., 1993, UCRL-ID-115738

Williams, F. C. Jr. 1970, Phys. Lett. 31B, 184

This 2-column preprint was prepared with the AAS LATEX macros v5.0. 


\section{A. Basic Nuclear Structure Data}

A.1. New Scandium, Titanium-Vanadium, Chromium-Manganese, and Iron Detector Sets

Table 5:: Neutron induced reactions calculated for new scandium, titanium, chromium, and iron sets

\begin{tabular}{|c|c|c|c|c|c|c|c|c|c|}
\hline${ }^{{ }^{A} Z}$ & lifetime & $\overline{(n, 2 n)}$ & $\overline{(n, 3 n)}$ & $\overline{\left(\mathrm{n}, \mathrm{n}^{\prime}\right)}$ & $\overline{(n, \gamma)}$ & $\overline{(n, p)}$ & $\overline{(n, n+p)}$ & $\overline{(\mathrm{n}, \alpha)}$ & $\overline{(\mathrm{n}, \mathrm{d})}$ \\
\hline${ }^{41} \mathrm{Ca}$ & $1.03 \times 10^{5} \mathrm{y}$ & - & & & - & - & - & - & - \\
\hline${ }^{42} \mathrm{Ca}$ & $0.647 \%$ & - & & & - & - & - & - & - \\
\hline${ }^{43} \mathrm{Ca}$ & $0.135 \%$ & - & - & & - & - & - & - & - \\
\hline${ }^{44} \mathrm{Ca}$ & $2.09 \%$ & - & - & & - & - & - & - & - \\
\hline${ }^{45} \mathrm{Ca}$ & $162.61 \mathrm{~d}$ & $\bullet$ & $\bullet$ & & $\bullet$ & $\bullet$ & $\bullet$ & $\bullet$ & $\bullet$ \\
\hline${ }^{46} \mathrm{Ca}$ & $0.004 \%$ & $\bullet$ & $\bullet$ & & $\bullet$ & $\bullet$ & $\bullet$ & $\bullet$ & $\bullet$ \\
\hline${ }^{47} \mathrm{Ca}$ & $4.536 \mathrm{~d}$ & $\bullet$ & $\bullet$ & & $\bullet$ & $\bullet$ & $\bullet$ & $\bullet$ & $\bullet$ \\
\hline${ }^{48} \mathrm{Ca}$ & $0.187 \%$ & $\bullet$ & $\bullet$ & & $\bullet$ & $\bullet$ & $\bullet$ & $\bullet$ & $\bullet$ \\
\hline${ }^{41} \mathrm{Sc}$ & $596.3 \mathrm{~ms}$ & - & & & - & - & - & - & - \\
\hline${ }^{42} \mathrm{Sc}$ & $680.67 \mathrm{~ms}$ & - & & - & - & - & - & - & - \\
\hline${ }^{42} \mathrm{Sc}_{m}$ & $61.7 \mathrm{~s}$ & $\bullet$ & & $\bullet$ & $\bullet$ & $\bullet$ & $\bullet$ & $\bullet$ & $\bullet$ \\
\hline${ }^{43} \mathrm{Sc}$ & $3.891 \mathrm{~h}$ & $\bullet$ & & & $\bullet$ & $\bullet$ & $\bullet$ & $\bullet$ & $\bullet$ \\
\hline${ }^{44} \mathrm{Sc}$ & $3.97 \mathrm{~h}$ & $\bullet$ & & $\bullet$ & $\bullet$ & $\bullet$ & $\bullet$ & $\bullet$ & $\bullet$ \\
\hline${ }^{44} \mathrm{Sc}_{m}$ & $58.61 \mathrm{~h}$ & $\bullet$ & & $\bullet$ & $\bullet$ & $\bullet$ & $\bullet$ & $\bullet$ & $\bullet$ \\
\hline${ }^{45} \mathrm{Sc}$ & $100 \%$ & $\bullet$ & & $\bullet$ & $\bullet$ & $\bullet$ & $\bullet$ & $\bullet$ & $\bullet$ \\
\hline${ }^{45} \mathrm{Sc}_{m}$ & $318 \mathrm{~ms}$ & $\bullet$ & & $\bullet$ & $\bullet$ & $\bullet$ & $\bullet$ & $\bullet$ & $\bullet$ \\
\hline${ }^{46} \mathrm{Sc}$ & $83.79 \mathrm{~d}$ & $\bullet$ & & $\bullet$ & $\bullet$ & $\bullet$ & $\bullet$ & $\bullet$ & $\bullet$ \\
\hline${ }^{46} \mathrm{Sc}_{m}$ & $18.75 \mathrm{~s}$ & $\bullet$ & & $\bullet$ & $\bullet$ & $\bullet$ & $\bullet$ & $\bullet$ & $\bullet$ \\
\hline${ }^{47} \mathrm{Sc}$ & $3.3492 \mathrm{~d}$ & $\bullet$ & $\bullet$ & & $\bullet$ & $\bullet$ & $\bullet$ & $\bullet$ & $\bullet$ \\
\hline${ }^{48} \mathrm{Sc}$ & $43.67 \mathrm{~h}$ & $\bullet$ & $\bullet$ & & $\bullet$ & $\bullet$ & $\bullet$ & $\bullet$ & $\bullet$ \\
\hline${ }^{49} \mathrm{Sc}$ & $57.2 \mathrm{~m}$ & $\bullet$ & $\bullet$ & & $\bullet$ & $\bullet$ & $\bullet$ & $\bullet$ & $\bullet$ \\
\hline${ }^{50} \mathrm{Sc}$ & $102.5 \mathrm{~s}$ & $\bullet$ & $\bullet$ & $\bullet$ & $\bullet$ & $\bullet$ & $\bullet$ & $\bullet$ & $\bullet$ \\
\hline${ }^{50} \mathrm{Sc}_{m}$ & $0.35 \mathrm{~s}$ & $\bullet$ & $\bullet$ & $\bullet$ & $\bullet$ & $\bullet$ & $\bullet$ & $\bullet$ & $\bullet$ \\
\hline${ }^{44} \mathrm{Ti}$ & $60.0 \mathrm{y}$ & $\bullet$ & & & $\bullet$ & $\bullet$ & $\bullet$ & $\bullet$ & $\bullet$ \\
\hline${ }^{45} \mathrm{Ti}$ & $184.8 \mathrm{~m}$ & $\bullet$ & & & $\bullet$ & $\bullet$ & $\bullet$ & $\bullet$ & $\bullet$ \\
\hline${ }^{46} \mathrm{Ti}$ & $8.25 \%$ & $\bullet$ & & & $\bullet$ & $\bullet$ & $\bullet$ & $\bullet$ & $\bullet$ \\
\hline${ }^{47} \mathrm{Ti}$ & $7.44 \%$ & $\bullet$ & & & $\bullet$ & $\bullet$ & $\bullet$ & $\bullet$ & $\bullet$ \\
\hline${ }^{48} \mathrm{Ti}$ & $73.72 \%$ & $\bullet$ & & & $\bullet$ & $\bullet$ & $\bullet$ & $\bullet$ & $\bullet$ \\
\hline${ }^{49} \mathrm{Ti}$ & $5.41 \%$ & $\bullet$ & $\bullet$ & & $\bullet$ & $\bullet$ & $\bullet$ & $\bullet$ & $\bullet$ \\
\hline${ }^{50} \mathrm{Ti}$ & $5.18 \%$ & $\bullet$ & $\bullet$ & & $\bullet$ & $\bullet$ & $\bullet$ & $\bullet$ & $\bullet$ \\
\hline${ }^{51} \mathrm{Ti}$ & $5.76 \mathrm{~m}$ & $\bullet$ & $\bullet$ & & $\bullet$ & $\bullet$ & $\bullet$ & $\bullet$ & $\bullet$ \\
\hline${ }^{52} \mathrm{Ti}$ & $1.7 \mathrm{~m}$ & $\bullet$ & $\bullet$ & & $\bullet$ & $\bullet$ & $\bullet$ & $\bullet$ & $\bullet$ \\
\hline${ }^{46} \mathrm{~V}$ & $422.50 \mathrm{~ms}$ & $\bullet$ & & $\bullet$ & $\bullet$ & $\bullet$ & $\bullet$ & $\bullet$ & $\bullet$ \\
\hline${ }^{46} \mathrm{~V}_{m}$ & $1 \mathrm{~ms}$ & - & & - & - & - & - & - & - \\
\hline${ }^{47} \mathrm{~V}$ & $32.6 \mathrm{~m}$ & $\bullet$ & & & $\bullet$ & $\bullet$ & $\bullet$ & $\bullet$ & $\bullet$ \\
\hline${ }^{48} \mathrm{~V}$ & $15.9735 \mathrm{~d}$ & $\bullet$ & & & $\bullet$ & $\bullet$ & $\bullet$ & $\bullet$ & $\bullet$ \\
\hline${ }^{49} \mathrm{~V}$ & $330 \mathrm{~d}$ & $\bullet$ & & & $\bullet$ & $\bullet$ & $\bullet$ & $\bullet$ & $\bullet$ \\
\hline${ }^{50} \mathrm{~V}$ & $0.250 \%$ & $\bullet$ & & & $\bullet$ & $\bullet$ & $\bullet$ & $\bullet$ & $\bullet$ \\
\hline${ }^{51} \mathrm{~V}$ & $99.750 \%$ & $\bullet$ & & & $\bullet$ & $\bullet$ & $\bullet$ & $\bullet$ & $\bullet$ \\
\hline${ }^{52} \mathrm{~V}$ & $3.743 \mathrm{~m}$ & $\bullet$ & $\bullet$ & & $\bullet$ & $\bullet$ & $\bullet$ & $\bullet$ & $\bullet$ \\
\hline${ }^{53} \mathrm{~V}$ & $1.60 \mathrm{~m}$ & $\bullet$ & $\bullet$ & & $\bullet$ & $\bullet$ & $\bullet$ & $\bullet$ & $\bullet$ \\
\hline${ }^{48} \mathrm{Cr}$ & $21.56 \mathrm{~h}$ & $\bullet$ & & & $\bullet$ & $\bullet$ & $\bullet$ & $\bullet$ & $\bullet$ \\
\hline${ }^{49} \mathrm{Cr}$ & $42.3 \mathrm{~m}$ & - & & & - & - & - & - & - \\
\hline${ }^{50} \mathrm{Cr}$ & $4.345 \%$ & $\bullet$ & & & $\bullet$ & $\bullet$ & $\bullet$ & $\bullet$ & $\bullet$ \\
\hline${ }^{51} \mathrm{Cr}$ & $27.7025 \mathrm{~d}$ & $\bullet$ & & & $\bullet$ & $\bullet$ & $\bullet$ & $\bullet$ & $\bullet$ \\
\hline${ }^{52} \mathrm{Cr}$ & $83.789 \%$ & - & & & - & - & - & - & - \\
\hline
\end{tabular}


Table 5: (continued)

\begin{tabular}{|c|c|c|c|c|c|c|c|c|c|}
\hline${ }^{A} Z$ & lifetime & $(\mathrm{n}, 2 \mathrm{n})$ & $(n, 3 n)$ & $\left(n, n^{\prime}\right)$ & $(\mathrm{n}, \gamma)$ & $(\mathrm{n}, \mathrm{p})$ & $(\mathrm{n}, \mathrm{n}+\mathrm{p})$ & $(\mathrm{n}, \alpha)$ & $(\mathrm{n}, \mathrm{d})$ \\
\hline${ }^{53} \mathrm{Cr}$ & $9.501 \%$ & $\bullet$ & & & $\bullet$ & $\bullet$ & $\bullet$ & $\bullet$ & $\bullet$ \\
\hline${ }^{54} \mathrm{Cr}$ & $2.365 \%$ & $\bullet$ & $\bullet$ & & $\bullet$ & $\bullet$ & $\bullet$ & $\bullet$ & $\bullet$ \\
\hline${ }^{55} \mathrm{Cr}$ & $3.497 \mathrm{~m}$ & $\bullet$ & $\bullet$ & & $\bullet$ & $\bullet$ & $\bullet$ & $\bullet$ & $\bullet$ \\
\hline${ }^{56} \mathrm{Cr}$ & $5.94 \mathrm{~m}$ & $\bullet$ & $\bullet$ & & $\bullet$ & $\bullet$ & $\bullet$ & $\bullet$ & $\bullet$ \\
\hline${ }^{50} \mathrm{Mn}$ & $283.29 \mathrm{~ms}$ & $\bullet$ & & $\bullet$ & $\bullet$ & $\bullet$ & $\bullet$ & $\bullet$ & $\bullet$ \\
\hline${ }^{50} \mathrm{Mn}_{m}$ & $1.75 \mathrm{~m}$ & $\bullet$ & & $\bullet$ & $\bullet$ & $\bullet$ & $\bullet$ & $\bullet$ & $\bullet$ \\
\hline${ }^{51} \mathrm{Mn}$ & $46.2 \mathrm{~m}$ & $\bullet$ & & & $\bullet$ & $\bullet$ & $\bullet$ & $\bullet$ & $\bullet$ \\
\hline${ }^{52} \mathrm{Mn}$ & $5.591 \mathrm{~d}$ & $\bullet$ & & $\bullet$ & $\bullet$ & $\bullet$ & $\bullet$ & $\bullet$ & $\bullet$ \\
\hline${ }^{52} \mathrm{Mn}_{m}$ & $21.1 \mathrm{~m}$ & $\bullet$ & & $\bullet$ & $\bullet$ & $\bullet$ & $\bullet$ & $\bullet$ & $\bullet$ \\
\hline${ }^{53} \mathrm{Mn}$ & $3.74 \times 10^{6} \mathrm{y}$ & $\bullet$ & & & $\bullet$ & $\bullet$ & $\bullet$ & $\bullet$ & $\bullet$ \\
\hline${ }^{54} \mathrm{Mn}$ & $312.11 \mathrm{~d}$ & $\bullet$ & & & $\bullet$ & $\bullet$ & $\bullet$ & $\bullet$ & $\bullet$ \\
\hline${ }^{55} \mathrm{Mn}$ & $100 \%$ & $\bullet$ & $\bullet$ & & $\bullet$ & $\bullet$ & $\bullet$ & $\bullet$ & $\bullet$ \\
\hline${ }^{56} \mathrm{Mn}$ & $2.5789 \mathrm{~h}$ & $\bullet$ & $\bullet$ & & $\bullet$ & $\bullet$ & $\bullet$ & $\bullet$ & $\bullet$ \\
\hline${ }^{57} \mathrm{Mn}$ & $85.4 \mathrm{~s}$ & $\bullet$ & $\bullet$ & & $\bullet$ & $\bullet$ & $\bullet$ & $\bullet$ & $\bullet$ \\
\hline${ }^{52} \mathrm{Fe}$ & $8.275 \mathrm{~h}$ & $\bullet$ & & $\bullet$ & $\bullet$ & $\bullet$ & $\bullet$ & $\bullet$ & $\bullet$ \\
\hline${ }^{53} \mathrm{Fe}$ & $8.51 \mathrm{~m}$ & $\bullet$ & & $\bullet$ & $\bullet$ & $\bullet$ & $\bullet$ & $\bullet$ & $\bullet$ \\
\hline${ }^{54} \mathrm{Fe}$ & $5.845 \%$ & $\bullet$ & & & $\bullet$ & $\bullet$ & $\bullet$ & $\bullet$ & $\bullet$ \\
\hline${ }^{55} \mathrm{Fe}$ & $2.73 \mathrm{y}$ & $\bullet$ & & & $\bullet$ & $\bullet$ & $\bullet$ & $\bullet$ & $\bullet$ \\
\hline${ }^{56} \mathrm{Fe}$ & $91.754 \%$ & $\bullet$ & & & $\bullet$ & $\bullet$ & $\bullet$ & $\bullet$ & $\bullet$ \\
\hline${ }^{57} \mathrm{Fe}$ & $2.119 \%$ & $\bullet$ & $\bullet$ & & $\bullet$ & $\bullet$ & $\bullet$ & $\bullet$ & • \\
\hline${ }^{58} \mathrm{Fe}$ & $0.282 \%$ & $\bullet$ & $\bullet$ & & $\bullet$ & $\bullet$ & $\bullet$ & $\bullet$ & $\bullet$ \\
\hline
\end{tabular}

Table 6:: Charged-particle induced reactions calculated for new scandium, titanium, chromium, and iron sets

\begin{tabular}{|c|c|c|c|c|c|}
\hline${ }^{A} Z$ & lifetime & $(\mathrm{p}, \mathrm{n})$ & $(\mathrm{p}, 2 \mathrm{n})$ & $(\mathrm{d}, \mathrm{n})$ & $\overline{(d, 2 n)}$ \\
\hline${ }^{41} \mathrm{Ca}$ & $1.03 \times 10^{5} \mathrm{y}$ & $\bullet$ & & $\bullet$ & $\bullet$ \\
\hline${ }^{42} \mathrm{Ca}$ & $0.647 \%$ & $\bullet$ & $\bullet$ & $\bullet$ & $\bullet$ \\
\hline${ }^{43} \mathrm{Ca}$ & $0.135 \%$ & $\bullet$ & $\bullet$ & $\bullet$ & $\bullet$ \\
\hline${ }^{44} \mathrm{Ca}$ & $2.09 \%$ & $\bullet$ & $\bullet$ & $\bullet$ & $\bullet$ \\
\hline${ }^{45} \mathrm{Ca}$ & $162.61 \mathrm{~d}$ & $\bullet$ & $\bullet$ & $\bullet$ & $\bullet$ \\
\hline${ }^{46} \mathrm{Ca}$ & $0.004 \%$ & $\bullet$ & $\bullet$ & $\bullet$ & $\bullet$ \\
\hline${ }^{47} \mathrm{Ca}$ & $4.536 \mathrm{~d}$ & $\bullet$ & $\bullet$ & $\bullet$ & $\bullet$ \\
\hline${ }^{48} \mathrm{Ca}$ & $0.187 \%$ & $\bullet$ & $\bullet$ & $\bullet$ & $\bullet$ \\
\hline${ }^{41} \mathrm{Sc}$ & $596.3 \mathrm{~ms}$ & $\bullet$ & & $\bullet$ & $\bullet$ \\
\hline${ }^{42} \mathrm{Sc}$ & $680.67 \mathrm{~ms}$ & $\bullet$ & & $\bullet$ & $\bullet$ \\
\hline${ }^{42} \mathrm{Sc}_{m}$ & $61.7 \mathrm{~s}$ & $\bullet$ & & $\bullet$ & $\bullet$ \\
\hline${ }^{43} \mathrm{Sc}$ & $3.891 \mathrm{~h}$ & $\bullet$ & & $\bullet$ & $\bullet$ \\
\hline${ }^{44} \mathrm{Sc}$ & $3.97 \mathrm{~h}$ & $\bullet$ & $\bullet$ & $\bullet$ & $\bullet$ \\
\hline${ }^{44} \mathrm{Sc}_{m}$ & $58.61 \mathrm{~h}$ & $\bullet$ & $\bullet$ & $\bullet$ & $\bullet$ \\
\hline${ }^{45} \mathrm{Sc}$ & $100 \%$ & $\bullet$ & $\bullet$ & $\bullet$ & $\bullet$ \\
\hline${ }^{45} \mathrm{Sc}_{m}$ & $318 \mathrm{~ms}$ & $\bullet$ & $\bullet$ & $\bullet$ & $\bullet$ \\
\hline${ }^{46} \mathrm{Sc}$ & $83.79 \mathrm{~d}$ & $\bullet$ & $\bullet$ & $\bullet$ & $\bullet$ \\
\hline${ }^{46} \mathrm{Sc}_{m}$ & $18.75 \mathrm{~s}$ & $\bullet$ & $\bullet$ & $\bullet$ & $\bullet$ \\
\hline${ }^{47} \mathrm{Sc}$ & $3.3492 \mathrm{~d}$ & $\bullet$ & $\bullet$ & $\bullet$ & $\bullet$ \\
\hline${ }^{48} \mathrm{Sc}$ & $43.67 \mathrm{~h}$ & $\bullet$ & $\bullet$ & $\bullet$ & $\bullet$ \\
\hline${ }^{49} \mathrm{Sc}$ & $57.2 \mathrm{~m}$ & $\bullet$ & $\bullet$ & $\bullet$ & $\bullet$ \\
\hline${ }^{50} \mathrm{Sc}$ & $102.5 \mathrm{~s}$ & $\bullet$ & $\bullet$ & $\bullet$ & $\bullet$ \\
\hline${ }^{50} \mathrm{Sc}_{m}$ & $0.35 \mathrm{~s}$ & $\bullet$ & $\bullet$ & $\bullet$ & $\bullet$ \\
\hline${ }^{44} \mathrm{Ti}$ & $60.0 \mathrm{y}$ & $\bullet$ & & $\bullet$ & $\bullet$ \\
\hline
\end{tabular}


Table 6: (continued)

\begin{tabular}{|c|c|c|c|c|c|}
\hline$\overline{{ }^{A} Z}$ & lifetime & $\overline{(p, n)}$ & 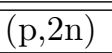 & $\overline{\overline{(d, n)}}$ & $(\mathrm{d}, 2 \mathrm{n})$ \\
\hline${ }^{45} \mathrm{Ti}$ & $184.8 \mathrm{~m}$ & $\bullet$ & & $\bullet$ & $\bullet$ \\
\hline${ }^{46} \mathrm{Ti}$ & $8.25 \%$ & - & & - & - \\
\hline${ }^{47} \mathrm{Ti}$ & $7.44 \%$ & - & - & • & $\bullet$ \\
\hline${ }^{48} \mathrm{Ti}$ & $73.72 \%$ & - & - & - & • \\
\hline${ }^{49} \mathrm{Ti}$ & $5.41 \%$ & - & - & - & • \\
\hline${ }^{50} \mathrm{Ti}$ & $5.18 \%$ & - & - & - & - \\
\hline${ }^{51} \mathrm{Ti}$ & $5.76 \mathrm{~m}$ & - & - & - & - \\
\hline${ }^{52} \mathrm{Ti}$ & $1.7 \mathrm{~m}$ & - & - & - & - \\
\hline${ }^{46} \mathrm{~V}$ & $422.50 \mathrm{~ms}$ & - & & - & - \\
\hline${ }^{46} \mathrm{~V}_{m}$ & $1 \mathrm{~ms}$ & - & & - & • \\
\hline${ }^{47} \mathrm{~V}$ & $32.6 \mathrm{~m}$ & - & & - & - \\
\hline${ }^{48} \mathrm{~V}$ & $15.9735 \mathrm{~d}$ & - & - & - & - \\
\hline${ }^{49} \mathrm{~V}$ & $330 \mathrm{~d}$ & - & - & - & - \\
\hline${ }^{50} \mathrm{~V}$ & $0.250 \%$ & - & - & - & - \\
\hline${ }^{51} \mathrm{~V}$ & $99.750 \%$ & - & - & - & - \\
\hline${ }^{52} \mathrm{~V}$ & $3.743 \mathrm{~m}$ & - & - & - & - \\
\hline${ }^{53} \mathrm{~V}$ & $1.60 \mathrm{~m}$ & - & - & - & - \\
\hline${ }^{48} \mathrm{Cr}$ & $21.56 \mathrm{~h}$ & - & & - & - \\
\hline${ }^{49} \mathrm{Cr}$ & $42.3 \mathrm{~m}$ & - & & - & - \\
\hline${ }^{50} \mathrm{Cr}$ & $4.345 \%$ & - & & $\bullet$ & $\bullet$ \\
\hline${ }^{51} \mathrm{Cr}$ & $27.7025 \mathrm{~d}$ & - & - & - & - \\
\hline${ }^{52} \mathrm{Cr}$ & $83.789 \%$ & - & $\bullet$ & $\bullet$ & • \\
\hline${ }^{53} \mathrm{Cr}$ & $9.501 \%$ & - & - & - & - \\
\hline${ }^{54} \mathrm{Cr}$ & $2.365 \%$ & $\bullet$ & - & - & - \\
\hline${ }^{55} \mathrm{Cr}$ & $3.497 \mathrm{~m}$ & - & - & - & - \\
\hline${ }^{56} \mathrm{Cr}$ & $5.94 \mathrm{~m}$ & - & - & - & - \\
\hline${ }^{50} \mathrm{Mn}$ & $283.29 \mathrm{~ms}$ & - & & - & - \\
\hline${ }^{50} \mathrm{Mn}_{m}$ & $1.75 \mathrm{~m}$ & - & & - & - \\
\hline${ }^{51} \mathrm{Mn}$ & $46.2 \mathrm{~m}$ & - & & - & - \\
\hline${ }^{52} \mathrm{Mn}$ & $5.591 \mathrm{~d}$ & - & - & - & - \\
\hline${ }^{52} \mathrm{Mn}_{m}$ & $21.1 \mathrm{~m}$ & - & • & • & • \\
\hline${ }^{53} \mathrm{Mn}$ & $3.74 \times 10^{6} \mathrm{y}$ & - & • & • & • \\
\hline${ }^{54} \mathrm{Mn}$ & $312.11 \mathrm{~d}$ & - & - & - & - \\
\hline${ }^{55} \mathrm{Mn}$ & $100 \%$ & - & - & - & - \\
\hline${ }^{56} \mathrm{Mn}$ & $2.5789 \mathrm{~h}$ & - & - & - & - \\
\hline${ }^{57} \mathrm{Mn}$ & $85.4 \mathrm{~s}$ & - & - & - & - \\
\hline${ }^{52} \mathrm{Fe}$ & $8.275 \mathrm{~h}$ & $\bullet$ & & $\bullet$ & $\bullet$ \\
\hline${ }^{53} \mathrm{Fe}$ & $8.51 \mathrm{~m}$ & - & & - & - \\
\hline${ }^{54} \mathrm{Fe}$ & $5.845 \%$ & - & & - & - \\
\hline${ }^{55} \mathrm{Fe}$ & $2.73 \mathrm{y}$ & - & - & • & - \\
\hline${ }^{56} \mathrm{Fe}$ & $91.754 \%$ & - & - & - & - \\
\hline${ }^{57} \mathrm{Fe}$ & $2.119 \%$ & - & - & - & - \\
\hline${ }^{58} \mathrm{Fe}$ & $0.282 \%$ & - & - & - & $\bullet$ \\
\hline
\end{tabular}




\section{A.2. Binding and Separation Energies}

Table 7:: Spins, parities, binding energies, and separation energies

\begin{tabular}{|c|c|c|c|c|c|c|}
\hline${ }^{A} Z$ & $\overline{J^{\pi}}$ & $\overline{\mathrm{BE}}(\mathrm{MeV})$ & $S_{n}(\mathrm{MeV})$ & $\overline{S_{p}(\mathrm{MeV})}$ & $\bar{S}_{\alpha}(\mathrm{MeV})$ & $S_{d}(\mathrm{MeV})$ \\
\hline${ }^{32} \mathrm{~S}$ & $0+$ & 271.78 & 15.04 & 8.86 & 6.95 & 18.95 \\
\hline${ }^{33} \mathrm{~S}$ & $3 / 2+$ & 280.42 & 8.64 & 9.57 & 7.11 & 15.28 \\
\hline${ }^{34} \mathrm{~S}$ & $0+$ & 291.84 & 11.42 & 10.88 & 7.92 & 18.76 \\
\hline${ }^{35} \mathrm{~S}$ & $3 / 2+$ & 298.82 & 6.99 & 11.58 & 8.32 & 15.64 \\
\hline${ }^{36} \mathrm{~S}$ & $0+$ & 308.71 & 9.89 & 13.10 & 9.01 & 19.24 \\
\hline${ }^{37} \mathrm{~S}$ & $7 / 2-$ & 313.02 & 4.30 & 13.93 & 8.83 & 15.17 \\
\hline${ }^{38} \mathrm{~S}$ & $0+$ & 321.05 & 8.04 & 15.16 & 9.33 & 19.75 \\
\hline${ }^{39} \mathrm{~S}$ & $3 / 2+$ & 325.43 & 4.37 & 15.69 & 11.23 & 17.30 \\
\hline${ }^{40} \mathrm{~S}$ & $0+$ & 333.20 & 7.78 & 17.28 & 12.81 & 21.24 \\
\hline${ }^{41} \mathrm{~S}$ & $5 / 2+$ & 337.43 & 4.22 & 18.20 & 14.86 & 19.28 \\
\hline${ }^{42} \mathrm{~S}$ & $0+$ & 344.16 & 6.73 & 19.69 & 16.03 & 22.71 \\
\hline${ }^{32} \mathrm{Cl}$ & $1+$ & 258.31 & 14.33 & 1.57 & 8.59 & 12.40 \\
\hline${ }^{33} \mathrm{Cl}$ & $3 / 2+$ & 274.06 & 15.74 & 2.28 & 6.47 & 15.09 \\
\hline${ }^{34} \mathrm{Cl}$ & $0+$ & 285.56 & 11.51 & 5.14 & 6.66 & 11.56 \\
\hline${ }^{34} \mathrm{Cl}_{m}$ & $3+$ & & & & & \\
\hline${ }^{35} \mathrm{Cl}$ & $3 / 2+$ & 298.21 & 12.64 & 6.37 & 7.00 & 15.56 \\
\hline${ }^{36} \mathrm{Cl}$ & $2+$ & 306.79 & 8.58 & 7.96 & 7.64 & 12.73 \\
\hline${ }^{37} \mathrm{Cl}$ & $3 / 2+$ & 317.10 & 10.31 & 8.39 & 7.85 & 16.05 \\
\hline${ }^{38} \mathrm{Cl}$ & $2-$ & 323.21 & 6.11 & 10.19 & 7.66 & 12.27 \\
\hline${ }^{38} \mathrm{Cl}_{m}$ & $5-$ & & & & & \\
\hline${ }^{39} \mathrm{Cl}$ & $3 / 2+$ & 331.28 & 8.07 & 10.23 & 7.37 & 16.04 \\
\hline${ }^{40} \mathrm{Cl}$ & $2-$ & 337.11 & 5.83 & 11.68 & 9.73 & 13.83 \\
\hline${ }^{41} \mathrm{Cl}$ & $1 / 2+$ & 344.93 & 7.82 & 11.73 & 10.74 & 17.28 \\
\hline${ }^{42} \mathrm{Cl}$ & $2+$ & 350.61 & 5.68 & 13.18 & 12.58 & 15.18 \\
\hline${ }^{43} \mathrm{Cl}$ & $1 / 2+$ & 357.93 & 7.33 & 13.78 & 13.72 & 18.28 \\
\hline${ }^{44} \mathrm{Cl}$ & $4+$ & 362.07 & 4.13 & 15.55 & 14.55 & 15.69 \\
\hline${ }^{45} \mathrm{Cl}$ & $3 / 2+$ & 368.27 & 6.20 & 16.54 & 15.51 & 19.53 \\
\hline${ }^{34} \mathrm{Ar}$ & $0+$ & 278.72 & 17.06 & 4.66 & 6.74 & 18.18 \\
\hline${ }^{35} \mathrm{Ar}$ & $3 / 2+$ & 291.46 & 12.74 & 5.90 & 6.43 & 15.18 \\
\hline${ }^{36} \mathrm{Ar}$ & $0+$ & 306.72 & 15.26 & 8.51 & 6.64 & 18.93 \\
\hline${ }^{37} \mathrm{Ar}$ & $3 / 2+$ & 315.50 & 8.79 & 8.71 & 6.79 & 15.07 \\
\hline${ }^{38} \mathrm{Ar}$ & $0+$ & 327.34 & 11.84 & 10.24 & 7.21 & 18.33 \\
\hline${ }^{39} \mathrm{Ar}$ & $7 / 2-$ & 333.94 & 6.60 & 10.73 & 6.82 & 14.62 \\
\hline${ }^{40} \mathrm{Ar}$ & $0+$ & 343.81 & 9.87 & 12.53 & 6.80 & 18.38 \\
\hline${ }^{41} \mathrm{Ar}$ & $7 / 2-$ & 349.91 & 6.10 & 12.80 & 8.59 & 16.40 \\
\hline${ }^{42} \mathrm{Ar}$ & $0+$ & 359.34 & 9.43 & 14.40 & 9.99 & 20.00 \\
\hline${ }^{43} \mathrm{Ar}$ & $3 / 2+$ & 364.99 & 5.66 & 14.39 & 11.27 & 17.84 \\
\hline${ }^{44} \mathrm{Ar}$ & $0+$ & 373.73 & 8.73 & 15.79 & 12.23 & 20.90 \\
\hline${ }^{45} \mathrm{Ar}$ & $1 / 2+$ & 378.90 & 5.17 & 16.83 & 13.18 & 18.74 \\
\hline${ }^{46} \mathrm{Ar}$ & $0+$ & 386.92 & 8.02 & 18.65 & 14.47 & 22.62 \\
\hline${ }^{47} \mathrm{Ar}$ & $3 / 2+$ & 391.18 & 4.26 & 18.49 & 16.37 & 20.68 \\
\hline${ }^{48} \mathrm{Ar}$ & $0+$ & 397.06 & 5.88 & 20.49 & 17.02 & 22.14 \\
\hline${ }^{35} \mathrm{~K}$ & $3 / 2+$ & 278.80 & 17.76 & 0.08 & 6.53 & 14.92 \\
\hline${ }^{36} \mathrm{~K}$ & $2+$ & 293.13 & 14.33 & 1.67 & 6.52 & 12.18 \\
\hline${ }^{37} \mathrm{~K}$ & $3 / 2+$ & 308.57 & 15.45 & 1.86 & 6.22 & 14.89 \\
\hline${ }^{38} \mathrm{~K}$ & $3+$ & 320.65 & 12.07 & 5.14 & 6.78 & 11.71 \\
\hline${ }^{38} \mathrm{~K}_{m}$ & $0+$ & & & & & \\
\hline${ }^{39} \mathrm{~K}$ & $3 / 2+$ & 333.72 & 13.08 & 6.38 & 7.22 & 16.00 \\
\hline${ }^{40} \mathrm{~K}$ & $4-$ & 341.52 & 7.80 & 7.58 & 6.44 & 11.96 \\
\hline
\end{tabular}


Table 7: (continued)

\begin{tabular}{|c|c|c|c|c|c|c|}
\hline${ }^{A} Z$ & $\overline{J^{\pi}}$ & $\overline{\mathrm{BEE}(\mathrm{MeV})}$ & $\overline{S_{n}(\mathrm{MeV})}$ & 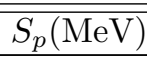 & $\overline{S_{\alpha}(\mathrm{MeV})}$ & $\overline{S_{d}(\mathrm{MeV})}$ \\
\hline${ }^{41} \mathrm{~K}$ & $3 / 2+$ & 351.62 & 10.10 & 7.81 & 6.22 & 15.45 \\
\hline${ }^{42} \mathrm{~K}$ & $2-$ & 359.15 & 7.53 & 9.24 & 7.65 & 13.12 \\
\hline${ }^{43} \mathrm{~K}$ & $3 / 2+$ & 368.80 & 9.64 & 9.46 & 9.22 & 16.66 \\
\hline${ }^{44} \mathrm{~K}$ & $2-$ & 376.08 & 7.29 & 11.09 & 10.68 & 14.52 \\
\hline${ }^{45} \mathrm{~K}$ & $3 / 2+$ & 384.95 & 8.87 & 11.22 & 11.72 & 17.73 \\
\hline${ }^{46} \mathrm{~K}$ & $2-$ & 391.83 & 6.88 & 12.94 & 12.93 & 15.88 \\
\hline${ }^{47} \mathrm{~K}$ & $1 / 2+$ & 400.18 & 8.35 & 13.27 & 13.95 & 19.06 \\
\hline${ }^{48} \mathrm{~K}$ & $2-$ & 404.68 & 4.50 & 13.51 & 14.32 & 15.54 \\
\hline${ }^{49} \mathrm{~K}$ & $3 / 2+$ & 410.95 & 6.27 & 13.89 & 14.38 & 17.55 \\
\hline${ }^{50} \mathrm{~K}$ & $0+$ & 414.05 & 3.10 & 14.50 & 13.07 & 14.77 \\
\hline${ }^{36} \mathrm{Ca}$ & $0+$ & 281.36 & 19.11 & 2.56 & 6.66 & 18.09 \\
\hline${ }^{37} \mathrm{Ca}$ & $3 / 2+$ & 296.15 & 14.79 & 3.02 & 6.20 & 15.13 \\
\hline${ }^{38} \mathrm{Ca}$ & $0+$ & 313.12 & 16.97 & 4.55 & 6.11 & 17.77 \\
\hline${ }^{39} \mathrm{Ca}$ & $3 / 2+$ & 326.41 & 13.29 & 5.76 & 6.65 & 15.61 \\
\hline${ }^{40} \mathrm{Ca}$ & $0+$ & 342.05 & 15.64 & 8.33 & 7.04 & 19.18 \\
\hline${ }^{41} \mathrm{Ca}$ & $7 / 2-$ & 350.41 & 8.36 & 8.89 & 6.61 & 14.47 \\
\hline${ }^{42} \mathrm{Ca}$ & $0+$ & 361.90 & 11.48 & 10.28 & 6.26 & 18.15 \\
\hline${ }^{43} \mathrm{Ca}$ & $7 / 2-$ & 369.83 & 7.93 & 10.68 & 7.59 & 15.99 \\
\hline${ }^{44} \mathrm{Ca}$ & $0+$ & 380.96 & 11.13 & 12.16 & 8.85 & 19.58 \\
\hline${ }^{45} \mathrm{Ca}$ & $7 / 2-$ & 388.37 & 7.41 & 12.29 & 10.17 & 17.35 \\
\hline${ }^{46} \mathrm{Ca}$ & $0+$ & 398.77 & 10.39 & 13.82 & 11.14 & 20.46 \\
\hline${ }^{47} \mathrm{Ca}$ & $7 / 2-$ & 406.05 & 7.28 & 14.21 & 12.75 & 18.87 \\
\hline${ }^{48} \mathrm{Ca}$ & $0+$ & 415.99 & 9.95 & 15.81 & 13.96 & 21.93 \\
\hline${ }^{49} \mathrm{Ca}$ & $3 / 2-$ & 421.14 & 5.15 & 16.45 & 13.94 & 18.73 \\
\hline${ }^{50} \mathrm{Ca}$ & $0+$ & 427.49 & 6.35 & 16.54 & 12.27 & 20.58 \\
\hline${ }^{51} \mathrm{Ca}$ & $3 / 2-$ & 431.85 & 4.36 & 17.80 & 12.38 & 18.68 \\
\hline${ }^{52} \mathrm{Ca}$ & $0+$ & 436.57 & 4.72 & 17.80 & 11.22 & 20.29 \\
\hline${ }^{38} \mathrm{Sc}$ & $2+$ & 295.22 & 15.85 & -0.94 & 5.88 & 11.63 \\
\hline${ }^{39} \mathrm{Sc}$ & $3 / 2+$ & 312.52 & 17.30 & -0.60 & 5.42 & 14.14 \\
\hline${ }^{40} \mathrm{Sc}$ & $4-$ & 326.95 & 14.43 & 0.54 & 5.52 & 11.60 \\
\hline${ }^{41} \mathrm{Sc}$ & $7 / 2-$ & 343.14 & 16.19 & 1.09 & 6.27 & 14.50 \\
\hline${ }^{42} \mathrm{Sc}$ & $0+$ & 354.69 & 11.55 & 4.27 & 5.74 & 10.41 \\
\hline${ }^{42} \mathrm{Sc}_{m}$ & $5+$ & & & & & \\
\hline${ }^{43} \mathrm{Sc}$ & $7 / 2$ & 366.83 & 12.14 & 4.93 & 4.80 & 14.19 \\
\hline${ }^{44} \mathrm{Sc}$ & $2+$ & 376.52 & 9.70 & 6.70 & 6.70 & 12.40 \\
\hline${ }^{44} \mathrm{Sc}_{m}$ & $6+$ & & & & & \\
\hline${ }^{45} \mathrm{Sc}$ & $7 / 2$ & 387.85 & 11.32 & 6.89 & 7.93 & 15.80 \\
\hline${ }^{45} \mathrm{Sc}_{m}$ & $3 / 2+$ & & & & & \\
\hline${ }^{46} \mathrm{Sc}$ & $4+$ & 396.61 & 8.76 & 8.23 & 9.16 & 13.42 \\
\hline${ }^{46} \mathrm{Sc}_{m}$ & $1-$ & & & & & \\
\hline${ }^{47} \mathrm{Sc}$ & $7 / 2-$ & 407.25 & 10.65 & 8.49 & 10.16 & 16.66 \\
\hline${ }^{48} \mathrm{Sc}$ & $6+$ & 415.49 & 8.24 & 9.44 & 11.11 & 14.50 \\
\hline${ }^{49} \mathrm{Sc}$ & $7 / 2-$ & 425.62 & 10.13 & 9.63 & 12.37 & 17.35 \\
\hline${ }^{50} \mathrm{Sc}$ & $5+$ & 431.67 & 6.06 & 10.54 & 11.54 & 13.46 \\
\hline${ }^{50} \mathrm{Sc}_{m}$ & $2+$ & & & & & \\
\hline${ }^{51} \mathrm{Sc}$ & $7 / 2-$ & 438.43 & 6.75 & 10.94 & 9.95 & 15.06 \\
\hline${ }^{52} \mathrm{Sc}$ & $3+$ & 443.64 & 5.21 & 11.78 & 10.66 & 13.92 \\
\hline${ }^{53} \mathrm{Sc}$ & $7 / 2+$ & 448.97 & 5.34 & 12.40 & 9.73 & 14.90 \\
\hline${ }^{54} \mathrm{Sc}$ & $1+$ & 453.64 & 4.67 & 13.61 & 11.29 & 14.85 \\
\hline${ }^{39} \mathrm{Ti}$ & $1 / 2+$ & 296.07 & 15.67 & 0.85 & 5.53 & 14.48 \\
\hline${ }^{40} \mathrm{Ti}$ & $0+$ & 314.49 & 18.42 & 1.97 & 4.83 & 17.05 \\
\hline
\end{tabular}


Table 7: (continued)

\begin{tabular}{|c|c|c|c|c|c|c|}
\hline${ }^{A} Z$ & $\overline{J^{\pi}}$ & 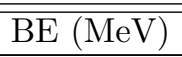 & $\overline{S_{n}(\mathrm{MeV})}$ & $\overline{S_{p}(\mathrm{MeV})}$ & $\overline{S_{\alpha}(\mathrm{MeV})}$ & $\overline{S_{d}(\mathrm{MeV})}$ \\
\hline${ }^{41} \mathrm{Ti}$ & $3 / 2+$ & 329.41 & 14.92 & 2.47 & 4.96 & 14.67 \\
\hline${ }^{42} \mathrm{Ti}$ & $0+$ & 346.91 & 17.49 & 3.77 & 5.49 & 17.73 \\
\hline${ }^{43} \mathrm{Ti}$ & $7 / 2-$ & 359.18 & 12.27 & 4.49 & 4.47 & 13.81 \\
\hline${ }^{44} \mathrm{Ti}$ & $0+$ & 375.47 & 16.30 & 8.65 & 5.13 & 18.56 \\
\hline${ }^{45} \mathrm{Ti}$ & $7 / 2-$ & 385.00 & 9.53 & 8.48 & 6.29 & 15.95 \\
\hline${ }^{46} \mathrm{Ti}$ & $0+$ & 398.19 & 13.19 & 10.34 & 8.00 & 19.44 \\
\hline${ }^{47} \mathrm{Ti}$ & $5 / 2-$ & 407.07 & 8.88 & 10.46 & 8.95 & 17.00 \\
\hline${ }^{48} \mathrm{Ti}$ & $0+$ & 418.70 & 11.63 & 11.44 & 9.44 & 19.87 \\
\hline${ }^{49} \mathrm{Ti}$ & $7 / 2-$ & 426.84 & 8.14 & 11.35 & 10.17 & 17.36 \\
\hline${ }^{50} \mathrm{Ti}$ & $0+$ & 437.78 & 10.94 & 12.16 & 10.72 & 20.07 \\
\hline${ }^{51} \mathrm{Ti}$ & $3 / 2-$ & 444.15 & 6.37 & 12.48 & 9.81 & 16.31 \\
\hline${ }^{52} \mathrm{Ti}$ & $0+$ & 451.96 & 7.81 & 13.54 & 7.67 & 18.06 \\
\hline${ }^{53} \mathrm{Ti}$ & $3 / 2-$ & 457.40 & 5.44 & 13.76 & 7.96 & 16.75 \\
\hline${ }^{54} \mathrm{Ti}$ & $0+$ & 464.23 & 6.84 & 15.26 & 8.45 & 18.37 \\
\hline${ }^{55} \mathrm{Ti}$ & $1 / 2+$ & 468.38 & 4.15 & 14.74 & 8.23 & 17.18 \\
\hline${ }^{56} \mathrm{Ti}$ & $0+$ & 473.72 & 5.34 & 16.65 & 8.85 & 17.85 \\
\hline${ }^{42} \mathrm{~V}$ & $2+$ & 329.17 & 16.04 & -0.24 & 5.66 & 12.45 \\
\hline${ }^{43} \mathrm{~V}$ & $1 / 2-$ & 347.10 & 17.93 & 0.19 & 6.28 & 15.46 \\
\hline${ }^{44} \mathrm{~V}$ & $3+$ & 361.26 & 14.16 & 2.08 & 6.02 & 12.13 \\
\hline${ }^{44} \mathrm{~V}_{m}$ & $6+$ & & & & & \\
\hline${ }^{45} \mathrm{~V}$ & $7 / 2-$ & 377.09 & 15.83 & 1.62 & 5.66 & 15.69 \\
\hline${ }^{46} \mathrm{~V}$ & $0+$ & 390.36 & 13.26 & 5.36 & 7.38 & 12.66 \\
\hline${ }^{46} \mathrm{~V}_{m}$ & $3+$ & & & & & \\
\hline${ }^{47} \mathrm{~V}$ & $3 / 2-$ & 403.36 & 13.00 & 5.17 & 8.24 & 16.13 \\
\hline${ }^{48} \mathrm{~V}$ & $4+$ & 413.90 & 10.54 & 6.83 & 9.08 & 13.49 \\
\hline${ }^{49} \mathrm{~V}$ & $7 / 2-$ & 425.46 & 11.55 & 6.76 & 9.31 & 16.16 \\
\hline${ }^{50} \mathrm{~V}$ & $6+$ & 434.79 & 9.34 & 7.95 & 9.89 & 13.87 \\
\hline${ }^{51} \mathrm{~V}$ & $7 / 2-$ & 445.84 & 11.05 & 8.06 & 10.29 & 16.78 \\
\hline${ }^{52} \mathrm{~V}$ & $3+$ & 453.16 & 7.31 & 9.00 & 9.37 & 13.15 \\
\hline${ }^{53} \mathrm{~V}$ & $7 / 2-$ & 461.63 & 8.48 & 9.67 & 7.72 & 15.26 \\
\hline${ }^{54} \mathrm{~V}$ & $3+$ & 467.75 & 6.11 & 10.35 & 7.78 & 13.56 \\
\hline${ }^{55} \mathrm{~V}$ & $7 / 2-$ & 475.08 & 7.33 & 10.85 & 8.36 & 15.46 \\
\hline${ }^{56} \mathrm{~V}$ & $2+$ & 480.08 & 5.00 & 11.70 & 8.15 & 13.62 \\
\hline${ }^{57} \mathrm{~V}$ & $3 / 2+$ & 486.26 & 6.18 & 12.54 & 8.99 & 15.65 \\
\hline${ }^{44} \mathrm{Cr}$ & $0+$ & 349.82 & 19.40 & 2.73 & 7.03 & 18.43 \\
\hline${ }^{45} \mathrm{Cr}$ & $5 / 2+$ & 363.40 & 13.58 & 2.14 & 5.69 & 14.08 \\
\hline${ }^{46} \mathrm{Cr}$ & $0+$ & 381.98 & 18.58 & 4.88 & 6.78 & 18.49 \\
\hline${ }^{47} \mathrm{Cr}$ & $3 / 2-$ & 395.13 & 13.16 & 4.77 & 7.66 & 15.81 \\
\hline${ }^{48} \mathrm{Cr}$ & $0+$ & 411.47 & 16.33 & 8.11 & 7.69 & 18.88 \\
\hline${ }^{49} \mathrm{Cr}$ & $5 / 2-$ & 422.05 & 10.58 & 8.14 & 8.75 & 16.46 \\
\hline${ }^{50} \mathrm{Cr}$ & $0+$ & 435.05 & 13.00 & 9.59 & 8.56 & 18.92 \\
\hline${ }^{51} \mathrm{Cr}$ & $7 / 2-$ & 444.31 & 9.26 & 9.52 & 8.94 & 16.63 \\
\hline${ }^{52} \mathrm{Cr}$ & $0+$ & 456.35 & 12.04 & 10.50 & 9.35 & 19.33 \\
\hline${ }^{53} \mathrm{Cr}$ & $3 / 2-$ & 464.29 & 7.94 & 11.13 & 9.15 & 16.22 \\
\hline${ }^{54} \mathrm{Cr}$ & $0+$ & 474.01 & 9.72 & 12.37 & 7.93 & 18.63 \\
\hline${ }^{55} \mathrm{Cr}$ & $3 / 2-$ & 480.25 & 6.25 & 12.51 & 7.80 & 16.39 \\
\hline${ }^{56} \mathrm{Cr}$ & $0+$ & 488.50 & 8.25 & 13.42 & 8.24 & 18.53 \\
\hline${ }^{57} \mathrm{Cr}$ & $3 / 2-$ & 493.81 & 5.31 & 13.73 & 8.12 & 16.51 \\
\hline${ }^{58} \mathrm{Cr}$ & $0+$ & 501.19 & 7.38 & 14.93 & 8.66 & 18.89 \\
\hline${ }^{46} \mathrm{Mn}$ & $4+$ & 364.09 & 15.33 & 0.69 & 6.62 & 12.04 \\
\hline${ }^{47} \mathrm{Mn}$ & $5 / 2+$ & 382.06 & 17.96 & 0.08 & 6.66 & 16.43 \\
\hline
\end{tabular}


Table 7: (continued)

\begin{tabular}{|c|c|c|c|c|c|c|}
\hline${ }^{A} Z$ & $\overline{J^{\pi}}$ & 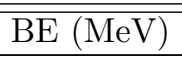 & $\overline{S_{n}(\mathrm{MeV})}$ & $\overline{S_{p}(\mathrm{MeV})}$ & $\overline{S_{\alpha}(\mathrm{MeV})}$ & $\overline{S_{d}(\mathrm{MeV})}$ \\
\hline${ }^{48} \mathrm{Mn}$ & $4+$ & 397.19 & 15.13 & 2.05 & 7.63 & 12.99 \\
\hline${ }^{49} \mathrm{Mn}$ & $5 / 2-$ & 413.55 & 16.36 & 2.09 & 8.16 & 16.19 \\
\hline${ }^{50} \mathrm{Mn}$ & $0+$ & 426.63 & 13.08 & 4.59 & 7.98 & 12.94 \\
\hline${ }^{50} \mathrm{Mn}_{m}$ & $5+$ & & & & & \\
\hline${ }^{51} \mathrm{Mn}$ & $5 / 2-$ & 440.32 & 13.69 & 5.27 & 8.66 & 16.05 \\
\hline${ }^{52} \mathrm{Mn}$ & $6+$ & 450.86 & 10.54 & 6.55 & 8.65 & 13.58 \\
\hline${ }^{52} \mathrm{Mn}_{m}$ & $2+$ & & & & & \\
\hline${ }^{53} \mathrm{Mn}$ & $7 / 2-$ & 462.91 & 12.05 & 6.56 & 9.15 & 16.37 \\
\hline${ }^{54} \mathrm{Mn}$ & $3+$ & 471.85 & 8.94 & 7.56 & 8.76 & 13.27 \\
\hline${ }^{55} \mathrm{Mn}$ & $5 / 2-$ & 482.07 & 10.23 & 8.07 & 7.93 & 15.56 \\
\hline${ }^{56} \mathrm{Mn}$ & $3+$ & 489.34 & 7.27 & 9.09 & 7.89 & 13.11 \\
\hline${ }^{57} \mathrm{Mn}$ & $5 / 2-$ & 497.99 & 8.65 & 9.49 & 8.06 & 15.52 \\
\hline${ }^{58} \mathrm{Mn}$ & $3+$ & 504.48 & 6.49 & 10.67 & 8.44 & 13.76 \\
\hline${ }^{58} \mathrm{Mn}_{m}$ & $4+$ & & & & & \\
\hline${ }^{59} \mathrm{Mn}$ & $3 / 2-$ & 512.13 & 7.64 & 10.93 & 8.75 & 16.09 \\
\hline${ }^{48} \mathrm{Fe}$ & $0+$ & 385.24 & 19.61 & 3.19 & 7.12 & 18.93 \\
\hline${ }^{49} \mathrm{Fe}$ & $7 / 2-$ & 399.74 & 14.49 & 2.55 & 8.04 & 15.45 \\
\hline${ }^{50} \mathrm{Fe}$ & $0+$ & 417.70 & 17.96 & 4.15 & 7.43 & 18.29 \\
\hline${ }^{51} \mathrm{Fe}$ & $5 / 2-$ & 431.52 & 13.82 & 4.88 & 8.09 & 15.74 \\
\hline${ }^{52} \mathrm{Fe}$ & $0+$ & 447.70 & 16.18 & 7.38 & 7.94 & 18.84 \\
\hline${ }^{53} \mathrm{Fe}$ & $7 / 2-$ & 458.38 & 10.68 & 7.53 & 8.04 & 15.84 \\
\hline${ }^{54} \mathrm{Fe}$ & $0+$ & 471.76 & 13.38 & 8.85 & 8.42 & 18.68 \\
\hline${ }^{55} \mathrm{Fe}$ & $3 / 2-$ & 481.06 & 9.30 & 9.21 & 8.45 & 15.93 \\
\hline${ }^{56} \mathrm{Fe}$ & $0+$ & 492.26 & 11.20 & 10.18 & 7.61 & 18.19 \\
\hline${ }^{57} \mathrm{Fe}$ & $1 / 2-$ & 499.90 & 7.65 & 10.56 & 7.32 & 15.61 \\
\hline${ }^{58} \mathrm{Fe}$ & $0+$ & 509.95 & 10.04 & 11.96 & 7.64 & 18.38 \\
\hline${ }^{59} \mathrm{Fe}$ & $3 / 2-$ & 516.53 & 6.58 & 12.05 & 7.98 & 16.31 \\
\hline${ }^{60} \mathrm{Fe}$ & $0+$ & 525.35 & 8.82 & 13.22 & 8.55 & 18.64 \\
\hline${ }^{50} \mathrm{Co}$ & $4+$ & 399.64 & 15.69 & -0.10 & 7.25 & 12.17 \\
\hline${ }^{51} \mathrm{Co}$ & $7 / 2+$ & 417.79 & 18.15 & 0.09 & 7.43 & 15.83 \\
\hline${ }^{52} \mathrm{Co}$ & $1+$ & 432.50 & 14.71 & 0.98 & 7.02 & 12.58 \\
\hline${ }^{53} \mathrm{Co}$ & $7 / 2-$ & 449.30 & 16.80 & 1.60 & 7.45 & 15.56 \\
\hline${ }^{53} \mathrm{Co}_{m}$ & $19 / 2-$ & & & & & \\
\hline${ }^{54} \mathrm{Co}$ & $0+$ & 462.74 & 13.44 & 4.35 & 7.81 & 12.81 \\
\hline${ }^{54} \mathrm{Co}_{m}$ & $7+$ & & & & & \\
\hline${ }^{55} \mathrm{Co}$ & $7 / 2-$ & 476.83 & 14.09 & 5.06 & 8.21 & 16.22 \\
\hline${ }^{56} \mathrm{Co}$ & $4+$ & 486.91 & 10.08 & 5.85 & 7.76 & 12.92 \\
\hline${ }^{57} \mathrm{Co}$ & $7 / 2-$ & 498.29 & 11.38 & 6.03 & 7.08 & 15.00 \\
\hline${ }^{58} \mathrm{Co}$ & $2+$ & 506.86 & 8.57 & 6.95 & 6.71 & 12.38 \\
\hline${ }^{58} \mathrm{Co}_{m} 1$ & $5+$ & & & & & \\
\hline${ }^{58} \mathrm{Co}_{m} 2$ & $4+$ & & & & & \\
\hline${ }^{59} \mathrm{Co}$ & $7 / 2-$ & 517.31 & 10.45 & 7.36 & 6.94 & 15.18 \\
\hline${ }^{60} \mathrm{Co}$ & $5+$ & 524.80 & 7.49 & 8.27 & 7.16 & 12.63 \\
\hline${ }^{60} \mathrm{Co}_{m}$ & $2+$ & & & & & \\
\hline
\end{tabular}




\section{A.3. Q-values for Select Reactions}

Table 8:: Q-values $(\mathrm{MeV})$ for select reactions

\begin{tabular}{|c|c|c|c|c|c|c|c|c|c|c|}
\hline Target & $(\mathrm{n}, 2 \mathrm{n})$ & $(n, 3 n)$ & $(\mathrm{n}, \mathrm{p})$ & $(\mathrm{n}, \mathrm{np})$ & $(\mathrm{n}, \alpha)$ & $(\mathrm{n}, \mathrm{d})$ & $(\mathrm{p}, \mathrm{n})$ & $(\mathrm{p}, 2 \mathrm{n})$ & $(\mathrm{d}, \mathrm{n})$ & $(\mathrm{d}, 2 \mathrm{n})$ \\
\hline${ }^{41} \mathrm{Ca}$ & -8.36 & -24.01 & 1.20 & -8.89 & 5.22 & -6.67 & -7.28 & -23.47 & 2.05 & -9.50 \\
\hline${ }^{42} \mathrm{Ca}$ & -11.48 & -19.84 & -2.74 & -10.28 & 0.34 & -8.05 & -7.21 & -18.76 & 2.71 & -9.43 \\
\hline${ }^{43} \mathrm{Ca}$ & -7.93 & -19.41 & -1.03 & -10.68 & 2.28 & -8.45 & -3.00 & -15.14 & 4.47 & -5.23 \\
\hline${ }^{44} \mathrm{Ca}$ & -11.13 & -19.06 & -4.88 & -12.16 & -2.75 & -9.94 & -4.43 & -14.13 & 4.66 & -6.66 \\
\hline${ }^{45} \mathrm{Ca}$ & -7.41 & -18.55 & -3.42 & -12.29 & -0.74 & -10.07 & -0.53 & -11.85 & 6.01 & -2.75 \\
\hline${ }^{46} \mathrm{Ca}$ & -10.39 & -17.81 & -6.93 & -13.82 & -5.48 & -11.59 & -2.16 & -10.92 & 6.26 & -4.38 \\
\hline${ }^{47} \mathrm{Ca}$ & -7.28 & -17.67 & -5.86 & -14.21 & -4.02 & -11.99 & 1.21 & -9.44 & 7.22 & -1.02 \\
\hline${ }^{48} \mathrm{Ca}$ & -9.95 & -17.22 & -11.31 & -15.81 & -8.80 & -13.58 & -0.50 & -8.74 & 7.40 & -2.72 \\
\hline${ }^{41} \mathrm{Sc}$ & -16.19 & -30.62 & 7.28 & -1.09 & 5.80 & 1.14 & -13.72 & -28.65 & 1.54 & -15.95 \\
\hline${ }^{42} \mathrm{Sc}$ & -11.55 & -27.74 & 7.21 & -4.27 & 7.33 & -2.05 & -7.78 & -25.27 & 2.26 & -10.01 \\
\hline${ }^{43} \mathrm{Sc}$ & -12.14 & -23.69 & 3.00 & -4.93 & 2.99 & -2.71 & -7.65 & -19.92 & 6.42 & -9.87 \\
\hline${ }^{44} \mathrm{Sc}$ & -9.70 & -21.84 & 4.43 & -6.70 & 3.39 & -4.47 & -1.05 & -17.35 & 6.25 & -3.27 \\
\hline${ }^{45} \mathrm{Sc}$ & -11.32 & -21.02 & 0.53 & -6.89 & -0.40 & -4.66 & -2.84 & -12.37 & 8.12 & -5.07 \\
\hline${ }^{46} \mathrm{Sc}$ & -8.76 & -20.08 & 2.16 & -8.23 & 0.48 & -6.01 & 1.58 & -11.61 & 8.24 & -0.64 \\
\hline${ }^{47} \mathrm{Sc}$ & -10.65 & -19.41 & -1.21 & -8.49 & -2.88 & -6.26 & -0.18 & -9.06 & 9.22 & -2.41 \\
\hline${ }^{48} \mathrm{Sc}$ & -8.24 & -18.88 & 0.50 & -9.44 & -2.24 & -7.22 & 3.21 & -8.42 & 9.13 & 0.98 \\
\hline${ }^{49} \mathrm{Sc}$ & -10.13 & -18.36 & -4.48 & -9.63 & -5.49 & -7.40 & 1.22 & -6.92 & 9.94 & -1.00 \\
\hline${ }^{50} \mathrm{Sc}$ & -6.06 & -16.18 & -4.18 & -10.54 & -3.19 & -8.31 & 6.11 & -4.83 & 10.26 & 3.88 \\
\hline${ }^{44} \mathrm{Ti}$ & -16.30 & -28.57 & 1.05 & -8.65 & 3.24 & -6.42 & -14.21 & -28.38 & -0.60 & -16.44 \\
\hline${ }^{45} \mathrm{Ti}$ & -9.53 & -25.83 & 2.84 & -8.48 & 5.19 & -6.25 & -7.91 & -23.74 & 3.13 & -10.13 \\
\hline${ }^{46} \mathrm{Ti}$ & -13.19 & -22.72 & -1.58 & -10.34 & -0.07 & -8.12 & -7.83 & -21.10 & 2.94 & -10.06 \\
\hline${ }^{47} \mathrm{Ti}$ & -8.88 & -22.07 & 0.18 & -10.46 & 2.18 & -8.24 & -3.71 & -16.71 & 4.61 & -5.94 \\
\hline${ }^{48} \mathrm{Ti}$ & -11.63 & -20.51 & -3.21 & -11.44 & -2.03 & -9.22 & -4.79 & -15.34 & 4.53 & -7.02 \\
\hline${ }^{49} \mathrm{Ti}$ & -8.14 & -19.77 & -1.22 & -11.35 & 0.22 & -9.13 & -1.38 & -12.94 & 5.73 & -3.61 \\
\hline${ }^{50} \mathrm{Ti}$ & -10.94 & -19.08 & -6.11 & -12.16 & -3.44 & -9.94 & -2.99 & -12.32 & 5.84 & -5.21 \\
\hline${ }^{51} \mathrm{Ti}$ & -6.37 & -17.31 & -5.73 & -12.48 & 0.13 & -10.26 & 1.69 & -9.36 & 6.78 & -0.53 \\
\hline${ }^{52} \mathrm{Ti}$ & -7.81 & -14.18 & -8.33 & -13.54 & -2.53 & -11.31 & 1.19 & -6.12 & 7.45 & -1.03 \\
\hline${ }^{46} \mathrm{~V}$ & -13.26 & -29.10 & 7.83 & -5.36 & 4.76 & -3.13 & -8.38 & -26.96 & 2.55 & -10.61 \\
\hline${ }^{47} \mathrm{~V}$ & -13.00 & -26.27 & 3.71 & -5.17 & 1.46 & -2.94 & -8.23 & -21.38 & 5.88 & -10.45 \\
\hline${ }^{48} \mathrm{~V}$ & -10.54 & -23.55 & 4.79 & -6.83 & 2.24 & -4.61 & -2.44 & -18.77 & 5.92 & -4.66 \\
\hline${ }^{49} \mathrm{~V}$ & -11.55 & -22.10 & 1.38 & -6.76 & -0.55 & -4.53 & -3.41 & -13.99 & 7.37 & -5.63 \\
\hline${ }^{50} \mathrm{~V}$ & -9.34 & -20.89 & 2.99 & -7.95 & 0.76 & -5.73 & 0.26 & -12.74 & 7.29 & -1.97 \\
\hline${ }^{51} \mathrm{~V}$ & -11.05 & -20.39 & -1.69 & -8.06 & -2.06 & -5.84 & -1.53 & -10.80 & 8.28 & -3.76 \\
\hline${ }^{52} \mathrm{~V}$ & -7.31 & -18.36 & -1.19 & -9.00 & 0.76 & -6.78 & 3.19 & -8.85 & 8.91 & 0.97 \\
\hline${ }^{53} \mathrm{~V}$ & -8.48 & -15.79 & -4.24 & -9.67 & -1.67 & -7.45 & 2.65 & -5.29 & 10.15 & 0.43 \\
\hline${ }^{48} \mathrm{Cr}$ & -16.33 & -29.49 & 2.44 & -8.11 & 1.83 & -5.88 & -14.28 & -29.41 & -0.14 & -16.50 \\
\hline${ }^{49} \mathrm{Cr}$ & -10.58 & -26.91 & 3.41 & -8.14 & 4.44 & -5.92 & -8.50 & -24.86 & 2.36 & -10.72 \\
\hline${ }^{50} \mathrm{Cr}$ & -13.00 & -23.58 & -0.26 & -9.59 & 0.32 & -7.37 & -8.42 & -21.50 & 3.05 & -10.64 \\
\hline${ }^{51} \mathrm{Cr}$ & -9.26 & -22.26 & 1.53 & -9.52 & 2.69 & -7.29 & -3.99 & -17.68 & 4.32 & -6.21 \\
\hline${ }^{52} \mathrm{Cr}$ & -12.04 & -21.30 & -3.19 & -10.50 & -1.21 & -8.28 & -5.49 & -16.03 & 4.34 & -7.72 \\
\hline${ }^{53} \mathrm{Cr}$ & -7.94 & -19.98 & -2.65 & -11.13 & 1.79 & -8.91 & -1.38 & -13.43 & 5.34 & -3.60 \\
\hline${ }^{54} \mathrm{Cr}$ & -9.72 & -17.66 & -6.26 & -12.37 & -1.56 & -10.15 & -2.16 & -11.10 & 5.84 & -4.38 \\
\hline${ }^{55} \mathrm{Cr}$ & -6.25 & -15.97 & -5.17 & -12.51 & 0.00 & -10.28 & 1.82 & -8.41 & 6.87 & -0.40 \\
\hline${ }^{56} \mathrm{Cr}$ & -8.25 & -14.49 & -8.42 & -13.42 & -2.81 & -11.19 & 0.85 & -6.42 & 7.27 & -1.38 \\
\hline${ }^{50} \mathrm{Mn}$ & -13.08 & -29.45 & 8.42 & -4.59 & 5.02 & -2.36 & -8.93 & -26.90 & 2.66 & -11.16 \\
\hline${ }^{51} \mathrm{Mn}$ & -13.69 & -26.77 & 3.99 & -5.27 & 1.88 & -3.05 & -8.80 & -22.62 & 5.15 & -11.03 \\
\hline${ }^{52} \mathrm{Mn}$ & -10.54 & -24.22 & 5.49 & -6.55 & 2.90 & -4.32 & -3.16 & -19.34 & 5.30 & -5.38 \\
\hline${ }^{53} \mathrm{Mn}$ & -12.05 & -22.59 & 1.38 & -6.56 & 0.18 & -4.34 & -4.52 & -15.21 & 6.63 & -6.75 \\
\hline${ }^{54} \mathrm{Mn}$ & -8.94 & -20.99 & 2.16 & -7.56 & 2.29 & -5.33 & -0.09 & -13.46 & 6.99 & -2.31 \\
\hline
\end{tabular}


Table 8: (continued)

\begin{tabular}{lrrrrrrrrrr}
\hline \hline Target & $(\mathrm{n}, 2 \mathrm{n})$ & $(\mathrm{n}, 3 \mathrm{n})$ & $(\mathrm{n}, \mathrm{p})$ & $(\mathrm{n}, \mathrm{np})$ & $(\mathrm{n}, \alpha)$ & $(\mathrm{n}, \mathrm{d})$ & $(\mathrm{p}, \mathrm{n})$ & $(\mathrm{p}, 2 \mathrm{n})$ & $(\mathrm{d}, \mathrm{n})$ & $(\mathrm{d}, 2 \mathrm{n})$ \\
\hline${ }^{55} \mathrm{Mn}$ & -10.23 & -19.17 & -1.82 & -8.07 & -0.62 & -5.84 & -1.01 & -10.31 & 7.96 & -3.24 \\
${ }^{56} \mathrm{Mn}$ & -7.27 & -17.50 & -0.85 & -9.09 & 0.59 & -6.87 & 2.91 & -8.28 & 8.33 & 0.69 \\
${ }^{57} \mathrm{Mn}$ & -8.65 & -15.92 & -4.18 & -9.49 & -1.95 & -7.27 & 1.91 & -5.74 & 9.73 & -0.31 \\
\hline${ }^{52} \mathrm{Fe}$ & -16.18 & -30.00 & 3.16 & -7.38 & 2.65 & -5.15 & -15.20 & -29.91 & -0.62 & -17.42 \\
${ }^{53} \mathrm{Fe}$ & -10.68 & -26.87 & 4.52 & -7.53 & 4.96 & -5.30 & -9.08 & -25.88 & 2.13 & -11.31 \\
${ }^{54} \mathrm{Fe}$ & -13.38 & -24.06 & 0.09 & -8.85 & 0.84 & -6.63 & -9.03 & -22.46 & 2.84 & -11.25 \\
${ }^{55} \mathrm{Fe}$ & -9.30 & -22.68 & 1.01 & -9.21 & 3.58 & -6.99 & -4.23 & -18.32 & 3.62 & -6.46 \\
${ }^{56} \mathrm{Fe}$ & -11.20 & -20.50 & -2.91 & -10.18 & 0.33 & -7.96 & -5.35 & -15.43 & 3.80 & -7.57 \\
${ }^{57} \mathrm{Fe}$ & -7.65 & -18.84 & -1.91 & -10.56 & 2.40 & -8.33 & -1.62 & -12.99 & 4.73 & -3.84 \\
${ }^{58} \mathrm{Fe}$ & -10.04 & -17.69 & -5.46 & -11.96 & -1.40 & -9.73 & -3.09 & -11.66 & 5.14 & -5.31 \\
\hline
\end{tabular}




\section{A.4. Adopted Level Scheme Diagrams}

Here we present discrete level scheme diagrams that cover the range of nuclei presented in the production and destruction cross section plots. Many have been modified from their orignial versions in RIPL. Only the first 25 levels are included for most isotopes. For ${ }^{47,49,52} \mathrm{~V}$, only 20 levels are shown.
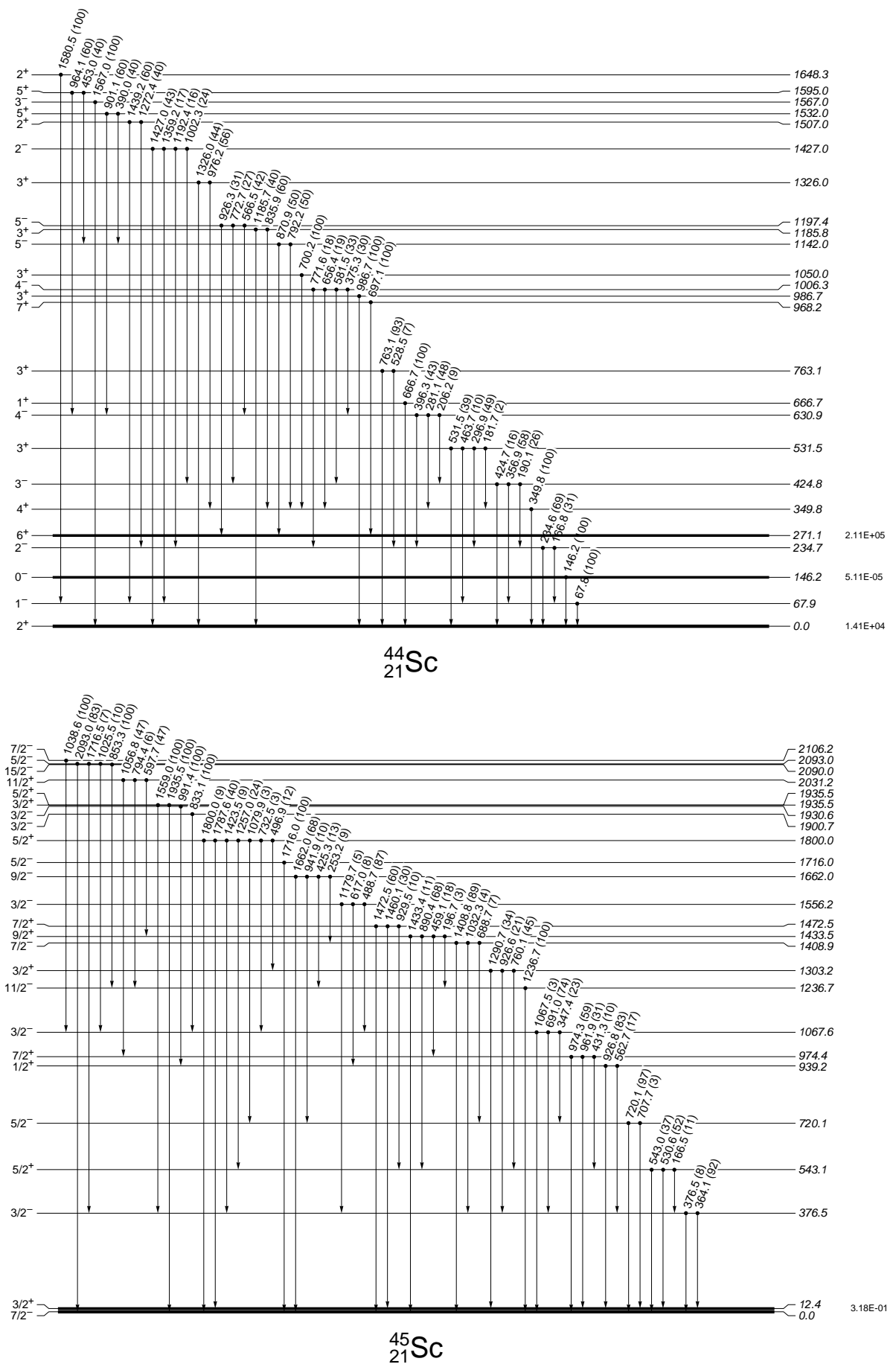

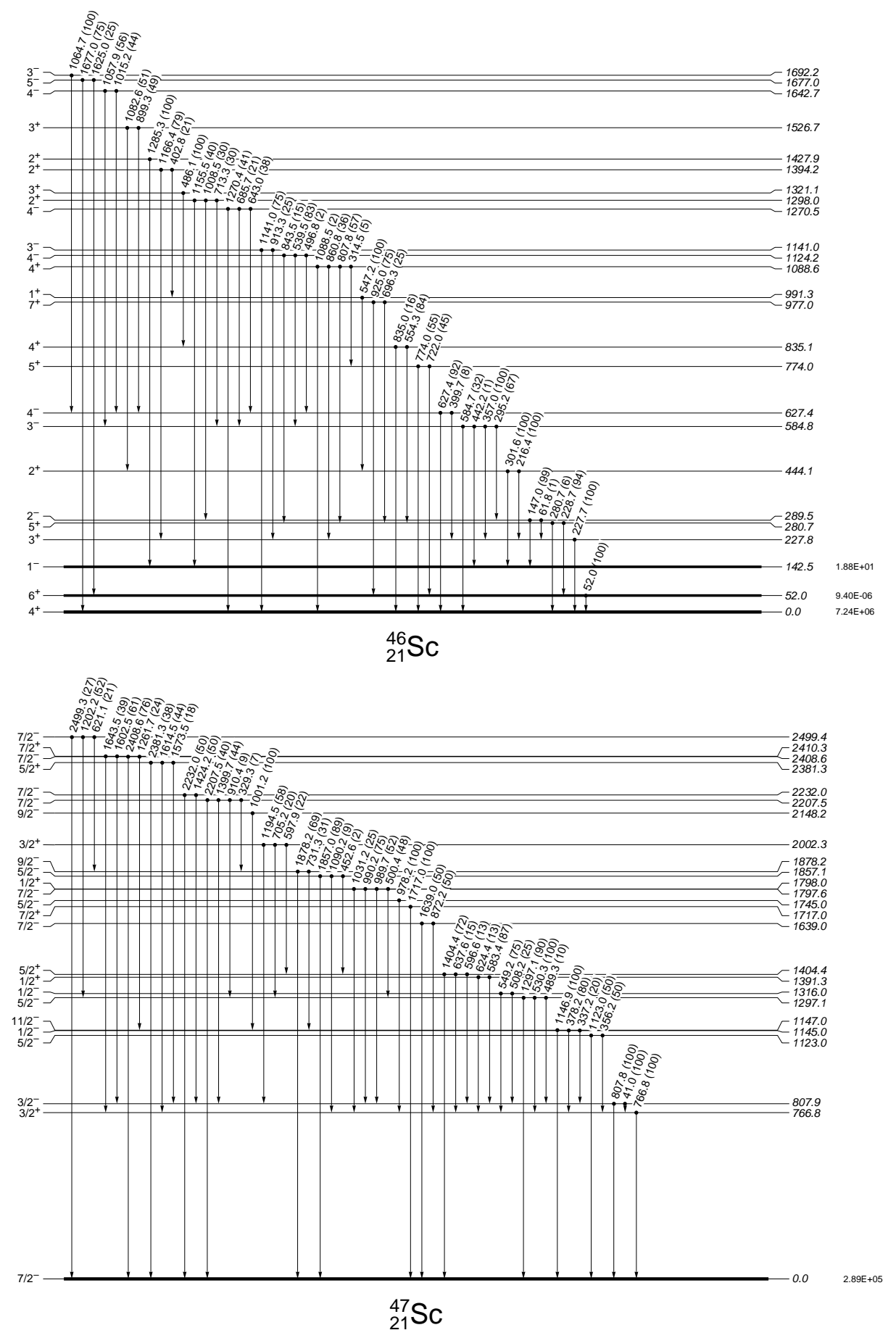

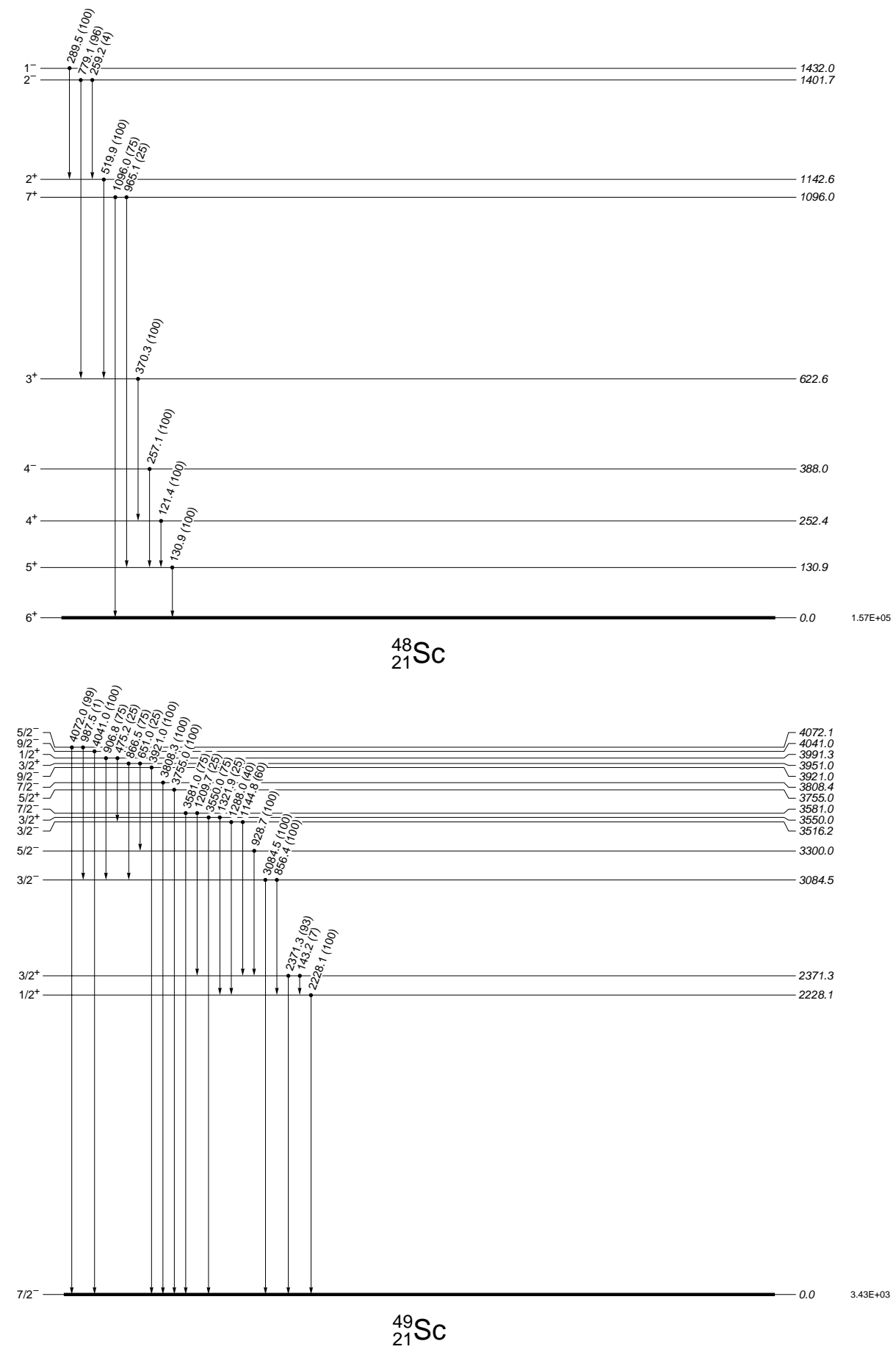

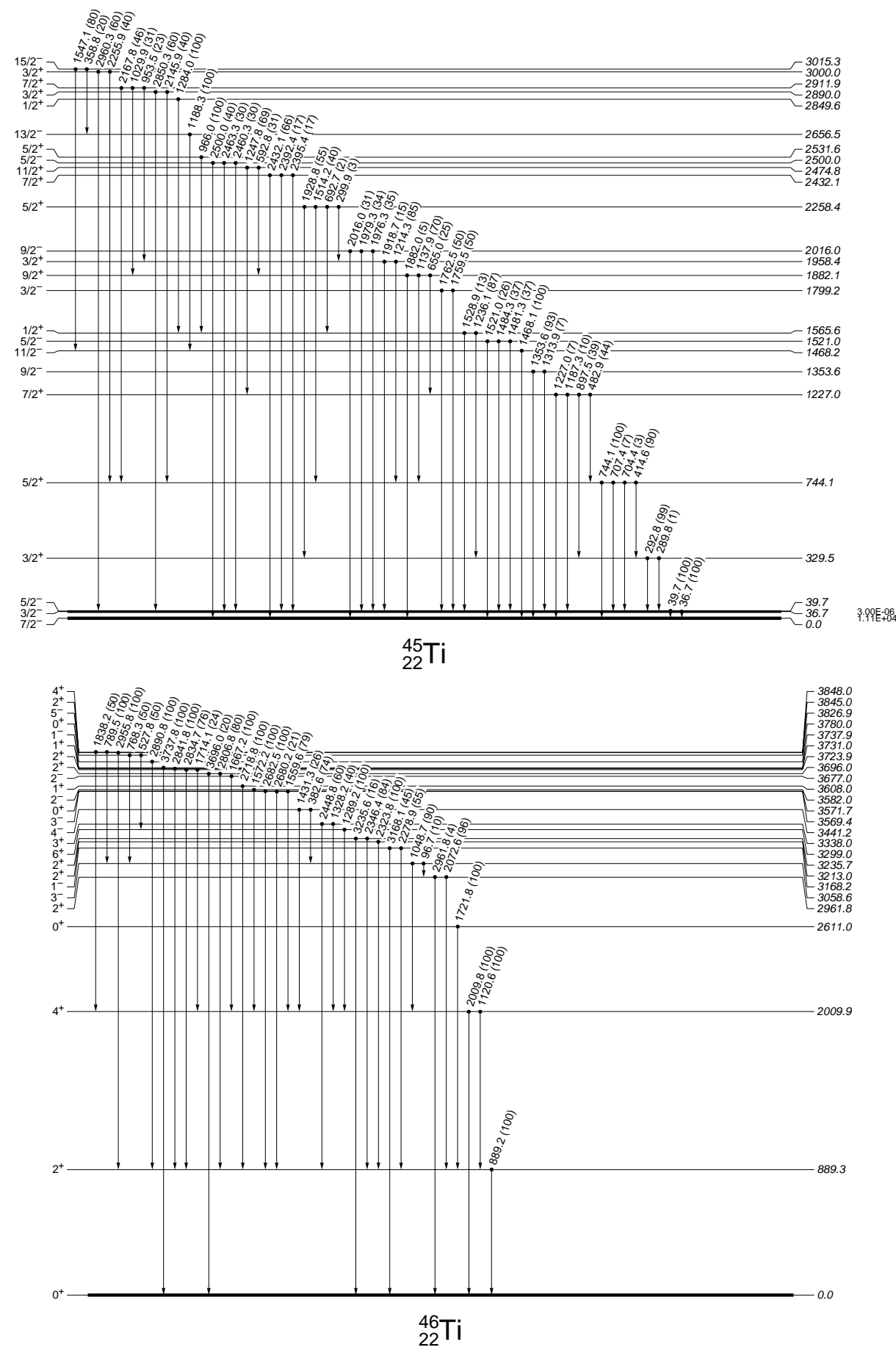

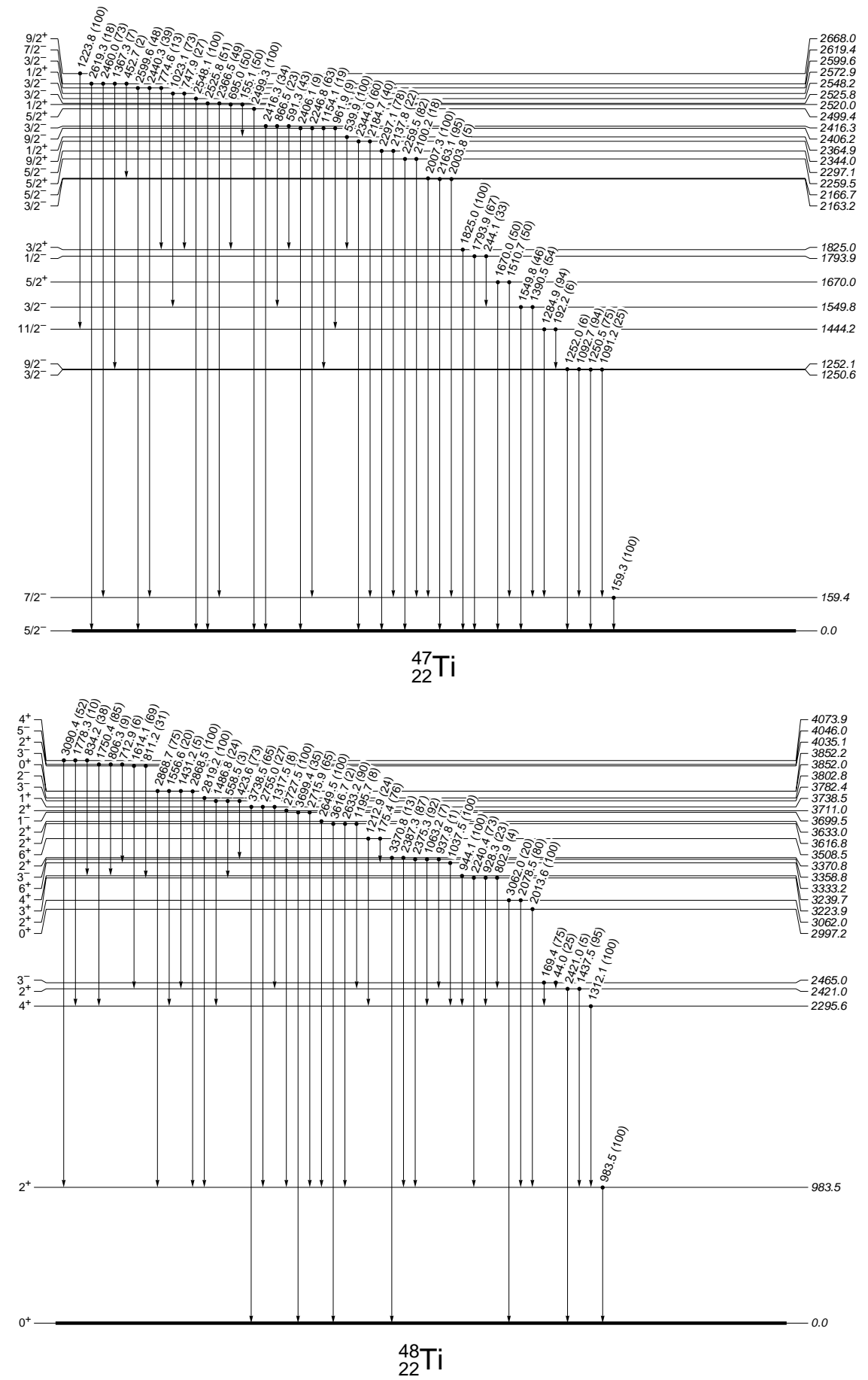

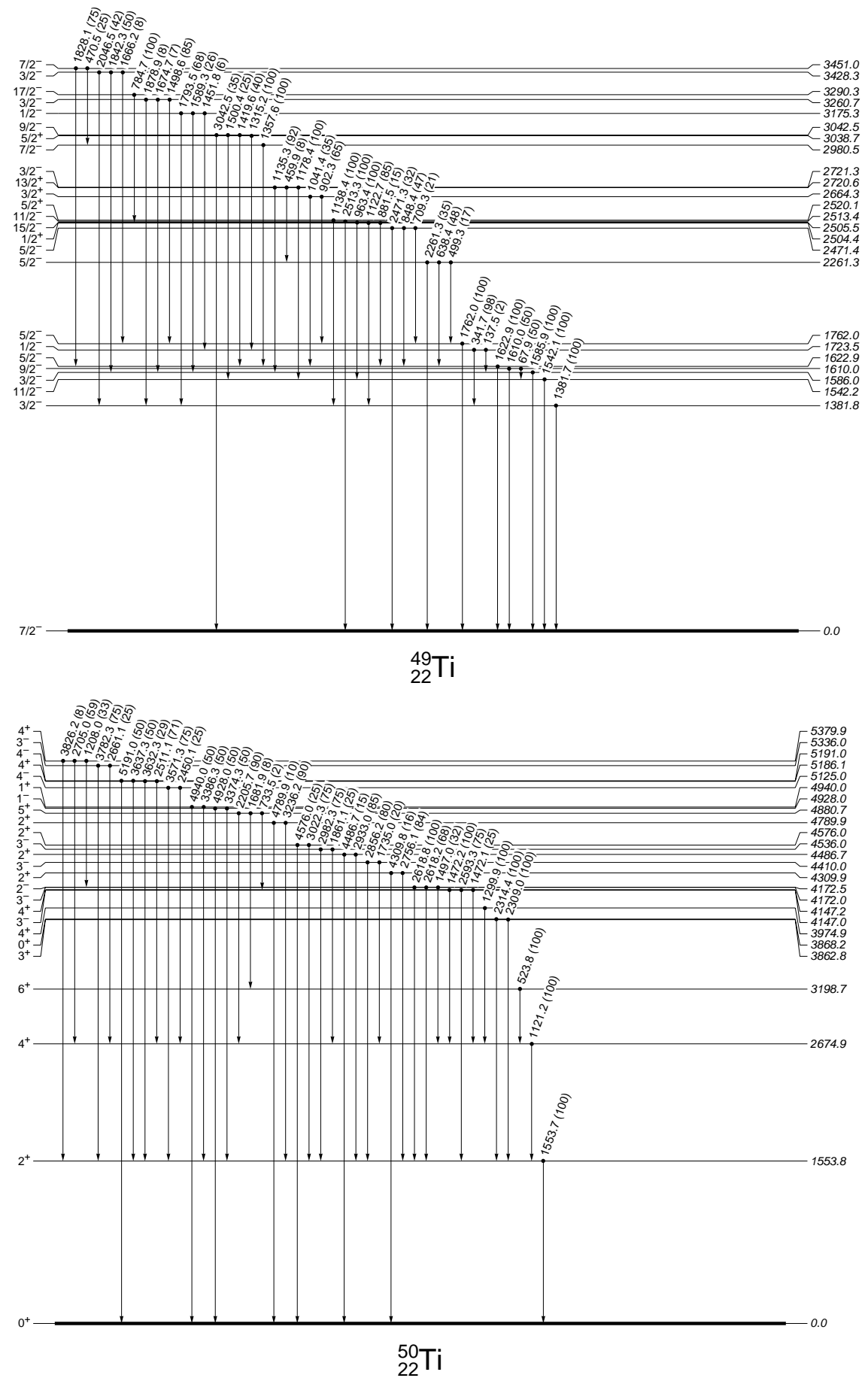

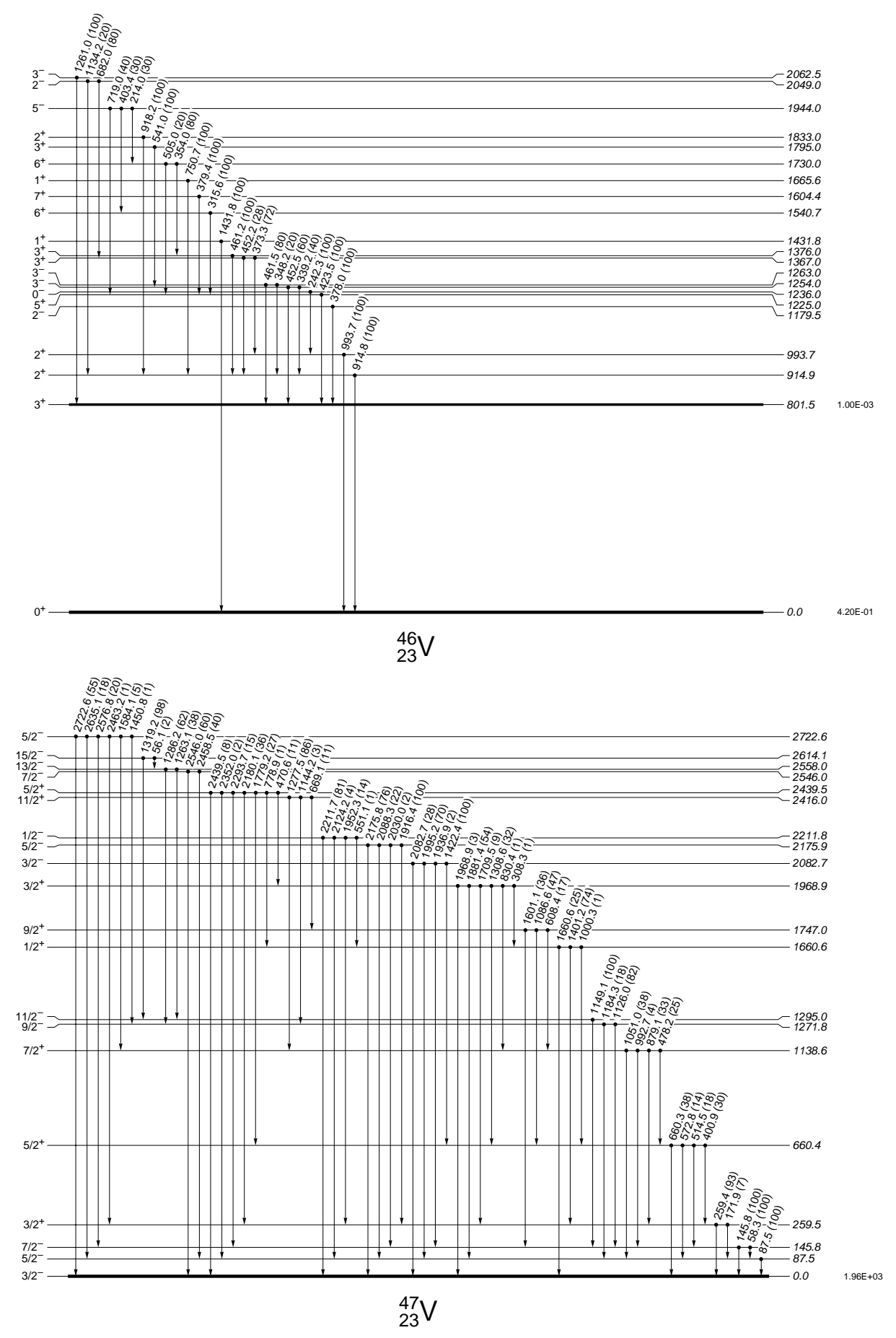

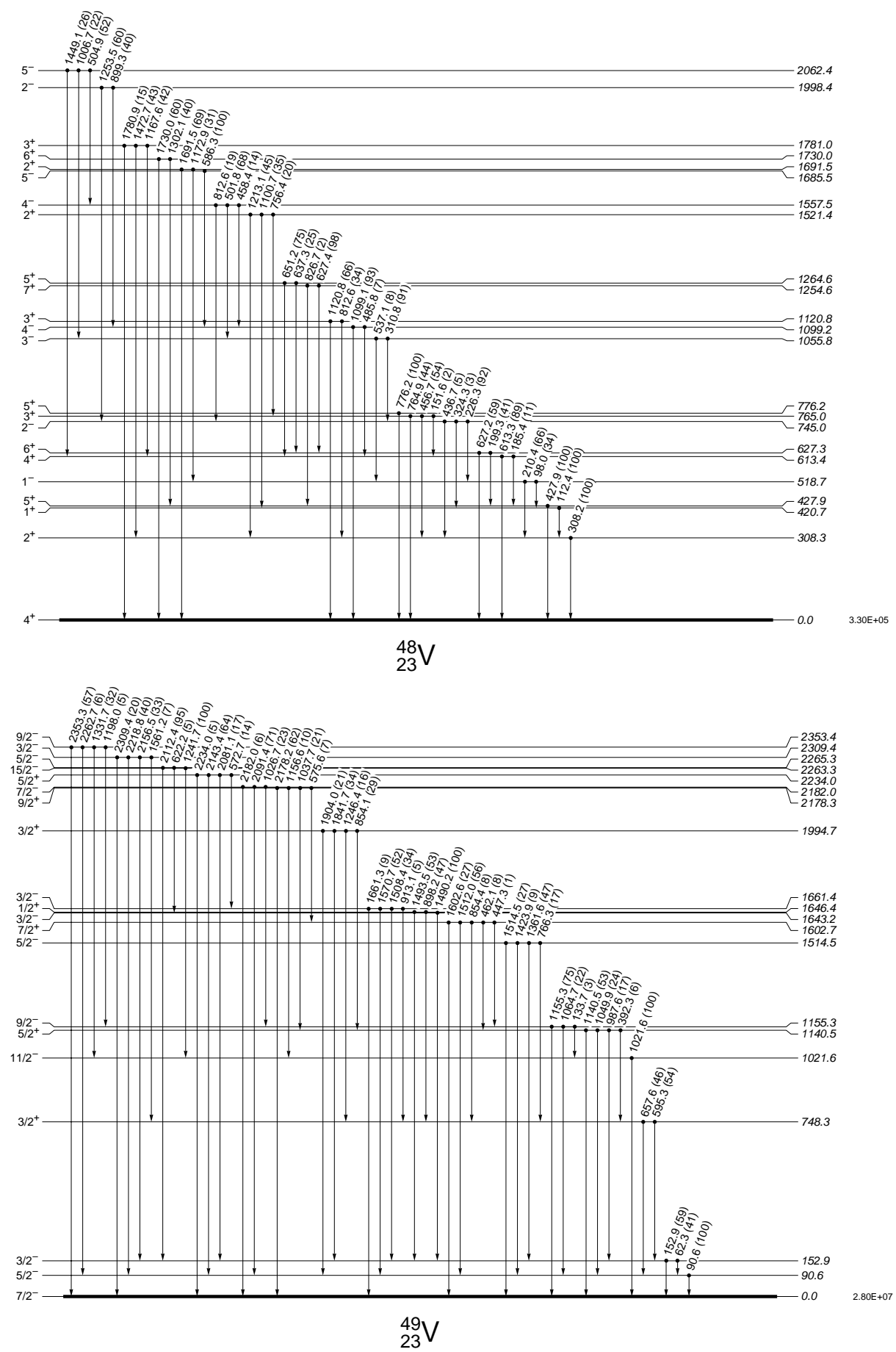

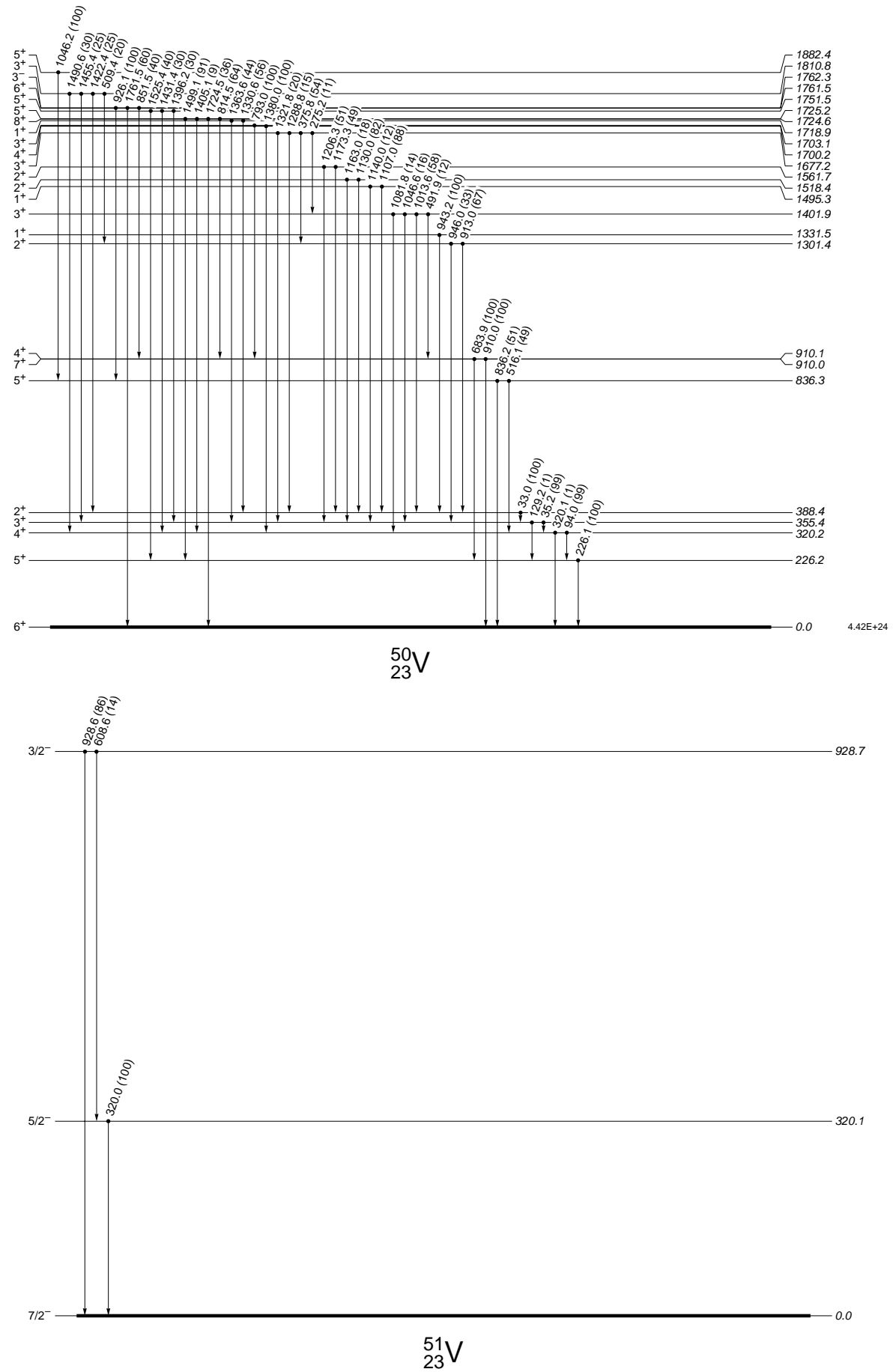

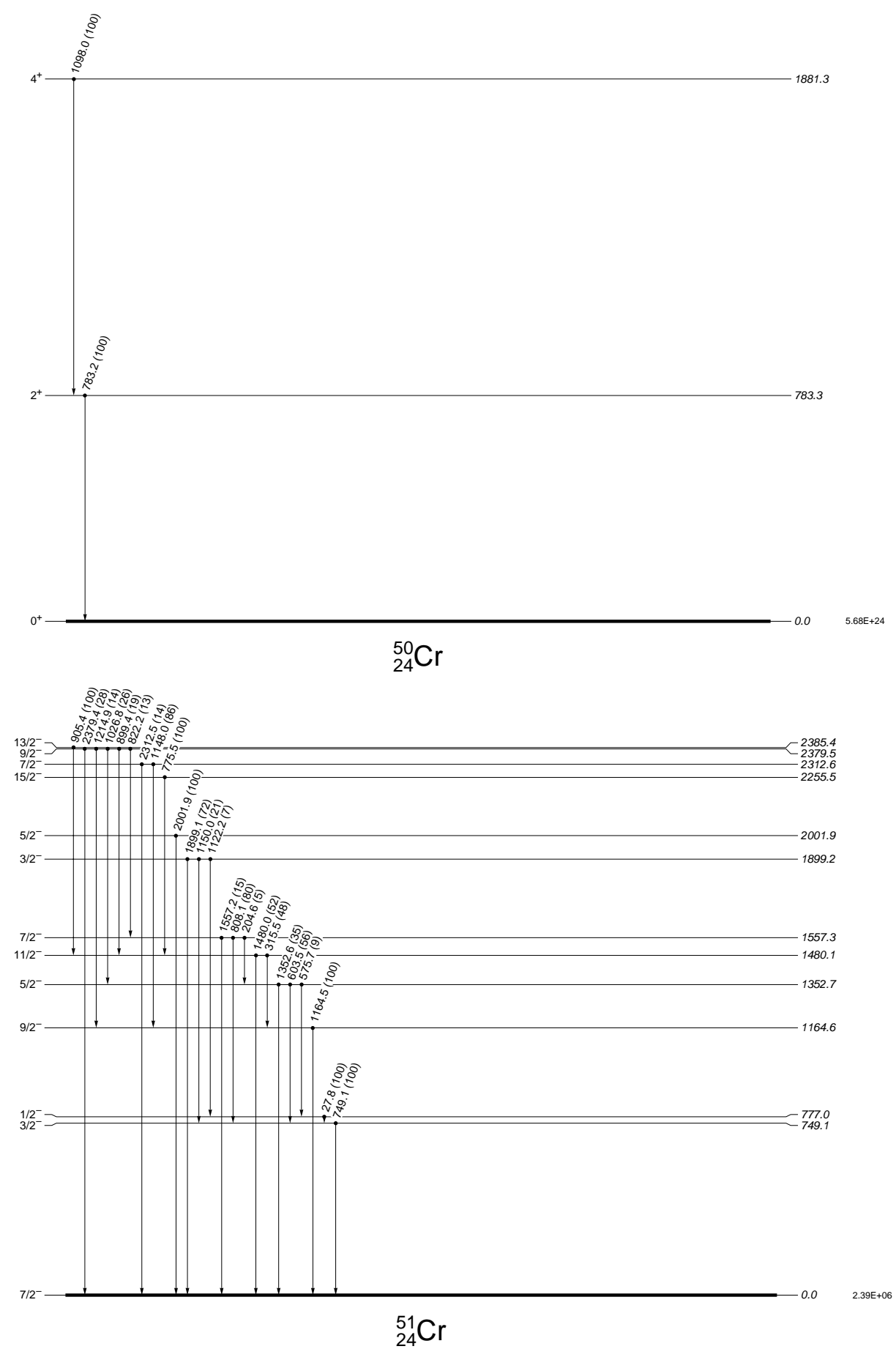

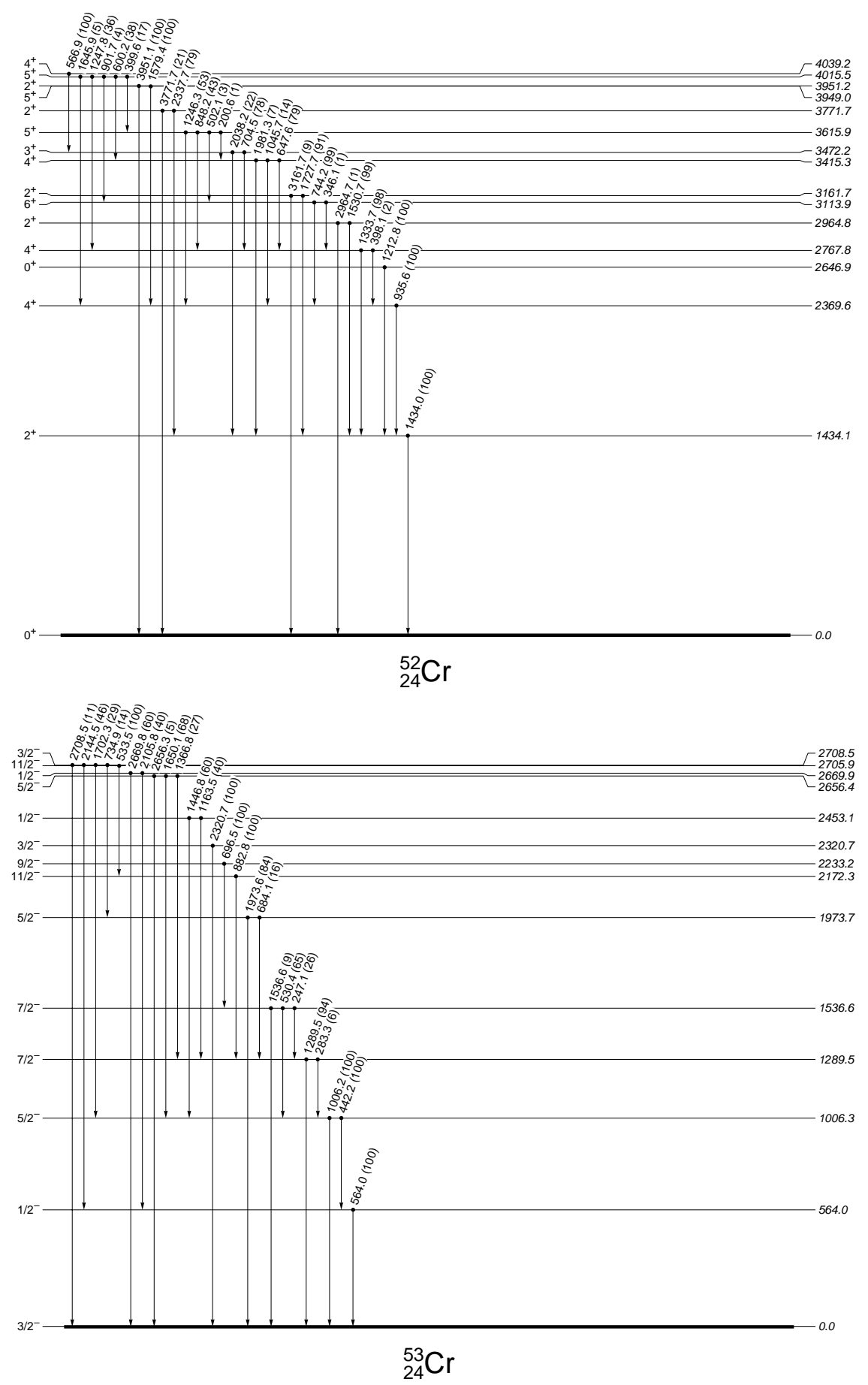

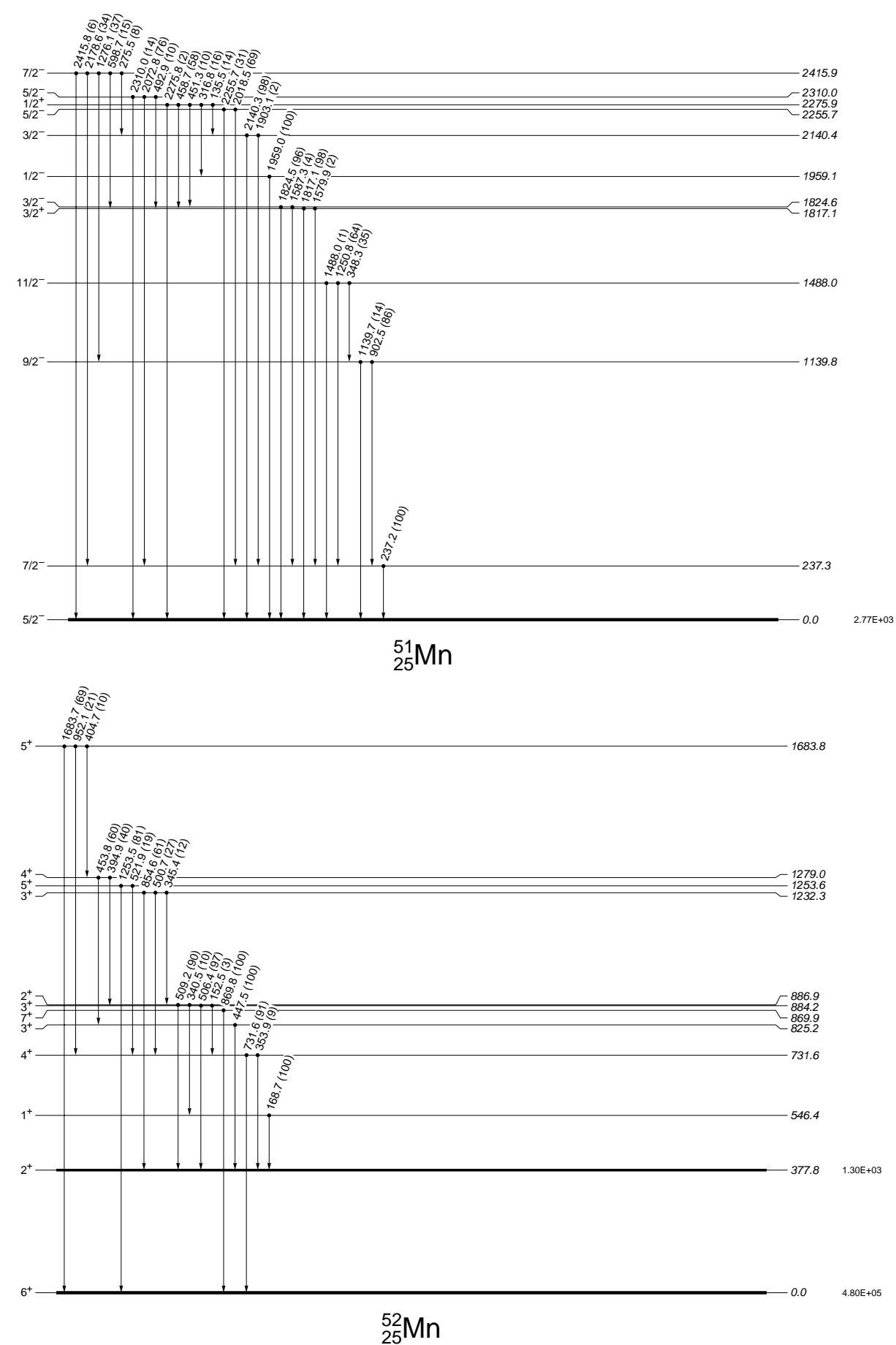

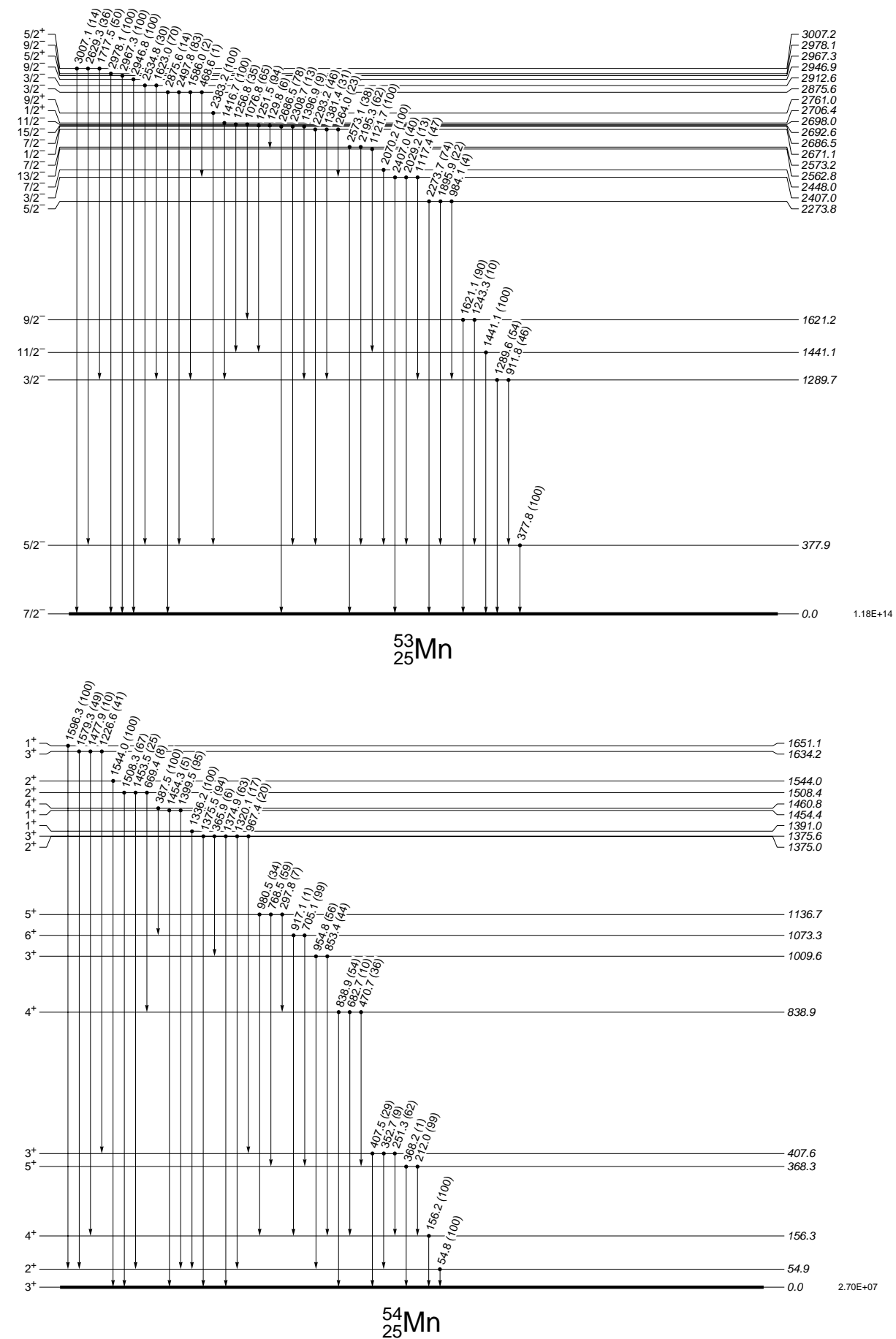

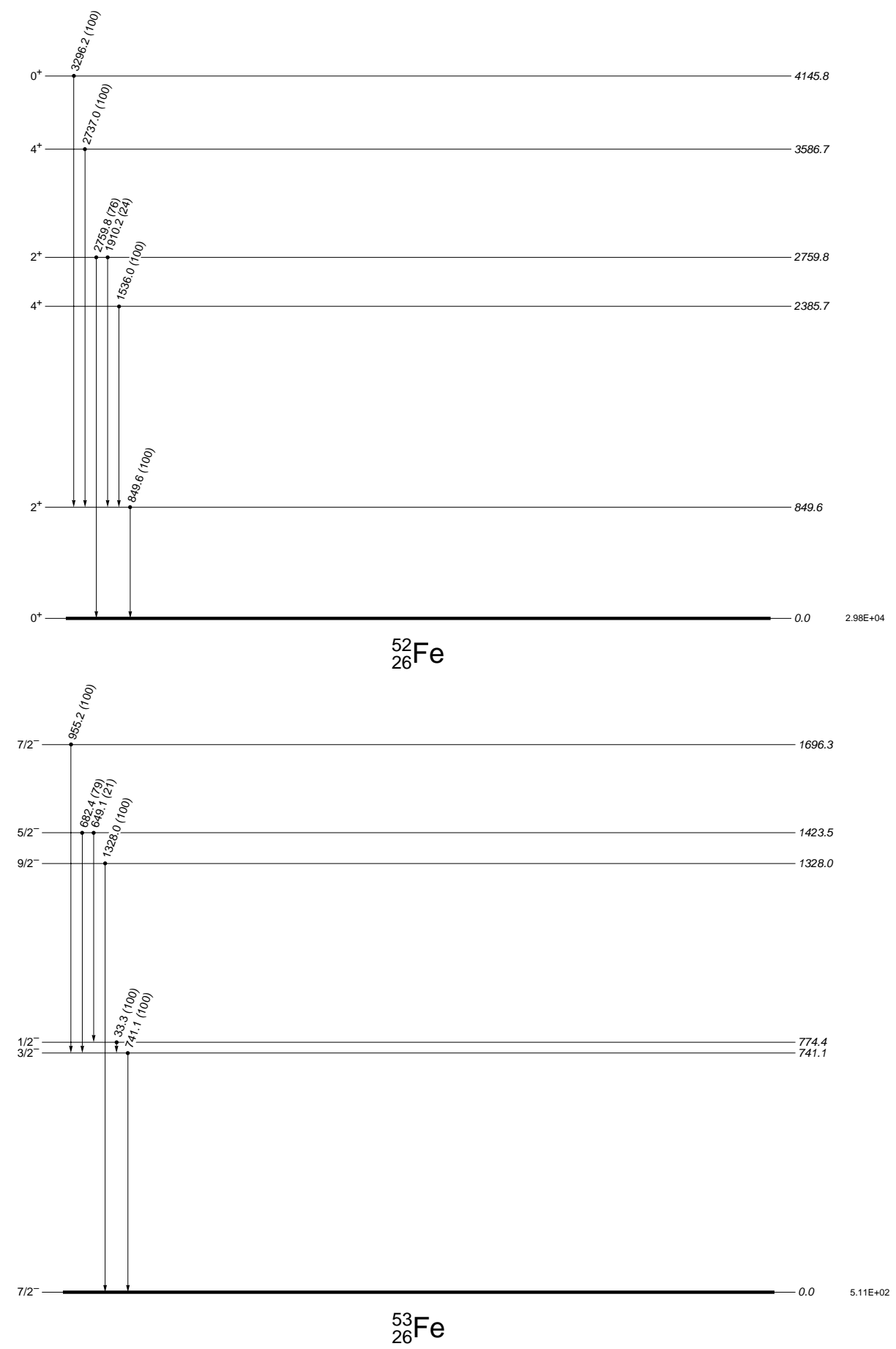


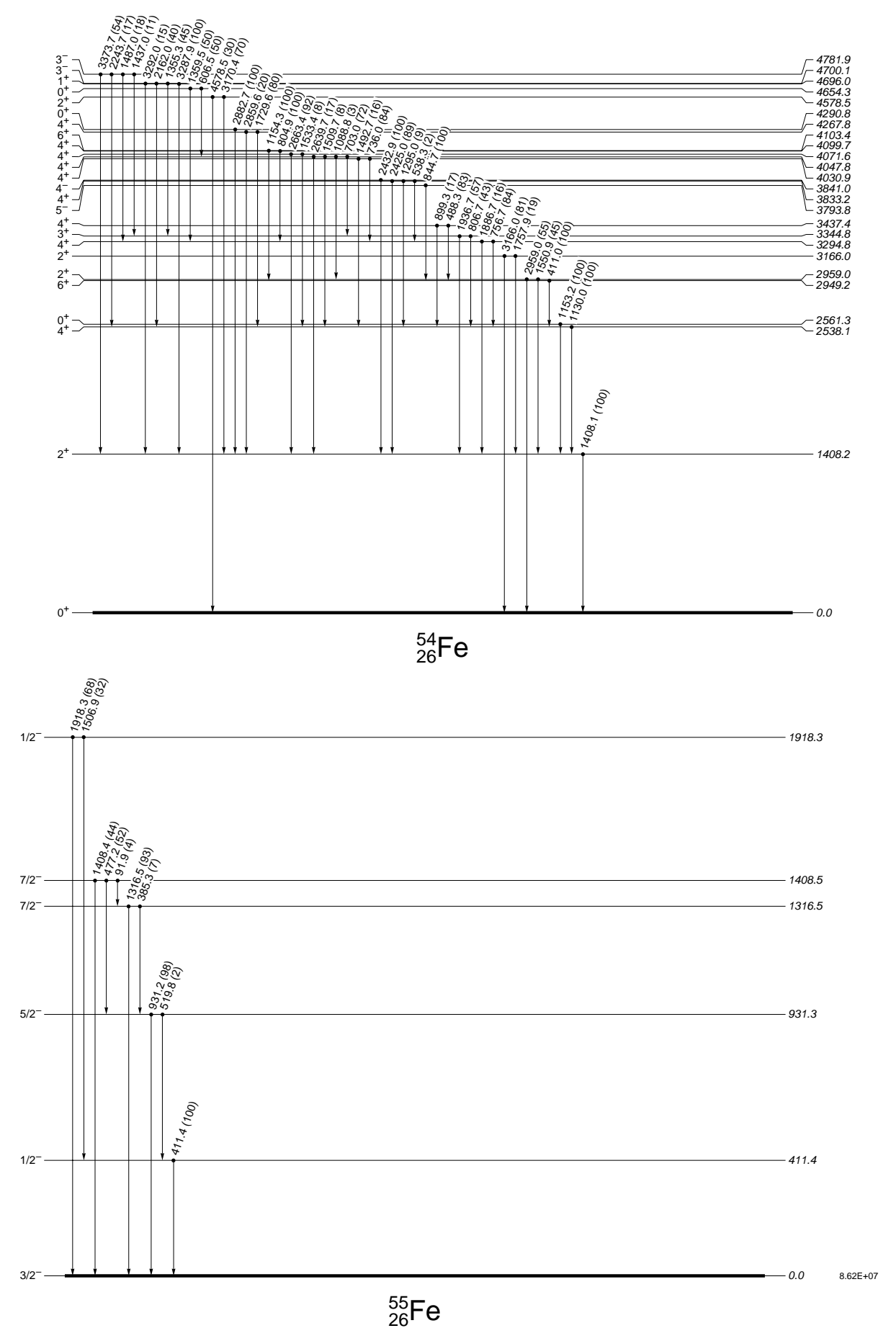




\section{A.5. Nuclear Level Density Parameters}

Table 9:: Adopted level density parameters

\begin{tabular}{|c|c|c|c|c|c|c|c|c|c|}
\hline${ }^{A} Z$ & $\begin{array}{c}\tilde{a}(A) \\
(\mathrm{MeV})^{-1}\end{array}$ & $\begin{array}{c}\Delta \\
(\mathrm{MeV})\end{array}$ & $\begin{array}{c}\delta W \\
(\mathrm{MeV})\end{array}$ & $\overline{\mathrm{x} / \mathrm{s}}$ & $\overline{\overline{\sigma^{2}}\left(E_{x}\right)}$ & $\begin{array}{c}E_{x} \\
(\mathrm{MeV})\end{array}$ & $\begin{array}{c}E_{0} \\
(\mathrm{MeV})\end{array}$ & $\begin{array}{c}T \\
(\mathrm{MeV})\end{array}$ & $\mathrm{N}$ \\
\hline${ }^{32} \mathrm{~S}$ & 4.090 & 3.273 & 4.460 & $\mathrm{~S}$ & 1.359 & 4.000 & -0.605 & 1.844 & 6 \\
\hline${ }^{33} \mathrm{~S}$ & 3.303 & 0.135 & 4.560 & $\mathrm{x}$ & 1.861 & 2.000 & -2.400 & 1.539 & 6 \\
\hline${ }^{34} \mathrm{~S}$ & 3.548 & 2.308 & 4.150 & $\mathrm{x}$ & 2.590 & 8.888 & -2.834 & 1.820 & 25 \\
\hline${ }^{35} \mathrm{~S}$ & 3.753 & 0.207 & 3.930 & $\mathrm{x}$ & 1.886 & 2.000 & -2.055 & 1.376 & 0 \\
\hline${ }^{36} \mathrm{~S}$ & 4.524 & 2.580 & 3.390 & $\mathrm{~s}$ & 1.885 & 4.500 & 0.604 & 1.162 & 2 \\
\hline${ }^{37} \mathrm{~S}$ & 4.632 & 0.155 & 3.650 & $\mathrm{~s}$ & 2.232 & 3.700 & -2.657 & 1.257 & 1 \\
\hline${ }^{38} \mathrm{~S}$ & 4.739 & 2.250 & 3.490 & $\mathrm{~s}$ & 1.538 & 3.000 & -0.438 & 1.411 & 1 \\
\hline${ }^{39} \mathrm{~S}$ & 4.847 & 0.417 & 3.260 & $\mathrm{~s}$ & 2.368 & 4.300 & -2.585 & 1.257 & 0 \\
\hline${ }^{40} \mathrm{~S}$ & 4.953 & 2.510 & 2.750 & $\mathrm{~s}$ & 2.602 & 7.700 & -1.384 & 1.368 & 0 \\
\hline${ }^{41} \mathrm{~S}$ & 5.060 & 0.377 & 2.580 & $\mathrm{~s}$ & 2.450 & 4.200 & -2.551 & 1.233 & U \\
\hline${ }^{42} \mathrm{~S}$ & 5.166 & 2.570 & 1.900 & $\mathrm{~S}$ & 2.553 & 6.700 & -0.537 & 1.262 & 0 \\
\hline${ }^{32} \mathrm{Cl}$ & 4.090 & -0.715 & 3.920 & $\mathrm{~s}$ & 1.360 & 0.000 & -5.040 & 1.993 & 3 \\
\hline${ }^{33} \mathrm{Cl}$ & 4.199 & 0.167 & 4.320 & $\mathrm{~s}$ & 1.749 & 2.000 & -1.857 & 1.207 & 1 \\
\hline${ }^{34} \mathrm{Cl}$ & 4.308 & -0.373 & 3.600 & $\mathrm{~s}$ & 2.297 & 4.500 & -4.130 & 1.454 & 4 \\
\hline${ }^{35} \mathrm{Cl}$ & 4.416 & 0.760 & 3.500 & $\mathrm{~S}$ & 1.658 & 2.000 & -1.249 & 1.221 & 2 \\
\hline${ }^{36} \mathrm{Cl}$ & 3.601 & -1.058 & 3.900 & $\mathrm{x}$ & .020 & 1.000 & -3.442 & 1.433 & 0 \\
\hline${ }^{37} \mathrm{Cl}$ & 4.632 & 0.190 & 3.380 & $\mathrm{~s}$ & .889 & 2.000 & -1.720 & 1.133 & 0 \\
\hline${ }^{38} \mathrm{Cl}$ & 4.903 & -0.815 & 3.460 & $\mathrm{x}$ & .904 & 1.000 & -2.645 & .070 & 2 \\
\hline${ }^{39} \mathrm{Cl}$ & 4.847 & 0.305 & $3.3^{\circ}$ & $\mathrm{s}$ & .556 & 5.574 & -3.684 & .378 & 6 \\
\hline${ }^{40} \mathrm{Cl}$ & 4.953 & -0.915 & 3 & $\mathrm{~s}$ & .706 & 5.335 & -5.659 & .434 & 0 \\
\hline${ }^{41} \mathrm{Cl}$ & 5.060 & 0.207 & 3. & $\mathrm{~s}$ & .749 & 6.366 & -4.429 & .418 & 0 \\
\hline${ }^{42} \mathrm{Cl}$ & 5.166 & .905 & 3 & $\mathrm{~s}$ & .782 & 5.166 & -5.467 & .394 & 0 \\
\hline${ }^{43} \mathrm{Cl}$ & 5.272 & -0.018 & 2.5 & $\mathrm{~s}$ & .827 & 5.971 & -4.474 & .382 & 0 \\
\hline${ }^{44} \mathrm{Cl}$ & 5.378 & 1.225 & 2.2 & $\mathrm{~s}$ & .792 & 4.100 & 5.168 & .318 & 0 \\
\hline${ }^{45} \mathrm{Cl}$ & 5.484 & 0.582 & 1.010 & $\mathrm{~s}$ & .905 & 6.200 & -3.489 & 1.358 & 0 \\
\hline${ }^{34} \mathrm{Ar}$ & 4.308 & 2.048 & 3.950 & $\mathrm{~s}$ & 1.817 & 4.000 & 0.013 & 1.196 & 1 \\
\hline${ }^{35} \mathrm{Ar}$ & 4.416 & 0.825 & 0 & $\mathrm{~s}$ & .914 & 3.000 & -1.282 & 1.208 & 2 \\
\hline${ }^{36} \mathrm{Ar}$ & 4.524 & 2.940 & 3.5 & $\mathrm{~s}$ & 2.116 & 6.000 & 0.412 & .239 & 9 \\
\hline${ }^{37} \mathrm{Ar}$ & 4.632 & 0.407 & 3.310 & $\mathrm{~s}$ & 831 & 2.000 & -1.460 & .134 & 0 \\
\hline${ }^{38} \mathrm{Ar}$ & 4.739 & 2.553 & 2.770 & $\mathrm{~s}$ & 1.942 & 4.400 & 0.638 & 1.134 & 11 \\
\hline${ }^{39} \mathrm{Ar}$ & 4.847 & 0.385 & 2.950 & $\mathrm{~s}$ & 2.480 & 4.983 & -3.103 & 1.331 & 19 \\
\hline${ }^{40} \mathrm{Ar}$ & 4.953 & 2.015 & 3.490 & $\mathrm{~S}$ & 2.713 & 8.304 & -2.750 & 1.441 & 50 \\
\hline${ }^{41} \mathrm{Ar}$ & 5.022 & -0.060 & 3.810 & $\mathrm{x}$ & .383 & 3.510 & -2.842 & 1.180 & 4 \\
\hline${ }^{42} \mathrm{Ar}$ & 5.166 & 2.023 & 3.480 & $\mathrm{~S}$ & 2.314 & 5.000 & -0.359 & 1.111 & 1 \\
\hline${ }^{43} \mathrm{Ar}$ & 5.272 & 0.003 & 3.180 & $\mathrm{~s}$ & 129 & 2.000 & -1.828 & 1.021 & 0 \\
\hline${ }^{44} \mathrm{Ar}$ & 5.378 & 2.045 & 2.420 & $\mathrm{~s}$ & 1.809 & 3.000 & 0.249 & 1.076 & 0 \\
\hline${ }^{45} \mathrm{Ar}$ & 5.484 & 0.553 & 1.570 & $\mathrm{~s}$ & 2.054 & 2.000 & -1.121 & 1.025 & 0 \\
\hline${ }^{46} \mathrm{Ar}$ & 5.589 & 2.037 & 1.150 & $\mathrm{~s}$ & 2.959 & 7.798 & -2.143 & 1.348 & 0 \\
\hline${ }^{47} \mathrm{Ar}$ & 5.694 & 0.412 & 1.010 & $\mathrm{~s}$ & 2.702 & 4.200 & -2.405 & 1.173 & 0 \\
\hline${ }^{48} \mathrm{Ar}$ & 5.799 & 2.138 & 1.350 & $\mathrm{~s}$ & 2.705 & 5.800 & -0.599 & 1.137 & 0 \\
\hline${ }^{35} \mathrm{~K}$ & 4.416 & 0.320 & 3.150 & $\mathrm{~s}$ & 1.796 & 2.000 & -1.652 & 1.198 & 0 \\
\hline${ }^{36} \mathrm{~K}$ & 4.524 & -0.895 & 3.340 & s & 2.091 & 2.000 & -3.329 & 1.232 & 0 \\
\hline${ }^{37} \mathrm{~K}$ & 4.632 & 0.375 & 3.160 & $\mathrm{~S}$ & 2.038 & 2.800 & -1.790 & 1.177 & 2 \\
\hline${ }^{38} \mathrm{~K}$ & 4.739 & -0.697 & 2.800 & $\mathrm{~S}$ & 2.636 & 5.500 & -5.350 & 1.496 & 2 \\
\hline${ }^{39} \mathrm{~K}$ & 4.847 & 0.627 & 2.370 & $\mathrm{~s}$ & 1.840 & 2.000 & -1.215 & 1.134 & 0 \\
\hline${ }^{40} \mathrm{~K}$ & 4.571 & -0.537 & 2.490 & $\mathrm{x}$ & 2.860 & 6.361 & -5.699 & 1.600 & 32 \\
\hline${ }^{41} \mathrm{~K}$ & 5.060 & 350 & 2.760 & $\mathrm{~s}$ & 2.900 & 7.844 & -5.291 & 1.535 & 40 \\
\hline${ }^{42} \mathrm{~K}$ & 4.517 & -0.810 & 3.220 & $\mathrm{x}$ & 3.352 & 10.300 & -9.474 & 1.915 & 25 \\
\hline
\end{tabular}


Table 9: (continued)

\begin{tabular}{|c|c|c|c|c|c|c|c|c|c|}
\hline$\overline{{ }^{A} Z}$ & $\begin{array}{c}\tilde{a}(A) \\
(\mathrm{MeV})^{-1}\end{array}$ & $\begin{array}{c}\Delta \\
(\mathrm{MeV})\end{array}$ & $\begin{array}{c}\delta W \\
(\mathrm{MeV})\end{array}$ & $\mathrm{x} / \mathrm{s}$ & $\overline{\overline{\sigma^{2}}\left(E_{x}\right)}$ & $\begin{array}{c}E_{x} \\
(\mathrm{MeV})\end{array}$ & $\begin{array}{c}E_{0} \\
(\mathrm{MeV})\end{array}$ & $\begin{array}{c}T \\
(\mathrm{MeV})\end{array}$ & $\overline{\mathrm{N}}$ \\
\hline${ }^{43} \mathrm{~K}$ & 5.272 & 0.202 & 2.910 & $\mathrm{~s}$ & 2.838 & 6.371 & -4.419 & 1.387 & 7 \\
\hline${ }^{44} \mathrm{~K}$ & 5.378 & -0.670 & 2.500 & $\mathrm{~s}$ & 2.859 & 5.239 & -5.063 & 1.358 & 0 \\
\hline${ }^{45} \mathrm{~K}$ & 5.484 & 0.303 & 1.760 & $\mathrm{~s}$ & 2.540 & 3.700 & -2.298 & 1.149 & 12 \\
\hline${ }^{46} \mathrm{~K}$ & 5.589 & -0.937 & 1.330 & $\mathrm{~s}$ & 2.246 & 1.000 & -2.734 & 1.030 & 3 \\
\hline${ }^{47} \mathrm{~K}$ & 5.694 & 0.415 & 0.460 & $\mathrm{~s}$ & 2.186 & 2.000 & -1.283 & 1.033 & 0 \\
\hline${ }^{48} \mathrm{~K}$ & 5.799 & -0.547 & 0.480 & $\mathrm{~s}$ & 2.944 & 4.400 & -4.109 & 1.269 & 0 \\
\hline${ }^{49} \mathrm{~K}$ & 5.904 & 0.107 & 1.070 & $\mathrm{~s}$ & 3.052 & 5.669 & -3.918 & 1.285 & 0 \\
\hline${ }^{50} \mathrm{~K}$ & 6.008 & -0.758 & 1.690 & $\mathrm{~s}$ & 2.800 & 3.100 & -3.618 & 1.115 & 0 \\
\hline${ }^{36} \mathrm{Ca}$ & 4.524 & 2.510 & 2.710 & $\mathrm{~s}$ & 2.599 & 9.177 & -2.498 & 1.586 & 0 \\
\hline${ }^{37} \mathrm{Ca}$ & 4.632 & 0.190 & 2.930 & $\mathrm{~s}$ & 2.626 & 6.744 & -4.747 & 1.545 & 0 \\
\hline${ }^{38} \mathrm{Ca}$ & 4.739 & 2.505 & 2.660 & $\mathrm{~s}$ & 1.844 & 4.000 & 0.646 & 1.139 & 3 \\
\hline${ }^{39} \mathrm{Ca}$ & 4.847 & 0.640 & 2.310 & $\mathrm{~s}$ & 1.837 & 2.000 & -1.209 & 1.138 & 0 \\
\hline${ }^{40} \mathrm{Ca}$ & 4.953 & 3.210 & 2.270 & $\mathrm{~s}$ & 1.629 & 4.000 & 0.681 & 1.364 & 7 \\
\hline${ }^{41} \mathrm{Ca}$ & 4.997 & 0.340 & 2.540 & $\mathrm{x}$ & 1.993 & 2.000 & -1.459 & 1.082 & 24 \\
\hline${ }^{42} \mathrm{Ca}$ & 5.166 & 2.478 & 2.520 & $\mathrm{~s}$ & 3.026 & 10.761 & -3.761 & 1.579 & 42 \\
\hline${ }^{43} \mathrm{Ca}$ & 5.430 & 0.115 & 3.000 & $\mathrm{x}$ & 2.701 & 5.356 & -3.807 & 1.282 & 16 \\
\hline${ }^{44} \mathrm{Ca}$ & 5.923 & 2.302 & 2.620 & $\mathrm{x}$ & 2.796 & 8.282 & -2.144 & 1.276 & 14 \\
\hline${ }^{45} \mathrm{Ca}$ & 5.775 & 0.153 & 2.340 & $\mathrm{x}$ & 2.138 & 2.000 & -1.546 & 0.960 & 3 \\
\hline${ }^{46} \mathrm{Ca}$ & 5.589 & 2.195 & 1.480 & $\mathrm{~s}$ & 2.739 & 6.495 & -0.982 & 1.219 & 6 \\
\hline${ }^{47} \mathrm{Ca}$ & 5.694 & 0.595 & 0.670 & $\mathrm{~s}$ & 2.116 & 2.000 & -1.068 & 1.026 & 3 \\
\hline${ }^{48} \mathrm{Ca}$ & 5.799 & 2.367 & 0.120 & $\mathrm{~s}$ & 2.240 & 4.000 & 0.668 & 1.030 & 5 \\
\hline${ }^{49} \mathrm{Ca}$ & 5.904 & 0.655 & 0.100 & $\mathrm{~s}$ & 2.161 & 2.000 & -0.983 & 1.016 & 0 \\
\hline${ }^{50} \mathrm{Ca}$ & 6.008 & 2.122 & 0.860 & $\mathrm{~s}$ & 1.950 & 3.000 & 0.369 & 1.044 & 1 \\
\hline${ }^{51} \mathrm{Ca}$ & 6.112 & 0.403 & 1.500 & $\mathrm{~s}$ & 2.276 & 2.000 & -1.169 & 0.926 & 0 \\
\hline${ }^{52} \mathrm{Ca}$ & 6.216 & 2.180 & 2.130 & $\mathrm{~s}$ & 1.936 & 3.000 & 0.594 & 0.947 & 1 \\
\hline${ }^{38} \mathrm{Sc}$ & 4.739 & -0.812 & 3.030 & $\mathrm{~S}$ & 2.656 & 5.635 & -5.672 & 1.510 & 0 \\
\hline${ }^{39} \mathrm{Sc}$ & 4.847 & 0.213 & 2.920 & $\mathrm{~s}$ & 2.691 & 6.559 & -4.557 & 1.482 & 0 \\
\hline${ }^{40} \mathrm{Sc}$ & 4.953 & -0.492 & 2.470 & $\mathrm{~s}$ & 2.169 & 2.000 & -2.638 & 1.141 & 0 \\
\hline${ }^{41} \mathrm{Sc}$ & 5.060 & 0.357 & 2.580 & $\mathrm{~s}$ & 1.981 & 2.000 & -1.417 & 1.067 & 1 \\
\hline${ }^{42} \mathrm{Sc}$ & 5.166 & -0.610 & 2.620 & $\mathrm{~s}$ & 1.568 & 0.000 & -4.012 & 1.602 & 5 \\
\hline${ }^{43} \mathrm{Sc}$ & 5.272 & 0.425 & 2.770 & $\mathrm{~s}$ & 3.127 & 9.401 & -6.412 & 1.603 & 38 \\
\hline${ }^{44} \mathrm{Sc}$ & 5.378 & -0.830 & 3.090 & $\mathrm{~s}$ & 3.058 & 7.037 & -6.809 & 1.495 & 27 \\
\hline${ }^{45} \mathrm{Sc}$ & 5.484 & 0.245 & 2.670 & $\mathrm{~s}$ & 3.293 & 10.068 & -7.281 & 1.624 & 35 \\
\hline${ }^{46} \mathrm{Sc}$ & 5.949 & -0.743 & 2.280 & $\mathrm{x}$ & 2.992 & 5.956 & -5.715 & 1.335 & 25 \\
\hline${ }^{47} \mathrm{Sc}$ & 5.694 & 0.425 & 1.320 & $\mathrm{~s}$ & 3.310 & 9.015 & -5.894 & 1.532 & 26 \\
\hline${ }^{48} \mathrm{Sc}$ & 5.799 & -0.967 & 0.950 & $\mathrm{~s}$ & 2.325 & 1.000 & -2.750 & 1.012 & 8 \\
\hline${ }^{49} \mathrm{Sc}$ & 5.904 & 0.320 & 0.210 & $\mathrm{~s}$ & 1.820 & 1.000 & -2.221 & 1.325 & 0 \\
\hline${ }^{50} \mathrm{Sc}$ & 6.008 & -0.702 & 0.330 & $\mathrm{~s}$ & 3.324 & 6.517 & -5.860 & 1.411 & 2 \\
\hline${ }^{51} \mathrm{Sc}$ & 6.112 & 0.210 & 0.860 & $\mathrm{~s}$ & 3.117 & 5.651 & -3.711 & 1.252 & 1 \\
\hline${ }^{52} \mathrm{Sc}$ & 6.216 & -0.728 & 1.670 & $\mathrm{~s}$ & 2.630 & 2.000 & -2.849 & 0.991 & 0 \\
\hline${ }^{53} \mathrm{Sc}$ & 6.320 & -0.158 & 2.600 & $\mathrm{~s}$ & 3.121 & 5.173 & -4.105 & 1.173 & 0 \\
\hline${ }^{54} \mathrm{Sc}$ & 6.424 & -1.120 & 3.270 & $\mathrm{~s}$ & 3.129 & 4.158 & -5.064 & 1.141 & 0 \\
\hline${ }^{39} \mathrm{Ti}$ & 4.847 & 0.313 & 2.830 & $\mathrm{~s}$ & 2.694 & 6.659 & -4.450 & 1.485 & 0 \\
\hline${ }^{40} \mathrm{Ti}$ & 4.953 & 2.090 & 3.270 & $\mathrm{~s}$ & 2.714 & 8.340 & -2.629 & 1.443 & 0 \\
\hline${ }^{41} \mathrm{Ti}$ & 5.060 & 0.245 & 2.890 & $\mathrm{~s}$ & 2.755 & 6.404 & -4.373 & 1.425 & 0 \\
\hline${ }^{42} \mathrm{Ti}$ & 5.166 & 2.378 & 2.770 & $\mathrm{~s}$ & 2.806 & 8.589 & -2.269 & 1.413 & 4 \\
\hline${ }^{43} \mathrm{Ti}$ & 5.272 & 0.375 & 2.960 & $\mathrm{~s}$ & 2.816 & 6.363 & -4.113 & 1.371 & 0 \\
\hline${ }^{44} \mathrm{Ti}$ & 5.378 & 2.973 & 3.040 & $\mathrm{~s}$ & 3.074 & 10.993 & -3.126 & 1.508 & 16 \\
\hline${ }^{45} \mathrm{Ti}$ & 5.484 & 0.202 & 3.430 & $\mathrm{~s}$ & 2.673 & 4.646 & -3.149 & 1.194 & 24 \\
\hline${ }^{46} \mathrm{Ti}$ & 5.589 & 2.160 & 3.020 & $\mathrm{~s}$ & 3.201 & 10.597 & -4.281 & 1.501 & 26 \\
\hline
\end{tabular}


Table 9: (continued)

\begin{tabular}{|c|c|c|c|c|c|c|c|c|c|}
\hline$\overline{{ }^{A} Z}$ & $\begin{array}{c}\tilde{a}(A) \\
(\mathrm{MeV})^{-1}\end{array}$ & $\begin{array}{c}\Delta \\
(\mathrm{MeV})\end{array}$ & $\begin{array}{c}\delta W \\
(\mathrm{MeV})\end{array}$ & $\bar{x} \mathrm{x} / \mathrm{s}$ & $\overline{\overline{\sigma^{2}}\left(E_{x}\right)}$ & $\begin{array}{c}E_{x} \\
(\mathrm{MeV})\end{array}$ & $\begin{array}{c}E_{0} \\
(\mathrm{MeV})\end{array}$ & $\begin{array}{c}T \\
(\mathrm{MeV})\end{array}$ & $\mathrm{N}$ \\
\hline${ }^{47} \mathrm{Ti}$ & 4.968 & -0.228 & 2.680 & $\mathrm{x}$ & 3.283 & 7.411 & -5.973 & 1.567 & 25 \\
\hline${ }^{48} \mathrm{Ti}$ & 5.867 & 2.073 & 1.690 & $\mathrm{x}$ & 3.247 & 9.827 & -3.655 & 1.438 & 25 \\
\hline${ }^{49} \mathrm{Ti}$ & 6.391 & 0.545 & 0.630 & $\mathrm{x}$ & 2.669 & 4.008 & -2.011 & 1.062 & 25 \\
\hline${ }^{50} \mathrm{Ti}$ & 6.026 & 2.250 & 0.240 & $\mathrm{x}$ & 2.835 & 6.069 & -0.545 & 1.151 & 24 \\
\hline${ }^{51} \mathrm{Ti}$ & 5.916 & 0.542 & 0.260 & $\mathrm{x}$ & 2.823 & 4.000 & -2.035 & 1.136 & 14 \\
\hline${ }^{52} \mathrm{Ti}$ & 6.216 & 2.083 & 1.030 & $\mathrm{~s}$ & 3.096 & 7.169 & -1.595 & 1.206 & 9 \\
\hline${ }^{53} \mathrm{Ti}$ & 6.320 & 0.350 & 1.730 & $\mathrm{~s}$ & 3.105 & 5.400 & -3.340 & 1.172 & 0 \\
\hline${ }^{54} \mathrm{Ti}$ & 6.424 & 2.077 & 2.370 & $\mathrm{~s}$ & 3.069 & 6.800 & -1.403 & 1.118 & 0 \\
\hline${ }^{55} \mathrm{Ti}$ & 6.527 & 0.475 & 2.820 & $\mathrm{~s}$ & 2.890 & 4.100 & -2.229 & 1.004 & 0 \\
\hline${ }^{56} \mathrm{Ti}$ & 6.630 & 2.313 & 3.110 & $\mathrm{~s}$ & 2.775 & 5.300 & 0.046 & 0.930 & 0 \\
\hline${ }^{42} \mathrm{~V}$ & 5.166 & -0.835 & 3.340 & $\mathrm{~s}$ & 2.775 & 5.236 & -5.416 & 1.387 & 0 \\
\hline${ }^{43} \mathrm{~V}$ & 5.272 & 0.135 & 3.230 & $\mathrm{~s}$ & 2.809 & 6.123 & -4.371 & 1.364 & 0 \\
\hline${ }^{44} \mathrm{~V}$ & 5.378 & -0.920 & 3.390 & $\mathrm{~s}$ & 2.835 & 4.989 & -5.374 & 1.335 & 1 \\
\hline${ }^{45} \mathrm{~V}$ & 5.484 & 0.297 & 3.460 & $\mathrm{~s}$ & 2.863 & 6.131 & -4.100 & 1.310 & 1 \\
\hline${ }^{46} \mathrm{~V}$ & 5.589 & -1.123 & 3.850 & $\mathrm{~s}$ & 1.908 & 0.000 & -2.630 & 0.926 & 8 \\
\hline${ }^{47} \mathrm{~V}$ & 5.694 & -0.048 & 3.360 & $\mathrm{~s}$ & 259 & 2.000 & -1.830 & 0.954 & 21 \\
\hline${ }^{48} \mathrm{~V}$ & 5.799 & -0.250 & 1.880 & $\mathrm{~s}$ & 3.188 & 6.935 & -5.558 & 1.403 & 22 \\
\hline${ }^{49} \mathrm{~V}$ & 5.904 & -0.293 & 1.990 & $\mathrm{~s}$ & 095 & 1.000 & -1.818 & 0.935 & 35 \\
\hline${ }^{50} \mathrm{~V}$ & 6.008 & -0.655 & 0.810 & $\mathrm{~s}$ & 340 & 6.846 & -6.081 & .419 & 24 \\
\hline${ }^{51} \mathrm{~V}$ & 645 & 0.020 & 0.5 & $\mathrm{x}$ & 599 & 4.617 & -3.316 & 1.263 & 30 \\
\hline${ }^{52} \mathrm{~V}$ & 346 & -0.715 & 0.5 & $\mathrm{x}$ & 874 & 3.064 & -3.475 & 1.097 & 24 \\
\hline${ }^{53} \mathrm{~V}$ & 320 & -0.140 & 1.360 & $\mathrm{~s}$ & 544 & 2.109 & -1.981 & 0.947 & 6 \\
\hline${ }^{54} \mathrm{~V}$ & 424 & -1.065 & 2.120 & $\mathrm{~s}$ & 164 & 13 & -4.944 & .168 & 0 \\
\hline${ }^{55} \mathrm{~V}$ & 527 & 0.005 & 2.5 & $\mathrm{~s}$ & 179 & 32 & -3.859 & .142 & 0 \\
\hline${ }^{56} \mathrm{~V}$ & 630 & 112 & 3.2 & $\mathrm{~s}$ & 35 & 66 & -4.978 & .110 & 0 \\
\hline${ }^{57} \mathrm{~V}$ & 6.733 & -0.093 & 3.490 & $\mathrm{~s}$ & 205 & 5.039 & -3.933 & 1.090 & 0 \\
\hline${ }^{44} \mathrm{Cr}$ & 378 & 2.238 & 2.9 & $\mathrm{~s}$ & 347 & 17 & -2.186 & 1.347 & 0 \\
\hline${ }^{45} \mathrm{Cr}$ & 5.484 & 0.118 & 3.110 & $\mathrm{~s}$ & 373 & 51 & -4.257 & 1.319 & 0 \\
\hline${ }^{46} \mathrm{Cr}$ & 5.589 & 60 & 3.220 & $\mathrm{~s}$ & 2.900 & 21 & -2.164 & 1.294 & 0 \\
\hline${ }^{47} \mathrm{Cr}$ & 5.694 & -0.133 & 3.5 & $\mathrm{~s}$ & 346 & 69 & -7.721 & .560 & 2 \\
\hline${ }^{48} \mathrm{Cr}$ & 5.799 & 2.042 & 3. & $\mathrm{~s}$ & 556 & 915 & -1.598 & 1.193 & 2 \\
\hline${ }^{49} \mathrm{Cr}$ & 5.904 & 0.930 & 1.400 & $\mathrm{~s}$ & 135 & 206 & -3.643 & 1.332 & 12 \\
\hline${ }^{50} \mathrm{Cr}$ & 6.008 & 1.348 & 1.760 & $\mathrm{~s}$ & 262 & .000 & -0.252 & 0.934 & 7 \\
\hline${ }^{51} \mathrm{Cr}$ & 6.203 & 0.527 & 0.360 & $\mathrm{x}$ & 199 & 520 & -3.748 & 1.294 & 12 \\
\hline${ }^{52} \mathrm{Cr}$ & 6.216 & 2.098 & 0.120 & $\mathrm{~s}$ & 306 & 456 & -2.416 & 1.325 & 15 \\
\hline${ }^{53} \mathrm{Cr}$ & 6.028 & 0.460 & 0.070 & $\mathrm{x}$ & 285 & 6.089 & -3.537 & 1.300 & 13 \\
\hline${ }^{54} \mathrm{Cr}$ & 6.277 & 2.195 & 0.740 & $\mathrm{x}$ & 350 & .322 & -2.210 & 1.284 & 9 \\
\hline${ }^{55} \mathrm{Cr}$ & 6.311 & 0.268 & 1.570 & $\mathrm{x}$ & 164 & 7.110 & -4.751 & 1.312 & 9 \\
\hline${ }^{56} \mathrm{Cr}$ & 6.630 & 2.192 & 1.980 & $\mathrm{~s}$ & 34 & 6.817 & -1.195 & 1.095 & 7 \\
\hline${ }^{57} \mathrm{Cr}$ & 6.733 & 0.255 & 2.670 & $\mathrm{~s}$ & 3.216 & 5.300 & -3.477 & 1.101 & 0 \\
\hline${ }^{58} \mathrm{Cr}$ & 6.836 & 2.148 & 2.900 & $\mathrm{~s}$ & 3.251 & 7.234 & -1.627 & 1.089 & 0 \\
\hline${ }^{45} \mathrm{Mn}$ & 5.484 & 168 & 0 & $\mathrm{~s}$ & 2.886 & .001 & -4.173 & 1.332 & 0 \\
\hline${ }^{46} \mathrm{Mn}$ & 5.589 & -0.770 & 2.700 & $\mathrm{~s}$ & 2.914 & 4.991 & -5.060 & 1.307 & 0 \\
\hline${ }^{47} \mathrm{Mn}$ & 5.694 & -0.060 & 2.830 & $\mathrm{~s}$ & 2.940 & 5.631 & -4.304 & 1.282 & 0 \\
\hline${ }^{48} \mathrm{Mn}$ & 5.799 & -0.335 & 2.150 & $\mathrm{~S}$ & 2.989 & 5.290 & -4.483 & 1.278 & 0 \\
\hline${ }^{49} \mathrm{Mn}$ & 5.904 & 1.110 & 1.330 & $\mathrm{~s}$ & 2.462 & 3.500 & -0.853 & 1.014 & 4 \\
\hline${ }^{50} \mathrm{Mn}$ & 6.008 & -0.960 & 1.170 & $\mathrm{~s}$ & 2.376 & 1.000 & -2.700 & 0.973 & 1 \\
\hline${ }^{51} \mathrm{Mn}$ & 6.112 & .815 & 0.370 & $\mathrm{~S}$ & 3.298 & 7.490 & -3.951 & 1.356 & 11 \\
\hline${ }^{52} \mathrm{Mn}$ & 6.216 & -0.815 & & $\mathrm{~S}$ & 2.912 & 3.000 & -3.590 & 1.132 & 11 \\
\hline${ }^{53} \mathrm{Mn}$ & 6.320 & 0.150 & -0.270 & $\mathrm{~s}$ & 3.128 & 4.929 & -3.239 & 1.204 & 21 \\
\hline
\end{tabular}


Table 9: (continued)

\begin{tabular}{|c|c|c|c|c|c|c|c|c|c|}
\hline$\overline{{ }^{A} Z}$ & $\begin{array}{c}\tilde{a}(A) \\
(\mathrm{MeV})^{-1}\end{array}$ & $\begin{array}{c}\Delta \\
(\mathrm{MeV})\end{array}$ & $\begin{array}{c}\delta W \\
(\mathrm{MeV})\end{array}$ & $\overline{x / s}$ & $\overline{\sigma^{2}\left(E_{x}\right)}$ & $\begin{array}{c}E_{x} \\
(\mathrm{MeV})\end{array}$ & $\begin{array}{c}E_{0} \\
(\mathrm{MeV})\end{array}$ & $\begin{array}{c}T \\
(\mathrm{MeV})\end{array}$ & $\overline{\mathrm{N}}$ \\
\hline${ }^{54} \mathrm{Mn}$ & 6.424 & -0.648 & -0.210 & $\mathrm{~s}$ & 3.469 & 6.278 & -5.533 & 1.345 & 17 \\
\hline${ }^{55} \mathrm{Mn}$ & 6.527 & -0.030 & 0.680 & $\mathrm{~s}$ & 2.857 & 3.137 & -2.392 & 1.020 & 12 \\
\hline${ }^{56} \mathrm{Mn}$ & 6.569 & -1.003 & 1.500 & $\mathrm{x}$ & 3.642 & 7.155 & -7.041 & 1.369 & 8 \\
\hline${ }^{57} \mathrm{Mn}$ & 6.733 & -0.043 & 1.960 & $\mathrm{~s}$ & 2.989 & 3.628 & -2.750 & 1.006 & 6 \\
\hline${ }^{58} \mathrm{Mn}$ & 6.836 & -0.955 & 2.560 & $\mathrm{~s}$ & 3.261 & 4.131 & -4.711 & 1.096 & 1 \\
\hline${ }^{59} \mathrm{Mn}$ & 6.939 & -0.035 & 2.800 & $\mathrm{~S}$ & 3.281 & 5.007 & -3.770 & 1.077 & 0 \\
\hline${ }^{48} \mathrm{Fe}$ & 5.799 & 2.038 & 2.060 & $\mathrm{~s}$ & 2.992 & 7.663 & -2.104 & 1.280 & 0 \\
\hline${ }^{49} \mathrm{Fe}$ & 5.904 & -0.488 & 2.430 & $\mathrm{~s}$ & 3.011 & 5.074 & -4.604 & 1.251 & 0 \\
\hline${ }^{50} \mathrm{Fe}$ & 6.008 & 1.310 & 1.950 & $\mathrm{~S}$ & 3.054 & 6.810 & -2.728 & 1.243 & 0 \\
\hline${ }^{51} \mathrm{Fe}$ & 6.112 & 0.627 & 0.640 & $\mathrm{~S}$ & 3.124 & 6.069 & -3.279 & 1.258 & 0 \\
\hline${ }^{52} \mathrm{Fe}$ & 6.216 & 1.940 & 0.530 & $\mathrm{~S}$ & 2.846 & 5.500 & -0.686 & 1.095 & 3 \\
\hline${ }^{53} \mathrm{Fe}$ & 6.320 & 0.647 & -0.730 & $\mathrm{~s}$ & 3.355 & 6.858 & -3.678 & 1.322 & 5 \\
\hline${ }^{54} \mathrm{Fe}$ & 6.424 & 1.983 & -1.010 & $\mathrm{~s}$ & 3.295 & 7.426 & -1.797 & 1.261 & 25 \\
\hline${ }^{55} \mathrm{Fe}$ & 6.409 & 0.490 & -0.970 & $\mathrm{x}$ & 3.236 & 5.246 & -2.840 & 1.210 & 5 \\
\hline${ }^{56} \mathrm{Fe}$ & 6.630 & 1.812 & 0.050 & $\mathrm{~s}$ & 3.462 & 8.163 & -2.691 & 1.272 & 33 \\
\hline${ }^{57} \mathrm{Fe}$ & 6.498 & 0.217 & 0.720 & $\mathrm{x}$ & 3.554 & 6.917 & -4.608 & 1.297 & 31 \\
\hline${ }^{58} \mathrm{Fe}$ & 6.690 & 2.082 & 1.220 & $\mathrm{x}$ & 3.660 & 9.547 & -3.383 & 1.314 & 32 \\
\hline${ }^{59} \mathrm{Fe}$ & 6.857 & 0.100 & 1.940 & $\mathrm{x}$ & 3.041 & 3.665 & -2.530 & 0.985 & 28 \\
\hline${ }^{60} \mathrm{Fe}$ & 7.041 & 2.085 & 2.070 & $\mathrm{~s}$ & 3.447 & 7.806 & -2.125 & 1.133 & 24 \\
\hline${ }^{50} \mathrm{Co}$ & 6.008 & -0.627 & 0.980 & $\mathrm{~s}$ & 3.084 & 4.873 & -4.601 & 1.268 & 0 \\
\hline${ }^{51} \mathrm{Co}$ & 6.112 & 0.690 & 0.350 & $\mathrm{~s}$ & 3.134 & 6.131 & -3.196 & 1.266 & 0 \\
\hline${ }^{52} \mathrm{Co}$ & 6.216 & -0.840 & 0.120 & $\mathrm{~s}$ & 3.171 & 4.545 & -4.668 & 1.253 & 0 \\
\hline${ }^{53} \mathrm{Co}$ & 6.320 & 0.770 & -0.860 & $\mathrm{~s}$ & 3.235 & 6.100 & -2.948 & 1.262 & 1 \\
\hline${ }^{54} \mathrm{Co}$ & 6.424 & -1.045 & -1.170 & $\mathrm{~s}$ & 3.388 & 5.000 & -5.214 & 1.309 & 1 \\
\hline${ }^{55} \mathrm{Co}$ & 6.527 & 0.857 & -2.180 & $\mathrm{~s}$ & 3.298 & 5.798 & -2.508 & 1.245 & 9 \\
\hline${ }^{56} \mathrm{Co}$ & 6.630 & -0.870 & -1.540 & $\mathrm{~s}$ & 2.680 & 1.244 & -2.651 & 0.996 & 7 \\
\hline${ }^{57} \mathrm{Co}$ & 6.733 & 0.295 & -0.890 & $\mathrm{~s}$ & 3.313 & 5.190 & -3.124 & 1.178 & 7 \\
\hline${ }^{58} \mathrm{Co}$ & 6.836 & -0.870 & 0.110 & $\mathrm{~s}$ & 3.497 & 5.213 & -5.188 & 1.227 & 11 \\
\hline${ }^{59} \mathrm{Co}$ & 6.939 & -0.007 & 0.770 & $\mathrm{~s}$ & 3.031 & 3.380 & -2.490 & 0.991 & 31 \\
\hline${ }^{60} \mathrm{Co}$ & 7.131 & -0.870 & 1.390 & $\mathrm{x}$ & 3.351 & 4.171 & -4.526 & 1.088 & 49 \\
\hline
\end{tabular}


B. Modeled Cross Sections vs. Experiment

B.1. Modeled vs. Measured Cross Sections - (n, $\gamma)$
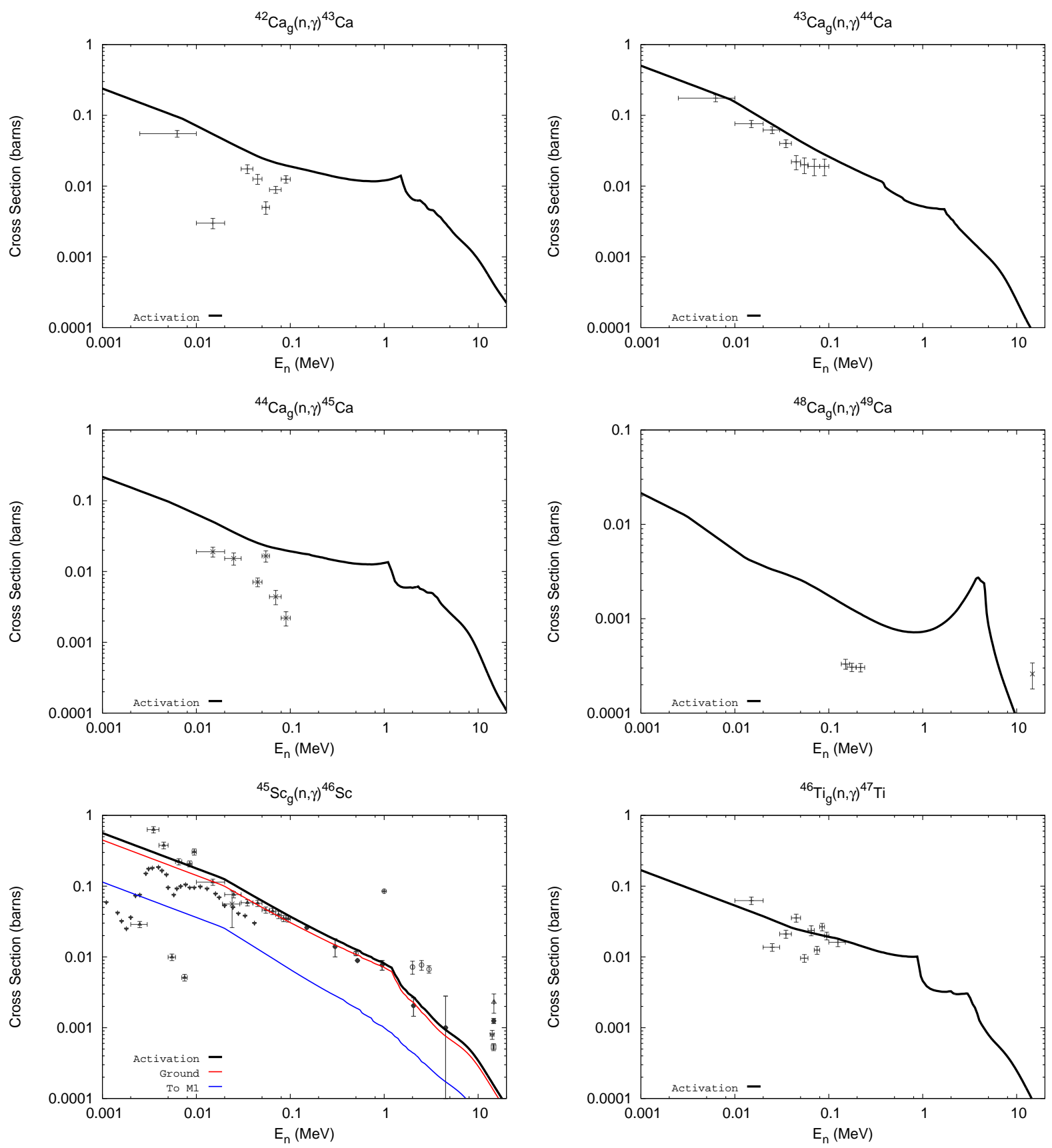

Fig. 22.- Measured vs. calculated cross sections for $(\mathrm{n}, \gamma)$ reactions 

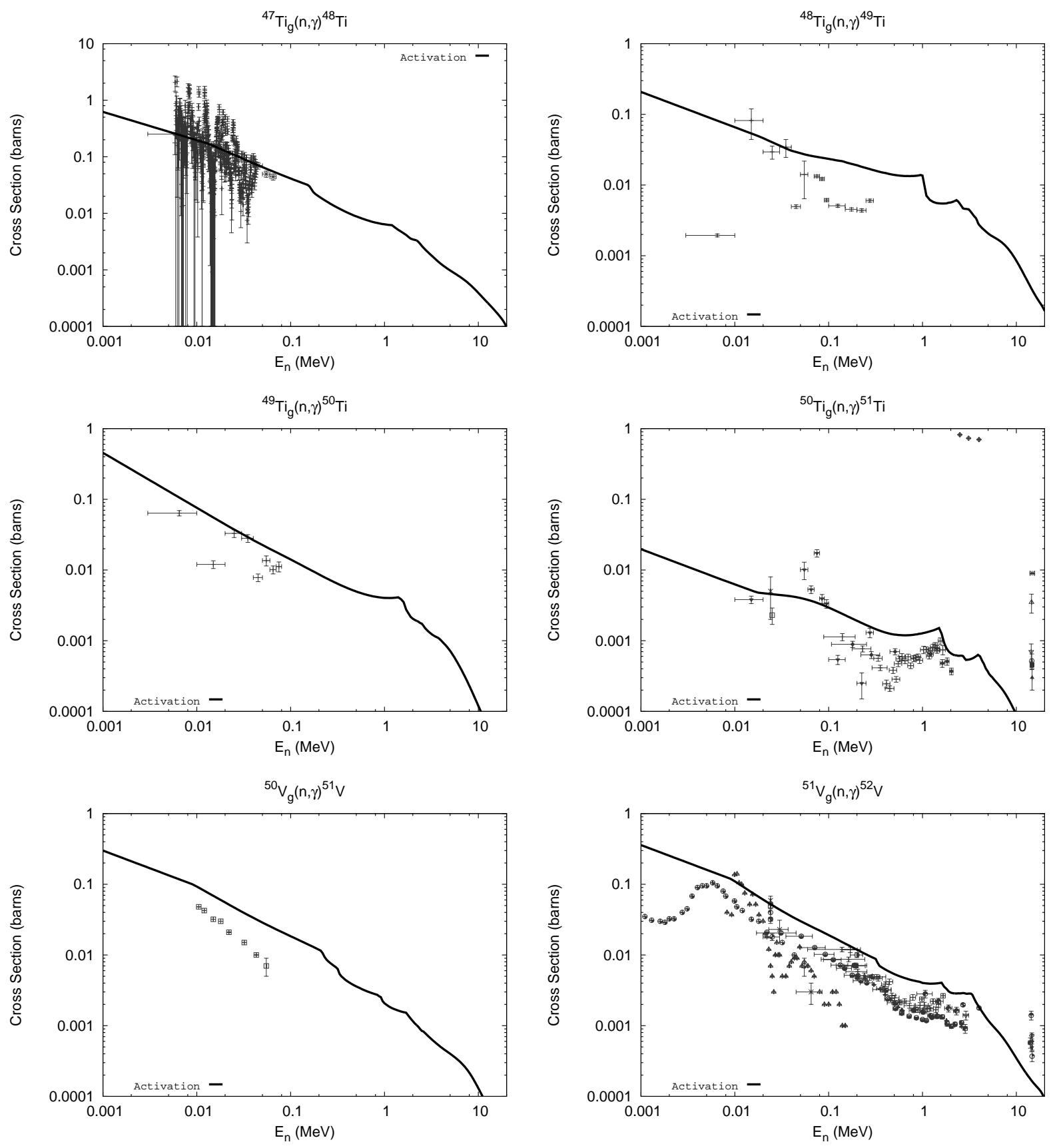

Fig. 22.- (continued) 

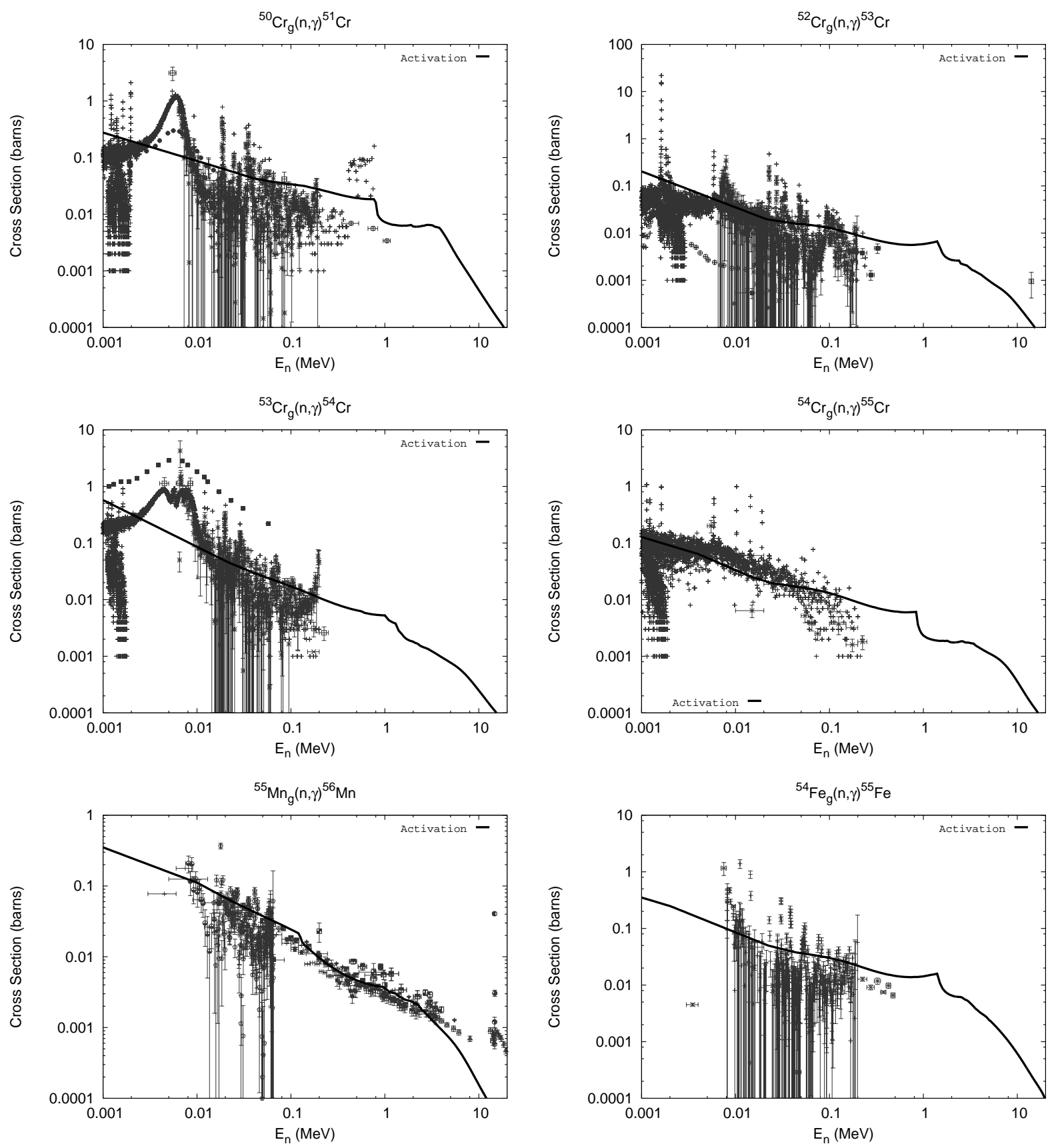

Fig. 22.- (continued) 

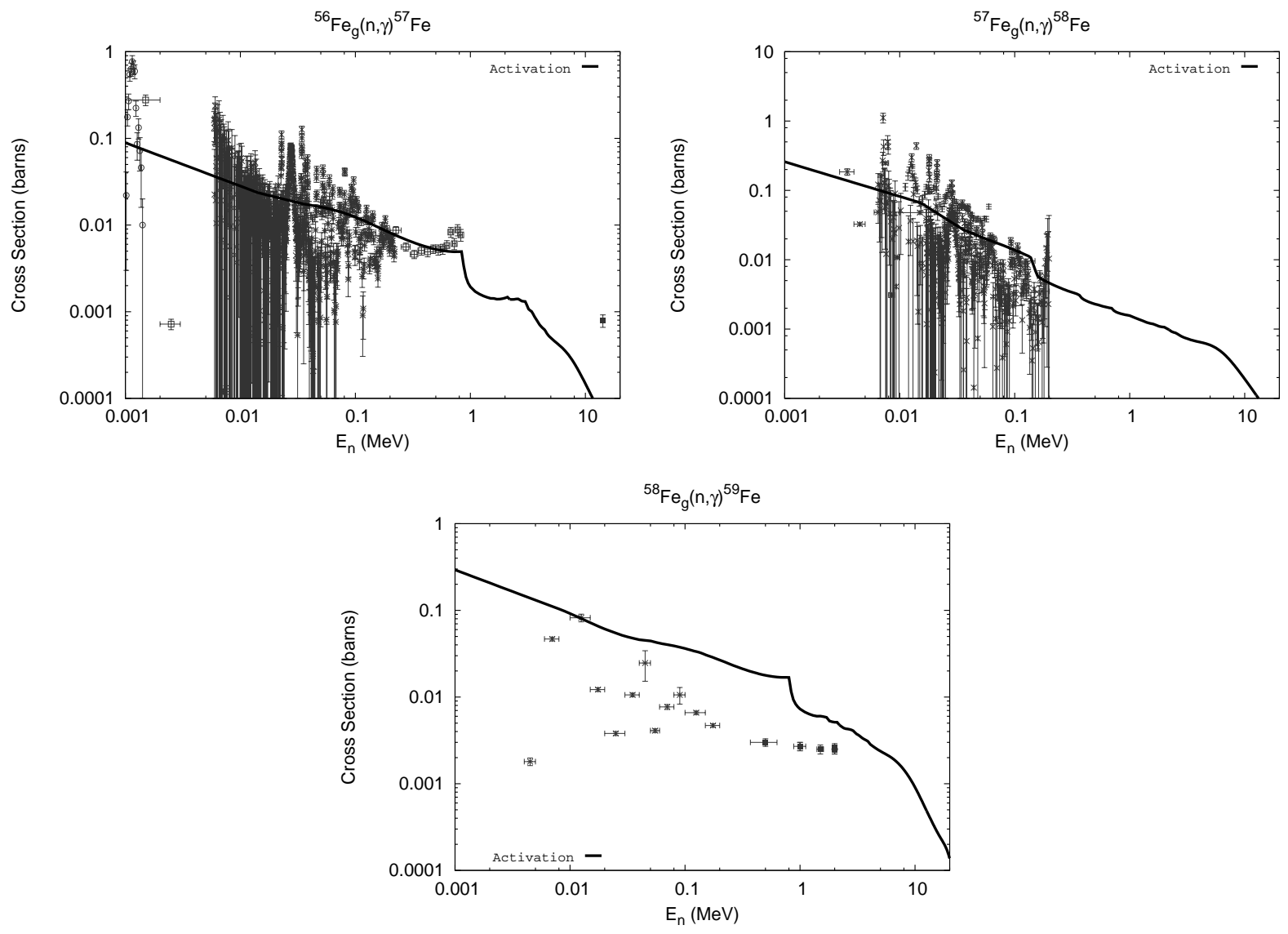

Fig. 22.- (continued) 


\section{B.2. Maxwellian averaged $(n, \gamma)$ capture cross sections}
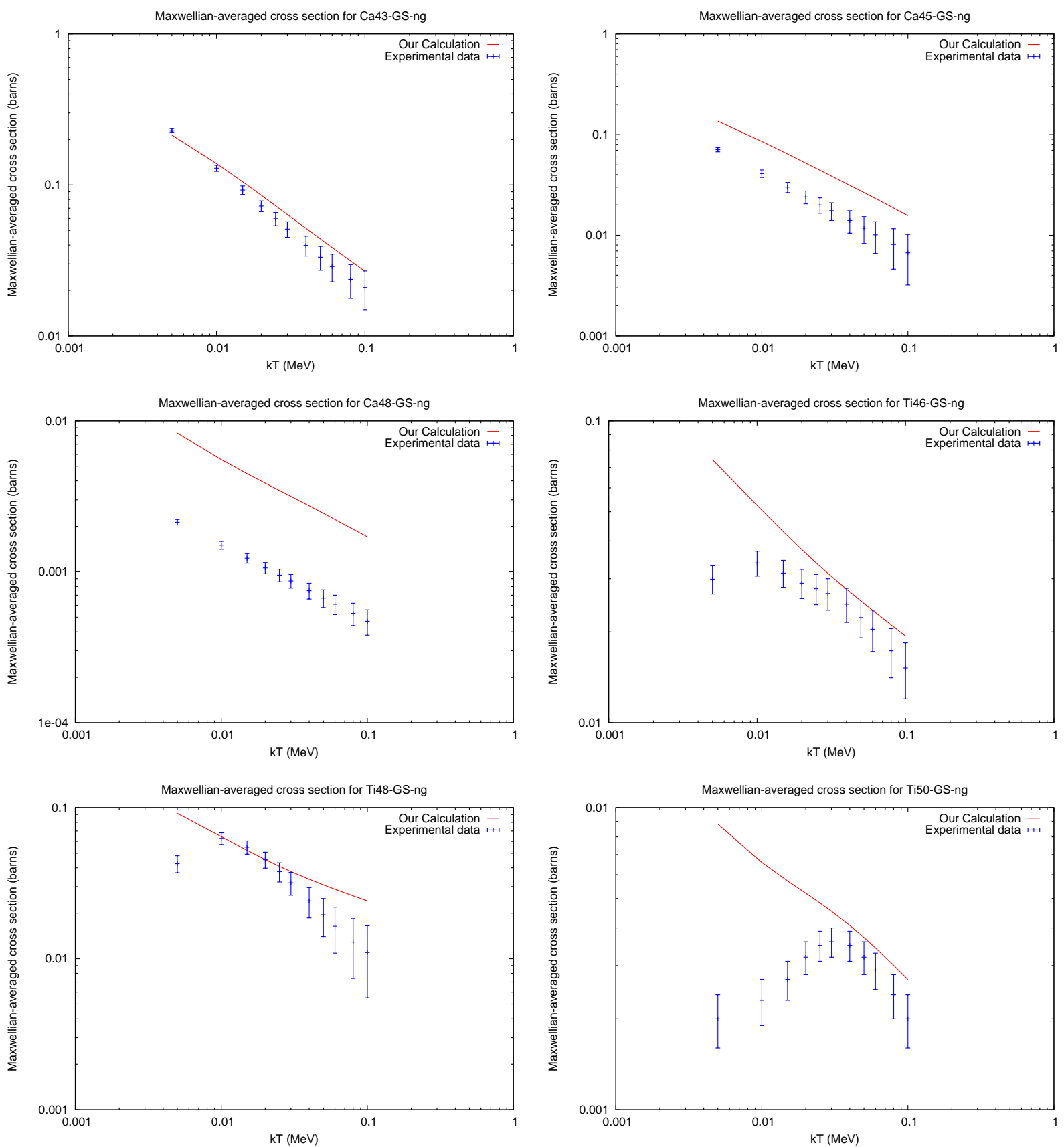

Fig. 23.- Measured vs. calculated maxwellian averaged $(\mathrm{n}, \gamma)$ cross sections 

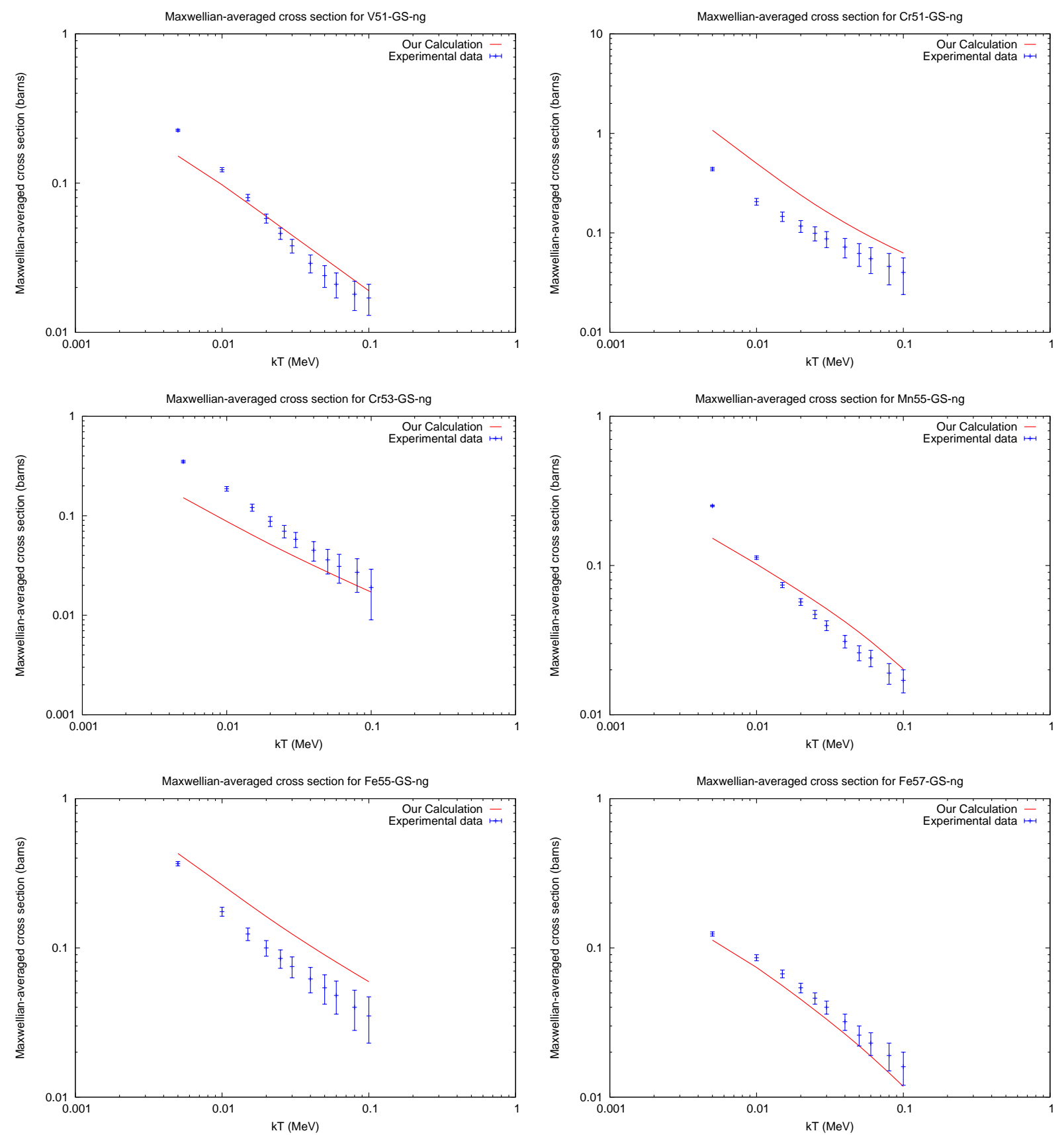

Fig. 23.- (continued) 


\section{B.3. Modeled vs. Measured Cross Sections - $(\mathbf{n}, 2 \mathrm{n})$}
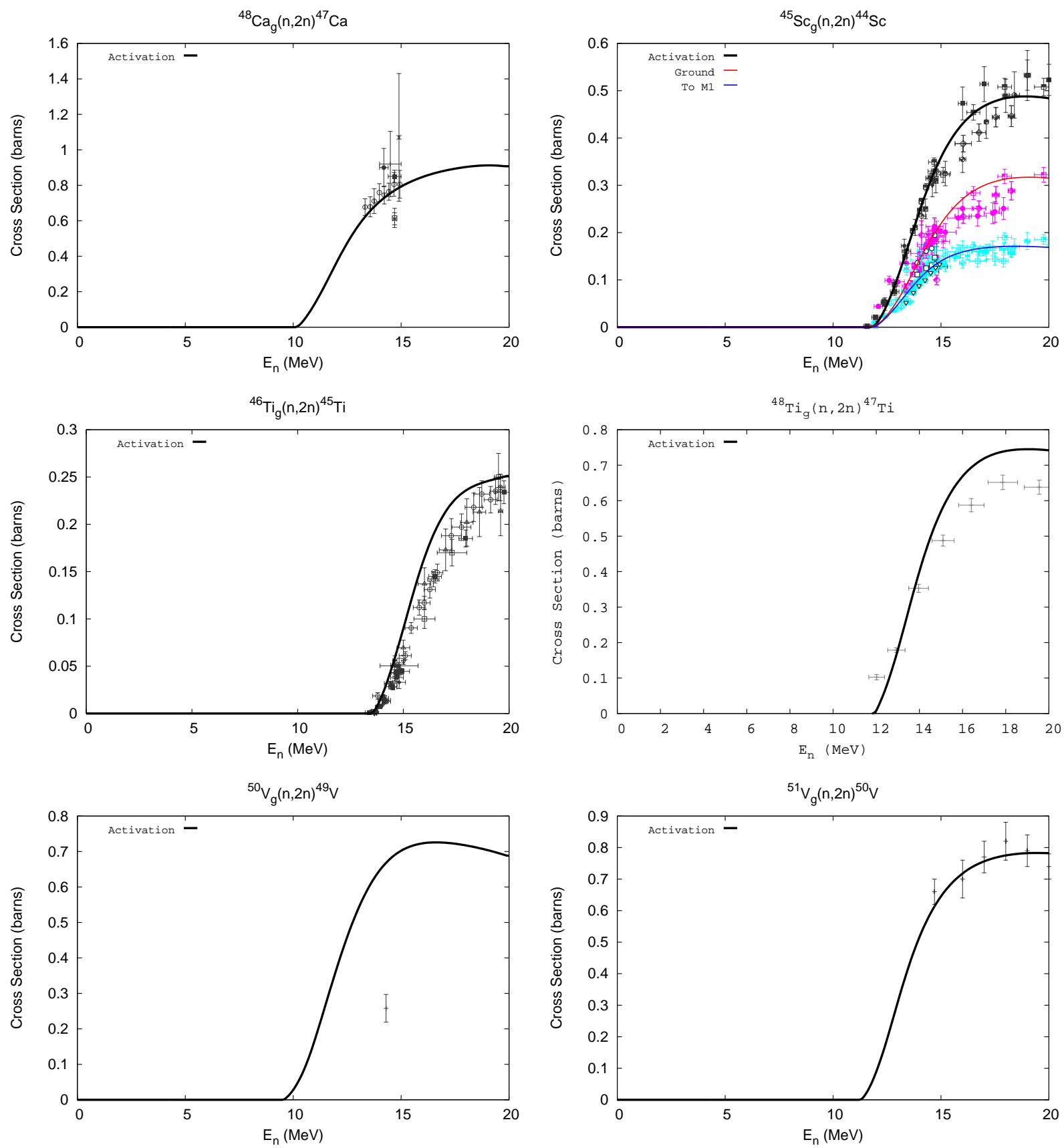

Fig. 24.- Measured vs. calculated cross sections for $(\mathrm{n}, 2 \mathrm{n})$ reactions 

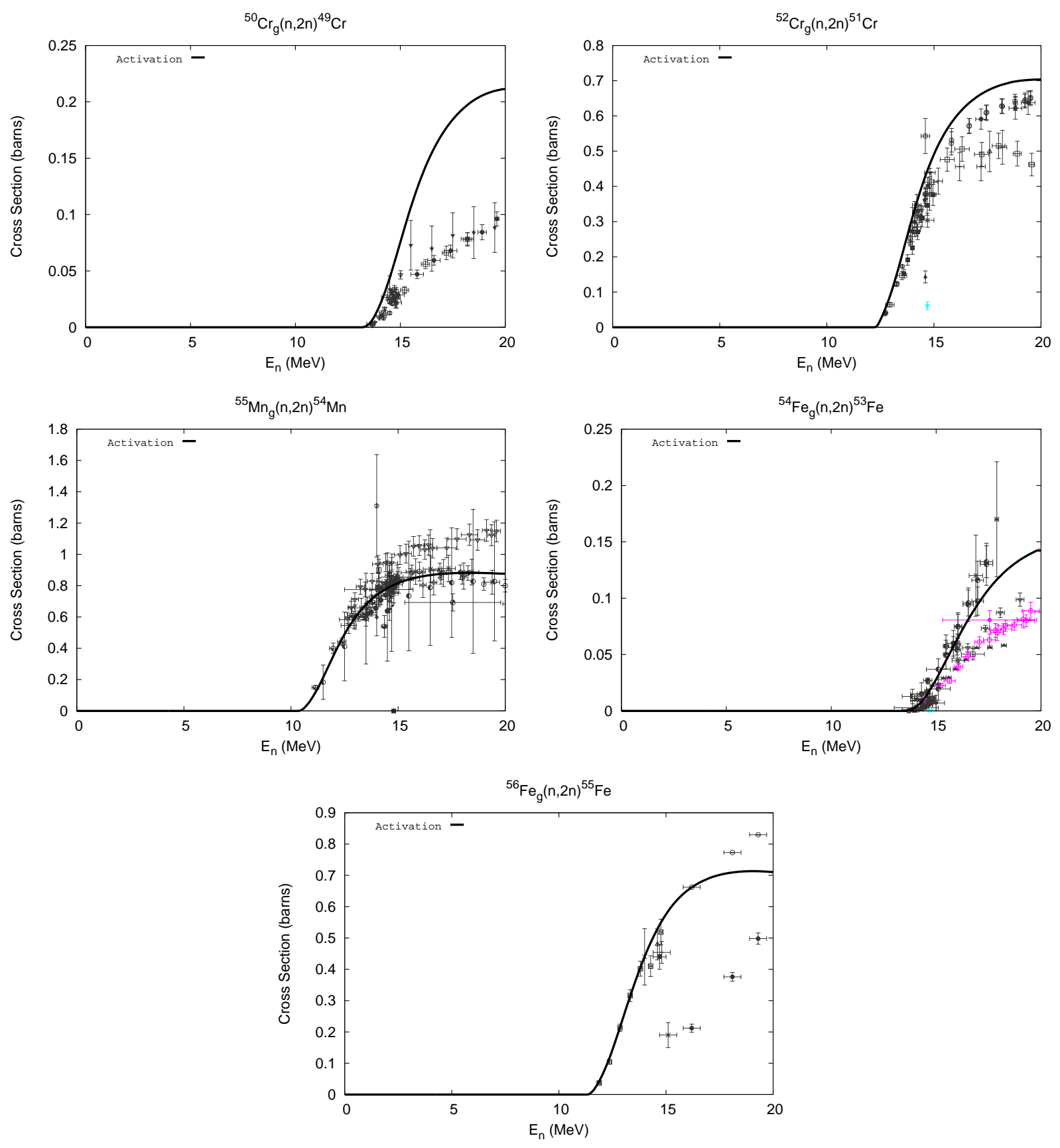

Fig. 24.- (continued) 


\section{B.4. Modeled vs. Measured Cross Sections - (n,p)}
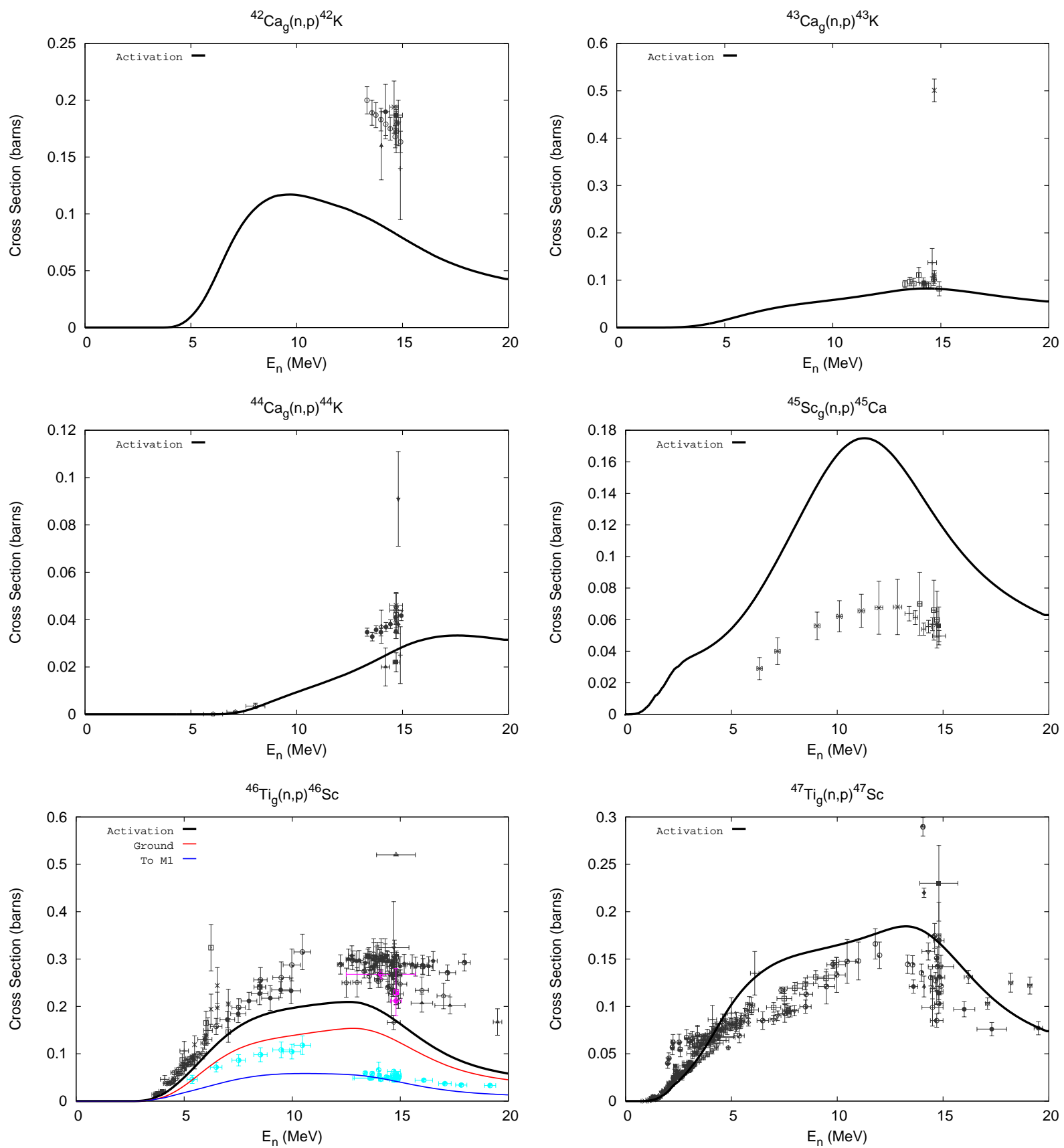

Fig. 25.- Measured vs. calculated cross sections for (n,p) reactions 

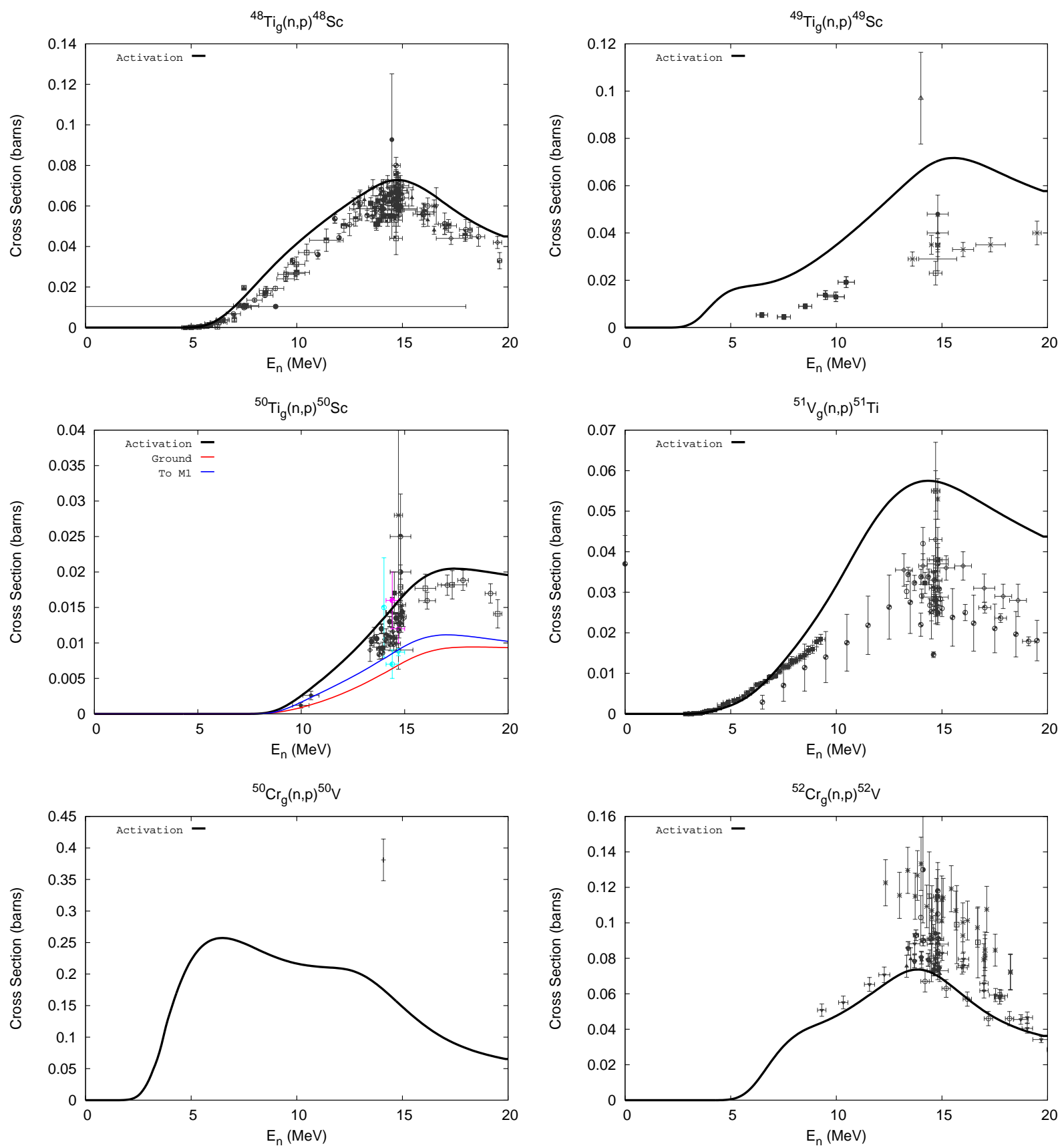

Fig. 25.- (continued) 

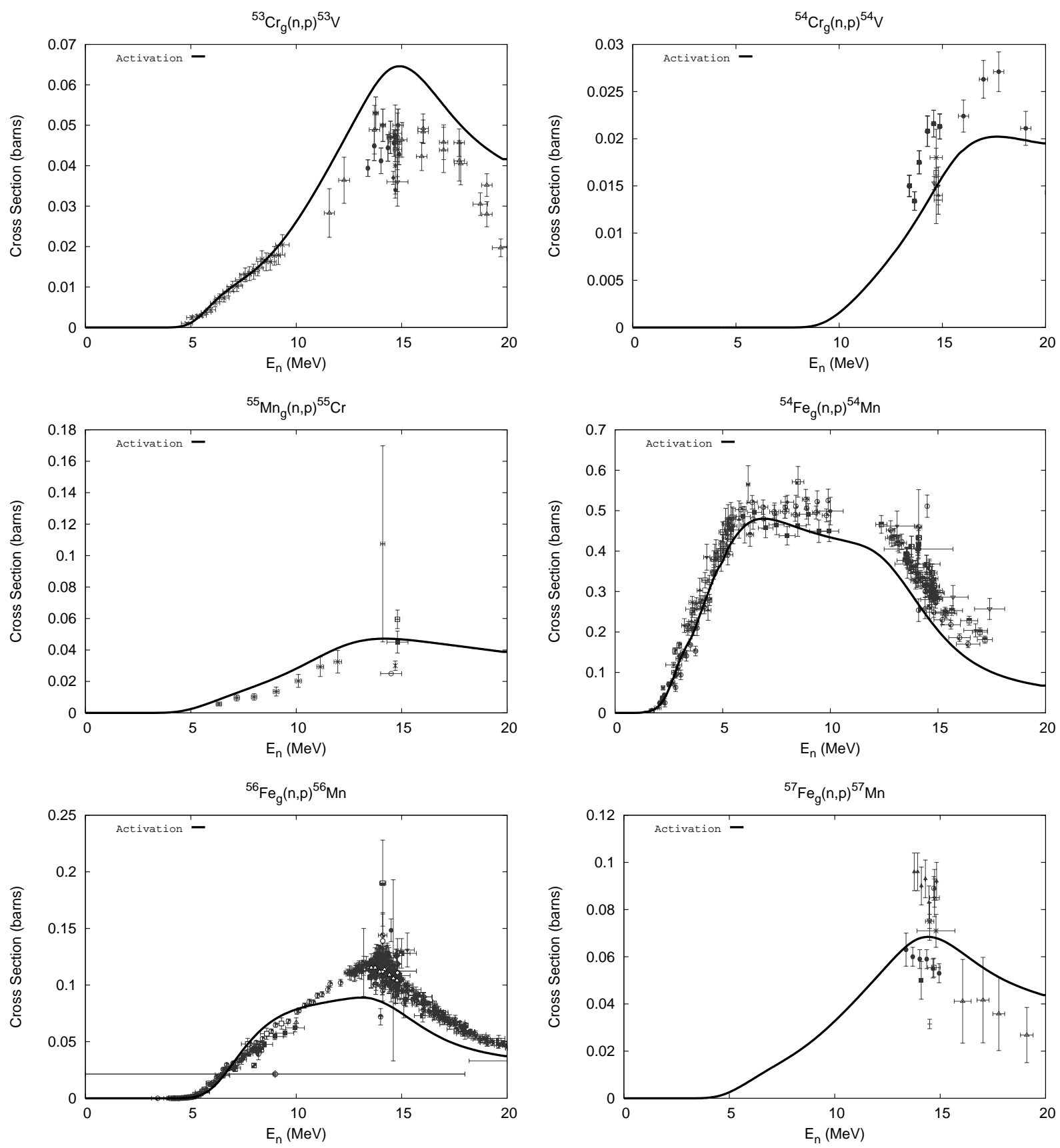

Fig. 25.- (continued) 


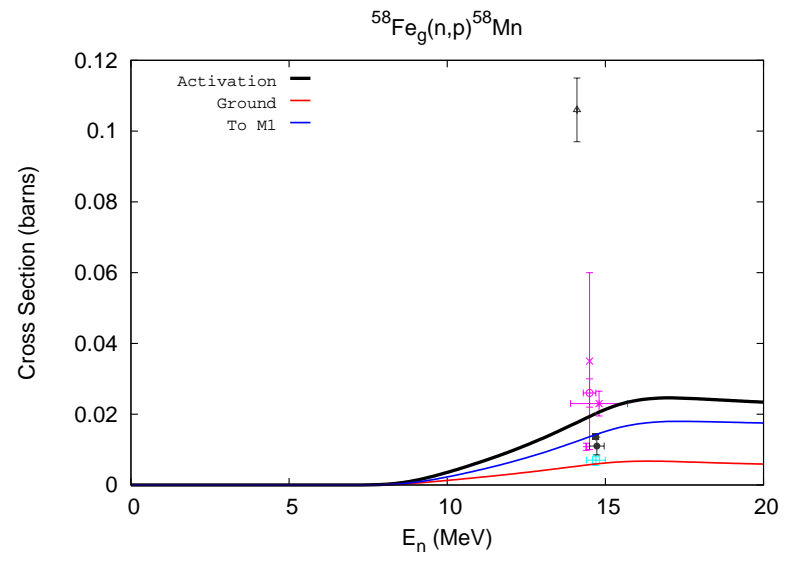

Fig. 25.- (continued) 


\section{B.5. Modeled vs. Measured Cross Sections - $(\mathrm{n}, \mathrm{np})+(\mathrm{n}, \mathrm{pn})$}
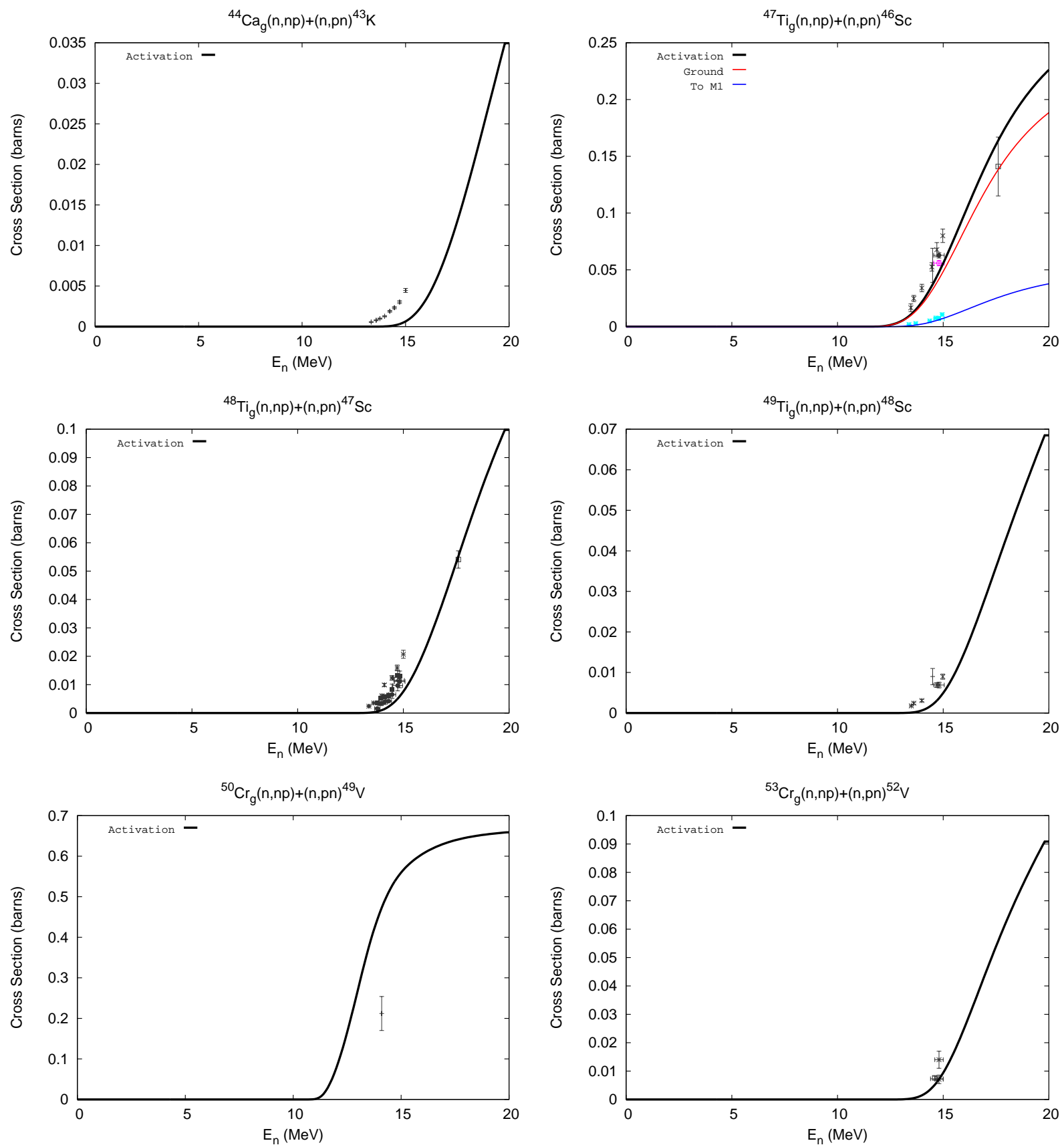

Fig. 26. - Measured vs. calculated cross sections for $(\mathrm{n}, \mathrm{np})+(\mathrm{n}, \mathrm{pn})$ reactions 

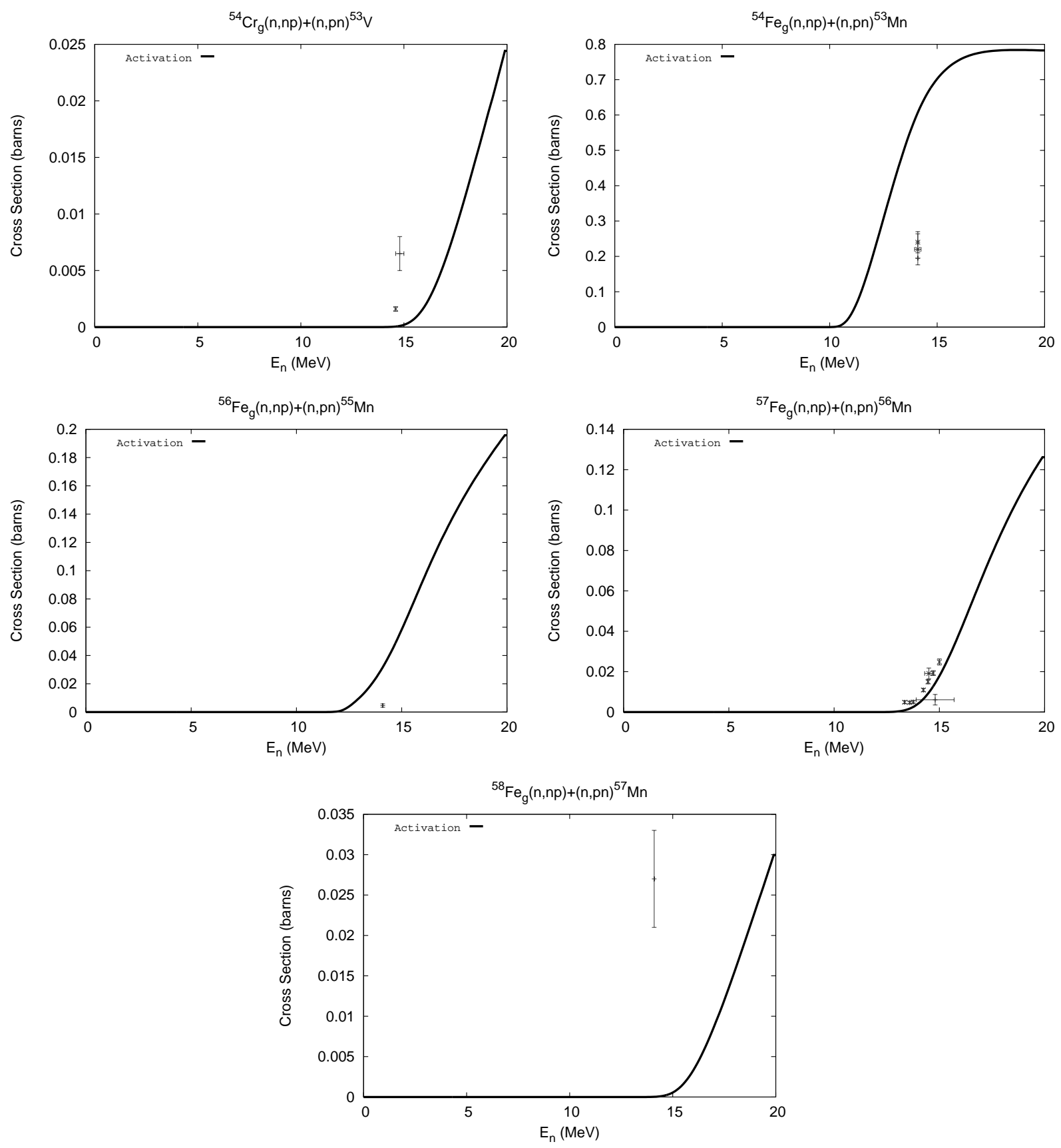

Fig. 26.- (continued) 


\section{B.6. Modeled vs. Measured Cross Sections - $(\mathbf{n}, \alpha)$}
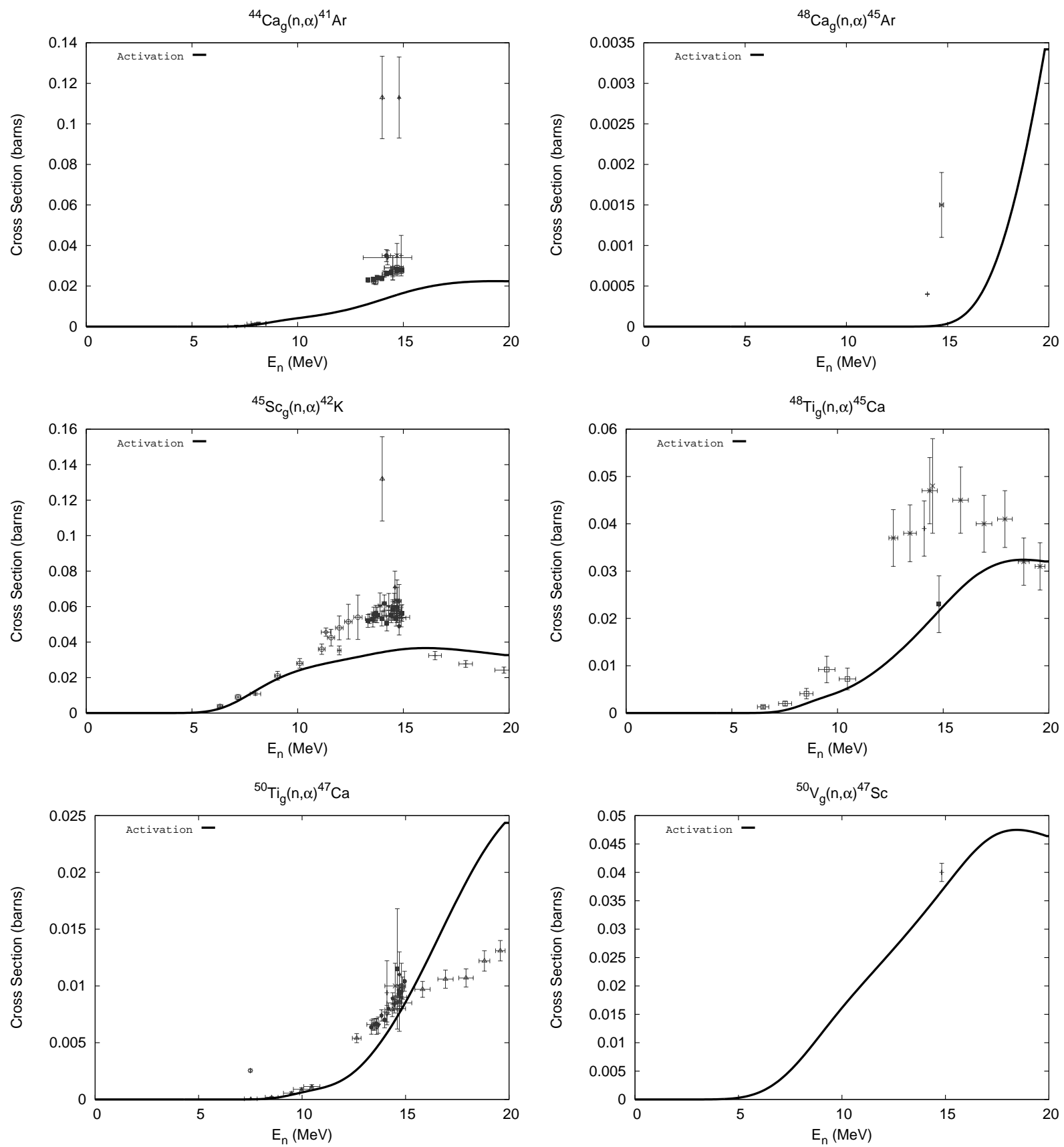

Fig. 27.- Measured vs. calculated cross sections for $(\mathrm{n}, \alpha)$ reactions 

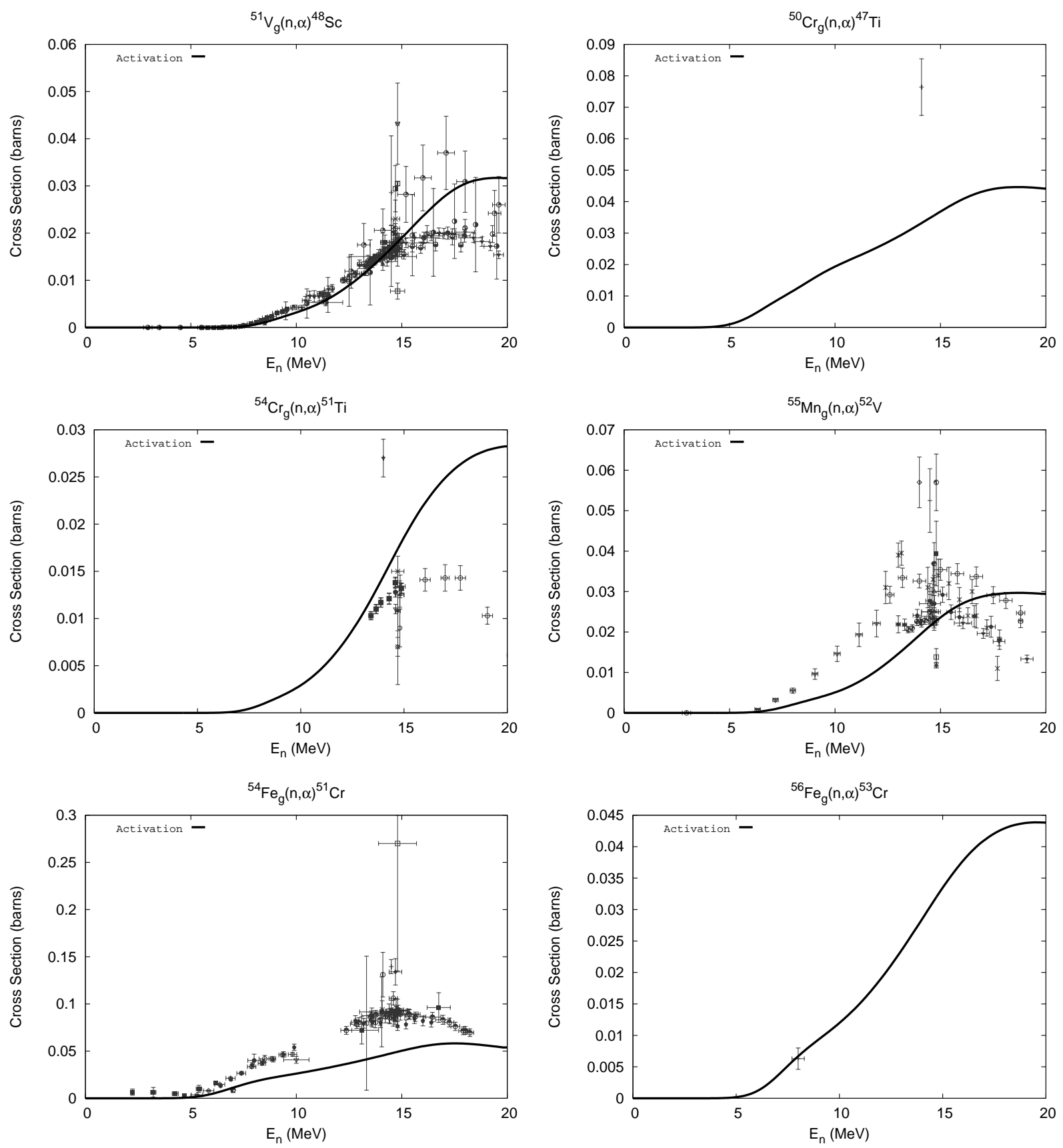

Fig. 27.- (continued) 


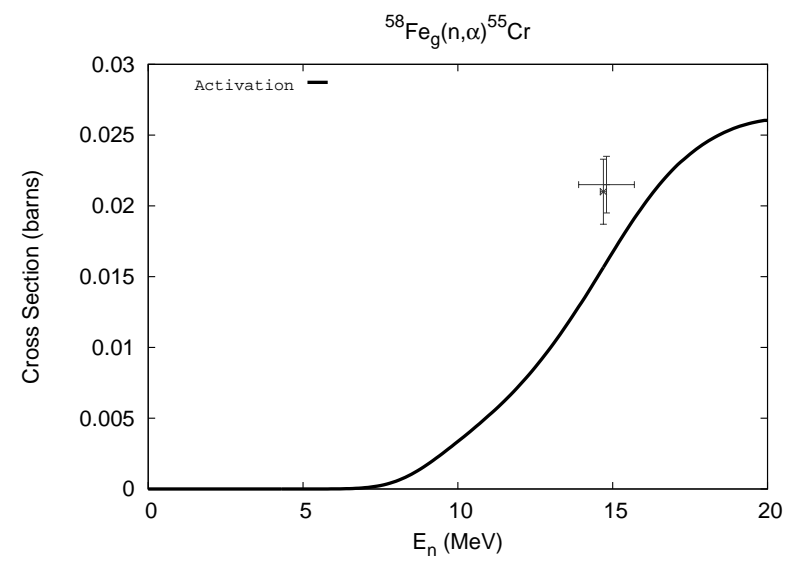

Fig. 27.- (continued) 


\section{B.7. Modeled vs. Measured Cross Sections - (n,d)}
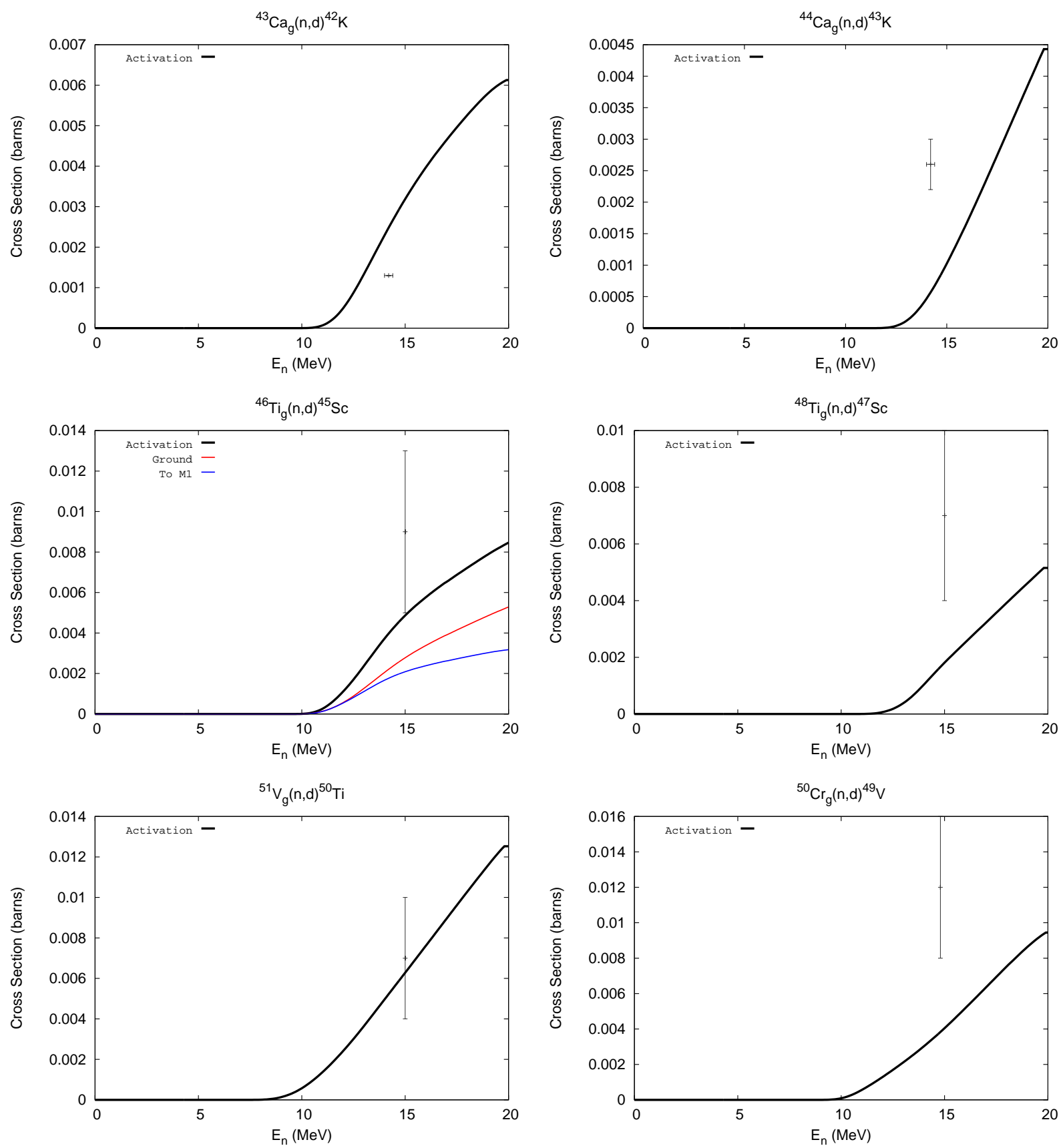

Fig. 28.- Measured vs. calculated cross sections for (n,d) reactions 

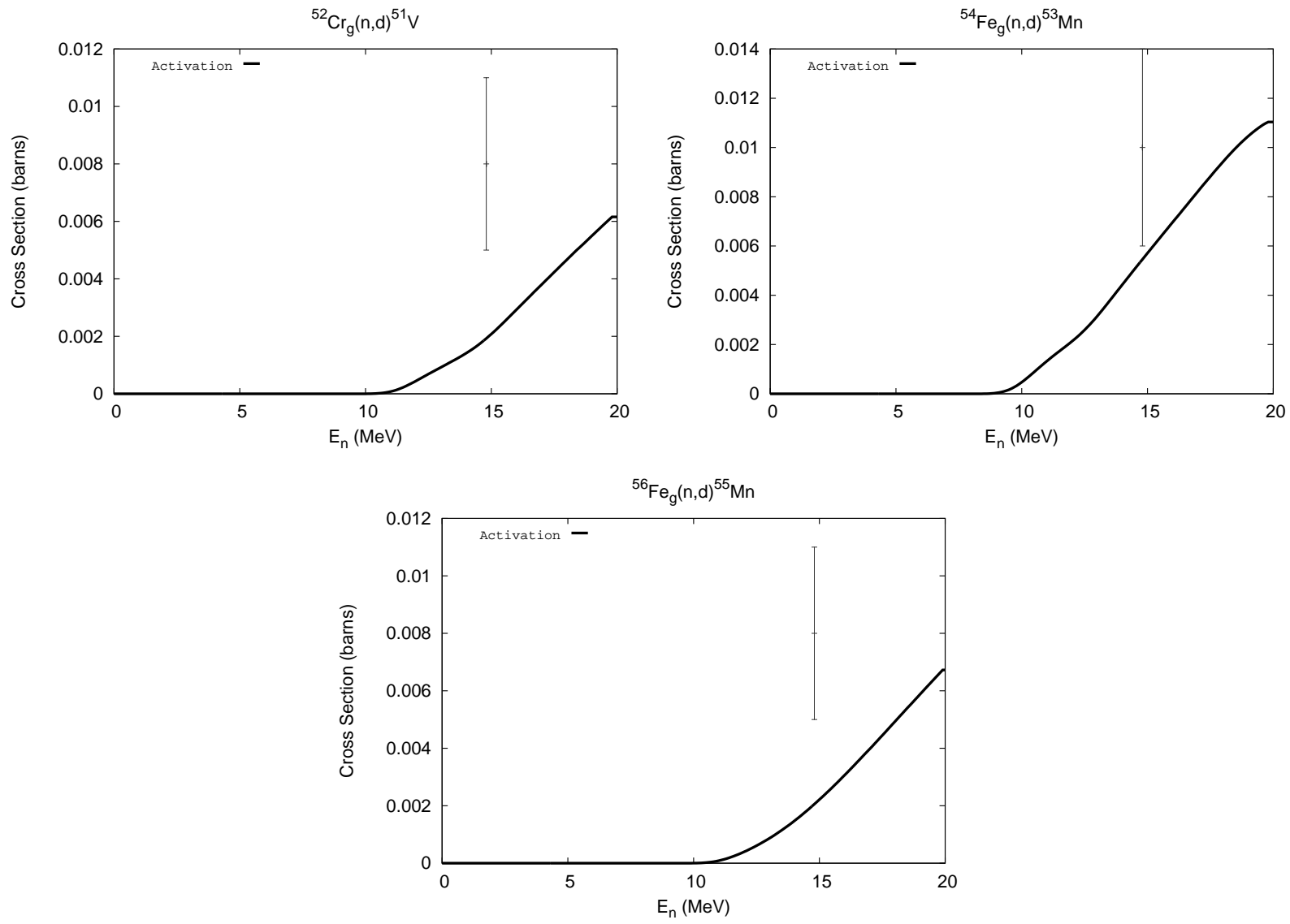

Fig. 28.- (continued) 


\section{B.8. Modeled vs. Measured Cross Sections - (n,n')}
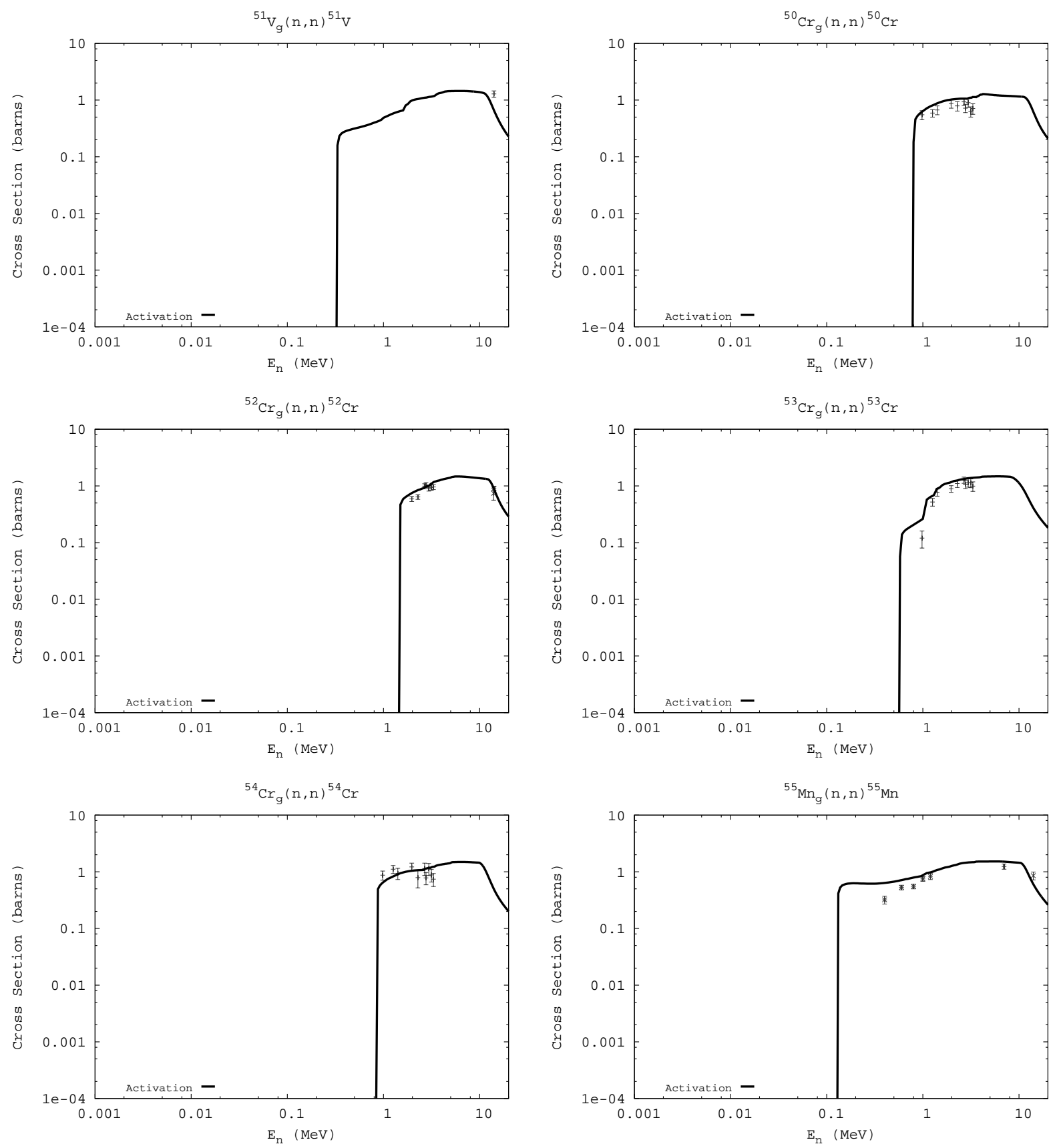

Fig. 29.- Measured vs. calculated cross sections for (n,n') reactions 

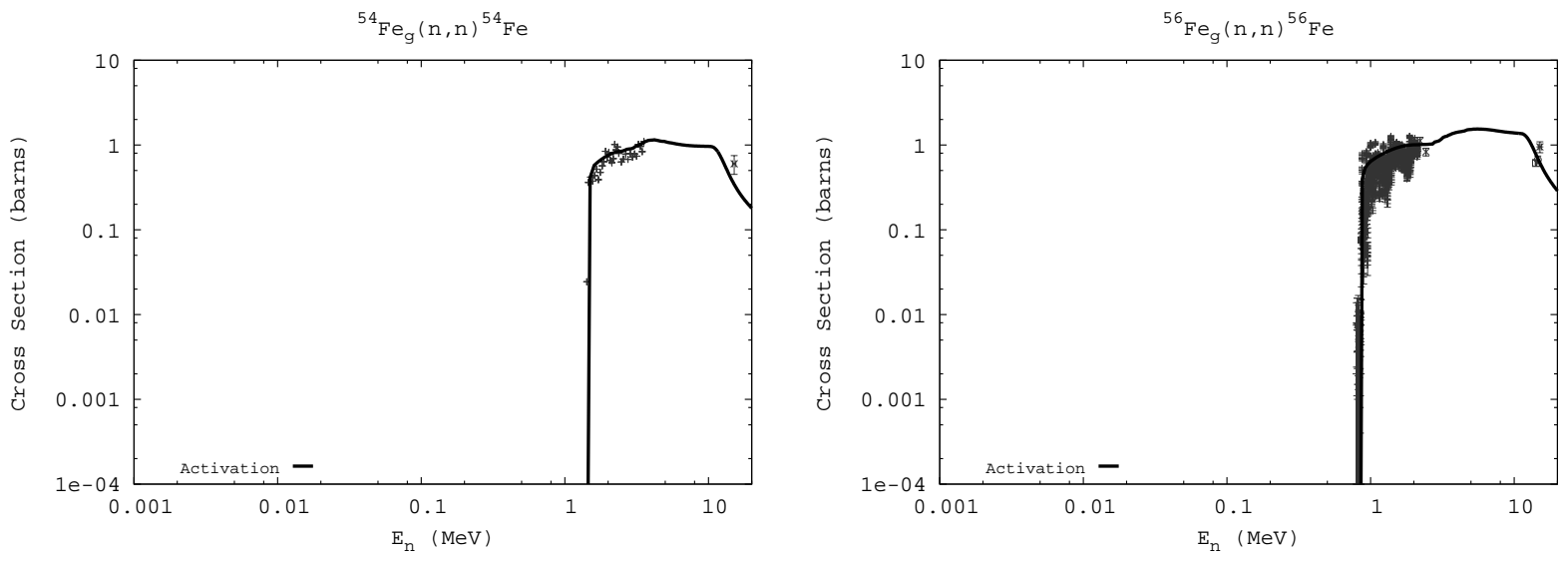

Fig. 29.- (continued) 
B.9. Modeled vs. Measured Cross Sections - (p,n)
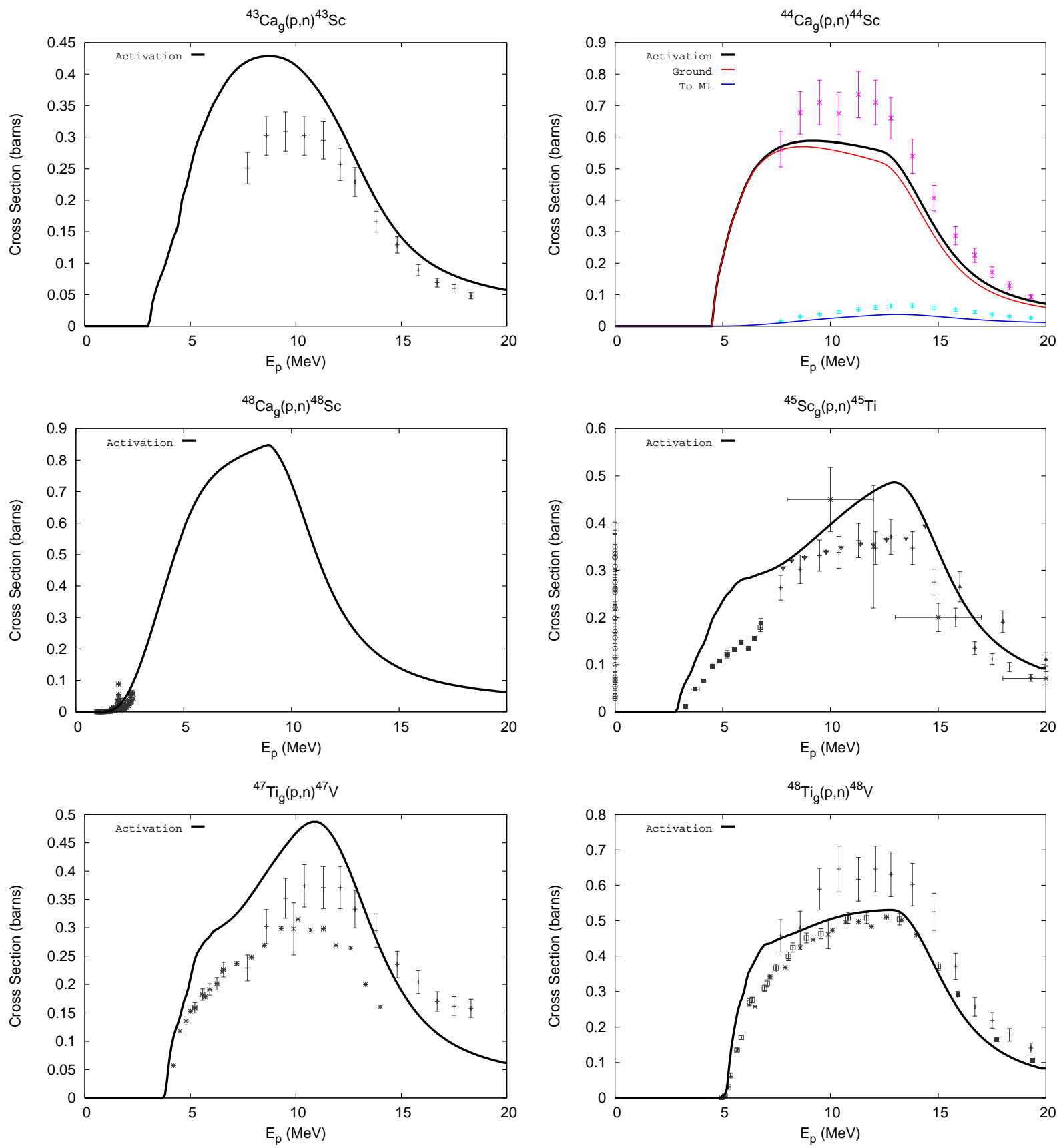

Fig. 30.- Measured vs. calculated cross sections for $(\mathrm{p}, \mathrm{n})$ reactions 

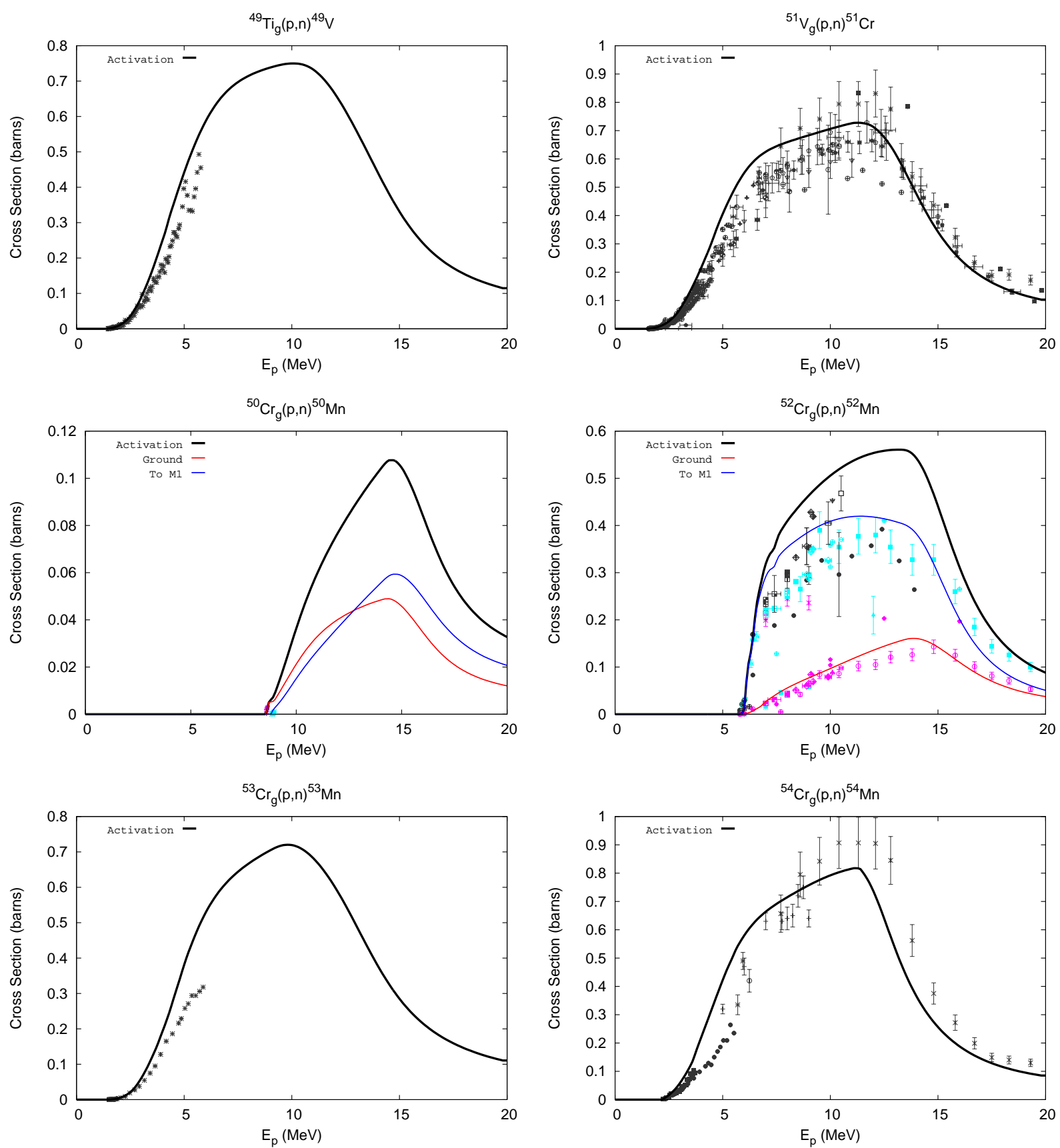

Fig. 30.- (continued) 

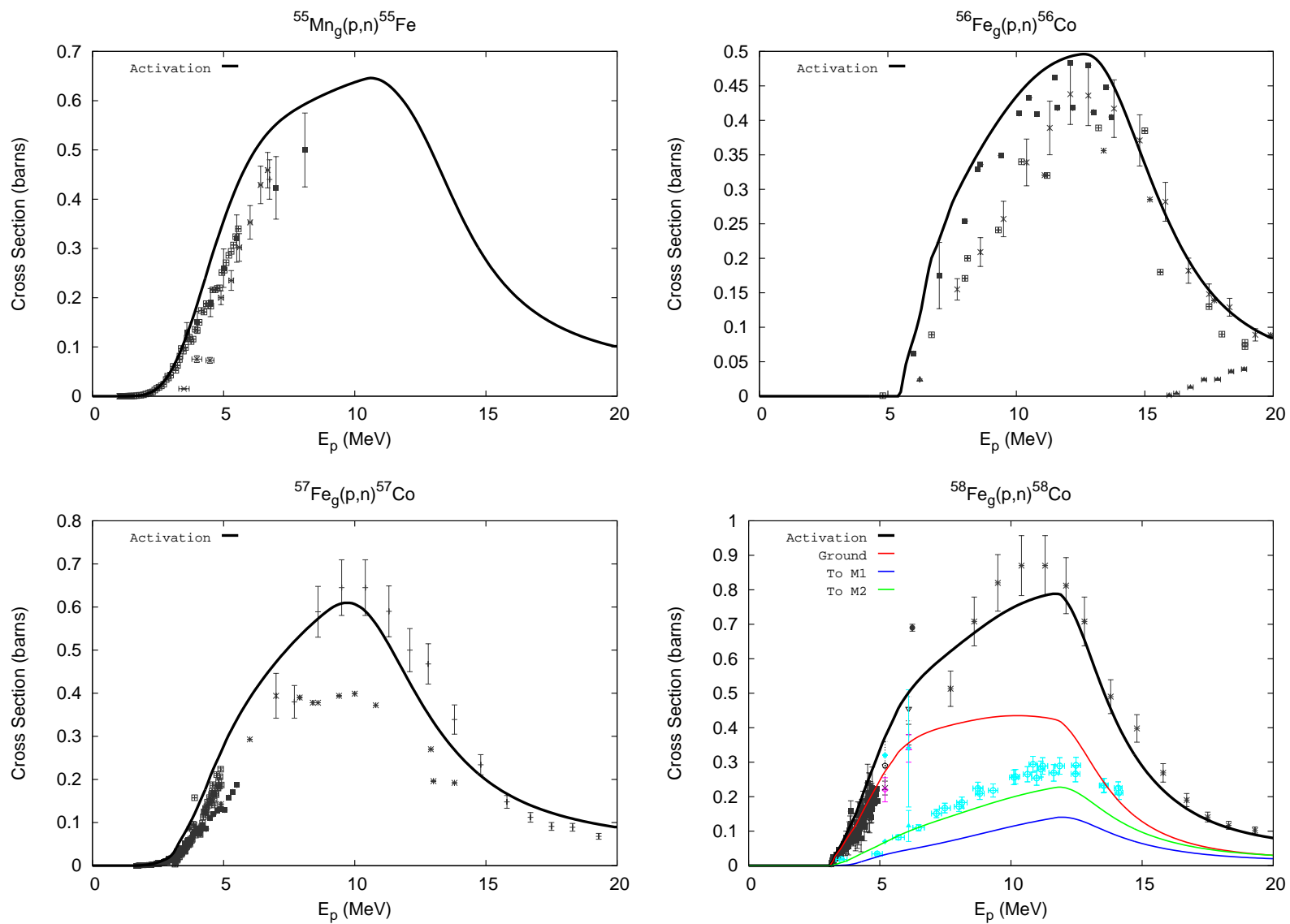

Fig. 31. - Measured vs. calculated cross sections for $(\mathrm{p}, \mathrm{n})$ reactions on ${ }^{58} \mathrm{Fe}$. 
B.10. Modeled vs. Measured Cross Sections - $(\mathbf{p}, 2 \mathrm{n})$
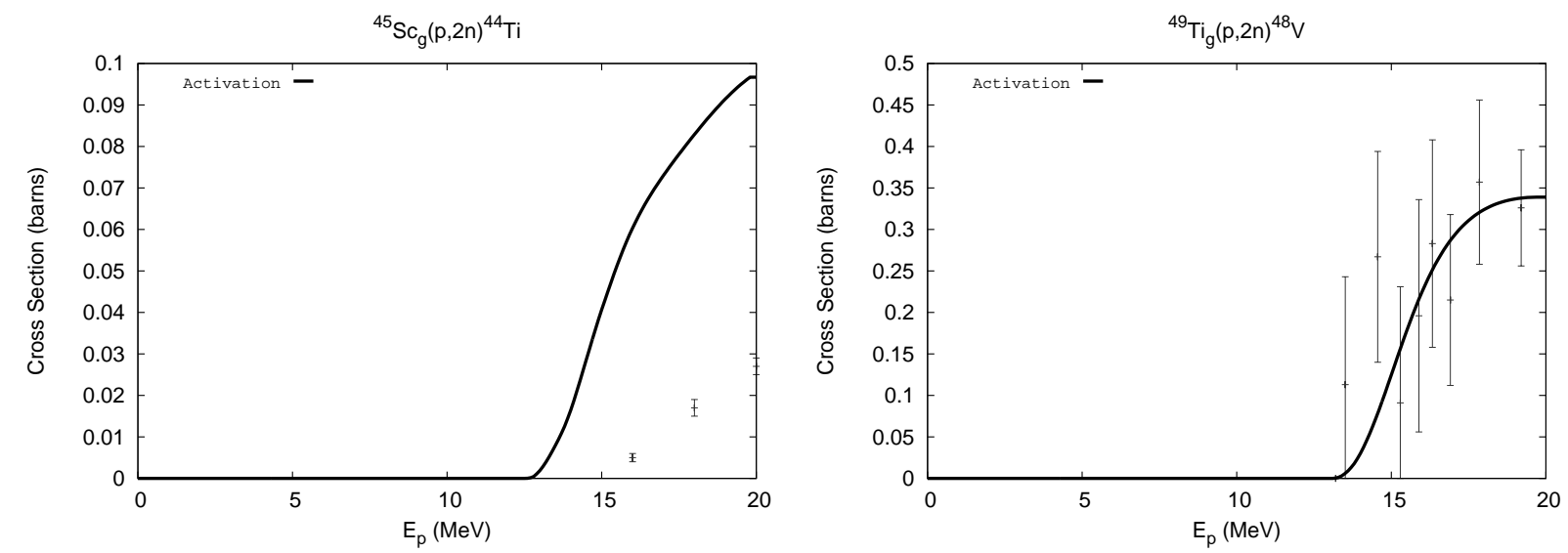

Fig. 32.- Measured vs. calculated cross sections for $(\mathrm{p}, 2 \mathrm{n})$ reactions 
B.11. Modeled vs. Measured Cross Sections - (d,n)
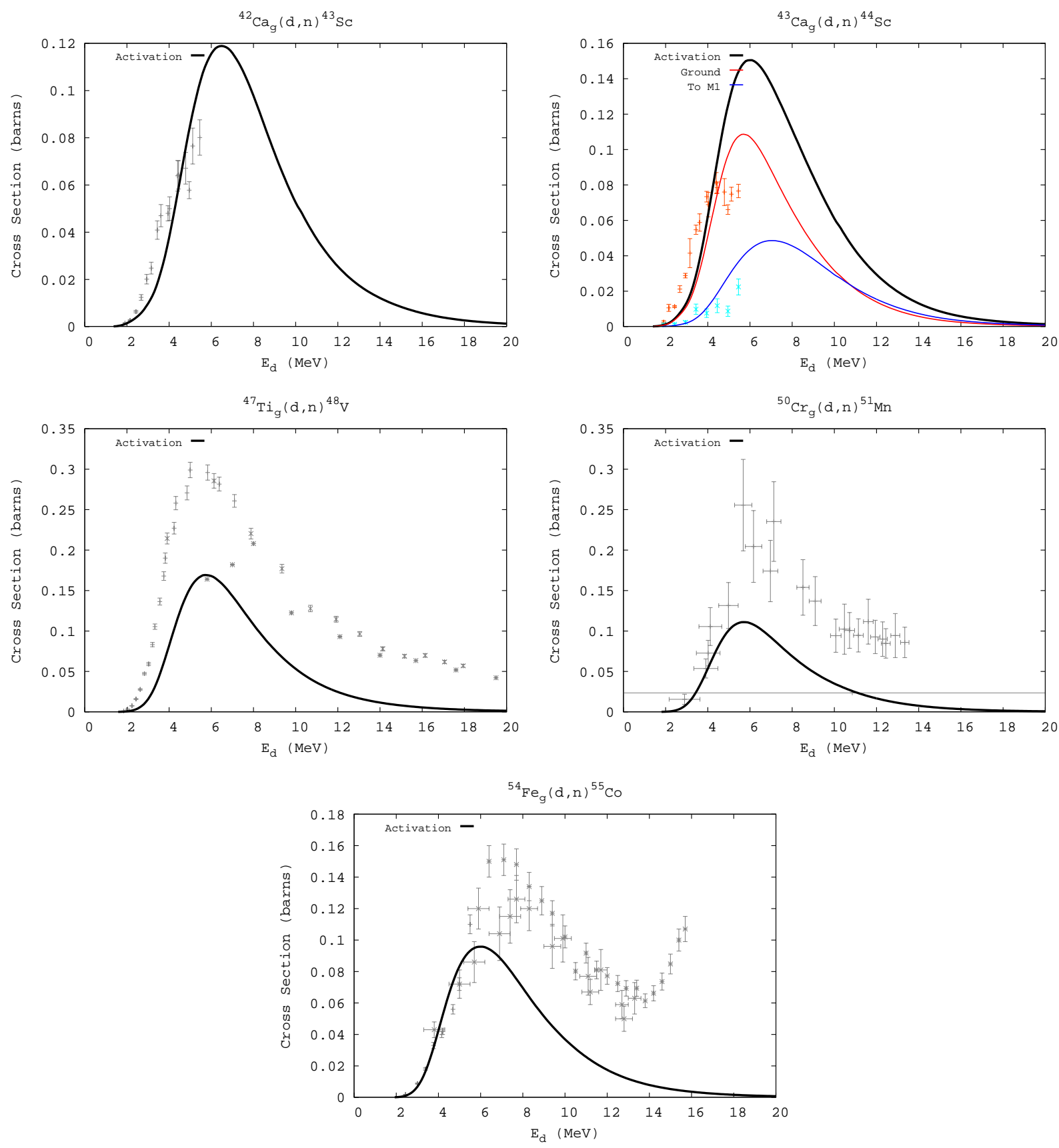

Fig. 33.- Measured vs. calculated cross sections for $(\mathrm{d}, \mathrm{n})$ reactions 


\section{B.12. Modeled vs. Measured Cross Sections - (d,2n)}
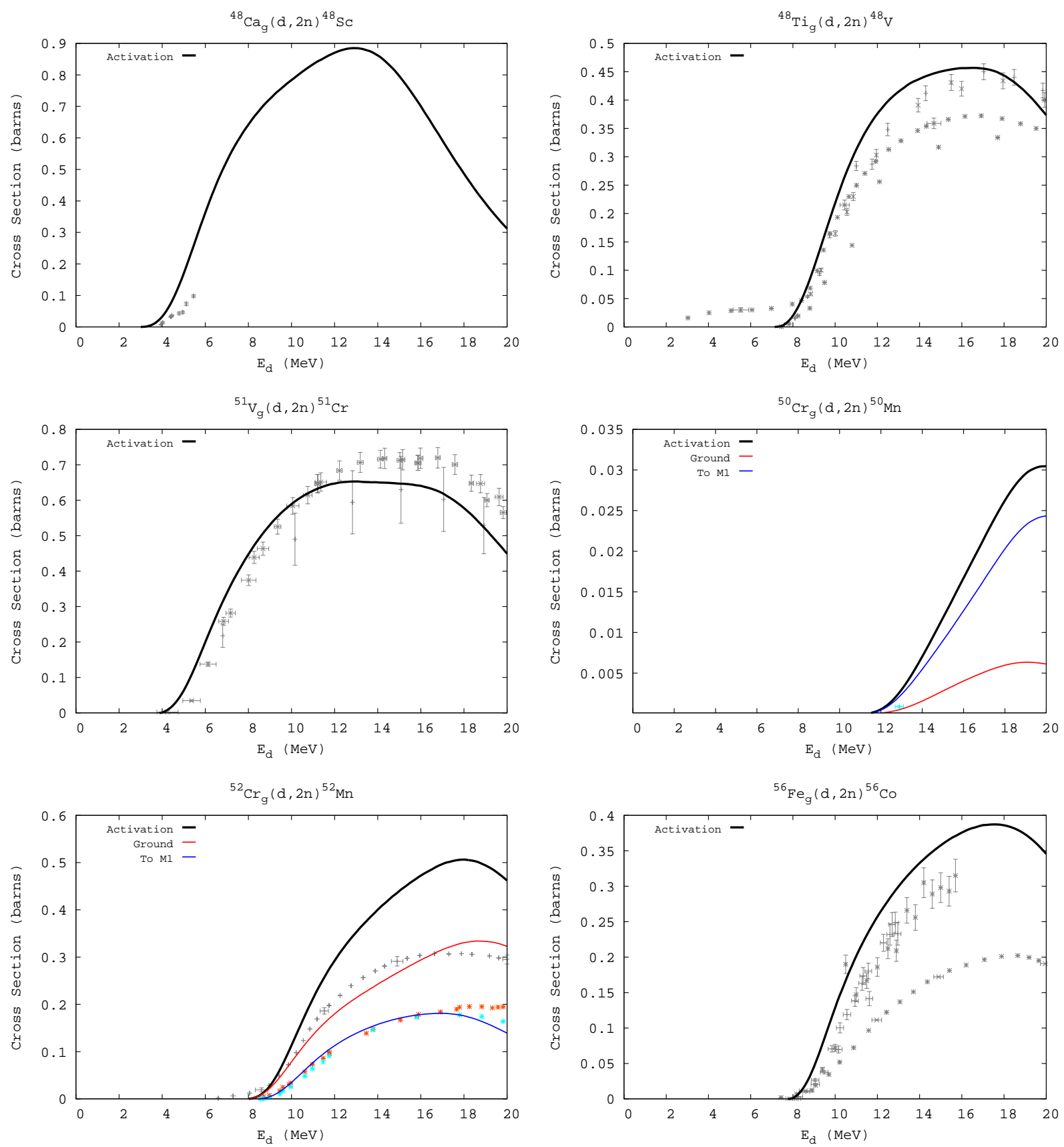

Fig. 34.- Measured vs. calculated cross sections for $(\mathrm{d}, 2 \mathrm{n})$ reactions 


\section{Modeled Cross Sections: Production and Destruction Channels}
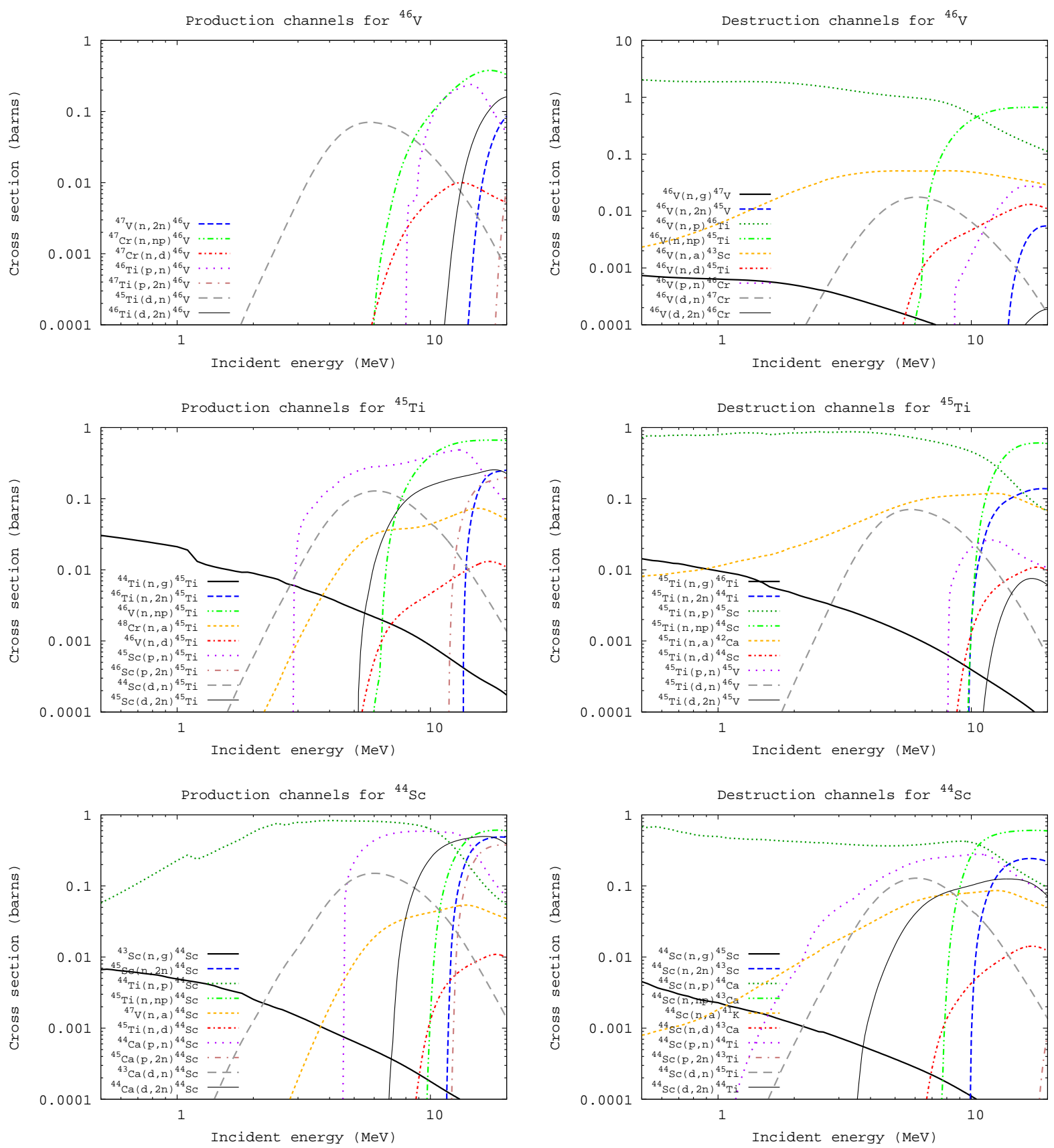

Fig. 35.- Production and destruction cross sections for Sc, Ti, \& V 

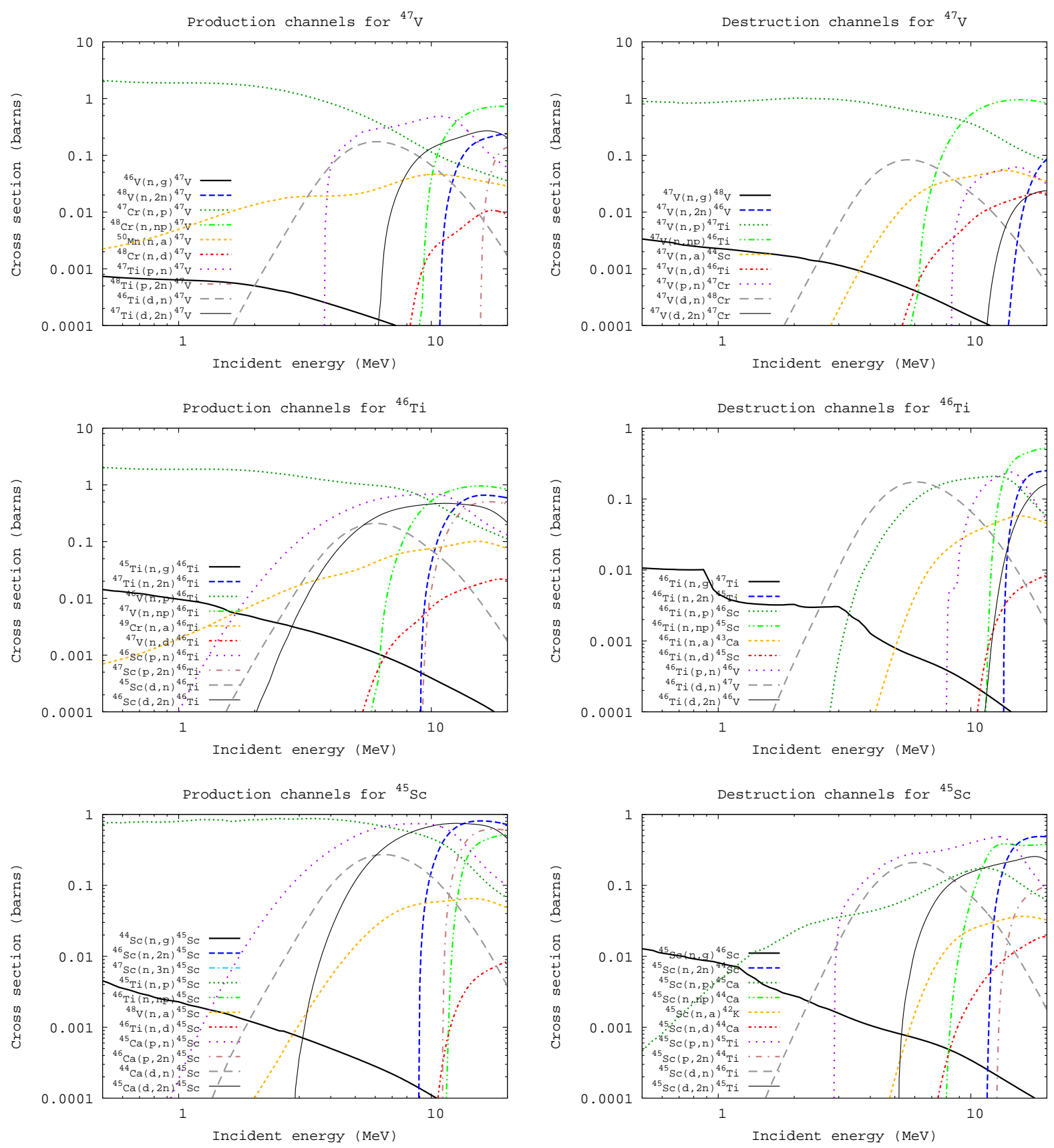

Fig. 35.- (continued) 

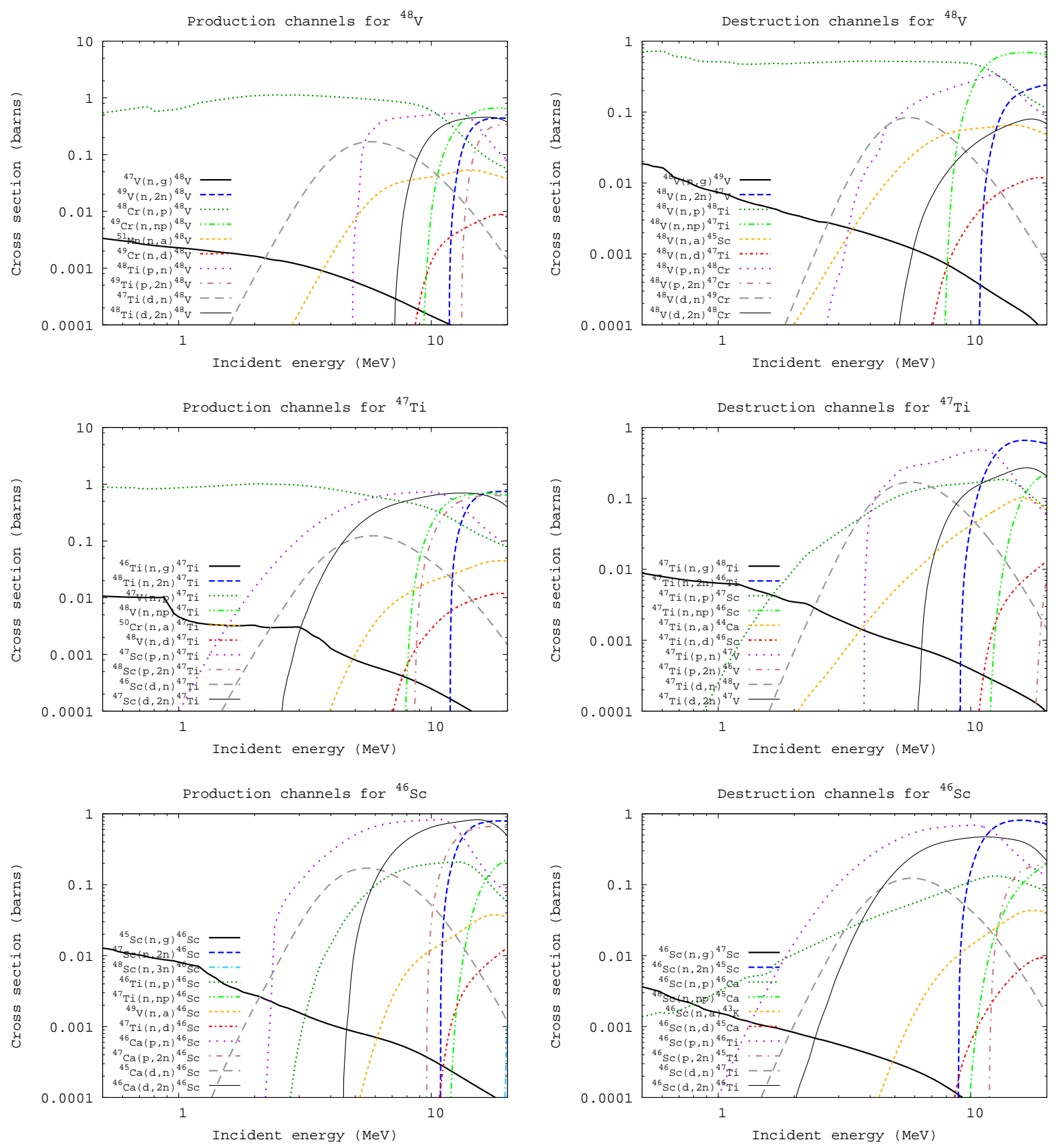

Fig. 35.- (continued) 

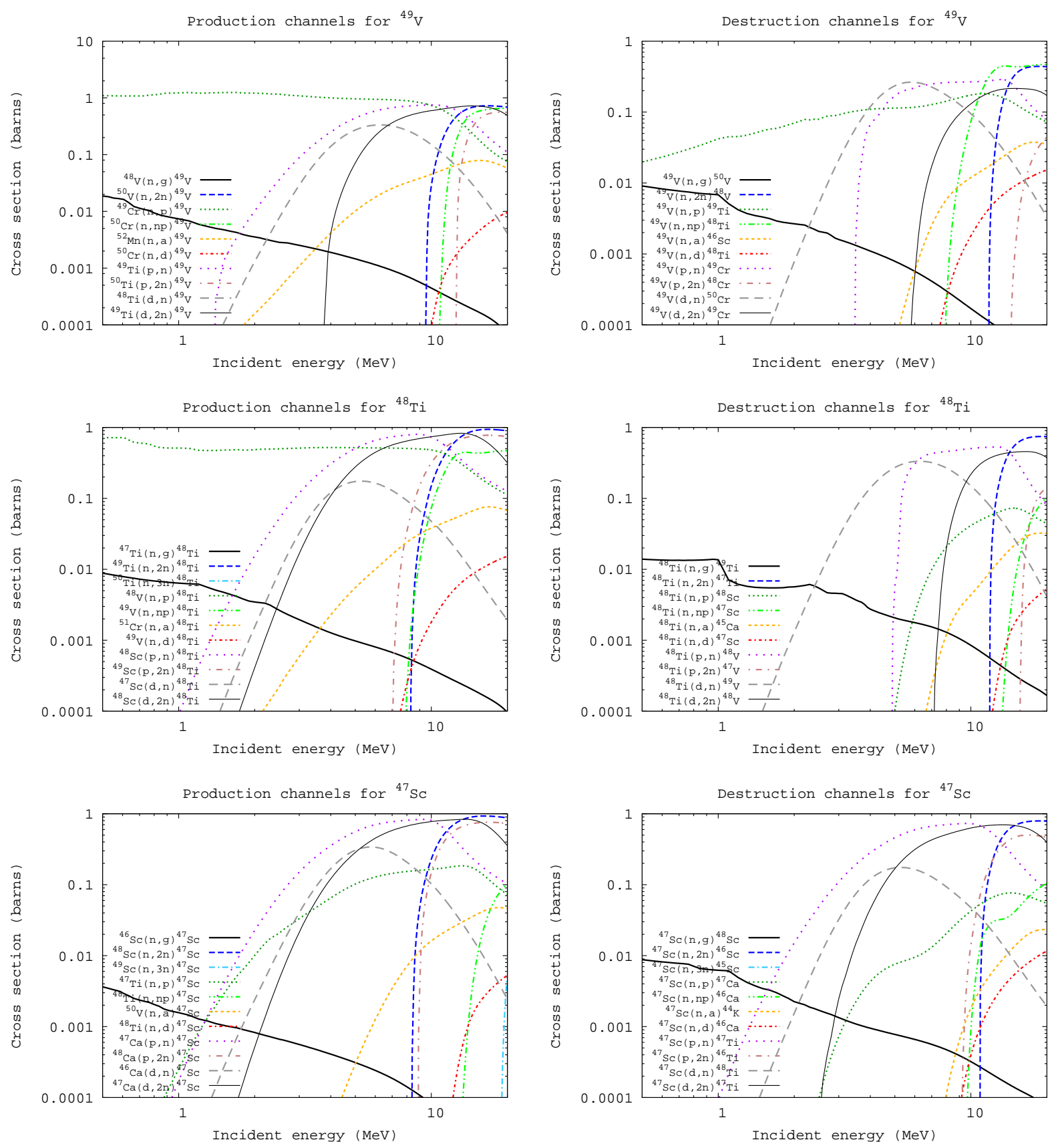

Fig. 35.- (continued) 

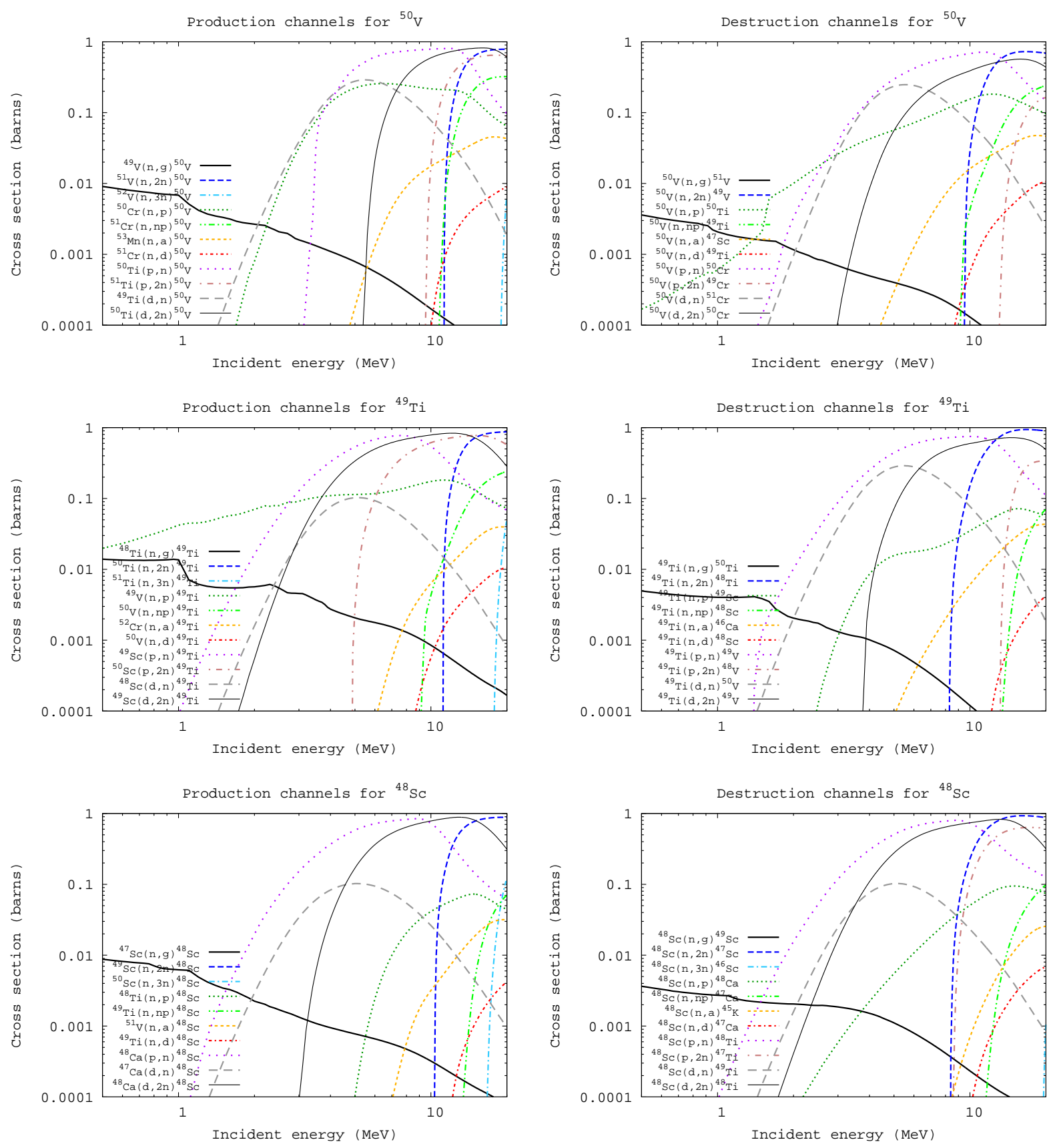

Fig. 35.- (continued) 

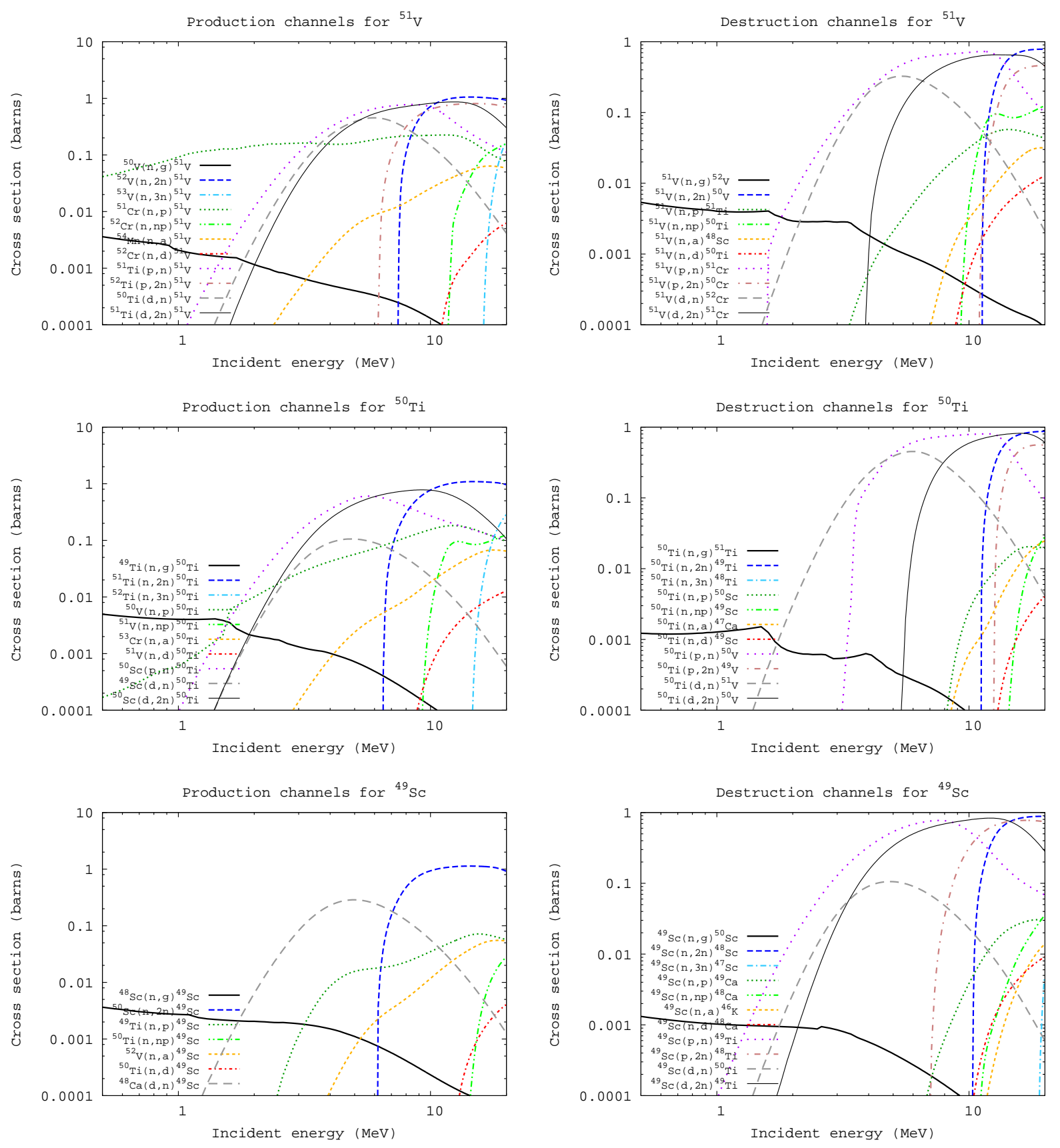

Fig. 35.- (continued) 

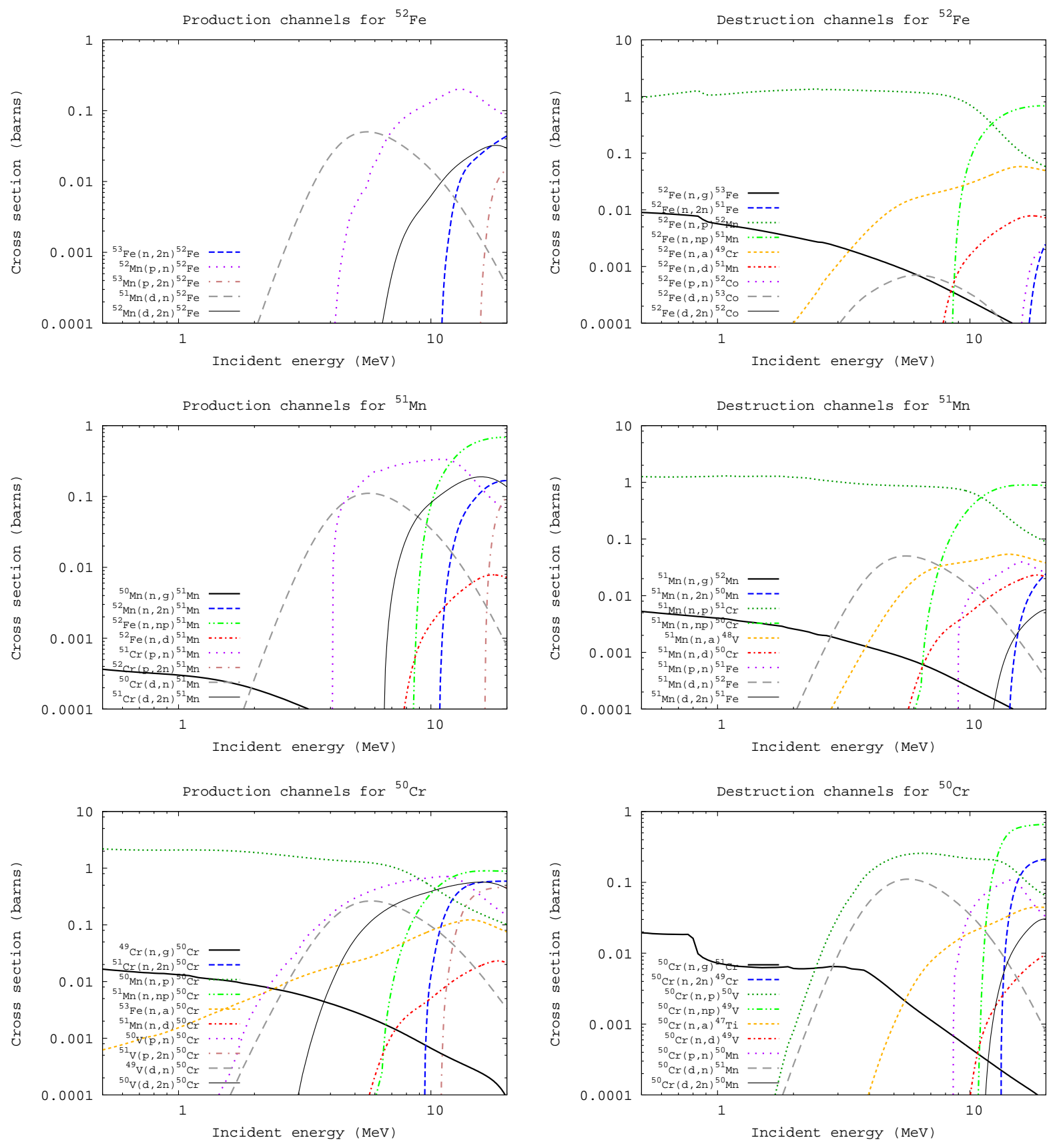

Fig. 36.- Production and destruction cross sections for $\mathrm{Cr}, \mathrm{Mn}, \& \mathrm{Fe}$ 

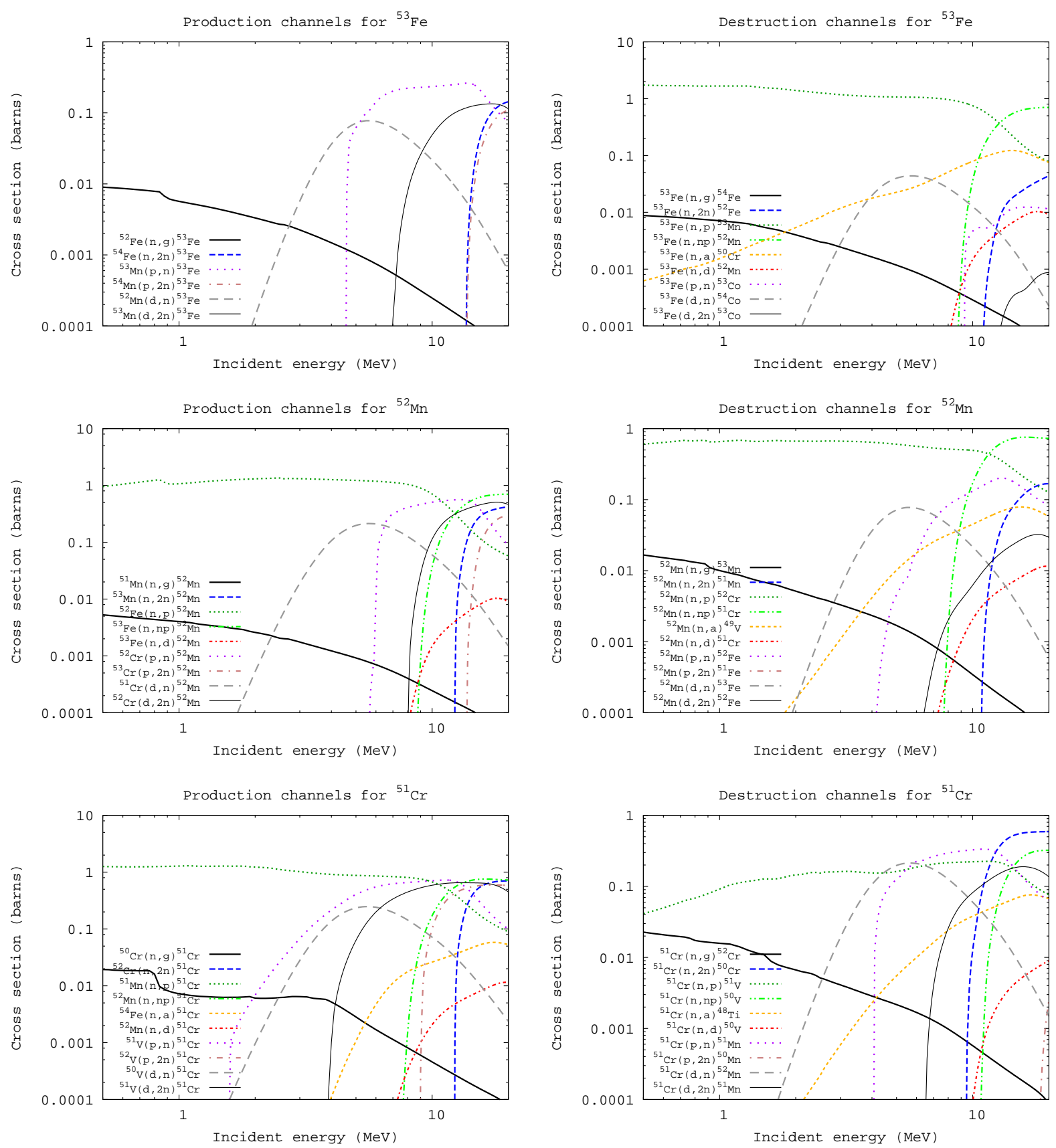

Fig. 36.- (continued) 

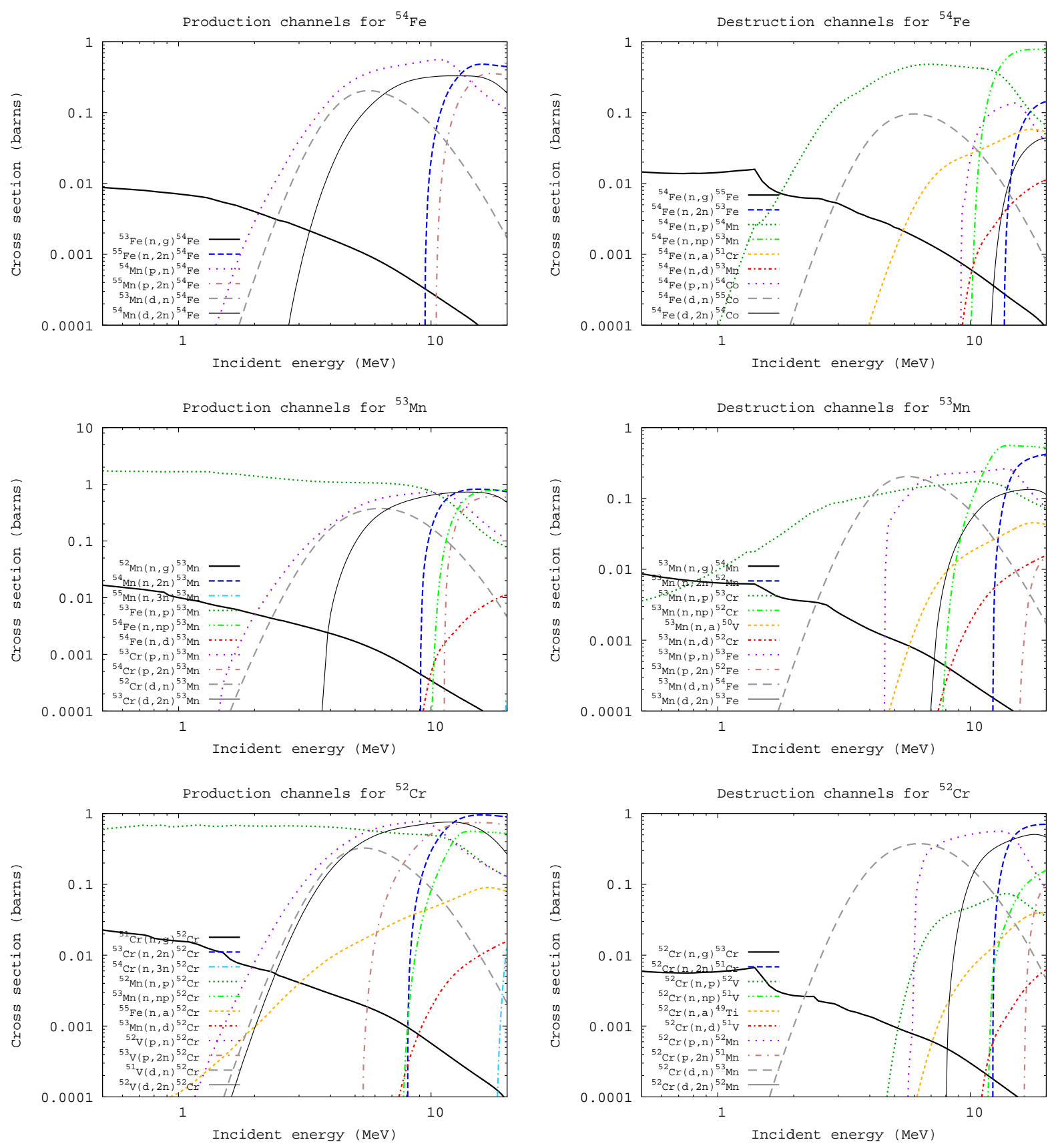

Fig. 36.- (continued) 

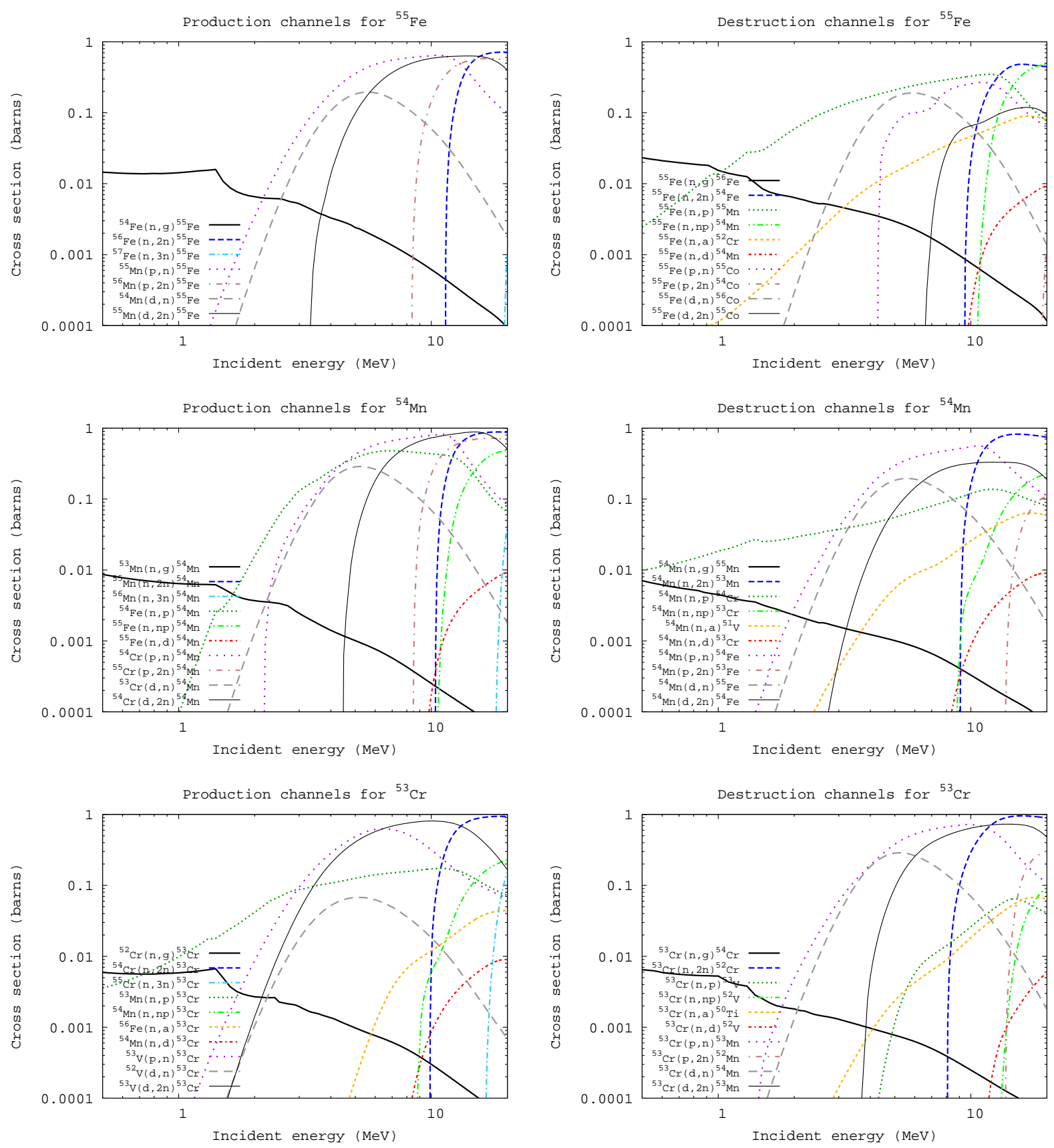

Fig. 36.- (continued) 